\title{
ANALYTICAL MODELING OF PARTICLE INTERFERENCE IN MICRO-ABRASIVE JETS
}

\author{
by \\ Tom Burzynski, \\ Bachelor of Engineering, Mechanical Engineering, \\ Ryerson University, 2005
}

A thesis

presented to Ryerson University

in partial fulfillment of the

requirements for the degree of

Master of Applied Science

in the Program of

Mechanical Engineering

Toronto, Ontario, Canada, 2007

CTom Burzynski 2007

PROPERTY OF 


\section{Author's Declaration}

I hereby declare that I am the sole author of this thesis.

I authorize Ryerson University to lend this thesis to other institutions or individuals for the purpose of scholarly research.

\section{Signature}

I further authorize Ryerson University to reproduce this thesis by photocopying or by other means, in total or in part, at the request of other institutions or individuals for the purpose of scholarly research.

Signature 


\title{
Tom Burzynski
}

Analytical Modeling of Particle Interference in Micro-abrasive Jets

\author{
2007 \\ Master of Applied Science \\ Mechanical Engineering \\ Ryerson University
}

\begin{abstract}
Two existing analytical models of particle interference in non-divergent particle jets were modified to include radial symmetry of the particle jet across the nozzle axis, inter-particle spacing, and more complex rebound geometry. Two novel experimental techniques for obtaining the particle spatial and velocity distributions across a micro-abrasive jet were then devised and rigorously tested. One of the above mentioned analytical models was then chosen to be further modified to include the above-mentioned modifications along with the effect of a divergent jet, the ability to simulate high flux cases, and the experimentally obtained particle spatial and velocity distributions. All of the models were tested at various jet conditions. The results of the models were quantitatively compared to a previously developed computer simulation and were found to qualitatively agree with previous experimental observations. The modified models allow the critical flux below which inter-particle interference is likely to occur to be determined.
\end{abstract}




\section{Acknowledgements}

The author of this work would like to thank Dr. M. Papini for continual guidance and support in the research related to and the writing of this thesis.

I am very thankful to my parents and my girlfriend, Magda, for their continual moral and emotional support.

The author gratefully acknowledges the financial support of the Natural Sciences and Engineering Research Council of Canada (NSERC). 


\section{Table of Contents}

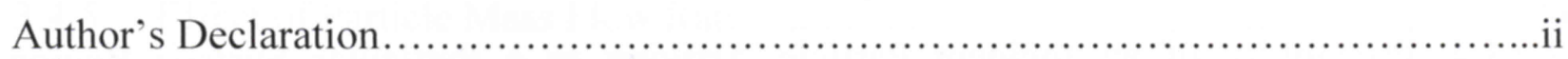

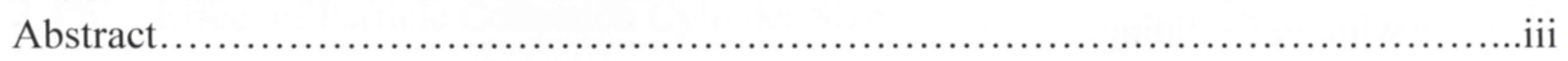

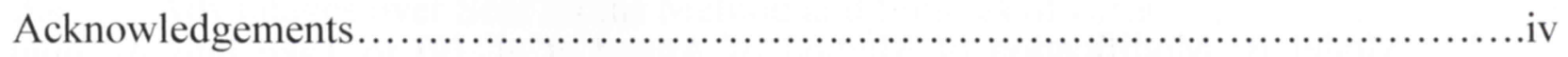

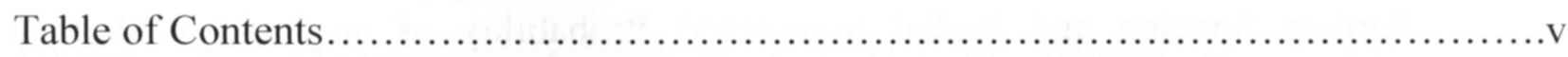

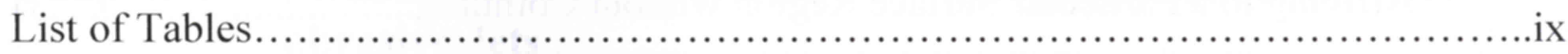

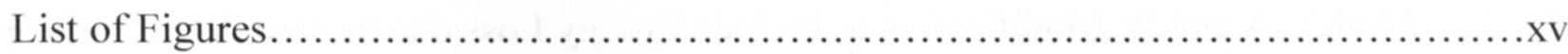

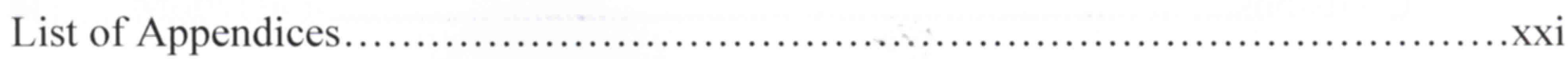

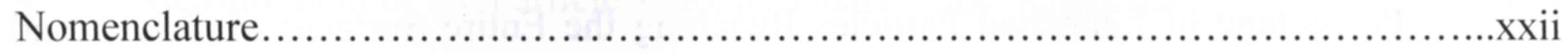

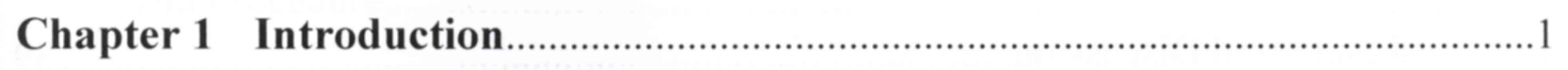

$1.1 \quad$ Background and Critical Literature Review.......................................

1.1.1 Particle Interference Effects in Erosion Testing..................................

1.1.2 Analytical Modeling of Particle Interference..........................................................

1.1.3 Computer Modeling of Particle Interference................................5

1.1.4 Study and Modeling of Spatial and Velocity Distribution in Micro-abrasive

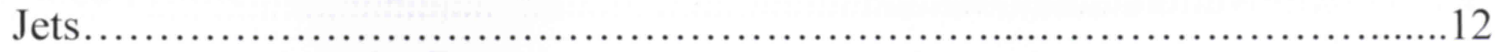

1.1.5 Development of Profile Shape and its Application to Modeling Velocity Distribution in Micro-abrasive Jets............................................14

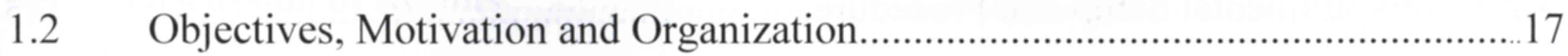

Chapter 2 Analytical Models of the Interference Effects in a Non-divergent Jet at Low Flux Conditions............................................................18

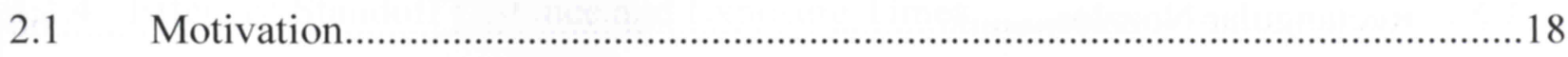

2.2 Modification of Existing Analytical Models of Particle Interference...................220

2.2.1 Model A: Modification of Method of Andrews and Horsfield [8].........................2

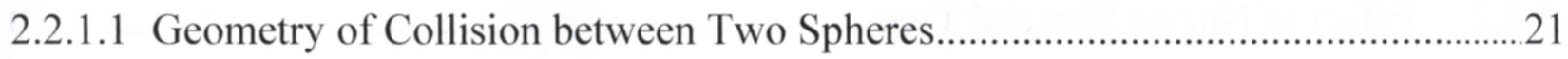

2.2.1.2 Modification to Take into Account Particle Spacing and Radial Symmetry........23 
2.2.1.3 Probability of Collision between Incident and Rebounding Particles at a Given Location in the Incident Jet.

2.2.1.4 Probability of an Incident Particle Arriving to a Particular Surface Region without Colliding.

2.2.2 Model B: Modification of Method of Anand et al. [9] to Take into Account Particle Spacing and Radial Symmetry: Probability of an Incident Particle Arriving to a Particular Surface Region without Colliding. .31

2.2.3 Models A and B: Modification to Include Energy Loss due to Particle-to-Surface Collisions.

2.3 Percentage of Launched Particles Reaching the Entire Surface: Models A and $\mathrm{B}$

2.4 Results and Discussion

2.4.1 Comparison with Computer Simulation of Refs. [10,12].

2.4.2 Qualitative Comparison with Experimental Observations

Chapter 3 Measurement of the Particle Spatial Distribution in Microabrasive Jets. .44

3.1 Motivation. 44

3.2 Measurement of Particle Spatial Distribution across the Jet. 45

3.2.1 Experimental Setup and Procedure .45

3.2.2 Analysis

3.3 Results .53

3.3.1 Round Nozzles. .53

3.3.2 Rectangular Nozzles.. 60

3.4 Discussion of Results. 67

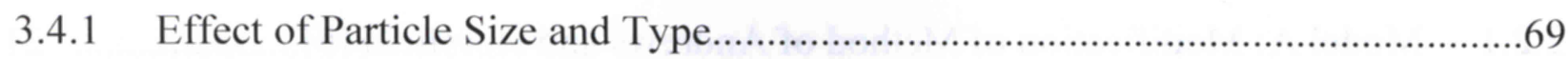

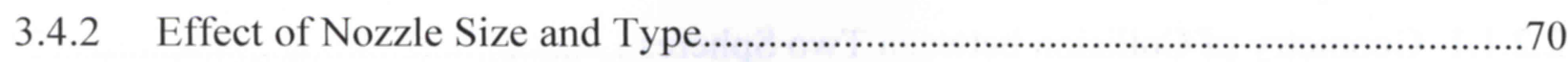

3.4.2.1 Effect of Nozzle Size (Round Nozzles) ............................................................

3.4.2.2 Effect of Nozzle Type (Rectangular Nozzles).................................................... 


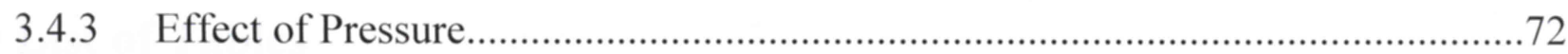

3.4.4 Effect of Standoff Distance and Exposure Time.................................................... 73

3.4.5 Effect of Particle Mass Flow Rate …............................................................................

3.4.6 Effect of Particle Collection Cylinder Size...............................................................

3.4.7 Advantages over Scar radius Method and Sources of Error...................................75

Chapter 4 Measurement of the Particle Velocity Distribution in Micro-

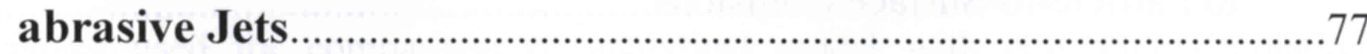

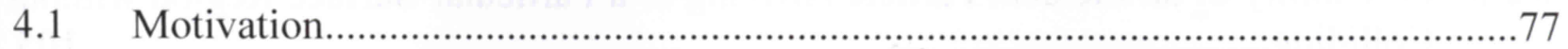

4.2 Measurement of the Particle Velocity Distribution across the Jet..........................78

4.2.1 Erosion Rate Experiments to Obtain the Velocity Exponent: Experimental Setup

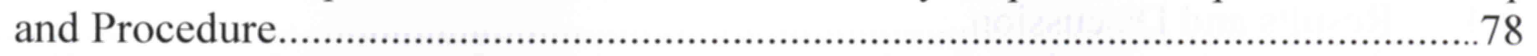

4.2.2 Shallow Profile Experiments: Experimental Setup and Procedure..........................78

4.2.3 Analysis: Relationship between Erosion Rate and Velocity Exponent...................80

4.2.4 Analysis: Relationship between Shallow Eroded Profile, Velocity and Flux..........80

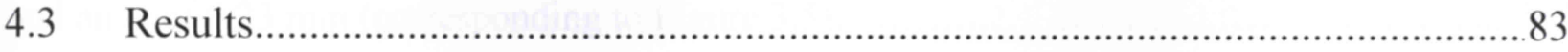

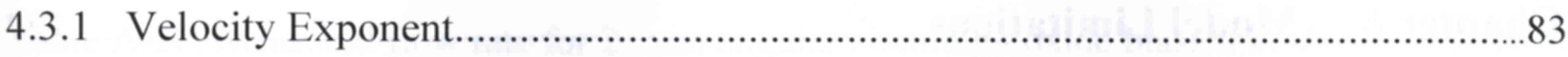

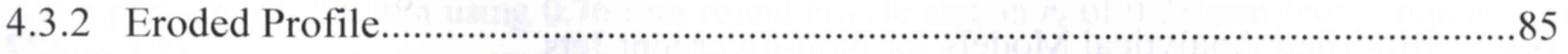

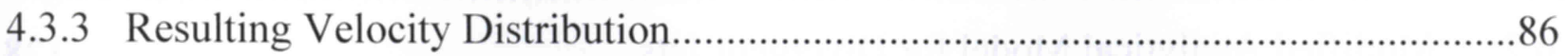

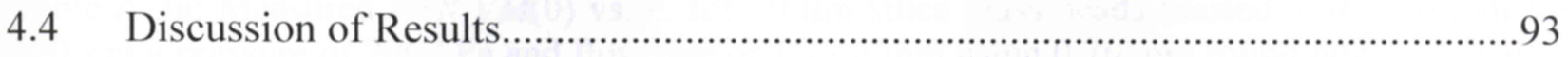

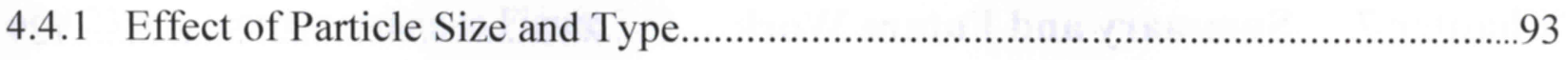

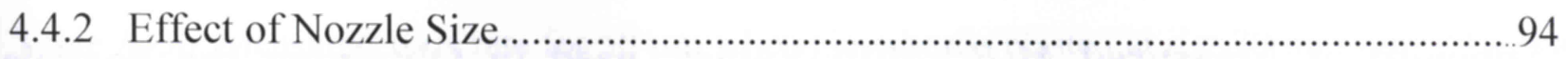

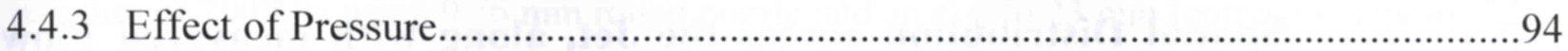

4.4.4 Effect of Standoff Distance and Exposure Times....................................................95

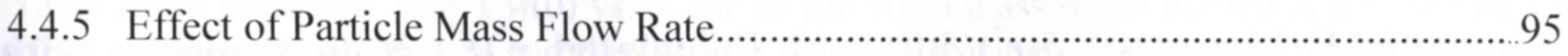

4.4.6 Advantages over Other Methods and Sources of Error...........................................96

Chapter 5 Analytical Model of the Interference Effects in a Divergent Jet

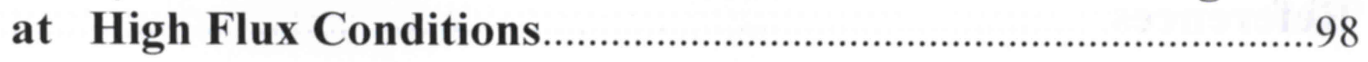




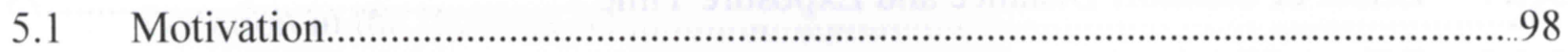

5.2 Modification of Method of Anand et al. [9] ..........................................................99

5.2.1 Modifications to Take into Account Jet Divergence and Particle Spatial

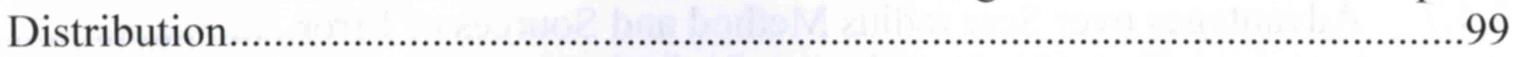

5.2.2 Modification to Include Particle Radial Velocity Distribution and Energy Loss due to Particle-to-Surface Collisions.

5.2.3 Probability of an Incident Particle Arriving to a Particular Surface Region without Colliding...... 105

5.3 Percentage of Launched Particles Reaching the Entire Surface...........................114

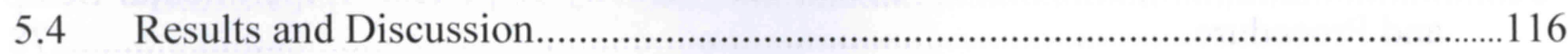

5.4.1 Comparison with Computer Simulation of Refs. [10,12,13]..............................116

5.4.2 Qualitative Comparison with Experimental Observations.....................................126

5.4.3 Advantages over the Computer Simulation, Experimental Methods and Previous Analytical Models.

Chapter 6 Model Limitations.

6.1 Modified Analytical Models for Non-divergent Jets............................................127

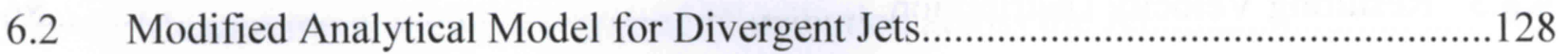

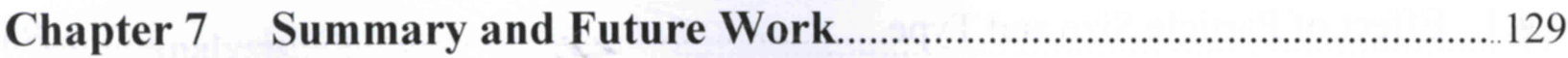

Appendix A Measured $M\left(R^{*}\right) / M(0)$ Values, used in Obtaining the Particle Spatial Distribution across the Jet, along with measured Flow Rates (Corresponding to Section 3.3) 131

Appendix B Measured $\delta\left(r^{*}\right) / \delta(0)$ and Calculated $v^{*}\left(r^{*}\right)$ Values, along with Measured $k$ Values (Selected Cases) used in Obtaining $v^{*}\left(r^{*}\right)$ (Corresponding to Section 4.3).

References 


\section{List of Tables}

Table 2.1: Variables used for comparison of analytical models with computer simulation

Table 3.1: Particle spatial distribution experimental summary and corresponding focus coefficients $(\beta$ 's).

Table 5.1: Variables used for comparison of analytical model with computer simulation. 118

Table A-1a: Measured $M\left(R^{*}\right) / M(0)$ vs. $r^{*}$ for $10 \mu \mathrm{m}$ angular aluminum oxide blasted at $d=30$ $\mathrm{mm}$ for $120 \mathrm{~s}$ at a pressure of $200 \mathrm{kPa}$ and a flow rate of $3.97 \mathrm{~g} / \mathrm{min}$ using $0.76 \mathrm{~mm}$ round nozzle and an $r_{c}$ of $0.6 \mathrm{~mm}$ (corresponding to Figure 3.4).

Table A-1b: Measured flow rate for $10 \mu \mathrm{m}$ angular aluminum oxide blasted at $d=30 \mathrm{~mm}$ for $120 \mathrm{~s}$ at a pressure of $200 \mathrm{kPa}$ using $0.76 \mathrm{~mm}$ round nozzle and an $r_{c}$ of $0.6 \mathrm{~mm}$ (corresponding to Figure 3.4).

Table A-2a: Measured $M\left(R^{*}\right) / M(0)$ vs. $r^{*}$ for $25 \mu \mathrm{m}$ angular aluminum oxide blasted at $d=30$ $\mathrm{mm}$ for $90 \mathrm{~s}$ at a pressure of $200 \mathrm{kPa}$ and a flow rate of $2.83 \mathrm{~g} / \mathrm{min}$ using $0.76 \mathrm{~mm}$ round nozzle and an $r_{c}$ of $0.23 \mathrm{~mm}$ (corresponding to Figure 3.5)....

Table A-2b: Measured flow rate for $25 \mu \mathrm{m}$ angular aluminum oxide blasted at $d=30 \mathrm{~mm}$ for 90 $\mathrm{s}$ at a pressure of $200 \mathrm{kPa}$ using $0.76 \mathrm{~mm}$ round nozzle and an $r_{c}$ of $0.23 \mathrm{~mm}$ (corresponding to Figure 3.5).

Table A-3a: Measured $M\left(R^{*}\right) / M(0)$ vs. $r^{*}$ for $50 \mu \mathrm{m}$ silica glass beads blasted at $d=20 \mathrm{~mm}$ for $120 \mathrm{~s}$ at a pressure of $200 \mathrm{kPa}$ and flow rate of $1.17 \mathrm{~g} / \mathrm{min}$ using $0.76 \mathrm{~mm}$ round nozzle and an $r_{c}$ of $0.23 \mathrm{~mm}$ (corresponding to Figure 3.6).

Table A-3b: Measured flow rate for $50 \mu \mathrm{m}$ silica glass beads blasted at $d=20 \mathrm{~mm}$ for $120 \mathrm{~s}$ at a pressure of $200 \mathrm{kPa}$ using $0.76 \mathrm{~mm}$ round nozzle and an $r_{c}$ of $0.23 \mathrm{~mm}$ (corresponding to Figure 3.6).

Table A-4a: Measured $M\left(R^{*}\right) / M(0)$ vs. $r^{*}$ for $50 \mu \mathrm{m}$ silica glass beads blasted at $d=30 \mathrm{~mm}$ for $120 \mathrm{~s}$ at a pressure of $200 \mathrm{kPa}$ and flow rate of $1.17 \mathrm{~g} / \mathrm{min}$ using $0.76 \mathrm{~mm}$ round nozzle and an $r_{c}$ of $0.23 \mathrm{~mm}$ (corresponding to Figure 3.6).

Table A-4b: Measured flow rate for $50 \mu \mathrm{m}$ silica glass beads blasted at $d=30 \mathrm{~mm}$ for $120 \mathrm{~s}$ at a pressure of $200 \mathrm{kPa}$ using $0.76 \mathrm{~mm}$ round nozzle and an $r_{c}$ of $0.23 \mathrm{~mm}$ (corresponding to Figure 3.6). 
Table A-5a: Measured $M\left(R^{*}\right) / M(0)$ vs. $r^{*}$ for $50 \mu \mathrm{m}$ silica glass beads blasted at $d=20 \mathrm{~mm}$ for $120 \mathrm{~s}$ at a pressure of $200 \mathrm{kPa}$ and flow rate of $0.59 \mathrm{~g} / \mathrm{min}$ using $0.76 \mathrm{~mm}$ round nozzle and an $r_{c}$ of $0.23 \mathrm{~mm}$ (corresponding to Figure 3.6)...

Table A-5b: Measured flow rate for $50 \mu \mathrm{m}$ silica glass beads blasted at $d=20 \mathrm{~mm}$ for $120 \mathrm{~s}$ at a pressure of $200 \mathrm{kPa}$ using $0.76 \mathrm{~mm}$ round nozzle and an $r_{c}$ of $0.23 \mathrm{~mm}$ (corresponding to Figure 3.6). 134

Table A-6a: Measured $M\left(R^{*}\right) / M(0)$ vs. $r^{*}$ for $25 \mu \mathrm{m}$ angular aluminum oxide blasted at $d=10$ $\mathrm{mm}$ for $180 \mathrm{~s}$ at a pressure of $200 \mathrm{kPa}$ and flow rate of $0.36 \mathrm{~g} / \mathrm{min}$ using $0.46 \mathrm{~mm}$ H.P. round nozzle and an $r_{c}$ of $0.23 \mathrm{~mm}$ (corresponding to Figure 3.7). .134

Table A-6b: Measured flow rate for $25 \mu \mathrm{m}$ angular aluminum oxide blasted at $d=10 \mathrm{~mm}$ for $180 \mathrm{~s}$ at a pressure of $200 \mathrm{kPa}$ using $0.46 \mathrm{~mm} \mathrm{H.P.} \mathrm{round} \mathrm{nozzle} \mathrm{and} \mathrm{an} r_{c}$ of $0.23 \mathrm{~mm}$ (corresponding to Figure 3.7).... 134

Table A-7a: Measured $M\left(R^{*}\right) / M(0)$ vs. $r^{*}$ for $25 \mu \mathrm{m}$ angular aluminum oxide blasted at $d=30$ $\mathrm{mm}$ for $60 \mathrm{~s}$ at a pressure of $200 \mathrm{kPa}$ and flow rate of $4.75 \mathrm{~g} / \mathrm{min}$ using $1.5 \mathrm{~mm} \mathrm{H.P.} \mathrm{round}$ nozzle and an $r_{c}$ of $0.23 \mathrm{~mm}$ (corresponding to Figure 3.8 )....... 135

Table A-7b: Measured flow rate for $25 \mu \mathrm{m}$ angular aluminum oxide blasted at $d=30 \mathrm{~mm}$ for 60 $\mathrm{s}$ at a pressure of $200 \mathrm{kPa}$ using $1.5 \mathrm{~mm} \mathrm{H.P.} \mathrm{round} \mathrm{nozzle} \mathrm{and} \mathrm{an} r_{c}$ of $0.23 \mathrm{~mm}$ (corresponding to Figure 3.8). 135

Table A-8a: Measured $M\left(R^{*}\right) / M(0)$ vs. $r^{*}$ for $50 \mu \mathrm{m}$ silica glass beads blasted at $d=10 \mathrm{~mm}$ for $180 \mathrm{~s}$ at a pressure of $200 \mathrm{kPa}$ and flow rate of $0.23 \mathrm{~g} / \mathrm{min}$ using $0.46 \mathrm{~mm}$ H.P. round nozzle and an $r_{c}$ of $0.23 \mathrm{~mm}$ (corresponding to Figure 3.9). .135

Table A-8b: Measured flow rate for $50 \mu \mathrm{m}$ silica glass beads blasted at $d=10 \mathrm{~mm}$ for $180 \mathrm{~s}$ at a pressure of $200 \mathrm{kPa}$ using $0.46 \mathrm{~mm} \mathrm{H.P}$. round nozzle and an $r_{c}$ of $0.23 \mathrm{~mm}$ (corresponding to Figure 3.9). 136

Table A-9a: Measured $M\left(R^{*}\right) / M(0)$ vs. $r^{*}$ for $50 \mu \mathrm{m}$ silica glass beads blasted at $d=20 \mathrm{~mm}$ for $240 \mathrm{~s}$ at a pressure of $200 \mathrm{kPa}$ and flow rate of $0.23 \mathrm{~g} / \mathrm{min}$ using $0.46 \mathrm{~mm} \mathrm{H} . P$. round nozzle and an $r_{c}$ of $0.23 \mathrm{~mm}$ (corresponding to Figure 3.9). 136

Table A-9b: Measured flow rate for $50 \mu \mathrm{m}$ silica glass beads blasted at $d=20 \mathrm{~mm}$ for $240 \mathrm{~s}$ at a pressure of $200 \mathrm{kPa}$ using $0.46 \mathrm{~mm} \mathrm{H.P}$. round nozzle and an $r_{c}$ of $0.23 \mathrm{~mm}$ (corresponding to Figure 3.9). 136

Table A-10a: Measured $M\left(R^{*}\right) / M(0)$ vs. $r^{*}$ for $50 \mu \mathrm{m}$ silica glass beads blasted at $d=20 \mathrm{~mm}$ for $60 \mathrm{~s}$ at a pressure of $200 \mathrm{kPa}$ and flow rate of $1.31 \mathrm{~g} / \mathrm{min}$ using $1.5 \mathrm{~mm} \mathrm{H.P.} \mathrm{round} \mathrm{nozzle} \mathrm{and} \mathrm{an}$ $r_{c}$ of $0.23 \mathrm{~mm}$ (corresponding to Figure 3.10). .137 
Table A-10b: Measured flow rate for $50 \mu \mathrm{m}$ silica glass beads blasted at $d=20 \mathrm{~mm}$ for $60 \mathrm{~s}$ at a pressure of $200 \mathrm{kPa}$ using $1.5 \mathrm{~mm}$ H.P. round nozzle and an $r_{c}$ of $0.23 \mathrm{~mm}$ (corresponding to Figure 3.10).

Table A-11a: Measured $M\left(R^{*}\right) / M(0)$ vs. $r^{*}$ for $10 \mu \mathrm{m}$ angular aluminum oxide blasted at $d=30$ $\mathrm{mm}$ for $60 \mathrm{~s}$ at a pressure of $300 \mathrm{kPa}$ and a flow rate of $4.20 \mathrm{~g} / \mathrm{min}$ using $0.76 \mathrm{~mm}$ round nozzle and an $r_{c}$ of $0.6 \mathrm{~mm}$ (corresponding to Figure 3.11).

Table A-11b: Measured flow rate for $10 \mu \mathrm{m}$ angular aluminum oxide blasted at $d=30 \mathrm{~mm}$ for $60 \mathrm{~s}$ at a pressure of $300 \mathrm{kPa}$ using $0.76 \mathrm{~mm}$ round nozzle and an $r_{c}$ of $0.6 \mathrm{~mm}$ (corresponding to Figure 3.11). 138

Table A-12a: Measured $M\left(R^{*}\right) / M(0)$ vs. $r^{*}$ for $25 \mu \mathrm{m}$ angular aluminum oxide blasted at $d=30$ $\mathrm{mm}$ for $60 \mathrm{~s}$ at a pressure of $300 \mathrm{kPa}$ and a flow rate of $3.10 \mathrm{~g} / \mathrm{min}$ using $0.76 \mathrm{~mm}$ round nozzle and an $r_{c}$ of $0.23 \mathrm{~mm}$ (corresponding to Figure 3.12). 138

Table A-12b: Measured flow rate for $25 \mu \mathrm{m}$ angular aluminum oxide blasted at $d=30 \mathrm{~mm}$ for $60 \mathrm{~s}$ at a pressure of $300 \mathrm{kPa}$ using $0.76 \mathrm{~mm}$ round nozzle and an $r_{c}$ of $0.23 \mathrm{~mm}$ (corresponding to Figure 3.12). .138

Table A-13a: Measured $M\left(R^{*}\right) / M(0)$ vs. $r^{*}$ for $50 \mu \mathrm{m}$ silica glass beads blasted at $d=20 \mathrm{~mm}$ for $90 \mathrm{~s}$ at a pressure of $300 \mathrm{kPa}$ and a flow rate of $1.34 \mathrm{~g} / \mathrm{min}$ using $0.76 \mathrm{~mm}$ round nozzle and an $r_{c}$ of $0.23 \mathrm{~mm}$ (corresponding to Figure 3.13 )....

Table A-13b: Measured flow rate for $50 \mu \mathrm{m}$ silica glass beads blasted at $d=20 \mathrm{~mm}$ for $90 \mathrm{~s}$ at a pressure of $300 \mathrm{kPa}$ using $0.76 \mathrm{~mm}$ round nozzle and an $r_{c}$ of $0.23 \mathrm{~mm}$ (corresponding to Figure $3.13)$. 139

Table A-14a: Measured $M\left(R^{*}\right) / M(0)$ vs. $r^{*}$ for $50 \mu \mathrm{m}$ silica glass beads blasted at $d=30 \mathrm{~mm}$ for $90 \mathrm{~s}$ at a pressure of $300 \mathrm{kPa}$ and a flow rate of $1.34 \mathrm{~g} / \mathrm{min}$ using $0.76 \mathrm{~mm}$ round nozzle and an $r_{c}$ of $0.23 \mathrm{~mm}$ (corresponding to Figure 3.13 ).

Table A-14b: Measured flow rate for $50 \mu \mathrm{m}$ silica glass beads blasted at $d=30 \mathrm{~mm}$ for $90 \mathrm{~s}$ at a pressure of $300 \mathrm{kPa}$ using $0.76 \mathrm{~mm}$ round nozzle and an $r_{c}$ of $0.23 \mathrm{~mm}$ (corresponding to Figure $3.13)$ 140

Table A-15a: Measured $M\left(X^{*}\right) / M(0)$ vs. $x^{*}$ for $50 \mu \mathrm{m}$ silica glass beads blasted at $d=40 \mathrm{~mm}$ for $120 \mathrm{~s}$ at a pressure of $200 \mathrm{kPa}$ and a flow rate of $1.21 \mathrm{~g} / \mathrm{min}$ using $3.2 \times 0.2 \mathrm{~mm}$ rectangular nozzle and an $r_{c}$ of $0.23 \mathrm{~mm}$ (scanning direction along $0.2 \mathrm{~mm}$ ) (corresponding to Figure $3.14)$. 140

Table A-15b: Measured flow rate for $50 \mu \mathrm{m}$ silica glass beads blasted at $d=40 \mathrm{~mm}$ for $120 \mathrm{~s}$ at a pressure of $200 \mathrm{kPa}$ using $3.2 \times 0.2 \mathrm{~mm}$ rectangular nozzle and an $r_{c}$ of $0.23 \mathrm{~mm}$ (scanning direction along $0.2 \mathrm{~mm}$ ) (corresponding to Figure 3.14)..... 140 
Table A-16a: Measured $M\left(Y^{*}\right) / M(0)$ vs. $y^{*}$ for $50 \mu \mathrm{m}$ silica glass beads blasted at $d=40 \mathrm{~mm}$ for $60 \mathrm{~s}$ at a pressure of $200 \mathrm{kPa}$ and a flow rate of $1.21 \mathrm{~g} / \mathrm{min}$ using $3.2 \times 0.2 \mathrm{~mm}$ rectangular nozzle and an $r_{c}$ of $0.23 \mathrm{~mm}$ (scanning direction along $3.2 \mathrm{~mm}$ ) (corresponding to Figure 3.14).

Table A-16b: Measured flow rate for $50 \mu \mathrm{m}$ silica glass beads blasted at $d=40 \mathrm{~mm}$ for $60 \mathrm{~s}$ at a pressure of $200 \mathrm{kPa}$ using $3.2 \times 0.2 \mathrm{~mm}$ rectangular nozzle and an $r_{c}$ of $0.23 \mathrm{~mm}$ (scanning direction along $3.2 \mathrm{~mm}$ ) (corresponding to Figure 3.14).

Table A-17a: Measured $M\left(X^{*}\right) / M(0)$ vs. $x^{*}$ for $50 \mu \mathrm{m}$ silica glass beads blasted at $d=40 \mathrm{~mm}$ for $120 \mathrm{~s}$ at a pressure of $200 \mathrm{kPa}$ and a flow rate of $1.78 \mathrm{~g} / \mathrm{min}$ using $3.8 \times 0.3 \mathrm{~mm}$ rectangular nozzle and an $r_{c}$ of $0.23 \mathrm{~mm}$ (scanning direction along $0.3 \mathrm{~mm}$ ) (corresponding to Figure 3.15).

Table A-17b: Measured flow rate for $50 \mu \mathrm{m}$ silica glass beads blasted at $d=40 \mathrm{~mm}$ for $120 \mathrm{~s}$ at a pressure of $200 \mathrm{kPa}$ using $3.8 \times 0.3 \mathrm{~mm}$ rectangular nozzle and an $r_{c}$ of $0.23 \mathrm{~mm}$ (scanning direction along $0.3 \mathrm{~mm}$ ) (corresponding to Figure 3.15 ).

Table A-18a: Measured $M\left(Y^{*}\right) / M(0)$ vs. $y^{*}$ for $50 \mu \mathrm{m}$ silica glass beads blasted at $d=40 \mathrm{~mm}$ for $120 \mathrm{~s}$ at a pressure of $200 \mathrm{kPa}$ and a flow rate of $1.78 \mathrm{~g} / \mathrm{min}$ using $3.8 \times 0.3 \mathrm{~mm}$ rectangular nozzle and an $r_{c}$ of $0.23 \mathrm{~mm}$ (scanning direction along $3.8 \mathrm{~mm}$ ) (corresponding to Figure $3.15)$ 143

Table A-18b: Measured flow rate for $50 \mu \mathrm{m}$ silica glass beads blasted at $d=40 \mathrm{~mm}$ for $120 \mathrm{~s}$ at a pressure of $200 \mathrm{kPa}$ using $3.8 \times 0.3 \mathrm{~mm}$ rectangular nozzle and an $r_{c}$ of $0.23 \mathrm{~mm}$ (scanning direction along $3.8 \mathrm{~mm}$ ) (corresponding to Figure 3.15). 143

Table B-1a: Measured $\delta\left(r^{*}\right) / \delta(0)$ (erosion time $=10 \mathrm{~s}$ ) and calculated $v^{*}\left(r^{*}\right)$ vs. $r^{*}($ corresponding to Figure 3.4 of Section 3.3, analysis of Section 4.2, and Figure 4.5 of Section 4.3) using $k=1.57$ (Figure 4.2) for $10 \mu \mathrm{m}$ angular aluminum oxide blasted at $d=30 \mathrm{~mm}$ at a pressure of $200 \mathrm{kPa}$ and a flow rate of $3.97 \mathrm{~g} / \mathrm{min}$ using $0.76 \mathrm{~mm}$ round nozzle. 144

Table B-1b: Measured normalized average erosion rate, $E^{*}$, as a function of $\sin (\alpha)$ (corresponding to Figure 4.2 and Table B-1a). Best fit velocity exponent $k=1.57$. Erosion of Borofloat glass by $10 \mu \mathrm{m}$ aluminum oxide particles blasted at $d=30 \mathrm{~mm}$ for $60 \mathrm{~s}$ at a pressure of $200 \mathrm{kPa}$ and a flow rate of $3.97 \mathrm{~g} / \mathrm{min}$ using $0.76 \mathrm{~mm}$ round nozzle. 
Table B-2: Measured $\delta\left(r^{*}\right) / \delta(0)$ (erosion time $=10 \mathrm{~s}$ ) and calculated $v^{*}\left(r^{*}\right)$ vs. $r^{*}$ (corresponding to Figure 3.5 of Section 3.3, analysis of Section 4.2, and Figure 4.6 of Section 4.3) using $k=1.43$ (from [25]) for $25 \mu \mathrm{m}$ angular aluminum oxide blasted at $d=30 \mathrm{~mm}$ at a pressure of $200 \mathrm{kPa}$ and a flow rate of $2.83 \mathrm{~g} / \mathrm{min}$ using $0.76 \mathrm{~mm}$ round nozzle.............................. 145

Table B-3: Measured $\delta\left(r^{*}\right) / \delta(0)$ (erosion time $\left.=180 \mathrm{~s}\right)$ and calculated $v^{*}\left(r^{*}\right)$ vs. $r^{*}($ corresponding to Figure 3.6 of Section 3.3, analysis of Section 4.2, and Figure 4.7 of Section 4.3) using $k=1.6$ and $k=2.7$ for $50 \mu \mathrm{m}$ silica glass beads blasted at $d=20 \mathrm{~mm}$ at a pressure of $200 \mathrm{kPa}$ and a flow

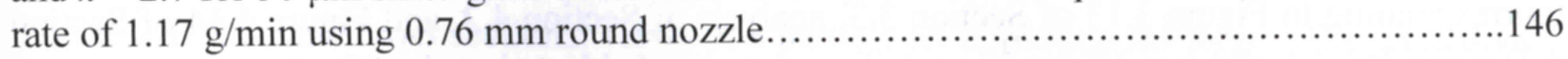

Table B-4: Measured $\delta\left(r^{*}\right) / \delta(0)$ (erosion time $=10 \mathrm{~s}$ ) and calculated $v^{*}\left(r^{*}\right)$ vs. $r^{*}$ (corresponding to Figure 3.7 of Section 3.3, analysis of Section 4.2, and Figure 4.8 of Section 4.3) using $k=1.43$ (from [25]) for $25 \mu \mathrm{m}$ angular aluminum oxide blasted at $d=10 \mathrm{~mm}$ at a pressure of $200 \mathrm{kPa}$ and a flow rate of $0.36 \mathrm{~g} / \mathrm{min}$ using $0.46 \mathrm{~mm}$ H.P. round nozzle

Table B-5: Measured $\delta\left(r^{*}\right) / \delta(0)$ (erosion time $=2 \mathrm{~s}$ ) and calculated $v^{*}\left(r^{*}\right)$ vs. $r^{*}$ (corresponding to Figure 3.8 of Section 3.3, analysis of Section 4.2, and Figure 4.9 of Section 4.3) using $k=1.43$ (from [25]) for $25 \mu \mathrm{m}$ angular aluminum oxide blasted at $d=30 \mathrm{~mm}$ at a pressure of $200 \mathrm{kPa}$ and a flow rate of $4.75 \mathrm{~g} / \mathrm{min}$ using $1.5 \mathrm{~mm} \mathrm{H.P.} \mathrm{round} \mathrm{nozzle...........................} 147$

Table B-6: Measured $\delta\left(r^{*}\right) / \delta(0)$ (erosion time $=300 \mathrm{~s}$ ) and calculated $v^{*}\left(r^{*}\right)$ vs. $r^{*}$ (corresponding to Figure 3.9 of Section 3.3, analysis of Section 4.2, and Figure 4.10 of Section 4.3) using $k=1.6$ and $k=2.7$ for $50 \mu \mathrm{m}$ silica glass beads blasted at $d=10 \mathrm{~mm}$ at a pressure of $200 \mathrm{kPa}$ and a flow rate of $0.23 \mathrm{~g} / \mathrm{min}$ using $0.46 \mathrm{~mm}$ H.P. round nozzle..................................... 148

Table B-7: Measured $\delta\left(r^{*}\right) / \delta(0)$ (erosion time $=10 \mathrm{~s}$ ) and calculated $v^{*}\left(r^{*}\right)$ vs. $r^{*}$ (corresponding to Figure 3.10 of Section 3.3, analysis of Section 4.2, and Figure 4.11 of Section 4.3) using $k=$ 1.6 and $k=2.7$ for $50 \mu \mathrm{m}$ silica glass beads blasted at $d=20 \mathrm{~mm}$ at a pressure of $200 \mathrm{kPa}$ and a flow rate of $1.31 \mathrm{~g} / \mathrm{min}$ using $1.5 \mathrm{~mm}$ H.P. round nozzle.

Table B-8a: Measured $\delta\left(r^{*}\right) / \delta(0)$ (erosion time $=5 \mathrm{~s}$ ) and calculated $v^{*}\left(r^{*}\right)$ vs. $r^{*}$ (corresponding to Figure 3.11 of Section 3.3, analysis of Section 4.2, and Figure 4.12 of Section 4.3) using $k=$ 1.44 (Figure 4.3) for $10 \mu \mathrm{m}$ angular aluminum oxide blasted at $d=30 \mathrm{~mm}$ at a pressure of 300 $\mathrm{kPa}$ and a flow rate of $4.20 \mathrm{~g} / \mathrm{min}$ using $0.76 \mathrm{~mm}$ round nozzle.......................... 149

Table B-8b: Measured normalized average erosion rate, $E^{*}$, as a function of $\sin (\alpha)$ (corresponding to Figure 4.3 and Table B-8a). Best fit velocity exponent $k=1.44$. Erosion of Borofloat glass by $10 \mu \mathrm{m}$ aluminum oxide particles blasted at $d=30 \mathrm{~mm}$ for $60 \mathrm{~s}$ at a pressure of $300 \mathrm{kPa}$ and a flow rate of $4.20 \mathrm{~g} / \mathrm{min}$ using $0.76 \mathrm{~mm}$ round nozzle.

Table B-9a: Measured $\delta\left(r^{*}\right) / \delta(0)$ (erosion time $=3$ s) and calculated $v^{*}\left(r^{*}\right)$ vs. $r^{*}$ (corresponding to Figure 3.12 of Section 3.3, analysis of Section 4.2, and Figure 4.13 of Section 4.3) using $k=$ 1.59 (Figure 4.4) for $25 \mu \mathrm{m}$ angular aluminum oxide blasted at $d=30 \mathrm{~mm}$ at a pressure of 300 $\mathrm{kPa}$ and a flow rate of $3.10 \mathrm{~g} / \mathrm{min}$ using $0.76 \mathrm{~mm}$ round nozzle. 151 
Table B-9b: Measured normalized average erosion rate, $E^{*}$, as a function of $\sin (\alpha)$ (corresponding to Figure 4.4 and Table B-9a). Best fit velocity exponent $k=1.59$. Erosion of Borofloat glass by $25 \mu \mathrm{m}$ aluminum oxide particles blasted at $d=30 \mathrm{~mm}$ for $60 \mathrm{~s}$ at a pressure of $300 \mathrm{kPa}$ and a flow rate of $3.10 \mathrm{~g} / \mathrm{min}$ using $0.76 \mathrm{~mm}$ round nozzle.

Table B-10: Measured $\delta\left(r^{*}\right) / \delta(0)$ (erosion time $=150 \mathrm{~s}$ ) and calculated $v^{*}\left(r^{*}\right)$ vs. $r^{*}$ (corresponding to Figure 3.13 of Section 3.3, analysis of Section 4.2, and Figure 4.14 of Section 4.3) using $k=1.6$ and $k=2.7$ for $50 \mu \mathrm{m}$ silica glass beads blasted at $d=20 \mathrm{~mm}$ at a pressure of $300 \mathrm{kPa}$ and a flow rate of $1.34 \mathrm{~g} / \mathrm{min}$ using $0.76 \mathrm{~mm}$ round nozzle. 152

Table B-11: Measured $\delta\left(r^{*}\right) / \delta(0)$ (erosion time $=240 \mathrm{~s}$ ) and calculated $v^{*}\left(r^{*}\right)$ vs. $r^{*}$ (corresponding to Figure 3.6 of Section 3.3, analysis of Section 4.2, and Figure 4.15 of Section 4.3) using $k=1.6$ and $k=2.7$ for $50 \mu \mathrm{m}$ silica glass beads blasted at $d=20 \mathrm{~mm}$ at a pressure of $200 \mathrm{kPa}$ and a flow rate of $0.59 \mathrm{~g} / \mathrm{min}$ using $0.76 \mathrm{~mm}$ round nozzle 


\section{List of Figures}

Figure 1.1: Geometric definitions for a plume of particles, emanating from a point source ("nozzle"), and arriving to a surface at a radius $r$.

Figure 2.1: Side and cross sectional views of a particle jet impacting the target. The jet is divided into $i$ tubular regions, each containing particles located at a radial coordinate $r_{i}$, and a cylinder containing particles located at a radial coordinate $r_{0}$

Figure 2.2: Collision between an arriving and departing particle (adapted from Ref. [8]) 22

Figure 2.3: (a) Particle scattering, collision zone, and intersection of trajectories through a region of possible collisions; (b) Close up view of collision zone. .28

Figure 2.4: Estimation of the number of intersections between an incident trajectory and rebounding trajectories in the entire rebounding jet cross section.

Figure 2.5: Effect of stream density, $\rho_{s}$ (lower range), on proportion of launched particles arriving to surface without having undergone a collision, $\Omega_{z} / \Omega_{a}$, for various models. Frictionless $(f=0)$ and elastic $\left(e_{p s}=1\right)$ particle-surface collisions assumed (Table 2.1$)$

Figure 2.6: Effect of stream density, $\rho_{s}$ (higher range), on proportion of launched particles arriving to surface without having undergone a collision, $\Omega_{z} / \Omega_{a}$, for various models. Frictionless $(f=0)$ and elastic $\left(e_{p s}=1\right)$ particle-surface collisions assumed (Table 2.1)

Figure 2.7: Effect of incident angle, $\alpha$, on proportion of launched particles arriving to surface without having undergone a collision, $\Omega_{z} / \Omega_{a}$, for various models. Frictionless $(f=0)$ and elastic $\left(e_{p s}=1\right)$ particle-surface collisions assumed (Table 2.1).

Figure 2.8: Effect of stream density, $\rho_{s}$ (lower range), on proportion of launched particles arriving to surface without having undergone a collision, $\Omega_{z} / \Omega_{a}$, for various models. Frictionless $(f=0)$ and inelastic $\left(e_{p s}=0.5\right)$ particle-surface collisions assumed (Table 2.1)....

Figure 2.9: Effect of stream density, $\rho_{s}$ (lower range), on proportion of launched particles arriving to surface without having undergone a collision, $\Omega_{z} / \Omega_{a}$, for various models. Frictional $(f$ $=0.5)$ and elastic $\left(e_{p s}=1\right)$ particle-surface collisions assumed (Table 2.1).

Figure 3.1: Schematic of microblaster. 
Figure 3.2: Schematic of experimental setup (side view).

Figure 3.3: Particle collection apparatus for measurement of particle spatial distribution across jet (top view along with the jet cross sectional view)...... .48

Figure 3.4: Measured $M\left(R^{*}\right) / M(0)$ vs. $r^{*}$ for $10 \mu \mathrm{m}$ angular aluminum oxide blasted at $d=30 \mathrm{~mm}$ for $120 \mathrm{~s}$ at pressure of $200 \mathrm{kPa}$ and flow rate of $3.97 \mathrm{~g} / \mathrm{min}$ using $0.76 \mathrm{~mm}$ round nozzle and $r_{c}=$ $0.6 \mathrm{~mm}$ ( $\square, \diamond$-repeat). — : Equation (3.5) using Weibull distribution (equation (3.6)) with $\beta=16$ with avg. $\mathrm{R}^{2}=0.98$ for the experimental data fit ..53

Figure 3.5: Measured $M\left(R^{*}\right) / M(0)$ vs. $r^{*}$ for $25 \mu \mathrm{m}$ angular aluminum oxide blasted at $d=30 \mathrm{~mm}$ for $90 \mathrm{~s}$ at pressure of $200 \mathrm{kPa}$ and flow rate of $2.83 \mathrm{~g} / \mathrm{min}$ using $0.76 \mathrm{~mm}$ round nozzle and $r_{c}=$ $0.23 \mathrm{~mm}$ ( $\square, \diamond$-repeat). —: Equation (3.5) using Weibull distribution (equation (3.6)) with $\beta=15$ with avg. $\mathrm{R}^{2}=0.97$ for the experimental data fit.

Figure 3.6: Measured $M\left(R^{*}\right) / M(0)$ vs. $r^{*}$ for $50 \mu \mathrm{m}$ silica glass beads blasted for $120 \mathrm{~s}$ at pressure of $200 \mathrm{kPa}$ using $0.76 \mathrm{~mm}$ round nozzle and $r_{c}=0.23 \mathrm{~mm}$ (口,, -repeat: $d=20 \mathrm{~mm}$, flow rate $=$ $1.17 \mathrm{~g} / \mathrm{min}$, avg. $\mathrm{R}^{2}=0.97 ; \mathrm{x}$, +-repeat: $d=30 \mathrm{~mm}$, flow rate $=1.17 \mathrm{~g} / \mathrm{min}$, avg. $\mathrm{R}^{2}=0.96 ; \circ, \Delta-$ repeat: $d=20 \mathrm{~mm}$, flow rate $=0.59 \mathrm{~g} / \mathrm{min}$, avg. $\left.\mathrm{R}^{2}=0.97\right)$. - : Equation (3.5) using Weibull distribution (equation (3.6)) with $\beta=14$ .54

Figure 3.7: Measured $M\left(R^{*}\right) / M(0)$ vs. $r^{*}$ for $25 \mu \mathrm{m}$ angular aluminum oxide blasted at $d=10 \mathrm{~mm}$ for $180 \mathrm{~s}$ at pressure of $200 \mathrm{kPa}$ and flow rate of $0.36 \mathrm{~g} / \mathrm{min}$ using $0.46 \mathrm{~mm}$ H.P. round nozzle and $r_{c}=0.23 \mathrm{~mm}$ (口, $\triangleright$-repeat). - : Equation (3.9) $\left(F_{3}{ }^{P}=-0.025, F_{3}{ }^{S}=1.9, A=0.09\right)$ using Weibull distributions (equations (3.6) and (3.10)) with $\beta=12$ with avg. $\mathrm{R}^{2}=0.99$ for the experimental data fit....

Figure 3.8: Measured $M\left(R^{*}\right) / M(0)$ vs. $r^{*}$ for $25 \mu \mathrm{m}$ angular aluminum oxide blasted at $d=30 \mathrm{~mm}$ for $60 \mathrm{~s}$ at pressure of $200 \mathrm{kPa}$ and flow rate of $4.75 \mathrm{~g} / \mathrm{min}$ using $1.5 \mathrm{~mm} \mathrm{H.P.} \mathrm{round} \mathrm{nozzle} \mathrm{and}$ $r_{c}=0.23 \mathrm{~mm}$ ( $\square, \diamond$-repeat). - - Equation (3.5) using Weibull distribution (equation (3.6)) with $\beta$ $=20$ with avg. $\mathrm{R}^{2}=0.99$ for the experimental data fit.

Figure 3.9: Measured $M\left(R^{*}\right) / M(0)$ vs. $r^{*}$ for $50 \mu \mathrm{m}$ silica glass beads blasted at pressure of 200 $\mathrm{kPa}$ and flow rate of $0.23 \mathrm{~g} / \mathrm{min}$ using $0.46 \mathrm{~mm}$ H.P. round nozzle and $r_{c}=0.23 \mathrm{~mm}$ ( $\square, \diamond$-repeat: $d=10 \mathrm{~mm}$, exposure time $=180 \mathrm{~s}$, avg. $\mathrm{R}^{2}=0.99 ; \mathrm{x}$, + -repeat: $d=20 \mathrm{~mm}$, exposure time $=240 \mathrm{~s}$, avg. $\left.\mathrm{R}^{2}=0.94\right)$. - : Equation (3.9) $\left(F_{3}{ }^{P}=-0.04, F_{3}{ }^{S}=1.8, A=0.1\right)$ using Weibull distributions (equation (3.6) and (3.10)) with $\beta=9$. .56

Figure 3.10: Measured $M\left(R^{*}\right) / M(0)$ vs. $r^{*}$ for $50 \mu \mathrm{m}$ silica glass beads blasted at $d=20 \mathrm{~mm}$ for $60 \mathrm{~s}$ at pressure of $200 \mathrm{kPa}$ and flow rate of $1.31 \mathrm{~g} / \mathrm{min}$ using $1.5 \mathrm{~mm} \mathrm{H.P.} \mathrm{round} \mathrm{nozzle} \mathrm{and} r_{c}=$ $0.23 \mathrm{~mm}$ ( $\square, \diamond$-repeat). —: Equation (3.5) using Weibull distribution (equation (3.6)) with $\beta=18$ with avg. $\mathrm{R}^{2}=0.99$ for the experimental data fit 
Figure 3.11: Measured $M\left(R^{*}\right) / M(0)$ vs. $r^{*}$ for $10 \mu \mathrm{m}$ angular aluminum oxide blasted at $d=30$ $\mathrm{mm}$ for $60 \mathrm{~s}$ at pressure of $300 \mathrm{kPa}$ and flow rate of $4.20 \mathrm{~g} / \mathrm{min}$ using $0.76 \mathrm{~mm}$ round nozzle and $r_{c}=0.6 \mathrm{~mm}(\square, \diamond$-repeat). —: Equation (3.5) using Weibull distribution (equation (3.6)) with $\beta=$ 20 with avg. $\mathrm{R}^{2}=0.96$ for the experimental data fit.

Figure 3.12: Measured $M\left(R^{*}\right) / M(0)$ vs. $r^{*}$ for $25 \mu \mathrm{m}$ angular aluminum oxide blasted at $d=30$ $\mathrm{mm}$ for $60 \mathrm{~s}$ at pressure of $300 \mathrm{kPa}$ and flow rate of $3.10 \mathrm{~g} / \mathrm{min}$ using $0.76 \mathrm{~mm}$ round nozzle and $r_{c}=0.23 \mathrm{~mm}$ ( $\square, \diamond$-repeat). —: Equation (3.5) using Weibull distribution (equation (3.6)) with $\beta$ $=17$ with avg. $\mathrm{R}^{2}=0.99$ for the experimental data fit

Figure 3.13: Measured $M\left(R^{*}\right) / M(0)$ vs. $r^{*}$ for $50 \mu \mathrm{m}$ silica glass beads blasted for $90 \mathrm{~s}$ at pressure of $300 \mathrm{kPa}$ and flow rate of $1.34 \mathrm{~g} / \mathrm{min}$ using $0.76 \mathrm{~mm}$ round nozzle and $r_{c}=0.23 \mathrm{~mm}(\square, \nabla-$ repeat: $d=20 \mathrm{~mm}$; x,+-repeat: $d=30 \mathrm{~mm}$ ). - Equation (3.5) using Weibull distribution (equation (3.6)) with $\beta=12$ with avg. $\mathrm{R}^{2}=0.99$ for the experimental data fit.................58

Figure 3.14: Measured $M\left(X^{*}\right) / M(0)$ vs. $x^{*}$ and $M\left(Y^{*}\right) / M(0)$ vs. $y^{*}$ for $50 \mu \mathrm{m}$ silica glass beads blasted at $d=40 \mathrm{~mm}$ at pressure of $200 \mathrm{kPa}$ and flow rate of $1.21 \mathrm{~g} / \mathrm{min}$ using $3.2 \times 0.2 \mathrm{~mm}$ rectangular nozzle and $r_{c}=0.23 \mathrm{~mm}$ ( $\square, \diamond$-repeat: Scanning direction along $x$-axis (parallel to $w=$ $0.2 \mathrm{~mm}$ ), time $=120 \mathrm{~s}$; $\mathrm{x},+-$-repeat: Scanning direction along $y$-axis (parallel to $l=3.2 \mathrm{~mm}$ ), time $=60 \mathrm{~s})$. - - Equation (3.11) $\left(F_{2}{ }^{P}=0.04, F_{3}{ }^{S}=1.7, F_{4}{ }^{S}=0.3, A=0.0375\right.$, and $\left.B=0.0875\right)$ using Weibull distributions (equations (3.12), (3.13) and (3.14)) with $\beta=13$ with avg. $\mathrm{R}^{2}=0.98$ for the experimental data fit. ----: Equation (3.16) using Weibull distribution (equation (3.17)) with $\beta=$ 13 with avg. $\mathrm{R}^{2}=0.98$ for the experimental data fit

Figure 3.15: Measured $M\left(X^{*}\right) / M(0)$ vs. $x^{*}$ and $M\left(Y^{*}\right) / M(0)$ vs. $y^{*}$ for $50 \mu \mathrm{m}$ silica glass beads blasted for $120 \mathrm{~s}$ at $d=40 \mathrm{~mm}$ at pressure of $200 \mathrm{kPa}$ and flow rate of $1.78 \mathrm{~g} / \mathrm{min}$ using $3.8 \times 0.3$ $\mathrm{mm}$ rectangular nozzle and $r_{c}=0.23 \mathrm{~mm}(\square, \diamond$-repeat: Scanning direction along $x$-axis (parallel to $w=0.3 \mathrm{~mm}$ ); $\mathrm{x}$, +-repeat: Scanning direction along $y$-axis (parallel to $l=3.8 \mathrm{~mm}$ )). - : Equation (3.11) $\left(F_{2}{ }^{P}=0.0325, F_{3}{ }^{S}=1.65, F_{4}{ }^{S}=0.25, A=0.0375\right.$, and $\left.B=0.0875\right)$ using Weibull distributions (equations (3.12), (3.13) and (3.14)) with $\beta=13.5$ with avg. $\mathrm{R}^{2}=0.98$ for the experimental data fit. ----: Equation (3.16) using Weibull distribution (equation (3.17)) with $\beta=$ 13.5 with avg. $\mathrm{R}^{2}=0.99$ for the experimental data fit

Figure 3.16: Nozzle plane view of the inner opening of a rectangular nozzle (shorter side, $w$, and longer side, $l$ ), and the $x$ - and $y$-axes, along with respective center line distances from the jet center to center of collection cylinder, $X$ and $Y$ (analogous to $R$; see Figure 3.3).............62

Figure 4.1: Example of a shallow profile (profile depth, $\delta(r)$, as a function of $r$ ) (corresponding to Table B-2) for $25 \mu \mathrm{m}$ angular aluminum oxide blasted at $d=30 \mathrm{~mm}$ for $10 \mathrm{~s}$ at a pressure of 200 $\mathrm{kPa}$ and a flow rate of $2.83 \mathrm{~g} / \mathrm{min}$ using a $0.76 \mathrm{~mm}$ round nozzle .........................79 
Figure 4.2: Average $E^{*}$ vs. $\sin (\alpha)$ (corresponding to data in Table B-1b). Best fit velocity exponent $k=1.57$. Erosion of Borofloat glass by $10 \mu \mathrm{m}$ aluminum oxide particles blasted at $d=$ $30 \mathrm{~mm}$ for $60 \mathrm{~s}$ at pressure of $200 \mathrm{kPa}$ and flow rate of $3.97 \mathrm{~g} / \mathrm{min}$ using $0.76 \mathrm{~mm}$ round nozzle. - : Power regression fit to experimental data $\left(\mathbf{m}: \mathrm{R}^{2}=0.97\right)$

Figure 4.3: Average $E^{*}$ vs. $\sin (\alpha)$ (corresponding to data in Table B-8b). Best fit velocity exponent $k=1.44$. Erosion of Borofloat glass by $10 \mu \mathrm{m}$ aluminum oxide particles blasted at $d=$ $30 \mathrm{~mm}$ for $60 \mathrm{~s}$ at pressure of $300 \mathrm{kPa}$ and flow rate of $4.20 \mathrm{~g} / \mathrm{min}$ using $0.76 \mathrm{~mm}$ round nozzle.

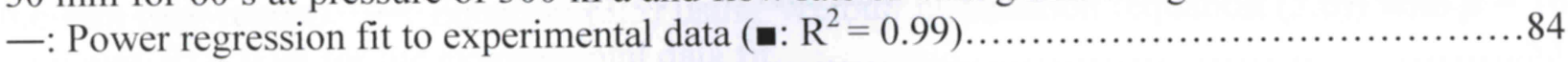

Figure 4.4: Average $E^{*}$ vs. $\sin (\alpha)$ (corresponding to data in Table B-9b). Best fit velocity exponent $k=1.59$. Erosion of Borofloat glass by $25 \mu \mathrm{m}$ aluminum oxide particles blasted at $d=$ $30 \mathrm{~mm}$ for $60 \mathrm{~s}$ at pressure of $300 \mathrm{kPa}$ and flow rate of $3.10 \mathrm{~g} / \mathrm{min}$ using $0.76 \mathrm{~mm}$ round nozzle.

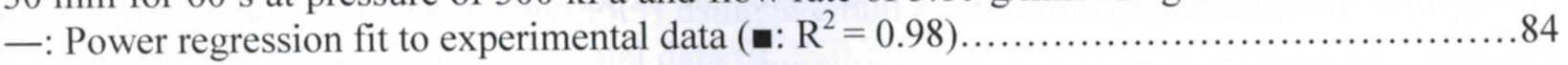

Figure 4.5: Calculated $v^{*}\left(r^{*}\right)$ vs. $r^{*}$ (corresponding to data in Table B-1a and particle spatial distribution in Figure 3.4) using $k=1.57$ (Figure 4.2) for $10 \mu \mathrm{m}$ angular aluminum oxide blasted at $d=30 \mathrm{~mm}$ at pressure of $200 \mathrm{kPa}$ and flow rate of $3.97 \mathrm{~g} / \mathrm{min}$ using $0.76 \mathrm{~mm}$ round nozzle. $\ldots$ : Linear regression fit to calculated data $\left(\bullet: \mathrm{R}^{2}=0.75\right)$.

Figure 4.6: Calculated $v^{*}\left(r^{*}\right)$ vs. $r^{*}$ (corresponding to data in Table B-2 and particle spatial distribution in Figure 3.5) using $k=1.43$ (from [25]) for $25 \mu \mathrm{m}$ angular aluminum oxide blasted at $d=30 \mathrm{~mm}$ at pressure of $200 \mathrm{kPa}$ and flow rate of $2.83 \mathrm{~g} / \mathrm{min}$ using $0.76 \mathrm{~mm}$ round nozzle. -: Linear regression fit to calculated data $\left(\bullet: \mathrm{R}^{2}=0.88\right)$

Figure 4.7: Calculated $v^{*}\left(r^{*}\right)$ vs. $r^{*}$ (corresponding to data in Table B-3 and particle spatial distribution in Figure 3.6) for $50 \mu \mathrm{m}$ silica glass beads blasted at $d=20 \mathrm{~mm}$ at pressure of 200 $\mathrm{kPa}$ and flow rate of $1.17 \mathrm{~g} / \mathrm{min}$ using $0.76 \mathrm{~mm}$ round nozzle. - : Linear regression fit to calculated data $\left(\bullet: k=1.6, \mathrm{R}^{2}=0.92\right)$. ----: Linear regression fit to calculated data $\left(\square: k=2.7, \mathrm{R}^{2}\right.$ $=0.88$ ). 88

Figure 4.8: Calculated $v^{*}\left(r^{*}\right)$ vs. $r^{*}$ (corresponding to data in Table B-4 and particle spatial distribution in Figure 3.7) using $k=1.43$ (from [25]) for $25 \mu \mathrm{m}$ angular aluminum oxide blasted at $d=10 \mathrm{~mm}$ at pressure of $200 \mathrm{kPa}$ and flow rate of $0.36 \mathrm{~g} / \mathrm{min}$ using $0.46 \mathrm{~mm}$ H.P. round nozzle. —: Polynomial regression fit to calculated data $\left(\triangleleft: \mathrm{R}^{2}=0.85\right) \ldots \ldots \ldots \ldots \ldots \ldots \ldots \ldots \ldots \ldots . .88$

Figure 4.9: Calculated $v^{*}\left(r^{*}\right)$ vs. $r^{*}$ (corresponding to data in Table B-5 and particle spatial distribution in Figure 3.8) using $k=1.43$ (from [25]) for $25 \mu \mathrm{m}$ angular aluminum oxide blasted at $d=30 \mathrm{~mm}$ at pressure of $200 \mathrm{kPa}$ and flow rate of $4.75 \mathrm{~g} / \mathrm{min}$ using $1.5 \mathrm{~mm}$ H.P. round nozzle. - : Polynomial regression fit to calculated data $\left(\bullet: \mathrm{R}^{2}=0.95\right)$. 
Figure 4.10: Calculated $v^{*}\left(r^{*}\right)$ vs. $r^{*}$ (corresponding to data in Table B-6 and particle spatial distribution in Figure 3.9) for $50 \mu \mathrm{m}$ silica glass beads blasted at $d=10 \mathrm{~mm}$ at pressure of 200 $\mathrm{kPa}$ and flow rate of $0.23 \mathrm{~g} / \mathrm{min}$ using $0.46 \mathrm{~mm}$ H.P. round nozzle. - Polynomial regression fit to calculated data $\left(\bullet: k=1.6, \mathrm{R}^{2}=0.98\right)$. ----: Polynomial regression fit to calculated data $(\square: k=$ $\left.2.7, \mathrm{R}^{2}=0.96\right)$

Figure 4.11: Calculated $v^{*}\left(r^{*}\right)$ vs. $r^{*}$ (corresponding to data in Table B-7 and particle spatial distribution in Figure 3.10) for $50 \mu \mathrm{m}$ silica glass beads blasted at $d=20 \mathrm{~mm}$ at pressure of 200 $\mathrm{kPa}$ and flow rate of $1.31 \mathrm{~g} / \mathrm{min}$ using $1.5 \mathrm{~mm}$ H.P. round nozzle. - Polynomial regression fit to calculated data $\left(\bullet: k=1.6, \mathrm{R}^{2}=0.89\right)$. ----: Polynomial regression fit to calculated data $(\square: k=$ $\left.2.7, \mathrm{R}^{2}=0.90\right)$

Figure 4.12: Calculated $v^{*}\left(r^{*}\right)$ vs. $r^{*}$ (corresponding to data in Table B-8a and particle spatial distribution in Figure 3.11) using $k=1.44$ (Figure 4.3) for $10 \mu \mathrm{m}$ angular aluminum oxide blasted at $d=30 \mathrm{~mm}$ at pressure of $300 \mathrm{kPa}$ and flow rate of $4.20 \mathrm{~g} / \mathrm{min}$ using $0.76 \mathrm{~mm}$ round nozzle. - - Linear regression fit to calculated data $\left(\bullet: \mathrm{R}^{2}=0.92\right)$ .90

Figure 4.13: Calculated $v^{*}\left(r^{*}\right)$ vs. $r^{*}$ (corresponding to data in Table B-9a and particle spatial distribution in Figure 3.12) using $k=1.59$ (Figure 4.4) for $25 \mu \mathrm{m}$ angular aluminum oxide blasted at $d=30 \mathrm{~mm}$ at pressure of $300 \mathrm{kPa}$ and flow rate of $3.10 \mathrm{~g} / \mathrm{min}$ using $0.76 \mathrm{~mm}$ round

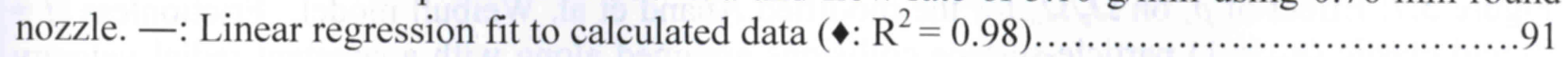

Figure 4.14: Calculated $v^{*}\left(r^{*}\right)$ vs. $r^{*}$ (corresponding to data in Table B-10 and particle spatial distribution in Figure 3.13) for $50 \mu \mathrm{m}$ silica glass beads blasted at $d=20 \mathrm{~mm}$ at pressure of 300 $\mathrm{kPa}$ and flow rate of $1.34 \mathrm{~g} / \mathrm{min}$ using $0.76 \mathrm{~mm}$ round nozzle. - : Linear regression fit to calculated data $\left(\bullet: k=1.6, \mathrm{R}^{2}=0.98\right)$. ----: Linear regression fit to calculated data $\left(\square: k=2.7, \mathrm{R}^{2}\right.$ $=0.97)$

Figure 4.15: Calculated $v^{*}\left(r^{*}\right)$ vs. $r^{*}$ (corresponding to data in Table B-11 and particle spatial distribution in Figure 3.6) for $50 \mu \mathrm{m}$ silica glass beads blasted at $d=20 \mathrm{~mm}$ at pressure of 200 $\mathrm{kPa}$ and flow rate of $0.59 \mathrm{~g} / \mathrm{min}$ using $0.76 \mathrm{~mm}$ round nozzle. - : Linear regression fit to calculated data $\left(\bullet: k=1.6, \mathrm{R}^{2}=0.99\right)$. ----: Linear regression fit to calculated data $\left(\square: k=2.7, \mathrm{R}^{2}\right.$ $=0.99)$.

Figure 5.1: Side and radial plane views of a divergent particle jet impacting the target surface.

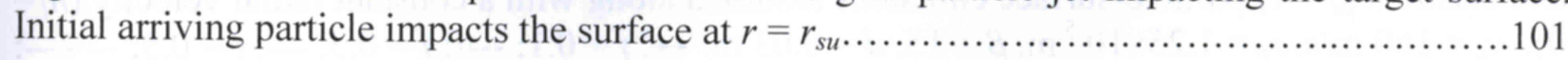

Figure 5.2: Collision between an arriving and a departing particle at $r=r_{\text {coll,su. }}$. The left-hand side of the diagram shows an imaginary departing jet plume for $e_{p s}=1, f=0$, for all $r_{s u}$ (broken lines), and for $e_{p s} \neq 1, f \neq 0$, for a specific $r_{s u}$ (solid lines). The right-hand side of the diagram shows the arriving jet plume (solid bold lines) along with the real departing jet plume for $e_{p s}=1$, $f=0$, for all $r_{s u}$ (broken lines), and for $e_{p s} \neq 1, f \neq 0$, for a specific $r_{s u}$ (solid lines), both extended from the imaginary side. The radial plane view shows the collision cross section..... 106 
Figure 5.3: Effect of $\rho_{s}$ on $\Omega_{z} / \Omega_{a}$ for the modified Anand et al. Weibull model. Frictionless $(f=$ $0)$ and elastic $\left(e_{p s}=1\right)$ particle-surface collisions assumed along with a constant radial velocity $\left(h_{l}=0\right) .-: v_{a}=160 \mathrm{~m} / \mathrm{s}, r_{p}=1.25 \times 10^{-5} \mathrm{~m}, \beta=15, d=0.03 \mathrm{~m}$ (Base Case) (Table 5.1)....

Figure 5.4: Effect of $\rho_{s}$ on $\Omega_{z} / \Omega_{a}$ for the modified Anand et al. Weibull model. Frictionless $(f=$ $0)$ and elastic $\left(e_{p s}=1\right)$ particle-surface collisions assumed along with a constant radial velocity $\left(h_{l}=0\right) . \quad r_{p}=1.25 \times 10^{-5} \mathrm{~m}, d=0.03 \mathrm{~m} .-v_{a}=100 \mathrm{~m} / \mathrm{s}, \beta=13 ;----: 200 \mathrm{~m} / \mathrm{s}, \beta=17$ (Table 5.1).

Figure 5.5: Effect of $\rho_{s}$ on $\Omega_{z} / \Omega_{a}$ for the modified Anand et al. Weibull model. Frictionless $(f=$ $0)$ and elastic $\left(e_{p s}=1\right)$ particle-surface collisions assumed along with a constant radial velocity $\left(h_{l}=0\right) . v_{a}=160 \mathrm{~m} / \mathrm{s}, d=0.03 \mathrm{~m} .-r_{p}=5 \times 10^{-6} \mathrm{~m}, \beta=16$ (Table 5.1).

Figure 5.6: Effect of $\rho_{s}$ on $\Omega_{z} / \Omega_{a}$ for the modified Anand et al. Weibull model. Frictionless $(f=$ $0)$ and elastic $\left(e_{p s}=1\right)$ particle-surface collisions assumed along with a constant radial velocity $\left(h_{l}=0\right) . v_{a}=160 \mathrm{~m} / \mathrm{s}, d=0.03 \mathrm{~m} . \quad-: r_{p}=2.5 \times 10^{-5} \mathrm{~m}, \beta=14$ (Table 5.1$)$. .122

Figure 5.7: Effect of $\rho_{s}$ on $\Omega_{z} / \Omega_{a}$ for the modified Anand et al. Weibull model. Frictionless $(f=$ $0)$ and elastic $\left(e_{p s}=1\right)$ particle-surface collisions assumed along with a constant radial velocity $\left(h_{l}=0\right) . \quad v_{a}=160 \mathrm{~m} / \mathrm{s}, r_{p}=1.25 \times 10^{-5} \mathrm{~m}, \beta=15$. - $: d=0.015 \mathrm{~m} ;----: d=0.06 \mathrm{~m}$ (Table $5.1)$.

Figure 5.8: Effect of $\rho_{s}$ on $\Omega_{z} / \Omega_{a}$ for the modified Anand et al. Weibull model. Frictionless $(f=$ $0)$ and elastic $\left(e_{p s}=1\right)$ particle-surface collisions assumed. $v_{a}=160 \mathrm{~m} / \mathrm{s}, r_{p}=1.25 \times 10^{-5} \mathrm{~m}, \beta=15$. $-: d=0.015 \mathrm{~m}, h_{l}=-5.33 \times 10^{4} \mathrm{~s}^{-1} ;----: d=0.03 \mathrm{~m}, h_{l}=-2.67 \times 10^{4} \mathrm{~s}^{-1}$ (Table 5.1). 123

Figure 5.9: Effect of $\rho_{s}$ on $\Omega_{z} / \Omega_{a}$ for the modified Anand et al. Weibull model. Frictionless $(f=$ $0)$ and inelastic $\left(e_{p s} \neq 1\right)$ particle-surface collisions assumed along with a constant radial velocity $\left(h_{l}=0\right) . \quad v_{a}=160 \mathrm{~m} / \mathrm{s}, r_{p}=1.25 \times 10^{-5} \mathrm{~m}, \beta=15, d=0.03 \mathrm{~m} .-: e_{p s}=0.8 ;----: e_{p s}=0.5 ; \ldots . . \cdot e_{p s}=$ $0.3 ;---: e_{p s}=0.1($ Table 5.1$)$

Figure 5.10: Effect of $\rho_{s}$ on $\Omega_{z} / \Omega_{a}$ for the modified Anand et al. Weibull model. Frictional $(f \neq 0)$ and elastic $\left(e_{p s}=1\right)$ particle-surface collisions assumed along with a constant radial velocity $\left(h_{l}=\right.$ $0) . v_{a}=160 \mathrm{~m} / \mathrm{s}, r_{p}=1.25 \times 10^{-5} \mathrm{~m}, \beta=15, d=0.03 \mathrm{~m} .-$ : $f=0.1 ;---\cdot f=0.3 ; \ldots . .: f=0.5 ;---$ : $f=0.8$ (Table 5.1) 


\section{List of Appendices}

Appendix A Measured $M\left(R^{*}\right) / M(0)$ Values, used in Obtaining the Particle Spatial Distribution across the Jet, along with measured Flow Rates (Corresponding

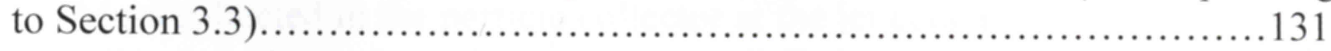

Appendix B Measured $\delta\left(r^{*}\right) / \delta(0)$ and Calculated $v^{*}\left(r^{*}\right)$ Values, along with Measured $k$ Values (Selected Cases) used in Obtaining $v^{*}\left(r^{*}\right)$ (Corresponding to Section $4.3)$. .144 


\section{Nomenclature}

d

$d_{d, s u}$

$d^{*}$

$e_{p p}, e_{p s}$

E

$E^{*}$

$f$

$h_{l}$

$H_{l}, H_{p l}, H_{p 2}$

$i_{\max }$

$k$

$l$

$L$

$L_{\alpha}$

$L^{*}$

$m_{p}$

$M$
Standoff distance (nozzle tip-to-target distance)

"Standoff distance" for the departing trajectory (divergent jet)

Dimensionless standoff distance; the number of particles leaving the nozzle in the time it takes an arriving particle to travel a distance $d[10,12]$

Coefficients of restitution for particle-particle and particle-surface collisions

Erosion rate (mass of target material removed per mass of particles incident on the target)

Normalized erosion rate with respect to value at normal impact

Friction coefficient at surface

Slope that defines the linear velocity distribution decrease with $r$, measured from the jet center $(\mathrm{m} / \mathrm{m} \cdot \mathrm{s})$

Constants for the linear and polynomial regression fits for normalized velocity distribution

Defines amount of tubes of thickness $L_{\alpha}$ in the jet

Velocity exponent

Longer side of inner opening of rectangular nozzle

Mean distance between particles (for $\alpha=90^{\circ}$ ) in the radial direction (for a non-divergent jet) $[10,12]$

Mean distance between particles (for $\alpha \neq 90^{\circ}$ ) in the radial direction (for a non-divergent jet)

Dimensionless distance (in the radial plane) between incident particles $[10,12]$

Particle mass

Total erodent mass 
$\dot{M}_{\text {crit }}$

$M(0)$

$M(R)$

$M\left(R^{*}\right)$

$M(X), M(Y)$

$M\left(X^{*}\right), M\left(Y^{*}\right)$

$d N_{a}, d N_{d}$

$d N_{a c}, d N_{d c}$

$d N_{a, s u}$

$d N_{d, s u}$

$d N_{t o t, s u}$

$N_{t o t, s u}$
Mass flow rate through the nozzle $(\mathrm{kg} / \mathrm{s})$

Critical mass flow rate $(\mathrm{kg} / \mathrm{s})$

Mass collected in the particle collector at the jet center

Mass collected in the particle collector at a specific position $r=R$ (round nozzles)

Mass collected in the particle collector at a specific non-dimensional position $r^{*}=R^{*}$ (round nozzles)

Mass collected in the particle collector at a specific position along the center line $x=X$ or $y=Y$, respectively (rectangular nozzles)

Mass collected in the particle collector at a specific non-dimensional position along the center line $x^{*}=X^{*}$ or $y^{*}=Y^{*}$, respectively (rectangular nozzles)

Change in the number of arriving and departing particles per unit cross sectional area of an $L_{\alpha}$ thick tube at $r_{i}$, spanning the distance $\mathrm{d} u_{a}$ and $\mathrm{d} u_{d}$, respectively

Change in the number of arriving and departing particles per unit cross sectional area of the central cylinder at $r=0$, spanning the distance $\mathrm{d} u_{a}$ and $\mathrm{d} u_{d}$, respectively

Change in the number of arriving particles per unit cross sectional area at the cross section $d u_{a}$ (i.e., at any $r_{\text {coll,su }}$ ) after leaving the nozzle (divergent jet)

Change in the number of departing particles per unit cross sectional area at the cross section $d u_{d}$ (i.e., at any $r_{\text {coll,su}}$ ) after impacting the surface at a particular $r_{s u}$ (divergent jet)

Change in the total number of departing and arriving particles per unit cross sectional area at the cross section $d u_{d}$ or $d u_{a}$ (i.e., at any $r_{\text {coll,su }}$ ), (divergent jet)

Total number of departing and arriving particles per unit collision cross sectional area for any $r_{\text {coll,su}}$, covering all the possible collision zones 
$p_{0}$

$p_{\text {coll }}(r)$

$p_{\text {coll }}\left(r_{\text {coll, su }}\right)$

$p_{i}$

$P_{2}{ }^{W}(r), P_{3}{ }^{W}(r)$

Proportion of the total jet cross sectional area taken by a cylinder of radius $L_{\alpha} / 2$ located at $r_{0}$

Probability that a single arriving particle undergoes a collision with any single departing particle at $r$ at the cross section $d u_{d}$ or $d u_{a}$

Probability that a single arriving particle undergoes a collision with any single departing particle at $r_{s u}$ at the cross section $d u_{d}$, or equivalently, $d u_{a}$ (divergent jet)

Proportion of the total jet cross sectional area taken by a tube of thickness $L_{\alpha}$ located at $r_{i}$

Weibull distribution with shape parameters of 2, 3, respectively (round nozzles)

$P_{2}{ }^{W}(x), P_{3}{ }^{W}(x), P_{4}{ }^{W}(x)$ Weibull distribution as a function of $x$ with shape parameters of $2,3,4$, respectively (rectangular nozzles)

$P_{2}^{W}(y)$

Weibull distribution as a function of $y$ with shape parameter of 2 (rectangular nozzles)

$P_{a}(r)$

Probability distribution (or pdf) for the arriving jet (divergent jet)

$P_{a, d e n} P_{d, d e n}$

$P_{a c, d e n} P_{d c, d e n}$

$P_{\text {coll }}(0)$

Probability density per unit volume of finding an arriving or a departing particle at $r_{i}$, respectively

Probability density per unit volume of finding an arriving or a departing particle at $r_{0}$, respectively

Total probability of collision between an arriving and a departing particle at $r_{0}$

$P_{\text {coll }}\left(r_{i}\right)$

Total probability of collision between an arriving and a departing particle at $r_{i}$

$P_{\text {coll }}\left(r_{\text {coll }, \text { su }}\right)$

Total probability of collision for an arriving particle and a departing particle at $r_{\text {coll,su }}$ (divergent jet)

$P_{d}(r)$

Probability distribution (or pdf) for the departing jet (divergent jet)

$P_{\text {surv }}(0)$

Total probability of a particle surviving through the distance $z_{\text {mean }}$ and reaching the surface at a cylinder of radius $L_{\alpha} / 2$ located at $r_{0}$ 


$$
P_{\text {surv }}\left(r_{i}\right)
$$

$P_{\text {surv }}\left(r_{\text {coll,su }}\right)=$

$P_{\text {surv }}\left(r_{\text {surv }, \text { su }}\right)$

$$
P_{\text {surv }}\left(r_{\text {coll, su }}^{-}\right)
$$

$P_{\text {surv }}\left(r_{\text {coll }}^{-}\left(r_{\text {su }}^{-}\right)\right)$

$P(r) d r$

$P\left(r^{*}\right) d r^{*}$

$r$

$r^{*}=r / d$

$r_{0}$

$r_{c}$

$r_{c}{ }^{*}=r_{c} / d$

$r_{\text {coll,su }}$

$r_{\text {coll,su }}$

$r_{\text {coll }}^{-}\left(r_{\text {su }}^{-}\right)$

$r_{i}$

$r_{n}$
Total probability of a particle surviving through the distance $z_{\text {mean }}$ and reaching the surface at a particular tube at radius $r_{i}$

Total probability of a particle surviving a collision at $r_{\text {coll,su }}$, or equivalently, reaching the surface at $r_{\text {surv,su }}$ (divergent jet)

Mean probability that an arriving particle traveling on a trajectory defined by the angle $\phi_{\text {coll }, s u}^{-}$does not collide at $r_{\text {coll,su }}^{-}$, at some distance $\overline{u_{d}}$ above the surface, and thus survives to the surface at $r_{\text {surv,su }}^{-}$(divergent jet)

Ratio of the total number of particles surviving to the entire surface through all the rebound heights (collision cross sections) over which collisions can occur to the total number of particles launched from the nozzle (divergent jet)

Probability of finding a particle between $r$ and $r+d r$

Probability of finding a particle between $r^{*}$ and $r^{*}+d r^{*}$

Radial position in the jet, measured from the nozzle axis (jet center)

Normalized radial coordinate

Location of cylinder of radius of $L_{\alpha} / 2$ at $r=0$

Radius of the catcher tube

Non-dimensional collection cylinder radius

Radial position at which a collision between an arriving and a departing particle (after impacting the surface at $r_{s u}$ ) occurs (divergent jet)

Mean $r_{\text {coll,su }}$ at which a collision "typically" occurs (divergent jet)

Mean $r_{\text {coll,su }}$ evaluated at a mean $r_{s u}, r_{s u}^{-}$(divergent jet)

Location of an $i^{\text {th }}$ tube of thickness $L_{\alpha}$, measured from jet center

Nozzle radius 
$r_{\max }$

$r_{p}$

$r_{p}{ }^{*}, r_{n}{ }^{*}$

$r_{s u}$

$r_{s u}$

$r_{\text {surv }, s u}$

$r_{\text {surv }, \text { su }}$

$R$

$R^{*}=R / d$

$s$

$d t$
Radial position at which the probability of finding a particle arriving between $r$ and $r+\mathrm{d} r$ at the surface, $P(r) d r$, becomes exceedingly small (i.e., $1 \%)$ (divergent jet)

\section{Particles radius}

Dimensionless particle and nozzle radii; the number of particles leaving the nozzle in the time it takes an arriving particle to travel a distance equal to $r_{p}$ or $r_{n}$, respectively $[10,12]$

Arbitrary radial position at which an initial arriving particle impacts the target surface (divergent jet)

Radial position at the surface that a rebounding particle departs from, traveling on a collision trajectory defined by the angle $\psi\left(r_{s u}^{-}\right)$, so that it intersects a mean arrival trajectory at radial position $r_{\text {coll }}^{-}\left(r_{\text {su }}^{-}\right)$and at a mean height of $z\left(r_{s u}^{-}\right) / 2$ above the surface (divergent jet)

Radial position at the surface which an arriving particle reaches if no collision occurs at $r_{\text {coll,su }}$, at some distance $u_{d}$ above the surface (departing jet)

Mean $r_{\text {surv,su }}$ which an arriving particle reaches if no collision occurs at $r_{\text {coll }, s u}^{-}$, at some distance $\overrightarrow{u_{d}}$ above the surface (divergent jet)

Distance from the jet center to the center of the collection cylinder (round nozzles)

Non-dimensional distance from the jet center to the center of the collection cylinder (round nozzles)

Velocity at the profile surface in the direction of the unit normal on the surface

The infinitesimal time it takes for the surface at point of impact to reach a depth $\delta$ 
$u_{a}$

$u_{a}$

$u_{d}$

$u_{d}$

$\bar{u}_{a}, \bar{u}_{d}$

$|v|$

$v_{a}$

$v_{a n}, v_{a t}$

$v_{a}\left(r_{s u}\right)=v_{a, s u}$

$v_{a n, s u}, v_{a t, s u}$

$v_{a}\left(r_{c o l l, s u}\right)$

Distance traveled by an arriving particle until it intersects a departing particle trajectory on a collision cross section of thickness $d u_{a}$, measured along the arrival trajectory

Distance traveled by an arriving particle, on a collision trajectory defined by the angle $\phi_{\text {coll }, s u}=\tan ^{-1}\left(r_{\text {coll }, s u} / u_{a}\right)$, after leaving the nozzle, measured perpendicular to the surface, until it intersects a departing particle trajectory on the infinitesimal cross section of thickness $d u_{a}$, at a certain $r_{\text {coll }, s u}$ (divergent jet)

Distance traveled by a departing particle until it intersects an incident particle trajectory on a collision cross section of thickness $d u_{d}$, measured along the arrival trajectory

Distance traveled by a departing particle on a collision trajectory defined by the angle $\psi_{s u}$, after impacting the surface at a particular ring at $r_{s u}$, measured perpendicular to the surface, until it intersects an incident particle trajectory on the infinitesimal cross section of thickness $d u_{d}$ at a certain $r_{\text {coll, su }}$ (divergent jet)

Mean $u_{a}, u_{d}$ at which a collision "typically" occurs, respectively (divergent jet)

Magnitude of the particle impact velocity at a certain point on the profile surface

Incident/arrival particle velocity

Normal and tangential arrival velocity components at the surface, respectively

Velocity of an initial arriving particle at a particular point $r=r_{s u}$ on the surface (divergent jet)

Normal and tangential arrival particle velocity components at a particular point $r_{s u}$ on the surface, respectively (divergent jet)

Velocity of an arriving particle at $r_{\text {coll, su }}$ at some distance $u_{a}$ from the nozzle (divergent jet) 


$$
\begin{aligned}
& v_{d n}, v_{d t} \\
& v_{d, s u} \\
& v_{d n, s u}, v_{d t, s u} \\
& v(r) \\
& v^{*}(r) \\
& v^{*}\left(r^{*}\right) \\
& w \\
& x, y \\
& x^{*}=x / d, y^{*}=y / d \\
& X, Y \\
& X^{*}=X / d, Y^{*}=Y / d \\
& z_{d, \text { mean }} \\
& z_{\text {mean }}=z_{a, \text { mean }} \\
& z\left(r_{s u}\right)=z_{s u} \\
& z\left(r_{s u}^{-}\right) \\
& \alpha
\end{aligned}
$$


$\beta=\beta_{a}$

$\beta_{d, s u}$

$\gamma$

$\delta(r)$

$\delta\left(r^{*}\right)$

$\theta$

$2 \theta_{c}$

$\lambda$

$\mu_{t}$

$\rho_{p}$

$\rho_{s}$

$\rho_{\text {sub }}$

$\sigma$

$v$

$\phi$

$\phi_{\max }$

$\phi_{c o l l, s u}=\phi_{\text {surv }, s u}$
Focus coefficient for the arriving jet (defines the jet focus) [1,15-17]

Focus coefficient for the departing trajectory (divergent jet)

Scatter angle

Profile depth as a function of $r$, measured from the jet center

Profile depth as a function of $r^{*}$, measured from the jet center

Angular position in the radial plane (measured c.c.w. from $r$-axis)

Angular spread of collection cylinder at a particular location $r=R$

Jet divergence angle

Critical impulse ratio

Particle mass density

Dimensionless stream density (ratio of the total volume of particles in the jet to the volume of the jet itself) $[10,12]$

Density of the substrate (i.e., target)

Single particle collision cross section

Angle between the incident velocity vector $v$ and the unit normal on the profile surface

Angle between the nozzle axis and an arriving particle trajectory

Angle defining the trajectory of an arriving particle reaching $r_{\max }$ at the surface, measured from the nozzle axis (divergent jet)

Angle defining the trajectory of an arriving particle reaching $r_{\text {coll,su }}$, or equivalently $r_{\text {surv,su }}$ (if no collision occurs), measured from the nozzle axis (divergent jet) 
$\phi_{\text {coll }, \text { su }}^{-}$

$\phi\left(r_{s u}\right)=\phi_{s u}$

$\psi$

$\psi\left(r_{s u}\right)=\psi_{s u}$

$\psi\left(r_{s u}^{-}\right)$

$\Phi(0)$

$\Phi(r)$

$\Phi^{*}\left(r^{*}\right)$

$\Phi(R)$

$\Phi\left(R^{*}\right)$

$\Omega_{a}$

$\Omega_{a(\text { crit })}$

$\Omega_{d}$

$\Omega_{z}$
Mean angle defining the trajectory of an arriving particle reaching

$r_{\text {coll,su }}^{-}$or equivalently $r_{\text {surv,su }}^{-}$, measured from the nozzle axis (divergent jet)

Angle defining the trajectory of an initial arriving particle reaching $r_{s u}$ at the surface, measured from the nozzle axis (divergent jet)

Angle between the target surface and the departing trajectory

Angle between the target surface and the departing particle trajectory at a particular $r_{s u}$ (divergent jet)

$\psi_{s u}$ evaluated at a mean $r_{s u}, \overline{r_{s u}}$ (divergent jet)

Particle mass flux at $r=0$

Particle mass flux (mass of particles per unit time passing through a unit ring area at a given $r$ )

Non-dimensional normalized mass flux (with respect to jet center)

Particle mass flux at a specific catcher tube position $r=R$

Particle mass flux at a specific non-dimensionalized catcher tube position $r^{*}=R^{*}$

Particle nozzle launch frequency (particles/s)

Estimate of the required maximum critical particle launch frequency (particles/s) for a specified level of particle interference

Frequency of particles departing from the surface after colliding with it (particles/s)

Frequency of particles surviving to the surface through a distance $z$ (through all the possible collision zones) (particles/s) 


\section{Chapter 1 Introduction}

\subsection{Background and Critical Literature Review}

\subsubsection{Particle Interference Effects in Erosion Testing}

In solid particle erosion testing, where particles are accelerated in a gas jet in a nozzle before arriving to a target, the effect of varying particle flux (the mass of particles launched from the nozzle per unit area per second) has been largely ignored, up until recently [1]. It was generally believed that the erosion rate, i.e., the ratio of the target material mass loss to the mass of used particles, did not depend on the particle flux because it was assumed that all particles traveled to the target with the same velocity and impact angle [1]. However, experiments have shown that the erosion rate does in fact depend on the incident particle flux, especially at normal incidence and at high incident particle fluxes [1-9]. This dependency originates from the fact that incident particles can collide with ones that rebound from the target surface (the so-called "particle interference effect"), thereby affecting the distribution of erosive energy of the particles reaching the surface.

Shipway and Hutchings [1] studied the dependence of erosion on varying particle flux in detail. For this work, spherical glass particles were used, with a size range of 63-750 $\mu \mathrm{m}$ (mean diameter). The velocity was measured with an opto-electronic flight timer which used infra-red emitters and detectors attached to the nozzle tip ends. In addition, scar radius experiments were performed at different particle fluxes by blasting particles from a $4.72 \mathrm{~mm}$ inner diameter nozzle onto flat aluminum targets covered with a thin dye at normal incidence, with a $20 \mathrm{~mm}$ standoff distance (i.e., nozzle tip-to-target distance). The scar radius was measured as the effective area of the region where a critical dose of launched particles was just high enough to remove the thin film of die from the aluminum targets. A number of important observations were made in this work. Firstly, it was concluded that the particle velocity was independent of the particle flux because mean inter-particle spacing within the nozzle was calculated to be high enough so that inter-particle collisions in the radial plane could be ignored. Secondly, it was shown that at low fluxes, the scar radius increased linearly with the logarithm of mass of erodent used. However, when the particle flux was increased to relatively high levels, for otherwise identical conditions, 
the relationship between scar radius and the logarithm of erodent mass used began to take the form of an increasing exponential. This departure from a linear behaviour was attributed to increased particle interference beyond a certain critical particle flux, which also explains why the erosion rate decreases beyond this critical particle flux.

It was also found that the interference effect (i.e., the nonlinear behaviour) was more pronounced as particle size increased. It was postulated that this was due to a higher probability of collision for larger particle sizes near the surface and increased particle interactions with the rim of the nozzle, which could increase the jet divergence and therefore a more dramatic increase in the scar radius was observed. Although for smaller particles, these effects should have been much less significant, a "nonlinear" behaviour was also noticed. It was postulated that this behaviour might have been caused by other factors, such as aerodynamic effects originating from the gas jet, which could increase the tendency for particles to acquire a higher tangential velocity and therefore a more dramatic increase in the scar radius for smaller particles was also observed.

A decrease in erosion rate beyond a certain critical flux due to the particle interference effect has been reported by other researchers [2-6]. Chevallier and Vannes [2] studied the effect of a divergent (i.e., cone-shaped) erosive jet on a sheet metal surface upon impact. They discovered that the highest percentage of particles arrived to the target near the jet center and that these particles interfered with ones arriving at the periphery of the jet. They also postulated that the erosion rate at the surface decreased because an increase in flux introduced these collisions, which created a particle velocity gradient across the jet and varied the particle scattering directions. However, these findings were only explained qualitatively; i.e., without quantifying the flux effects pertinent to this behaviour.

In't Veld and Slikkerveer [3] studied the effect of varying flux for a previously defined interference model by Anand et al. [9], which will be discussed in the following section. They introduced a correction factor for the incident particle velocity, assuming that it varied across the jet, and the surface impact area to model a divergent as opposed to a cylindrically focused jet. Although they used relatively small particles $(23.5-29 \mu \mathrm{m})$ and large inner diameter nozzles (1.5$12 \mathrm{~mm}$ ), they found that an increase in the flux caused a significant drop in erosion and assumed that an increase in velocity caused a decrease in the effective collision length (the mean height above the surface where particles collide, i.e., the height of the collision zone), implying a higher flux gradient. This, however, contradicts the findings of [1] where increases in particle flux for 
highly sparse jets (i.e., where the mean distance between particles is large, thus the probability of particle interference is low) did not significantly affect the erosion rate. In reality, the flux had an effect on interference because the Anand et al. [9] interference model does not account for particle spacing and can only be used for low flux conditions. Thus, for higher fluxes, the model predicts a rapid decrease in the erosion rate. In't Veld and Slikkerveer also concluded that the velocity was one of the foremost predictors of flux effects because they found a high correlation between velocity and the effective collision length. However, decreases/increases in velocity play a more significant role in increasing/decreasing the probability of particle collision by decreasing/increasing the particle spacing in the jet, which is the more significant predictor of reduced erosion rates for the substrate $[1,4,10]$. Therefore, it could be said that velocity contributes to the interference effect, but it is only one of many factors.

The work done by Oka et al. [4] in a sand erosion testing facility also supports the idea that particle interference is an important parameter affecting erosion. The effect of particle impact velocity, impact angle, and flux on erosion rate were examined. It was discovered that the relative distance between particles defined in Ref. [1], was a high predictor of decreases in erosion rate beyond some critical point. This supports the findings of Ref. [1], since a highly sparse jet (i.e., where the distance between particles is high) is not affected by flux variations because the probability of particle collisions is low. The researchers of Ref. [5] also supported the fact that the erosion rate at the surface decreased beyond a certain critical particle size, since larger particles decreased the relative distance between particles thereby increasing the probability of collision.

Particle interference may also contribute to the explanation of why, in some cases, for brittle substrates, where erosion by fracture occurs, the optimum erosion rate does not occur at normal incidence where the highest transfer of erosive energy to the surface occurs, but at some intermediate angle [6,7]. At normal incidence, more of the particles interfere with each other than at other intermediate angles because the collision volume at normal incidence spans the whole jet and hence the probability of collision is higher. Hence, the effective erosive energy at the target is reduced at normal incidence. For ductile materials, where erosion by cutting occurs, the relationship between the maximum erosion rate and the impact angle depends more so on the erosion mechanism [6,7]. Since cutting is more efficient at shallow angles than at normal incidence, the maximum erosion rate occurs at some non-normal incident angle. Particle 
interference might contribute to this relationship dependence, but not as significantly as the erosion mechanism.

\subsubsection{Analytical Modeling of Particle Interference}

Most investigators in the past have qualitatively referred to the interference effect as the main or partial reason for reduced erosion rate with increasing particle flux. However, little has been done to quantitatively describe this phenomenon by modeling its behaviour. A few investigators have attempted to construct analytical models that assess these particle interference effects. Andrews and Horsfield [8] derived the probability of two particles colliding in a particle jet, quantified the mean free path for a spherical particle, and found the variation of the relative energy of scattered particles with the energy of the incident particles as a function of the scatter angle. The results were compared to those of a sand erosion test and it was found that theory seemed to agree with experimental findings. In addition, the researchers derived an expression for the collision frequency for low fluxes and concluded that collisions do not reduce the number of particles that arrive to the target surface. They explained that reduction in erosion rate is the result of particle collisions degrading the incident beam by increasing the angular spread and by increasing the variation of velocities and impact angles near the surface. This is consistent with the findings of Ref. [2].

Anand et al. [9] derived the probability of collision between an incident and a rebounding sphere at steady state, and found an exponential dependence of erosion rate on particle mass flux (i.e., the erosion rate decreased exponentially with increasing flux). It was also found that the target was partially protected from the incident beam due to particle interference.

The analytical models of Refs. [8,9] have many limitations. They are single particle collision models; i.e., a given incident particle is allowed to collide with a rebounding one only once, after which it is removed from the jet. Also, it is assumed that all the particles arriving to the surface travel in parallel paths and rebound from the surface with the same average angle, and this angle is a model input (i.e., it must be determined a priori). In addition, these models do not account for radial symmetry, stream density (the ratio of the volume of particles in the jet to the volume of the jet itself, i.e., the fact that there are spaces between particles as they travel to the surface), and particle-to-surface collision energy losses. Moreover, the models assume a 
non-divergent jet and a constant velocity distribution across the jet, do not consider complex rebound dynamics (i.e., 2-dimesional (2D) frictionless elastic inter-particle and particle-surface collisions are assumed), and ignore the effect of the nozzle-to-surface standoff distance.

\subsubsection{Computer Modeling of Particle Interference}

The limitations of the above analytical interference models of Refs. [8,9] led Ciampini et al. [10] to develop a 3-dimensional (3D) computer model to predict the effect of particle interference on the incident erosive power to the surface. The model was written in the computer language $\mathrm{C}++$ and enables the user to visualize the simulation. The model simulated incident divergent jets (with $\lambda$ as the jet divergence angle) of spherical particles of radius $r_{p}$ and mass $m_{p}$, originating from a nozzle of radius $r_{n}$ at an angle of incidence $\alpha$ with a launch frequency $f_{l}$, arriving to the surface with a velocity $v_{a}$, at a standoff distance $d$ (nozzle tip-to-target distance). A coefficient of restitution approach was used to model particle-particle and particle-surface collision energy losses, with $e_{p p}$ and $e_{p s}$ being the respective coefficients of restitutions for each collision case. It was also assumed that particle-particle collisions were frictionless while a friction coefficient $f$ was used to model particle-surface collisions. Aerodynamic effects were assumed to be negligible, hence the particles did not diverge from their original trajectories when traveling from the nozzle to the surface. For divergent jets, particles launched from the nozzle at a distance $r$ from the jet center were assumed to follow the path defined by:

$$
\vartheta(r)=\lambda \frac{r}{r_{n}} \quad 0 \leq r \leq r_{n}
$$

where $\vartheta(r)$ is the angle between the normal to the nozzle and the path of a particle leaving the nozzle at a radius $r$. Further, a uniform velocity distribution was assumed and only one particle was launched at a time from the nozzle (however, the model allows for multiple simultaneous launches).

The simulation is event driven, meaning that time increments are taken from one collision event to another, for all possible collisions (both particle-particle and particle-surface collisions). This allows individual particles to experience collisions multiple times in one simulation. 
Particles arrive to the surface randomly at a particular $\vartheta(r)$ and $r$. Also, data are collected at steady state, i.e., when the number of particles entering and exiting a predefined control volume is approximately constant.

Particle-surface collisions and their respective scattering directions are modeled using simplified Brach analysis [11] developed for collision between a sphere and a flat surface, assuming a singular point of impact. The model accounts for cases of sliding or rolling through the impact during a surface collision and calculates the rebound velocities and their respective directions. For inter-particle collisions, since frictional effects were ignored, only normal velocity components had to be considered in calculating particle scatter velocities and their respective directions.

The model was verified against experimental data from Refs. [1] and [4]. The computer simulations yielded similar result for critical fluxes (fluxes that define the onset of critical interference) defined in [1], for three different spherical particle sizes, for the same set of conditions. Also, when it comes to the relative distance between particles in the radial plane, defined in [1], the results of [4] matched the results of the computer simulation with respect to the relative drops in erosion for denser jets, implying a higher probability for particle collisions and hence interference. The model also supports the general findings of Ref. [5].

The computer model has some limitations originating from its general assumptions. Firstly, inter-particle collisions were assumed to be frictionless. However, this assumption is justified in many cases since most of the impact energy originates from particle-surface interactions and because the rotational energy transfer caused by frictional effects is negligible when compared to the linear energy transfer during particle-particle collisions. Secondly, aerodynamic effects were neglected, an assumption that is valid if the gas velocity driving the particles to the surface is negligible near the surface. Thirdly, the velocity and spatial distribution were assumed constant and uniform across the jet, respectively. In reality, the velocity has a peak at the nozzle center and it is lowest near the nozzle walls, and the spatial distribution is non-uniform across the jet. Fourthly, only round nozzles were considered. Other nozzle shapes yield a different energy availability to the surface and hence a different interference pattern. Additionally, particles were launched one at a time. However, this seems to make no difference when compared to two or more particles being launched simultaneously, for the high flux cases. Finally, surface energy losses were modeled using the coefficient of 
restitution approach as opposed to a material removal mechanism. The simulation is not valid for cases where significant craters form on the surface because that would deviate from the model assumption that the surface remains locally flat after impact. This would yield different rebound velocities and angles, and thus create different interference effects.

A dimensional analysis was performed by Ciampini et al. [12] on all the jet variables previously defined in a follow-up paper. These dimensionless variables will be discussed in more detail in the next chapter. The output of the model was the non-dimensional power available to a pre-defined target surface area or the entire surface (both normalized with respect to the power available from the nozzle), which included the effect of particle interference. It was found, through a sensitivity analysis, that the greatest effect on this power was due to changes in the angle of incidence, followed by the dimensionless particle radius (a ratio of the particle flux times the particle radius to particle velocity), followed by the coefficient of restitution for particle-surface interactions. It was also discovered that interactions between these parameters led to fairly significant changes in power outputs. Individual changes in the dimensionless standoff (a ratio of the particle flux times the standoff to particle velocity), the divergence angle, and the dimensionless nozzle radius (analogous to the dimensionless particle radius) were responsible for the next three greatest effects on the power availability. Finally, it was found that the smallest effect was due to changes in the coefficient of restitution for particle-particle interactions, followed by changes in the friction coefficient. Some of these parameters were further non-dimensionalized by defining a new variable: the stream density (i.e., the ratio of the volume of particles in the jet to the volume of the jet itself). The stream density is a function of dimensionless particle radius, nozzle radius, standoff, and the jet divergence angle.

With this in mind, the investigators of Ref. [12] carried out a parametric study for the most important jet parameters and the following conclusions were reached: (a) When the nozzle radius was 15 times that of the particle radius or greater, the most vital variables affecting interference effects were the angle of incidence, stream density, coefficient of restitution for particle-surface interactions, and for normal incidence only, the standoff distance. The standoff had no effect on interference at oblique incidence because for a particular angle of attack in this range, the collision volume stays constant, regardless of the standoff; (b) At low stream density, the interference effect was negligible and so the dimensionless power available to the target surface area was approximately equal to 1 . An increase in stream density resulted in more 
particle scattering and thus a more sparsely distributed power available to the target surface. These results confirm previous findings on the topic of interference (as in Ref. [1] for instance). In addition, because stream density is a function of many input jet parameters, including velocity and flux, it is a parameter that accounts for all these variables; (c) A drop in the coefficient of restitution for particle-surface collisions resulted in an increase in the power available to the target area and the entire surface. In some cases, the power availabilities were higher than 1 (i.e., exceeded the power available from the nozzle). This results from a decrease in the rebound particle velocity near the surface, which increases the chance for inter-particle collisions, and thus increases the surface impact frequency due to multiple surface interactions (i.e., particles rebound from the surface, collide with incoming particles, and are redirected back towards the surface). It can thus be said that particle interference does not always result in decreases in erosion at the surface, as was seen in Ref. [3], especially for brittle materials. It should be noted that the analytical models in Refs. [8, 9] cannot account for this effect because they are singlecollision models and cannot predict multiple surface impacts; (d) The highest power availabilities to the target and the entire surface were found to occur for low angles of incidence. Also, the scattering effect was greatest for high angles of incidence (due to an increase in the collision volume and thus an increase in particle interference); (e) At normal incidence, the power availability decreased with increasing standoff. For higher standoff, the collision volume is larger, and thus more collisions result in less particles arriving to the surface. This effect was greatest at low stream density; (f) The model cannot easily generalize interference effects for nozzle-to-particle radius ratios of less than 15 . However, it still accurately predicts interference in that range.

Papini et al. [13] and Gomes-Ferreira et al. [14] resumed work in computer modeling of particle interference by studying the effects of the plume shape. In order to simulate scar radius experiments performed by Shipway and Hutchings [1], the model of Refs. [10,12] was modified to include a radial distribution of particles, as opposed to a random one (as defined by equation (1.1)), defined by Shipway and Hutchings [15]:

$$
P(r) d r=\left(\frac{\beta}{d}\right)^{2} r e^{-\left(\frac{\beta}{d}\right) r} d r
$$


where $P(r) d r$ is the probability of finding a particle between $r$ and $r+d r, P(r)$ is the Gamma distribution (or probability density function (pdf)), $d$ is the standoff distance, measured from the nozzle tip to the target surface, $r$ is the distance from the jet center (i.e., the radial distance from the nozzle axis), and the dimensionless parameter, $\beta$, is "the focus coefficient" (Figure 1.1). $\beta$ depends on the internal nozzle roughness, the particle velocity, and the nature of the erodent particles used [15]. A higher $\beta$ implies a more focused jet; i.e., the jet divergence is lower, and a higher standoff distance, $d$, results in a larger scar radius [15]. Equation (1.2) can also be used to obtain the angular distribution of particles [15]:

$$
P(\phi) d \phi=\beta^{2} \frac{\sin \phi}{\cos ^{3}(\phi)} e^{-\beta \tan \phi} d \phi
$$

since $P(\phi) d \phi=P(r) d r$ and $r=d \tan (\phi)$, where $P(\phi) d \phi$ is the probability of finding a particle between $\phi$ and $\phi+d \phi, \phi$ is the angle between the nozzle axis and particle trajectory, and $P(\phi)$ is the Gamma distribution in terms of $\phi$. This assumes that $P(\phi) d \phi$ is independent of $d$, and hence the particles are assumed to travel in straight lines from the nozzle to the target surface. The distribution defined by equation (1.2) and (1.3) assumes that particles emanate from a point source, as opposed to a nozzle of finite size [15].

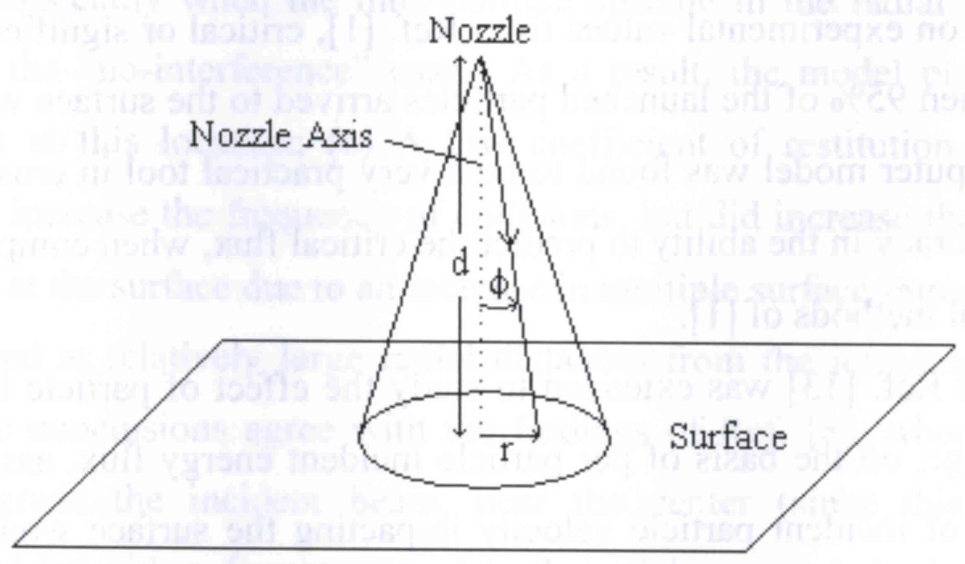

Figure 1.1: Geometric definitions for a plume of particles, emanating from a point source ("nozzle"), and arriving to a surface at a radius $r$.

The model assumed that only the normal component of velocity contributed to coating removal in order to match the assumptions of Ref. [1]. The model simulated the total normal 
particle incident energy per unit impact area at the surface which could be compared to the total normal critical particle incident energy per unit impact area obtained experimentally from [1], and as defined by [15]:

$$
Q_{c r i t}=\frac{M P(r)}{2 \pi r}
$$

where $Q_{\text {crit }}$ is the critical mass of particles needed to remove the dye from the surface per unit area on the target, $M$ is the total erodent mass, and $P(r)$ is obtained from equation (1.2). The results of the simulation matched the experimental results of [1] within $5 \%$ when the scar radius was plotted against the logarithm of the total mass of erodent used, for several flux levels. In addition to this, the computer model was able to accurately predict the critical flux, which determines the onset of significant particle interference. For erosive jets at normal incidence, originating from relatively small nozzles when compared to the standoff distance, the critical launch frequency is a function of the standoff, particle radius, particle velocity, the coefficient of restitution for particle-surface interactions, and the focus coefficient which characterizes jet divergence. Dimensional analysis allowed plots covering a wide range of incident parameters to be generated, from which the critical particle mass flow rate or mass flux could be easily determined. Based on experimental values from Ref. [1], critical or significant interference was defined to occur when $95 \%$ of the launched particles arrived to the surface without undergoing a collision. The computer model was found to be a very practical tool in erosion testing, because of its speed and accuracy in the ability to predict the critical flux, when compared to the long and tedious experimental methods of [1].

The work of Ref. [13] was extended to study the effect of particle interference, beyond the critical flux range, on the basis of per particle incident energy flux, assuming that only the normal component of incident particle velocity impacting the surface would contribute to the energy exchange [14]. The simulations were used to predict the initial surface profile for a brittle eroded surface, as most of the initial material removal in this case can be contributed to the normal velocity component. A dimensional analysis was performed on the incident per particle energy flux and several jet variables already discussed (i.e., standoff, particle mass, focus coefficient, particle radius, launch frequency, average particle velocity, coefficient of restitution 
for particle-surface interactions, and the radial distance from the jet center), for normal incidence. The resulting dimensionless incident energy flux was divided into two parts: the portion that did not undergo particle collisions before reaching the surface and the portion that did. The computer model was simulated for various input jet parameters, and expressions for the incident energy flux due to non-colliding particles, the incident average velocity, the average impact angle, and the spatial distribution of arriving-to-surface particles for particles undergoing collisions, were derived via curve fitting. The last three expressions were then combined to define the dimensionless incident energy flux for particles undergoing collisions, but only for cases where less than $50 \%$ of all the particles in the jet contributed to interference because beyond that, the derived expressions could not accurately predict the simulated data. Hence, this is one limitation of the model. The incident energy flux was found to generally decrease exponentially with increasing radial distance from the jet center. The model was also found to fit the simplified analytical interference model by Anand et al. [9], for the non-colliding incident energy flux portion only, assuming that the erosion rate is proportional to the energy flux for brittle materials.

The results of the study of Ref. [14] can be summarized as follows: (a) In the vicinity of the jet center, most of the incident energy flux was due to particles free of collisions before reaching the target; (b) The incident energy flux noticeably decreased near the jet center, as found in Ref. [2], especially when the inter-particle spacing in the radial jet plane decreased, when compared to the "no-interference" case. As a result, the model predicted significantly lower erosion rates at this location; (c) A low coefficient of restitution for particle-surface interactions did not increase the frequency of collisions, but did increase the total instantaneous particle energy flux at the surface due to an increase in multiple surface impacts; (d) The incident energy flux increased at relatively large radial distances from the jet center for cases of high interference. These conclusions agree with the findings of Ref. [8], where particle collisions were found to degrade the incident beam, near the center (since this model assumed a cylindrically focused jet with a diameter equal to that of the nozzle), by increasing the angular spread and the variation of velocities and impact angles near the surface, and thereby increasing the relative erosion rate at larger radial distances.

The model of Ref. [13,14] was only applied to cases involving normal incidence. Also, as previously mentioned, the model worked only for cases where less than $50 \%$ of the particles 
in the jet underwent collisions. In addition, the model assumed a uniform velocity distribution across the jet. However, as will be shown in Chapter 4, this is not always the case. Finally, the model is assumed to follow the spatial distribution defined by equation (1.2), the Gamma distribution. However, it will be later shown that this is not a generally applicable distribution. As a result, there is a need to accurately model the spatial and velocity distribution in particle jets which can aid in the development of practical analytical models for divergent jets that can realistically predict particle interference and surface erosion rates for a diverse set of conditions. This will be discussed in the following section.

\subsubsection{Study and Modeling of Spatial and Velocity Distribution in Micro-abrasive Jets}

Shipway and Hutchings studied the velocity and the spatial distribution of particles using the gas-blast erosion method, which accelerates particles through the nozzle in a gas jet before they arrive at the target surface [15]. The influence of internal nozzle roughness was examined for a $308 \mathrm{~mm}$ long nozzle with a $4.72 \mathrm{~mm}$ internal diameter. For this work, angular silica and spherical soda-lime glass particles were used, with a size range of $125-150 \mu \mathrm{m}$. The velocity was measured with an opto-electronic flight timer which used infra-red emitters and detectors attached to the end of the nozzle tip. The gas plume shape was examined using the Schlieren optical technique which is sensitive to the initial spatial derivative of the stream density. The final setup formed a dark-field image in order to be able to differentiate the particle jet shape from the background. Scar radius experiments were performed by blasting particles on flat aluminum targets covered with a thin dye at normal incidence. Both the plume and scar radius were photographed before being analyzed. Based on measurements of the scar radius as a function of mass of erodent launched, an analytical model for the spatial particle distribution was derived. The scar radius $r$ was found to be linearly related to the logarithm of the total erodent mass, $M$, which arrives to the surface, implying that the proportion of the total mass striking the target at distances between $r$ and $r+d r$ from the axis of the nozzle, was defined by $P(r) d r$ (equation (1.2)).

The results of Ref. [15] show that the use of rougher nozzles produced a larger scar radius and led to increases in jet divergence (i.e., lower $\beta$ ) for both glass and sand particles. However, this relationship reached a plateau, beyond which the roughness had no effect, and the 
scar radius remained constant. In addition, the spherical glass particles produced a larger scar radius than the sand particles, for the same nozzle roughness, because they had a higher rebound angle when interacting with the nozzle wall before exiting into the jet. The internal nozzle roughness also influenced the particle velocity distribution. The rougher the nozzle, the lower the resulting particle velocity and the higher the velocity spread, resulting in a higher divergence of the particle jet due to more particle interactions inside the nozzle wall before exiting the nozzle tip.

It was also found in Ref. [15] that the nozzle exit velocity of the sand particles was higher than that for glass particles, for two main reasons. Firstly, for the same average size, the sand particles, due to their irregular shape, had a higher drag coefficient than the spherical glass particles. Secondly, the irregularly shaped sand particles experienced shallower rebound angles than the spherical glass particles, due to their ability to rotate upon impact, which leads to higher velocity upon exit from the nozzle tip. This also explains why the exit velocity of the sand particles was found to be less sensitive to nozzle roughness than that of spherical glass particles.

The technique for measuring particle spatial distribution presented in Ref. [15] has some limitations in its applicability. Firstly, the process is very laborious and time consuming. Secondly, $Q_{c r i t}$ strongly depends on the dye thickness. Since it is usually difficult to apply the dye evenly, any irregularities in the dye thickness may cause $Q_{c r i t}$ to be miscalculated. Finally, since $P(r)$ assumes that particle emanate from a point source, it is not valid for highly focused streams and for very low standoffs where the size of the nozzle becomes appreciably large in proportion to the size of the scar radius at the surface.

It will be shown in Chapter 3 that the form of $P(r)$ can vary with particular blasting apparatus. For example, for the microblaster utilized in Chapter 3,P(r) takes a Weibull distribution, rather than the Gamma distribution proposed by Shipway and Hutchings [15] (equation (1.2)). The scar radius method thus also has limitations in predicting the onset of critical particle interference for distributions other than that defined by equation (1.2), which do not present as a linear relationship between scar radius and logarithm of mass of erodent. For the Gamma distribution, when the jet reaches a critical particle mass flux, $r$ begins to increase nonlinearly with the logarithm of the mass of erodent. But, for example, if the distribution of particles follows a Weibull distribution, $r$ increases nonlinearly with the logarithm of mass of erodent even at low flux. It would thus be difficult to define the transition point where the flux 
effects become critical since: 1) At that point, the nonlinear increase would change from a first to a second order, which is difficult to see; and 2) Scar radius experiments are difficult to perform [15-17] and hence the limited accuracy of the experimental data fit would make it even more difficult to find this transition point.

Particle interactions with the nozzle wall occur inside the nozzle, and are one of the main causes of particle jet divergence. Stevenson and Hutchings examined the influence of nozzle length and particle velocity on the divergence of the particle jet in a gas-blast erosion rig [16]. Normally, longer nozzles yielded higher particle velocities and decreased the variation of velocities across the jet, for varying particle sizes leaving the nozzle. However, this was found not to be necessarily true if the inside of the nozzle wall was rough, leading to reduced particle speeds due to the frictional effect and the onset of particle interactions inside the nozzle. Nozzle wall roughness increases jet divergence [15], as was previously discussed (i.e., the focus coefficient $\beta$ decreases). To study the effect of nozzle length on $\beta$, scar radius experiments were performed by blasting sand particles on flat aluminum targets covered with a thin dye at normal incidence. The flow rate was kept to a minimum in order to eliminate particle interference effects. Nozzle lengths of $85-1000 \mathrm{~mm}$ with a constant diameter of $4.9 \mathrm{~mm}$ were used. It was found that jet divergence rose rapidly with increasing particle velocity, at low velocities. However, at high velocities (i.e., beyond $40 \mathrm{~m} / \mathrm{s}$ ), this effect was found to be almost insignificant. In addition, it was found for a constant particle velocity of $50 \mathrm{~m} / \mathrm{s}$, the variation of nozzle size yielded a fairly constant $\beta$ (i.e., no variation in jet divergence). Hence, for applications requiring a very high particle velocity, it would be redundant to use a longer nozzle as they seem to have little effect on jet divergence. However, the nozzle length should be long enough to ensure that the erodent flow reaches steady state when exiting the nozzle tip. The investigators did not examine the use of shorter nozzles (i.e., on the order of 20-40 mm) and smaller nozzle diameters (on the order of $0.2-2 \mathrm{~mm}$ ), which might yield different results due to increased or decreased particle interactions inside the nozzle wall.

\subsubsection{Development of Profile Shape and its Application to Modeling Velocity Distribution in Micro-abrasive Jets}

Shipway further examined the influence of jet divergence on the particle spatial distribution and the erosion rate, as well as the development of surface profile shape in a gas- 
blast erosion rig at low flux conditions [17]. As before, it was assumed that plume divergence is caused by the internal nozzle wall roughness. He developed a computational model based on previous work $[15,16]$ that defines the angular distribution of particle trajectories. The model computes the effect of plume divergence on erosion rate and obtains the spatial distribution of wear. Erosion was performed on flat mild steel (ductile material) and glass (brittle material) samples using 125-150 $\mu \mathrm{m}$ silicon carbide particles. The eroded profile was analyzed using a stylus profilometer. The spatial distribution was assumed to follow equation (1.3). It was also assumed that particles arrive to the surface following straight trajectories, that there was axial symmetry in the jet, and that the velocity distribution across the jet was uniform. However, this is not always true as other investigators (Ref. [3] for example) have shown that the velocity distribution across the jet can be somewhat linear or parabolic, having its peak at the center and gradually decreasing with $r$. The erosion depth was calculated by multiplying $Q_{c r i t}$ (equation (1.4)) by the erosion rate at the true impact angle, which is different for ductile and brittle materials. The erosion rate-incident angle dependency was determined experimentally as the product of the erosion rate at normal incidence and a certain function of the impact angle. The simulated profile shapes did not match the experimental profiles very well for the ductile material and for high and low impact angles. Due to the exponential nature of equation (1.3), the computed profiles were very pointy at the center, whereas the experimental profiles had a more parabolic shape.

Other researchers have studied the development of the shape of an eroded profile in Abrasive Jet Micromachining (AJM). Balasubramanian et al. [18] developed a semi-empirical model for the erosion rate at the surface from fitting a simple particle mass distribution and particle number density profile. However, the model neglects the effect of particle interference which can affect the profile shape. In addition, the model neglects the change in the normal component of erosive energy as the surface profile progressively develops, and as a result works for only shallow profile depths. Achtsnick et al. [19] developed a simulation erosion model by assuming a classical indentation fracture mechanism for the interaction between particles and the surface. The model suffers from the same limitations as that of Ref. [18], and thus its application is limited to low flux conditions.

Slikkerveer and in't Veld [20] and Boonkkamp and Jansen [21] developed the most detailed analytical models to date for the development of the surface profile shape. The model in 
Ref. [21] relates the surface derivative at a point on the developing profile and the erosion rate via the normal velocity component. The model is very versatile as different erosion rates, particle mass fluxes, and velocity functions can be used. Unfortunately, the model does not consider multiple particle collisions and particle interference as the surface develops. Although the model in Ref. [20] further considered the initial particle rebound effect and the erosive effect of a second strike, the improvements were minimal. Overall, these models do not mimic experimental results where the profiles have a parabolic shape, especially near the center (as in Ref. [17]).

Among other researchers, Slikkerveer et al. [22] showed that the erosion rate depends on the magnitude of the particle impact velocity. Ballout et al. [23] along with Slikkerveer and in't Veld [20] and Boonkkamp and Jansen [21] also showed that for oblique incidence, the erosion rate is roughly proportional to the normal component of the particle impact velocity at the surface raised to a velocity exponent (which is a function of particle velocity, material and geometry, and the nature of the substrate). Furthermore, the depth at a point from the profile center was related to the erosion rate and the particle mass flux [20]. Using this relationship, it might be possible to obtain the velocity distribution from shallow profile depths. This will be investigated in Chapter 4. 


\subsection{Objectives, Motivation and Organization}

The objectives of this thesis are as follows:

1) To improve upon the limitations of the present simplified analytical models of particle interference for non-divergent jets of Refs. $[8,9]$ in order to predict the effective erosion rate at the surface and the critical flux below which particle interference would be negligible (Chapter 2).

2) To accurately characterize the divergent micro-abrasive particle jet by experimentally obtaining the particle spatial and velocity distribution and to improve the experimental techniques for obtaining these distributions (Chapters 3 and 4).

3) To use the results of 1) and 2) to formulate a more realistic analytical model for divergent jets that would predict the effective erosion rate at the surface and the critical flux below which particle interference would be negligible that could prove more useful in routine erosion testing (Chapter 5). 


\section{Chapter 2 Analytical Models of the Interference Effects in a Non- divergent Jet at Low Flux Conditions}

on pa

densit

will $b$

give $t$

\subsection{Motivation}

at low

$[10,12$

In solid particle erosion testing, the dependency of the erosion rate on incident particle flux can be attributed to the effects of interference between impacting particles, and those rebounding from the surface, as previously mentioned (e.g., [4,8-10,12]). These often neglected effects can significantly limit the erosive potential at the surface [3] and thus also have important implications for abrasive jet micromachining applications. As described in Chapter 1, computer simulations devised by Ciampini et al. $[10,12,13]$ allow the prediction of interference effects for multiple spherical particle collisions by tracking the movement and collision of individual particles in three-dimensions (3D), so that interference effects can be determined as a function of jet angle of attack, incident particle size and flux, nozzle size and divergence, and collision coefficients of restitution. While these computer simulations are generally applicable and thus very powerful, they are not readily available. However, analytical expressions can be used by anyone to obtain a quick, fairly accurate, estimate of the critical flux below which particle interference is deemed negligible. In addition, an analytical expression that could give the particle flux necessary to avoid significant particle interference effects would be more useful for routine erosion testing, where minimal computational complexity, time and cost is desired. Given their limitations, brought upon by the introduction of various assumptions, the analytical models have a useful range which is defined in this work. Beyond this range, the computer simulations of $[10,12,13]$ should be used to predict particle interference effects in erosion testing.

The analytical models of Andrews and Horsfield [8] and Anand et al. [9] discussed in Chapter 1 allow a basic understanding of particle interference effects. Andrews and Horsfield derived the probability of two particles colliding in a non-divergent particle jet, quantified the mean free path for a spherical particle, and found the variation of the relative energy of the scattered particles with the energy of the incident particles, as a function of the scatter angle [8]. Anand et al. derived the probability of collision between a single incident and a rebounding sphere at steady state in a non-divergent jet, and found an exponential dependence of erosion rate 
on particle mass flux [9]. However, these models do not account for radial symmetry, stream density, and general particle-to-surface collision energy losses. In this chapter, these analyses will be modified to take into account these effects, and analytical expressions will be derived that give the incident flux necessary for a certain level of particle interference for a non-divergent jet at low flux conditions. The results will then be compared to the computer simulation of Refs. $[10,12]$. Note that much of the material from this chapter will soon be published in Ref. [24]. 


\subsection{Modification of Existing Analytical Models of Particle Interference}

The analytical models of Refs. [8,9] are single collision models that consider only the first collision of an incident particle with a rebounding one in a non-diverging cylindrical jet. The probabilities of collision are derived considering only a single arriving and a single departing particle, representing the average probabilities of collision between incident and rebounding jets. It will be seen that this approach works well for incident angles less than $90^{\circ}$, when the size of the collision zone (i.e., the volume over which collisions can occur) near the surface remains relatively constant. Both models assume low flux conditions, a non-diverging incident jet of spherical particles (i.e., $\lambda=0$ ), a constant incident particle velocity $v_{a}$ (Figure 2.1) across the jet, and that particles are randomly located across the jet cross section. Both models also neglect energy loss in inter-particle collisions; in Ref. [8], energy loss in particle-surface collisions is neglected, while in Ref. [9], energy loss in particle-surface collisions is considered, but only for the direction normal to the surface (Figure 2.1). The following sections introduce modifications to account for radial symmetry, particle spacing, (i.e., the effect of stream density $[10,12])$, and particle-to-surface collision energy losses. 


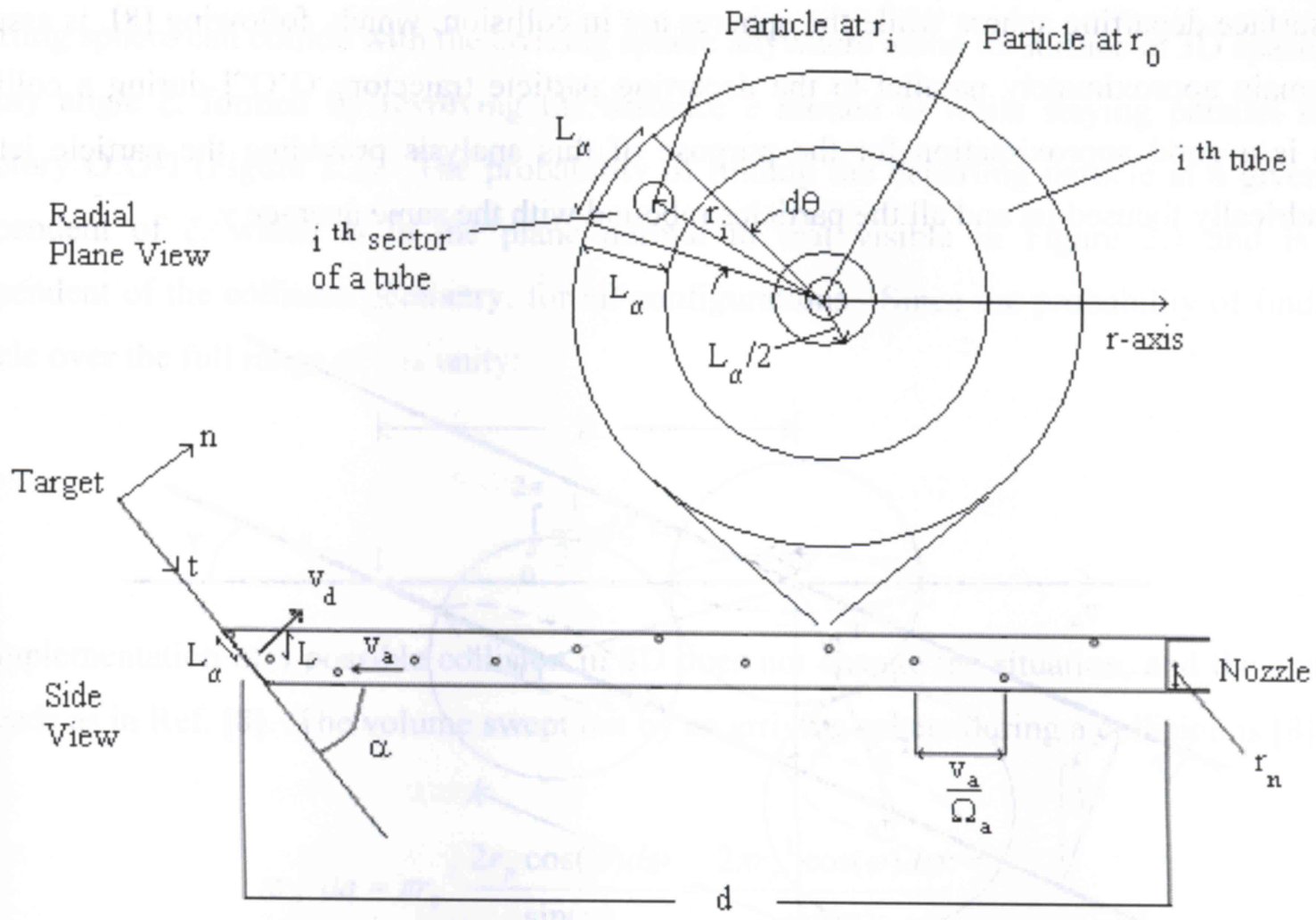

Figure 2.1: Side and cross sectional views of a particle jet impacting the target. The jet is divided into $i$ tubular regions, each containing particles located at a radial coordinate $r_{i}$, and a cylinder containing particles located at a radial coordinate $\boldsymbol{r}_{0}$.

\subsubsection{Model A: Modification of Method of Andrews and Horsfield [8]}

\subsubsection{Geometry of Collision between Two Spheres}

Figure 2.2, based on Figure A1 of Ref. [8], shows two particles, one from the jet arriving to the surface launched by a nozzle of radius $r_{n}$, and one from the jet departing from the surface, which is assumed flat. Following the method of Ref. [8], collision is assumed to occur when the distance between the two spheres is one diameter or less. Points O, O', and O" are the limiting positions of the sphere centroids for collision and the lines OI and O'O'I are the particle trajectories during collision, where $q$ is the distance traveled by the arriving sphere from I to O. $\gamma$ is the scatter angle, the angular range over which particles scatter following collisions with the surface, $r_{p}$ is the particle/sphere radius, and $v_{a}$ is the particle arrival velocity after being launched from the nozzle, which is assumed to be constant along $d$ (the standoff distance). $v_{d}$ is the departing particle velocity following a collision with the surface, and $c$ is the distance traveled by 
the surface departing sphere while the spheres are in collision, which, following [8], is assumed to remain approximately parallel to the departing particle trajectory O'O'I during a collision. This is a good approximation for the purpose of this analysis providing the particle jet is a cylindrically focused jet and all the particles rebound with the same average $\gamma$.

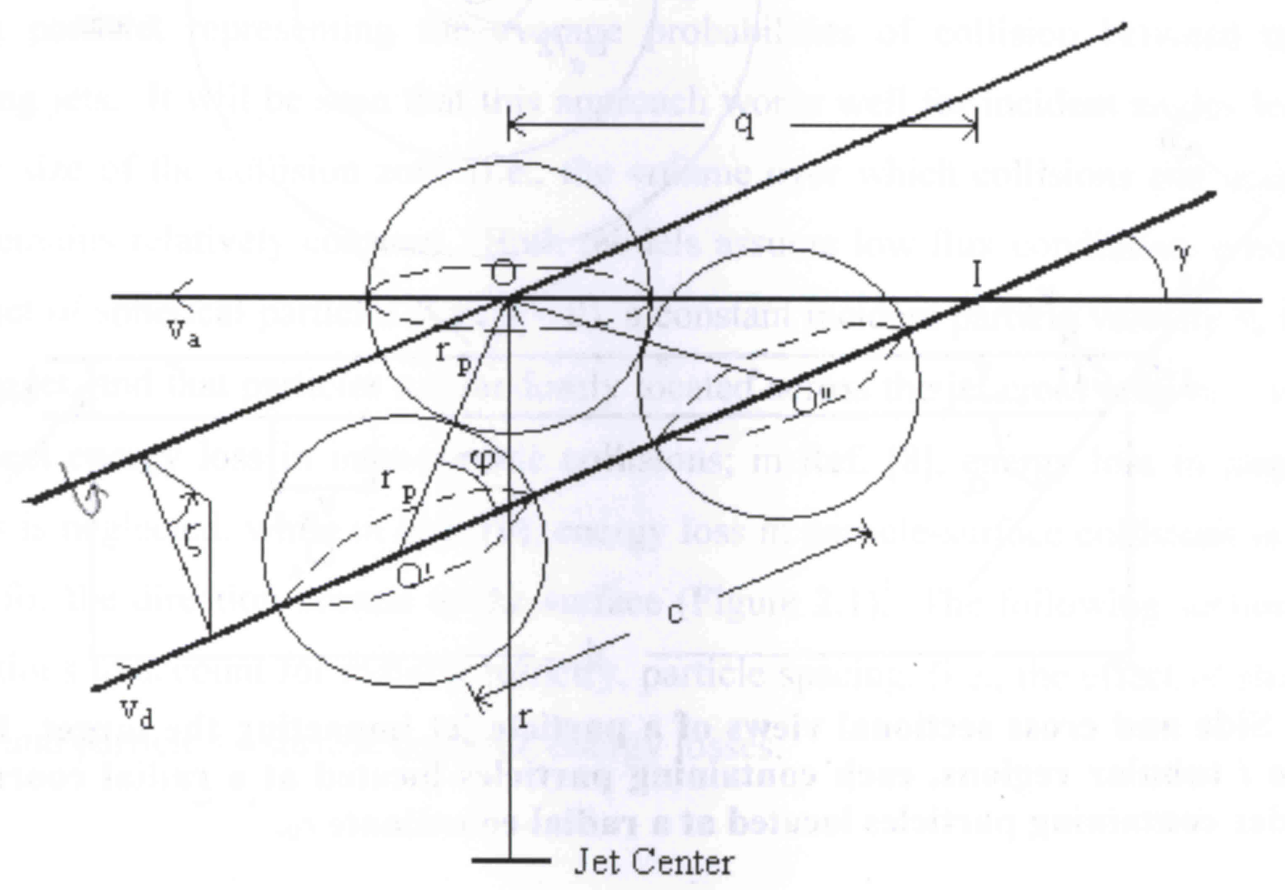

Figure 2.2: Collision between an arriving and departing particle (adapted from Ref. [8]).

Using trigonometric cosine and sine laws, respectively, (Figure 2.2) [8]:

$$
\begin{gathered}
c=2 r_{p} \sqrt{2-2 \cos (\pi-2 \varphi)}=2 r_{p} \sqrt{2+2 \cos (2 \varphi)} \\
q=\frac{2 r_{p} \sin (\pi-\varphi)}{\sin (\gamma)}=\frac{2 r_{p} \sin (\varphi)}{\sin (\gamma)}
\end{gathered}
$$

The limits of $\varphi$ are [8]:

$$
-\frac{\pi}{2} \leq \varphi \leq \frac{\pi}{2}
$$


While the analysis of Ref. [8] considered impacts in the plane of Figure 2.2, in reality, the departing sphere can collide with the arriving sphere anywhere along its surface in 3D space, i.e., for any angle $\xi$, formed by revolving the distance $c$ around $\mathrm{O}$ while staying parallel to the trajectory O'O'I (Figure 2.2). The probability of finding the departing particle at a given $\xi$ is independent of $\xi$, which is in the plane normal to that visible in Figure 2.1 and is thus independent of the collision geometry, for all configurations. Since the probability of finding a particle over the full range of $\xi$ is unity:

$$
\int_{0}^{2 \pi} \frac{1}{2 \pi} d \xi=1
$$

the implementation of a possible collision in 3D does not change the situation, and the analysis proceeds as in Ref. [8]. The volume swept out by an arriving sphere during a collision is [8]:

$$
\pi r_{p}{ }^{2} d q=\pi r_{p}{ }^{2} \frac{2 r_{p} \cos (\varphi) d \varphi}{\sin (\gamma)}=\frac{2 \pi r_{p}{ }^{3} \cos (\varphi) d \varphi}{\sin (\gamma)}
$$

and the volume swept by the departing sphere along $c$ during a collision [8], is:

$$
\pi r_{p}{ }^{2} c=2 \pi r_{p}^{3} \sqrt{2+2 \cos (2 \varphi)}
$$

These equations will be used in Section 2.2.1.3 to derive probabilities of collision between incident and departing spheres.

\subsubsection{Modification to Take into Account Particle Spacing and Radial Symmetry}

In Ref. [8], the locations of particles within the jet cross section of area $\pi r_{n}{ }^{2}$ are assumed to be random; i.e., particles are no more likely to appear at a given sector in Figure 2.1 than any other. However, the geometry and symmetry of the jet cross section imply that more particles can fit in the outer sectors in Figure 2.1, than at, for example, the center of the jet, an assumption built into the computer simulations of Refs. [10,12]. In the present analysis, this is accounted for by dividing the jet cross section into sectors along with one cylinder at the center (Figure 2.1), 
each with, on average, a particle located at the center. Rings formed by the outer sectors can thus accommodate more particles than the inner rings or the cylinder.

Let the particle jet be divided into $i$ concentric "tubes", each located at a radius $r_{i}$, having thickness $L_{\alpha}$, and one cylinder at $r_{0}=0$ (jet center) with a radius of $L_{\alpha} / 2$, (Figure 2.1). If $L$ is the mean distance between particles (for $\alpha=90^{\circ}$ ) in the radial direction (for a non-divergent jet) $[1,10]$, then:

$$
L_{\alpha}=\frac{L}{\sin (\alpha)}
$$

where

$$
L=2 r_{p} L^{*}=2 r_{p}\left(\frac{\pi}{6 \rho_{s}}\right)^{\frac{1}{3}}
$$

and $L^{*}$ is the dimensionless distance (in the radial plane) between incident particles. $\rho_{s}$ is the dimensionless stream density for a non-diverging jet (i.e., the ratio of the total volume of particles in the jet to the volume of the jet itself), defined as [12]:

$$
\rho_{s}=\frac{\frac{4}{3} \pi r_{p}^{3} \frac{\Omega_{a}}{v_{a}} d}{\pi r_{n}^{2} d}=\frac{4 r_{p}^{* 3}}{3 r_{n}{ }^{* 2}}
$$

where $r_{p}{ }^{*}$ and $r_{n}{ }^{*}$ are the dimensionless particle and nozzle radii, respectively; i.e., the number of particles leaving the nozzle in the time it takes a particle to travel a distance equal to $r_{p}$ or $r_{n}$ [12]:

$$
\begin{gathered}
r_{p}{ }^{*}=\frac{\Omega_{a} r_{p}}{v_{a}} \\
r_{n}{ }^{*}=\frac{\Omega_{a} r_{n}}{v_{a}}
\end{gathered}
$$


where $\Omega_{a}$ is the particle nozzle launch frequency (particles/s), and the expression $\frac{\Omega_{a}}{v_{a}} d$ in equation (2.9) is the total number of particles in the jet of length $d$. The amount of such tubes in the jet is approximated by $i_{\max }$ :

$$
i_{\max }=\frac{r_{n}-L_{\alpha} / 2}{L_{\alpha}}
$$

where the $i^{t h}$ tube $(i=1,2,3 \ldots)$ is located at $r_{i}$, for $r>0$ (Figure 2.1):

$$
r_{i}=i L_{\alpha}
$$

Given the above expressions, it is assumed that the particles are evenly distributed along the nozzle-to-surface standoff distance $d$, arriving one at a time, each separated by an average distance $v_{a} / \Omega_{a}$. Each particle can be in any sector of a tube at a particular $r_{i}$ in the nozzle plane since they all arrive randomly and are separated by the average distance $L_{\alpha}$ in each tube and across the nozzle plane (Figure 2.1). The maximum number of particles present at the center of the walls of each of the tubes (for $r>0$ ) in Figure 2.1 is $2 \pi r_{i} / L_{\alpha}$ so that each tube can be divided into $2 \pi r_{i} / L_{\alpha}$ sectors with, on average, a particle at the center. The volume swept by each arriving sector of a given tube spanned by $d \theta$ (where $d \theta=L_{\alpha} / r_{i}$ ) is:

$$
\left(\int_{r_{i}-L_{\alpha} / 2}^{r_{i}+L_{\alpha} / 2} 2 \pi r d r\right) \frac{d \theta}{2 \pi} \frac{v_{a}}{\Omega_{a}}=\left(2 \pi r_{i} L_{\alpha}\right)\left(\frac{L_{\alpha} / r_{i}}{2 \pi}\right) \frac{v_{a}}{\Omega_{a}}=L_{\alpha}{ }^{2} \frac{v_{a}}{\Omega_{a}}
$$

Following the arguments of Ref. [8], the probability of finding a particle in this volume is unity. Hence, the probability density per unit volume (the volume swept by each sector of a tube) of finding an arriving, or similarly, a departing particle at $r_{i}, P_{a, d e n}$ or $P_{d, d e n}$, is:

$$
\begin{aligned}
& P_{a, d e n}=\frac{\Omega_{a}}{v_{a} L_{\alpha}{ }^{2}} \\
& P_{d, d e n}=\frac{\Omega_{d}}{v_{d} L_{\alpha}{ }^{2}}
\end{aligned}
$$


where $\Omega_{d}$ (particles/s) is the frequency of particles departing the surface after colliding with it. Similarly, the volume swept out by a cylinder at $r_{0}$ (jet center) is:

$$
\left(\int_{0}^{L_{\alpha} / 2} 2 \pi r d r\right) \frac{v_{a}}{\Omega_{a}}=\frac{\pi}{4} L_{\alpha}{ }^{2} \frac{v_{a}}{\Omega_{a}}
$$

and the probability of finding a particle in this volume is unity, so that the probability density per unit volume of finding an arriving or departing particle at the jet center, $P_{a c, d e n}$ and $P_{d c}, d e n$ is:

$$
\begin{aligned}
& P_{a c, d e n}=\frac{4 \Omega_{a}}{\pi v_{a} L_{\alpha}{ }^{2}} \\
& P_{d c, d e n}=\frac{4 \Omega_{d}}{\pi v_{d} L_{\alpha}{ }^{2}}
\end{aligned}
$$

\subsubsection{Probability of Collision between Incident and Rebounding Particles at a Given Location in the Incident Jet}

Andrews and Horsfield's analysis [8] allowed calculation of the joint probability of arriving and departing spheres occupying the same space. Using a similar approach with equations (2.5) and (2.15) yields the probability of finding a colliding sphere in the arrival trajectory of each sector of a tube:

$$
\frac{2 \pi r_{p}{ }^{3} \Omega_{a} \cos (\varphi) d \varphi}{\sin (\gamma) v_{a} L_{\alpha}{ }^{2}}
$$

Similarly, using equations (2.6) and (2.16), the probability of finding a colliding sphere in the departing trajectory of each sector of a tube is:

$$
\frac{2 \pi r_{p}{ }^{3} \Omega_{d} \sqrt{2+2 \cos (2 \varphi)}}{v_{d} L_{\alpha}{ }^{2}}
$$


If it is assumed that the arrival times of spheres along the trajectories are random, as was the case in Ref. [8], the probabilities defined by equations (2.20) and (2.21) are independent, and the joint probability of arriving and departing spheres occupying the same space (i.e., the probability of them colliding, $\left.P_{\text {coll }}\right)$ is the product of the two:

$$
d P_{\text {coll }}=\frac{4 \pi^{2} r_{p}{ }^{6} \Omega_{a} \Omega_{d} \sqrt{2+2 \cos (2 \varphi)} \cos (\varphi) d \varphi}{\sin (\gamma) v_{a} v_{d} L_{\alpha}{ }^{4}}
$$

Integration of equation (2.22) over all possible configurations of $\varphi$, and multiplication of the result by equation (2.4), yields the probability of collision at a radius $r_{i}=i L_{\alpha}$, for $r>0$, as:

$$
P_{\text {coll }}{ }^{A}\left(r=r_{i}\right)=P_{\text {coll }}{ }^{A}=\frac{4 \pi^{2} \Omega_{a} \Omega_{d} r_{p}{ }^{6}}{\sin (\gamma) v_{a} v_{d} L_{\alpha}{ }^{4}} \int_{-\pi / 2}^{\pi / 2} \cos (\varphi) \sqrt{2+2 \cos (2 \varphi)} d \varphi=\frac{4 \pi^{3} \Omega_{a} \Omega_{d} r_{p}{ }^{6}}{\sin (\gamma) v_{a} v_{d} L_{\alpha}{ }^{4}}
$$

where the superscript A indicates the modified Andrews and Horsfield model. It is interesting to note that the probability of collision is independent of $r$ (i.e., it does not depend on which tube one considers). This is because the density of particles within a given tube (i.e., the ratio of particle volume to tube volume) is independent of $r$ [24]. With regards to collisions occurring at the center, following an identical procedure, use of equations (2.18) and (2.19) instead of equations (2.15) and (2.16), yields the probability of collision at $r_{0}$ :

$$
P_{\text {coll }}^{A}\left(r=r_{0}=0\right)=\frac{64 \Omega_{a} \Omega_{d} r_{p}{ }^{6}}{\sin (\gamma) v_{a} v_{d} L_{\alpha}{ }^{4}} \int_{-\pi / 2}^{\pi / 2} \cos (\varphi) \sqrt{2+2 \cos (2 \varphi)} d \varphi=\frac{64 \pi \Omega_{a} \Omega_{d} r_{p}{ }^{6}}{\sin (\gamma) v_{a} v_{d} L_{\alpha}{ }^{4}}
$$

\subsubsection{Probability of an Incident Particle Arriving to a Particular Surface Region without Colliding}

In their derivation, Andrews and Horsfield [8] defined $z$, the distance traveled by an arriving particle through a region of possible collisions, before finally colliding with a departing particle [8], after which it is assumed to no longer contribute to the surface flux. In reality $z$ varies with $r$, but using Figure 2.3, it can be approximated by $z=z_{\text {mean }}=r_{n}[\cot (\alpha)+\cot (\gamma)][8]$, 
where $z_{\text {mean }}$ (i.e., $z$ at $r=0$ ) is an average distance traveled by a particle through a region of possible collisions.

(a)

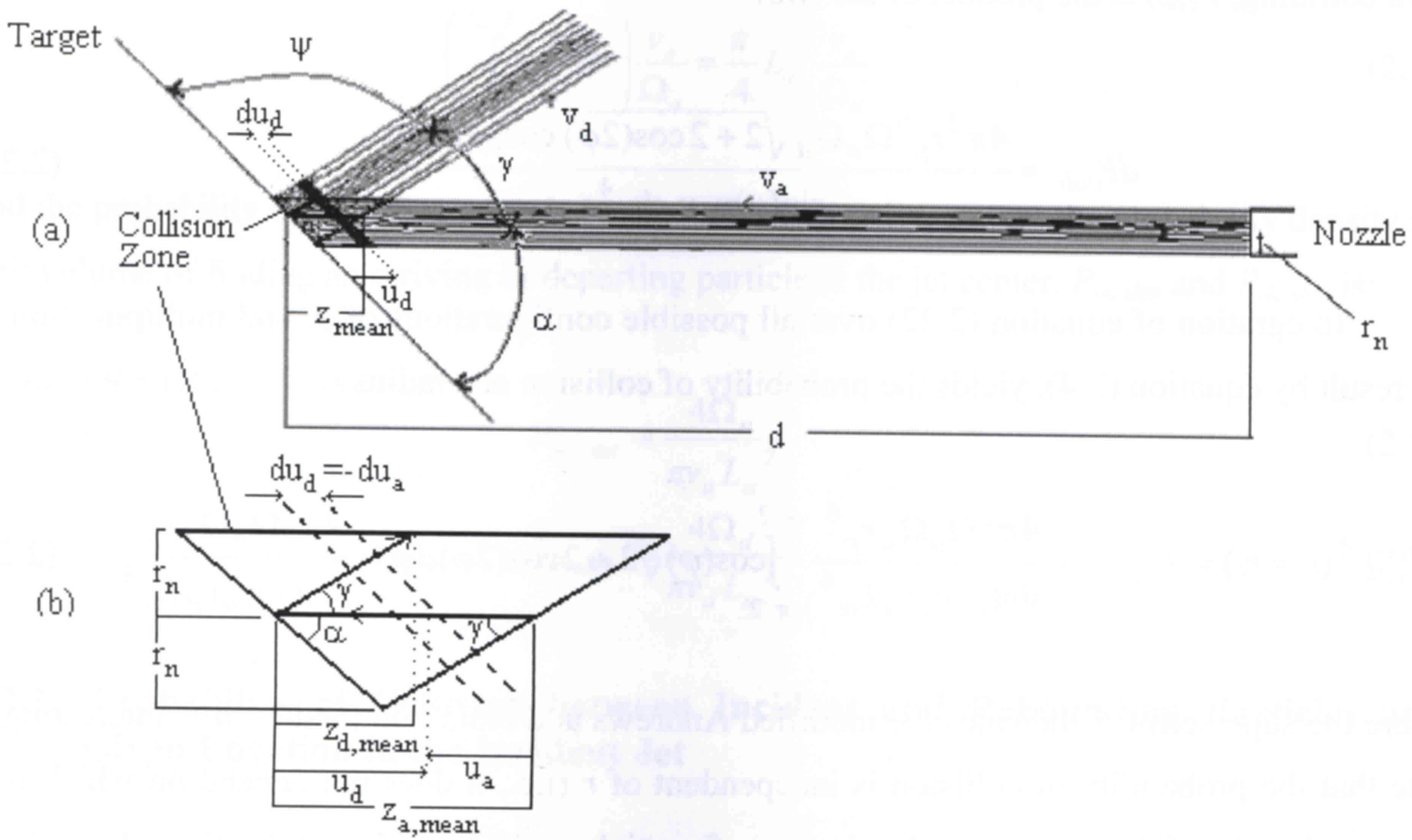

Figure 2.3: (a) Particle scattering, collision zone, and intersection of trajectories through a region of possible collisions; (b) Close up view of collision zone.

In the present work, for $z_{\text {mean }}$, a scatter angle of $\gamma=\pi-2 \alpha$ (i.e., $\psi=\alpha$, assuming no energy loss in a collision, where $\psi$ is the angle between the target surface and the departing trajectory (Figure 2.3 (a))) must be used to ensure that $z_{\text {mean }}$ remains strictly positive. For this case, using the fact that $\tan (\pi-2 \alpha)=-\tan (2 \alpha)$ and trigonometric identities for $\sin (2 \alpha)$ and $\cos (2 \alpha), z_{\text {mean }}$ is reduced to an approximation defined by:

$$
z_{\text {mean }}=r_{n}\left(\frac{1}{\tan \alpha}-\frac{1}{\tan (2 \alpha)}\right)=\frac{r_{n}}{\sin (2 \alpha)}
$$

which is the same as in Ref. [9] for $\alpha<90^{\circ}$ (see Section 4 of Ref. [9]). In Ref. [8], the number of intersections of a given average incident trajectory with the rebounding trajectories across a diametrical line in the rebounding jet was used to derive the probability of an incident particle surviving to a certain depth into the collision zone. In the present work, this is expanded to 
include the average number of intersections between an incident trajectory and rebounding trajectories in the entire rebounding jet cross section as follows (Figure 2.3 and 2.4):

$$
\frac{z_{\text {mean }} \tan \left(\frac{\gamma}{2}\right)}{2 r_{p}}\left(\frac{2 r_{n}}{2 r_{p}}\right)\left(\frac{\pi}{4}\right)=\frac{\pi z_{\text {mean }} \tan \left(\frac{\gamma}{2}\right) r_{n}}{8 r_{p}^{2}}
$$

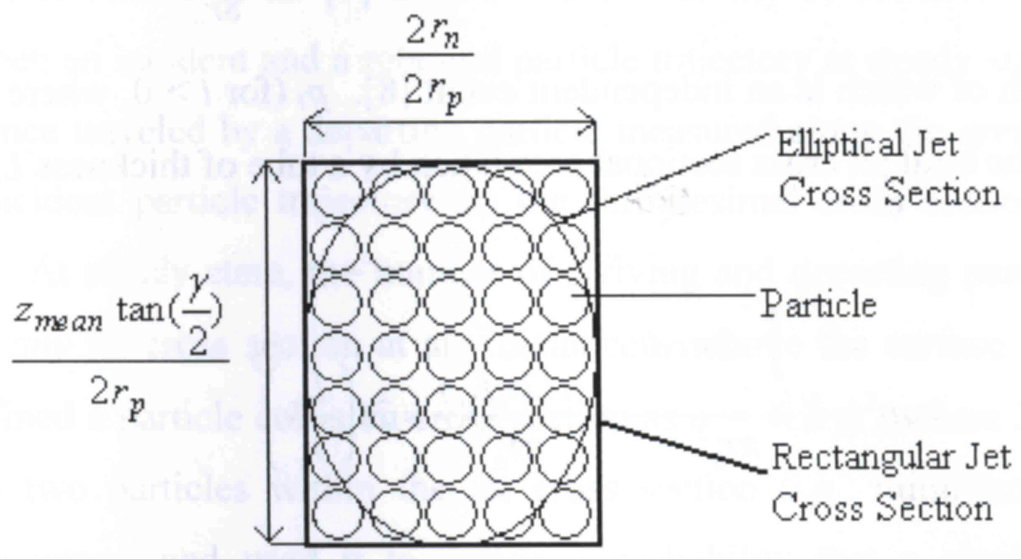

Figure 2.4: Estimation of the number of intersections between an incident trajectory and rebounding trajectories in the entire rebounding jet cross section.

The $2 r_{n} / 2 r_{p}$ factor converts the intersections of trajectories across the diametrical line in the rebounding jet given in Ref. [8] as $\frac{z_{\text {mean }} \tan \left(\frac{\gamma}{2}\right)}{2 r_{p}}$, to an estimate of the average number of intersections of trajectories in a $2 r_{n}$ by $z_{\text {mean }} \tan \left(\frac{\gamma}{2}\right)$ rectangular cross section. The $\pi / 4$ is an estimate of the ratio of the cross sectional areas of spheres fitting within an elliptical jet cross section to that of cross sectional areas of spheres fitting within a $2 r_{n}$ by $z_{\text {mean }} \tan \left(\frac{\gamma}{2}\right)$ rectangular jet cross section.

Following the reasoning of Ref. [8], and using equations (2.23) and (2.26), the probability of a particle surviving through the distance $z_{\text {mean }}$ and reaching the surface at a particular tube at radius $r_{i}=i L_{\alpha}, P_{\text {surv }}{ }^{A}\left(r_{i}\right)$, for $r>0$, becomes: 


$$
P_{\text {surv }}{ }^{A}\left(r_{i}\right)=\left[1-P_{\text {coll }}{ }^{A}\left(r_{i}\right)\right] \frac{\pi\left[z \text { mean } \cdot \tan \left(\frac{\gamma}{2}\right)\right] r_{n}}{8 r_{p}^{2}} p_{i}
$$

Equation (2.26) appears in the exponent of equation (2.27) because equation (2.27) signifies the probability of a particle surviving to the surface, through $\frac{\pi z_{\text {mean }} \tan \left(\frac{\gamma}{2}\right) r_{n}}{8 r_{p}{ }^{2}}$ possible intersections of trajectories, each of which is an independent event [8]. $p_{i}$ (for $i>0$, where $i$ is an integer), is the proportion of the total jet cross sectional area taken by a tube of thickness $L_{\alpha}$ located at $r_{i}$ :

$$
p_{i}=\frac{\int_{r_{i}-L_{\alpha} / 2}^{r_{i}+L_{\alpha} / 2} 2 \pi r d r}{\pi r_{n}^{2}}=\frac{\pi\left(2 r_{i} L_{\alpha}\right)}{\pi r_{n}^{2}}=2 i\left(\frac{L_{\alpha}}{r_{n}}\right)^{2}
$$

Similarly, for the $r=0$ case, the probability of a particle surviving through the distance $z_{\text {mean }}$ and reaching the surface at the center cylinder of radius $L_{\alpha} / 2$ is:

$$
P_{\text {surv }}{ }^{A}(r=0)=\left[1-P_{\text {coll }}{ }^{A}(r=0)\right] \underbrace{\left.\frac{\pi[z \text { mean }}{} \cdot \tan \left(\frac{\gamma}{2}\right)\right] r_{n}} p_{0}{ }^{2}
$$

where $p_{0}$ is the proportion of the total jet cross sectional area taken by a cylinder of radius $L_{\alpha} / 2$ located at $r_{0}$ :

$$
p_{0}=\frac{\int_{0}^{L_{\alpha} / 2} 2 \pi r d r}{\pi r_{n}^{2}}=\frac{1}{4}\left(\frac{L_{\alpha}}{r_{n}}\right)^{2}
$$

It should be noted that once $P_{\text {coll }}^{A}\left(r_{i}\right)$ or $P_{\text {coll }}^{A}(0)>1$, their respective probabilities of survival, $P_{\text {surv }}{ }^{A}\left(r_{i}\right)$ and $P_{\text {surv }}{ }^{A}(0)$, are 0 , as expected. It should also be noted that at $\alpha=90^{\circ}, z_{\text {mean }}=$ $\infty, P_{\text {surv }}=0$, and the present analysis cannot be used. Equations (2.27) and (2.29) can be used to estimate the percentage of particles surviving through a zone of possible collisions to a particular 
surface region defined by the intersection of the tube at $r_{i}$ or cylinder at $r_{0}$, with the surface plane.

\subsubsection{Model B: Modification of Method of Anand et al. [9] to Take into Account Particle Spacing and Radial Symmetry: Probability of an Incident Particle Arriving to a Particular Surface Region without Colliding}

The model of Anand et al. [9] derives the probability of collision by considering the intersection between an incident and a rebound particle trajectory at steady state. As in Ref. [9], let $u_{d}$ be the distance traveled by a departing particle measured along the arrival trajectory until it intersects an incident particle trajectory on the infinitesimal cross section of thickness $d u_{d}$ (Figure 2.3 (b)). At steady state, the number of arriving and departing particles per unit time must be equal at any jet cross section at any distance $u_{d}$ above the surface (i.e., $\Omega_{a}=\Omega_{d}$ ) [9]. Reference [9] defined a particle collision cross section as $\sigma=\pi\left(2 r_{p}\right)^{2}$, where $2 r_{p}$ is the minimum distance between two particles within the jet cross section (i.e., minimum $L_{\alpha}$ ) spanning all possible collision zones, and used it to define a probability that a single arriving particle undergoes a collision with any single departing particle at the cross section $d u_{d}, p_{\text {coll }}(r)$. The change in this probability over $d u_{d}, d p_{\text {coll }}(r)$, was then derived in terms of $d N$, the change in the number of departing particles per unit cross sectional area of the entire jet $\left(\pi r_{n}{ }^{2}\right)$, spanning the distance $d u_{d}$, as [9]:

$$
d p_{\text {coll }}(r)=\left[1-p_{\text {coll }}(r)\right](\sigma) d N=\left[1-p_{\text {coll }}(r)\right]\left(4 \pi r_{p}^{2}\right) d N
$$

where $\sigma d N$ is the total collision cross section per unit area (the probability that the particles will collide on $\left.d u_{d}\right)$, and $\left[1-p_{\text {coll }}(r)\right]$ is the probability that both the arriving and departing particle have not yet collided, and hence have reached the cross section $d u_{d}$ at $u_{d}$ [9]. In the present analysis, $d N$ is corrected to account for:

1) The amount of particles per unit cross sectional area for both the departing and arriving particle jet at the cross section of length $d u_{d}$ since both the arriving and departing particle fluxes contribute to the probability of collision between an arriving and a departing particle. 
2) Particle spacing and jet symmetry by using the same jet partitioning scheme used in Section 2.2.1.2.

In this context, let $u_{a}$ be the distance traveled by the arriving particle measured along the arrival trajectory until it intersects a departing particle trajectory on the cross section defined by the length $d u_{a}$, which has the same magnitude as $d u_{d}$ (Figure 2.3 (b)). Also, let $d N_{d}$ and $d N_{a}$ be the change in the number of departing and arriving particles per unit cross sectional area of an $L_{\alpha}$ thick tube at a radius $r_{i}$, (Figure 2.1), spanning the distance $\mathrm{d} u_{d}$ and $\mathrm{d} u_{a}$, respectively:

$$
\begin{aligned}
& d N_{d}=\Omega_{a} d u_{d}\left(v_{d} \int_{r_{i}-L_{\alpha} / 2}^{r_{i}+L_{\alpha} / 2} 2 \pi r d r\right)^{-1} \\
& d N_{a}=\Omega_{a} d u_{a}\left(v_{a} \int_{r_{i}-L_{\alpha} / 2}^{r_{i}+L_{\alpha} / 2} 2 \pi r d r\right)^{-1}
\end{aligned}
$$

Using the geometry of Figure 2.3 (b), for the departing particle jet, $z_{d, \text { mean }}=r_{n} \cot (\gamma)=r_{n} \cot (\pi$ $2 \alpha$ ), which is the average rebound length for the surface-departing particle jet over which collisions can occur, and for the arriving particle jet, $z_{a \text {,mean }}$ is defined in the same manner as in Section 2.2.1.4 by equation (2.25), all for $\alpha<90^{\circ}$. Following the reasoning of Ref. [9], the total probability of collision and survival, $P_{\text {coll }}{ }^{B}\left(r_{i}\right)$ and $P_{\text {surv }}{ }^{B}\left(r_{i}\right)$, can be obtained by integrating over all the possible collision zones from both the arriving and departing particle trajectories. Using the fact that $d u_{a}=\left|d u_{d}\right|$ (from $u_{a}=z_{d, \text { mean }}-u_{d}$ and since the probability of collision cannot be negative), equations (2.31) and (2.32) are combined to yield:

$$
\int_{0}^{P_{\text {coll }}{ }^{B}\left(r_{i}\right)} \frac{d p_{\text {coll }}\left(r_{i}\right)}{1-p_{\text {coll }}\left(r_{i}\right)}=\int_{0}^{z_{d, \text { mean }}} \frac{2 r_{p}{ }^{2} \Omega_{a} d u_{d}}{r_{i} L_{\alpha} v_{d}}+\int_{0}^{z_{a, \text { mean }}} \frac{2 r_{p}{ }^{2} \Omega_{a} d u_{d}}{r_{i} L_{\alpha} v_{a}}
$$

Hence, for $r>0$ : 


$$
P_{\text {surv }}{ }^{B}\left(r_{i}\right)=\left[1-P_{\text {coll }}{ }^{B}\left(r_{i}\right)\right]=e^{\frac{-\Omega_{a}}{2 i L_{\alpha}{ }^{* 2}} \cdot\left(\frac{z_{d, \text { mean }}}{v_{d}}+\frac{z_{a, \text { mean }}}{v_{a}}\right)}
$$

where $P_{\text {coll }}{ }^{B}\left(r_{i}\right)$ and $P_{\text {surv }}{ }^{B}\left(r_{i}\right)$ are defined in the same manner as in Section 2.2.1.4, the superscript B distinguishes this model from that in equation (2.29), and $r_{i}=i L_{\alpha}$ is used to identify a particular jet tube. It should be noted that to assess the probability of interference for the normal incidence case $\left(\alpha=90^{\circ}\right)$, Ref. [9] suggests using an arbitrary parameter of the order of $2 r_{n}$ to describe the distance over which a rebounding particle travels before colliding with an incident one. It is not clear, however, how this could be estimated, and thus only oblique incidence was considered herein.

In contrast to the model of Section 2.2.1, this approach yields a probability of collision which is dependent on $r$ since it is derived considering collision between an incident particle and a rebounding particle contained in a $L_{\alpha}$ thick tube at a radius $r_{i}$. For the jet center, $d N_{d c}$ and $d N_{a c}$ is the change in the number of departing and arriving particles per unit cross sectional area of the central cylinder at $r=0$ (Figure 2.1), spanning the distance $\mathrm{d} u_{d}$ and $\mathrm{d} u_{a}$, respectively:

$$
\begin{aligned}
& d N_{d c}=\Omega_{a} d u_{d}\left(v_{d} \int_{0}^{L_{\alpha} / 2} 2 \pi r d r\right)^{-1} \\
& d N_{a c}=\Omega_{a} d u_{a}\left(v_{a} \int_{0}^{L_{\alpha} / 2} 2 \pi r d r\right)^{-1}
\end{aligned}
$$

Following a similar procedure as was used to derive equation (2.34), using equation (2.35) in place of equation (2.32), gives the probability of survival at $r_{0}$ as:

$$
P_{\text {surv }}{ }^{B}(r=0)=\left[1-P_{\text {coll }}{ }^{B}(r=0)\right]=e^{\frac{-4 \Omega_{a}}{L_{\alpha}^{* 2} \cdot\left(\frac{z_{d, \text { mean }}}{v_{d}}+\frac{z_{a, \text { mean }}}{v_{a}}\right)}}
$$




\subsubsection{Models A and B: Modification to Include Energy Loss due to Particle-to- Surface Collisions}

In erosion testing, it is likely that a significant proportion of the particle's incident energy is lost in damaging the surface. This energy loss will affect the trajectories of particles rebounding from the surface, and thus the particle interference patterns. The modified analyses introduced in Sections 2.2.1 and 2.2.2 are now modified to include a coefficient of restitution approach developed by Brach [11]. The arrival velocity $v_{a}$ can be divided into velocity components normal (n) and tangential ( $\mathrm{t}$ ) to the surface (Figure 2.1), $v_{a n}=v_{a} \sin (\alpha)$ and $v_{a t}=$ $v_{a} \cos (\alpha)$, respectively. For the low friction case, when sliding after impact with the target occurs, using the fact that impulse equals change in momentum, neglecting particle rotational rebound velocity before and after impact with the target, and realizing that the arrival velocity varies only with $\alpha$ and no other out-of-plane angle, it can be shown that $[10,11] v_{d n}$ and $v_{d t}$, the departing normal and tangential particle velocity components, are, respectively:

$$
\begin{gathered}
v_{d n}=-e_{p s} v_{a n} \\
v_{d t}=f\left(v_{d n}-v_{a n}\right)+v_{a t}=-f v_{a n}\left(1+e_{p s}\right)+v_{a t}
\end{gathered}
$$

where $f$ and $e_{p s}$ are, respectively, the friction coefficient and coefficient of restitution, for particle-to-surface collisions. For the high friction case, when sticking after impact with the target occurs, $f$ in equation (2.38) is replaced by the critical impulse ratio, $\mu_{t}=2 v_{a t} /\left[7 v_{a n}\left(1+e_{p s}\right)\right]$ [11], which is obtained by setting expressions for the final tangential velocities in the sliding solution equal to those obtained in the rolling solution (obtained from conservation of angular momentum equation). The departing rotational velocity is ignored since the model only considers the first collision with the surface and neglects the rotational velocity in inter-particle collisions (since it is irrelevant due to the fact that the analytical models assume that both the arriving and the departing particle is removed from the analysis after a collision takes place $[8,9])$. The angle between the incident and departing jets (Figure 2.3) is now redefined as $\gamma=(\pi / 2$ $-\alpha)+\tan ^{-1}\left(v_{d t} / v_{d n}\right)$, which can then be substituted into equations (2.23), (2.24), (2.27), and (2.29), and the new magnitude of the surface departing velocity including restitution effects can now be obtained from equations (2.37) and (2.38), which can then be substituted into equations (2.23), 
(2.24), (2.27), (2.29), (2.34), and (2.36), to obtain the new probabilities of collision and survival for the modified analytical models introduced in Sections 2.2.1 and 2.2.2. 


\subsection{Percentage of Launched Particles Reaching the Entire Surface: Models A and B}

A simple assessment of particle interference effects in erosion testing is the percentage of particles reaching the entire target surface without undergoing prior collisions. This can be obtained by calculating the ratio of the number of particles surviving through a distance $z$ through the collision zone to the surface, $\Omega_{z}$ (particles/s), to the number of particles launched from the nozzle $\Omega_{a}$ (particles/s). Using the nomenclature of the present work, expressions giving $\Omega_{z} / \Omega_{a}$ from the original Andrews and Horsfield [8] and Anand et al. [9] models, for the case of $\alpha<90^{\circ}$ are, respectively:

$$
\begin{aligned}
& \frac{\Omega_{z}}{\Omega_{a}}=e^{\frac{-\pi\left(z_{\text {mean }}\right)\left(\Omega_{a}\right)^{2} r_{p}}{\cos ^{2}(\gamma / 2) v_{a} v_{d}}} \\
& \frac{\Omega_{z}}{\Omega_{a}}=e^{\frac{-4\left(z_{\text {mean }}\right)\left(\Omega_{a}\right) r_{p}{ }^{2}}{r_{n}{ }^{2} v_{d}}}
\end{aligned}
$$

where $z_{\text {mean }}$ is defined by equation (2.25).

In the context of the jet partitioning (i.e., the center cylinder and the $i$ tubular regions) scheme of Section 2.2.1.2, equations (2.27), (2.29), (2.34), and (2.36) represent the ratios of the number of particles in the region arriving to the surface without undergoing a collision, to the number of particles in that region. To calculate the proportion of total particles in the jet surviving to the surface, for both Models A (equations (2.27) and (2.29)) and B (equations (2.34) and (2.36)), the following equation can be used:

$$
\frac{\Omega_{z}}{\Omega_{a}}=p_{0} P_{\text {surv }}(r=0)+p_{i=1} P_{\text {surv }}\left(r_{i=1}\right)+\ldots+p_{i=i_{\max }} P_{\text {surv }}\left(r_{i=i_{\max }}\right)
$$

Since $i_{\max }$ is usually not an integer, the following cases are used in accordance with equation (2.41):

i) When $i_{\max } \leq 0$ there is only the cylindrical centre region and, 


$$
\begin{gathered}
\frac{\Omega_{z}}{\Omega_{a}}=p_{i_{\max }} P_{\text {surv }}\left(r_{i_{\max }}\right)=p_{0} P_{\text {surv }}(r=0) \\
p_{0}=\left(\frac{r_{n}}{r_{n}}\right)^{2}=1
\end{gathered}
$$

ii) When $0<i_{\max }<1$,

$$
\begin{gathered}
\frac{\Omega_{z}}{\Omega_{a}}=p_{0} P_{\text {surv }}(r=0)+p_{i_{\max }} P_{\text {surv }}\left(r_{i_{\max }}\right) \\
p_{i_{\max }}=1-p_{0}
\end{gathered}
$$

corresponding to the remainder of the total area of the nozzle, where the $i_{\max }^{\text {th }}$ ring has a thickness of less than $L_{\alpha}$ and thus equation (2.28) cannot be used. Equation (2.45) is also used in the exponent of equation (2.27) for $P_{\text {surv }}\left(r_{i_{\max }}\right)$ for Model A, since equation (2.27) contains $p_{i}$. For Model B, $r_{i \max }=1 \cdot L_{\alpha}$ is used in equation (2.34) for $P_{\text {surv }}\left(r_{i_{\max }}\right)$, since equation (2.34) contains $r_{i}$, where $\mathrm{i}_{\max }$ is rounded $u p$ to the nearest integer, to ensure a stable probability of survival.

iii) When $i_{\max }=1$, equation (2.44) is used with $p_{i_{\max }}=p_{1}$; i.e., equation (2.28), and $P_{\text {surv }}\left(r_{i_{\max }}\right)=P_{\text {surv }}\left(r_{1}\right)$ for both Models A and B.

iv) When $1<i_{\max }<2$,

$$
\begin{gathered}
\frac{\Omega_{z}}{\Omega_{a}}=p_{0} P_{\text {surv }}(r=0)+p_{1} P_{\text {surv }}\left(r_{1}\right)+p_{i_{\max }} P_{\text {surv }}\left(r_{i_{\max }}\right) \\
p_{i_{\max }}=1-p_{0}-p_{1}
\end{gathered}
$$


which is also used in the exponent of equation (2.27) for Model A, and $r_{i \max }=2 \cdot L_{\alpha}$ is used in equation (2.34) for Model B, for the same reason as those mentioned in case ii).

v) When $i_{\max } \geq 2$, the same procedure as outlined by i)-iv) is used.

By setting the left-hand side of equation (2.41) sufficiently high (e.g., 0.95, indicating that $95 \%$ of incident particles arrive to the surface without colliding), an estimate of the required maximum critical particle launch frequency (particles/s) for that level of particle interference, $\Omega_{a(c r i t)}$, can be extracted $[12,13]$. This can be easily related to the particle mass flow rate or mass flux:

$$
\dot{M}_{\text {crit }}=\Omega_{a(\text { crit })} m_{p}
$$

where $\dot{M}_{\text {crit }}$ is the critical mass flow rate $(\mathrm{kg} / \mathrm{s})$ and $m_{p}$ is the mass of one particle $(\mathrm{kg})$ :

$$
m_{p}=\frac{4}{3} \pi r_{p}^{3} \cdot \rho_{p}
$$

where $\rho_{p}$ is the particle mass density $\left(\mathrm{kg} / \mathrm{m}^{3}\right)$. Equation $(2.48)$ can be used as a guideline for performing erosion tests free of interference effects. As before, the level of interference that can be tolerated will depend on the application, but 0.95 , used in the work of Refs. [12,13], seems reasonable. 


\subsection{Results and Discussion}

\subsubsection{Comparison with Computer Simulation of Refs. [10,12]}

The results of the analytical models can be compared to a previously developed computer simulation which takes into account interference effects in particle jets [10,12], assuming a radially symmetric jet. The program ensures that every particle event is accounted for during execution, even if there are multiple surface or inter-particle collisions. For the data collected from the simulation corresponding to the parameters outlined in Table 2.1, a non-divergent jet, arriving to a rectangular $0.1 \mathrm{~m}$ by $0.1 \mathrm{~m}$ target, is assumed. The data are collected at steady state (approximately $10 \mathrm{~ms}$ of real time, corresponding to when the number of particles entering the system is equal to the number exiting). At maximum, 10,000 particles are in the simulation at a time. The coefficient of restitution for inter-particle collisions is assumed to be unity, since the analytical models do not account for energy loss in particle-to-particle interactions $[8,9]$.

Using equations (2.41)-(2.47) and the variables outlined in Table 2.1 (based on the typical values used in Refs. [10,12]), typical plots of $\Omega_{z} / \Omega_{a}$ versus $\rho_{s}$ and $\alpha\left(\alpha<90^{\circ}\right)$ are compared for the original models in Refs. [8,9], the modified models introduced in Sections 2.2.1 and 2.2.2, and the computer simulation (Figures 2.5-2.9).

Table 2.1: Variables used for comparison of analytical models with computer simulation.

\begin{tabular}{|c|c|c|c|c|c|}
\hline Variable & Figure 2.5 & Figure 2.6 & Figure 2.7 & Figure 2.8 & Figure 2.9 \\
\hline$v_{a}(\mathrm{~m} / \mathrm{s})$ & 120 & 120 & 120 & 120 & 120 \\
\hline$r_{p}(\mathrm{~m})$ & $\begin{array}{c}2 \times 10^{-5} \text { to } \\
1 \times 10^{-4}\end{array}$ & $\begin{array}{c}2 \times 10^{-4} \text { to } \\
1 \times 10^{-3}\end{array}$ & $2 \times 10^{-5}$ & $\begin{array}{c}2 \times 10^{-5} \text { to } \\
1 \times 10^{-4}\end{array}$ & $\begin{array}{c}2 \times 10^{-5} \text { to } \\
1 \times 10^{-4}\end{array}$ \\
\hline$r_{n}(\mathrm{~m})$ & $3.8 \times 10^{-4}$ & $3.8 \times 10^{-3}$ & $3.8 \times 10^{-4}$ & $3.8 \times 10^{-4}$ & $3.8 \times 10^{-4}$ \\
\hline$\Omega_{a}=\Omega_{d}$ & $6.64 \times 10^{5}$ & $6.64 \times 10^{5}$ & $6.64 \times 10^{5}$ & $6.64 \times 10^{5}$ & $6.64 \times 10^{5}$ \\
$($ particles $/ \mathrm{s})$ & & $60^{\circ}$ & $10^{\circ}$ to $90^{\circ}$ & $60^{\circ}$ & $60^{\circ}$ \\
\hline$\alpha($ degrees $)$ & $60^{\circ}$ & 1 & 1 & 0.5 & 1 \\
\hline$e_{p s}$ & 1 & 0 & 0 & 0 & 0.5 \\
\hline$f$ & 0 & 0.1 & 0.1 & 0.1 & 0.1 \\
\hline$d(\mathrm{~m})$ & 0.1 & $1.11-$ & 0.11 & $0.11-$ & 0.11 to \\
\hline$r_{p}{ }^{*}$ & 0.11 to & 5.53 & & 0.55 & 0.55 \\
\hline$r_{n}{ }^{*}$ & 0.55 & 21.03 & 2.10 & 2.10 & 2.10 \\
\hline$\rho_{s}$ & $4.08 \times 10^{-4}$ to & $4.08 \times 10^{-3}$ to & $4.08 \times 10^{-4}$ & $4.08 \times 10^{-4}$ to & $4.08 \times 10^{-4}$ to \\
& $5.1 \times 10^{-2}$ & 0.51 & & $5.1 \times 10^{-2}$ & $5.1 \times 10^{-2}$ \\
\hline
\end{tabular}




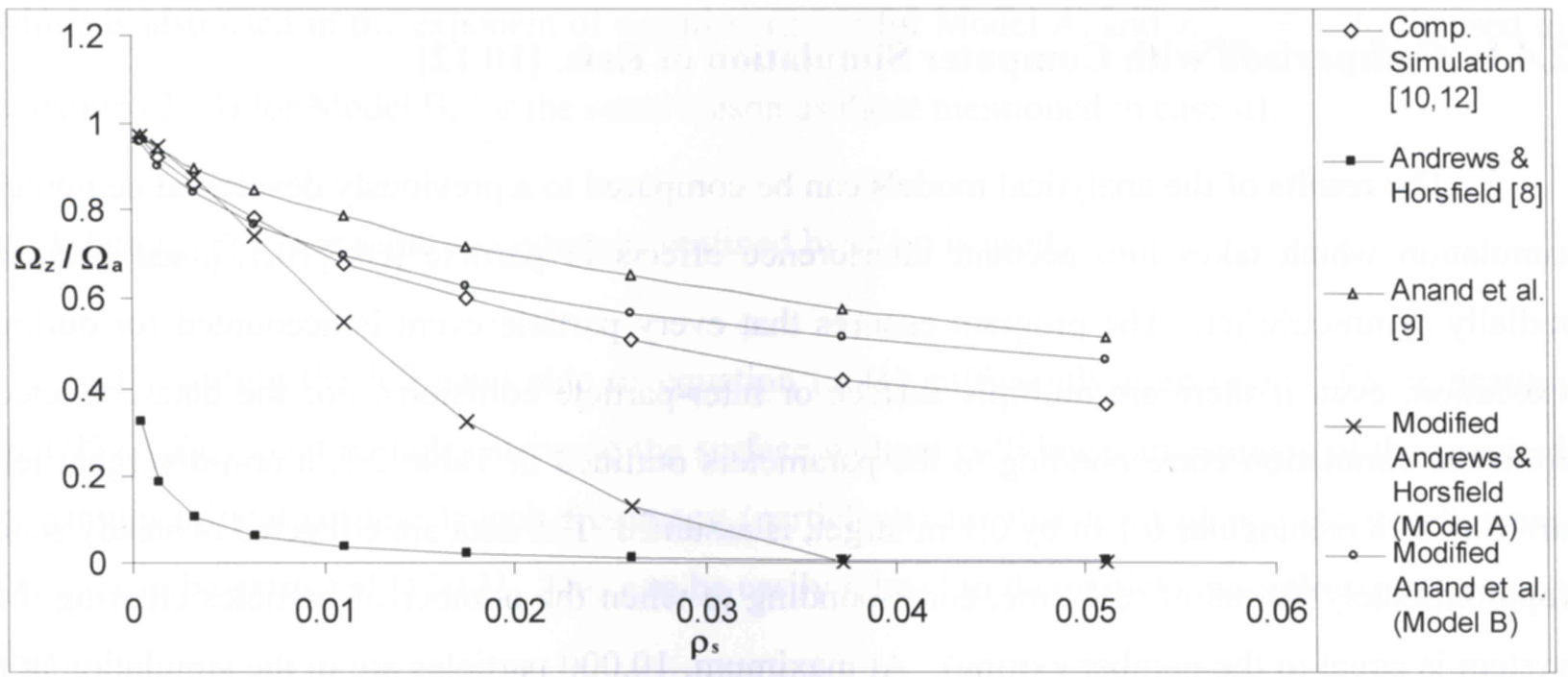

Figure 2.5: Effect of stream density, $\rho_{s}$ (lower range), on proportion of launched particles arriving to surface without having undergone a collision, $\Omega_{z} / \Omega_{a}$, for various models. Frictionless $(f=0)$ and elastic $\left(e_{p s}=1\right)$ particle-surface collisions assumed (Table 2.1).

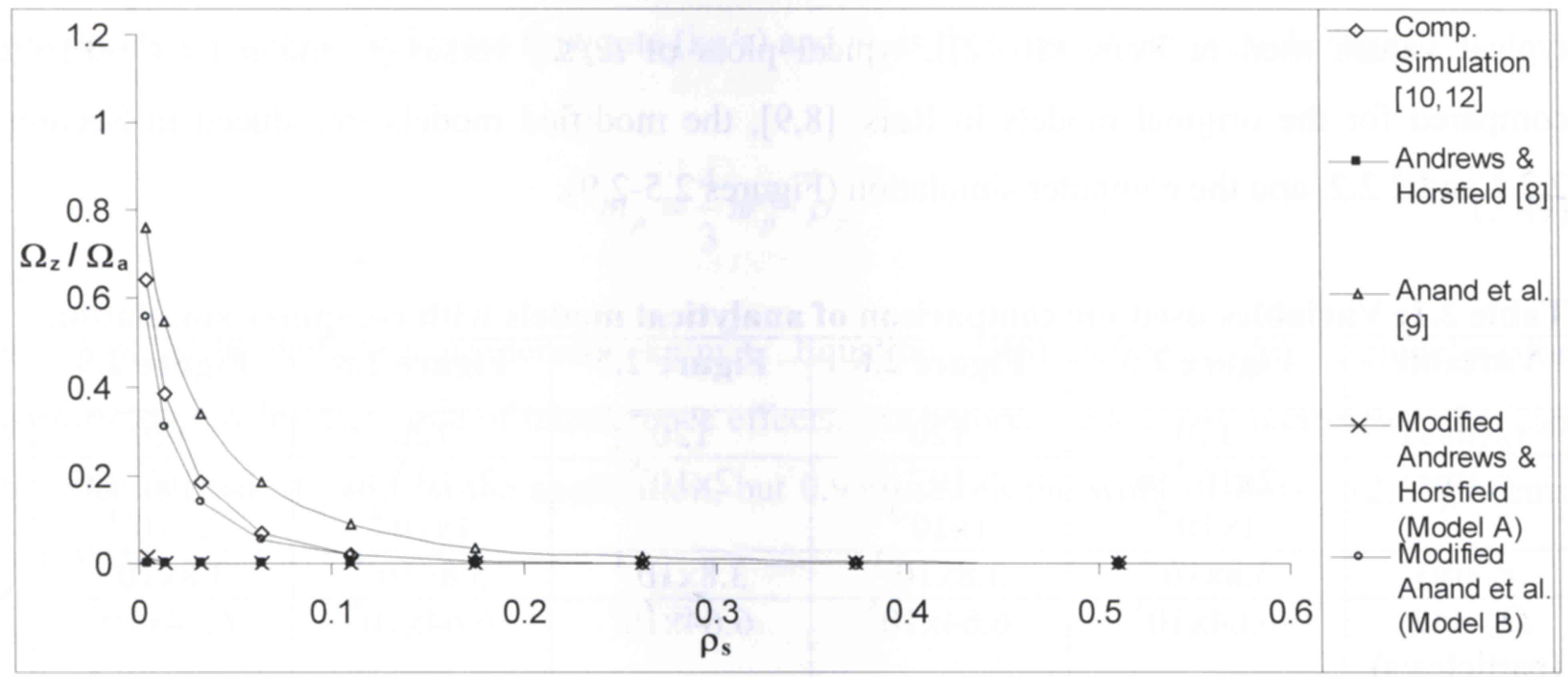

Figure 2.6: Effect of stream density, $\rho_{s}$ (higher range), on proportion of launched particles arriving to surface without having undergone a collision, $\boldsymbol{\Omega}_{z} / \boldsymbol{\Omega}_{a}$, for various models. Frictionless $(f=0)$ and elastic $\left(e_{p s}=1\right)$ particle-surface collisions assumed (Table 2.1). 


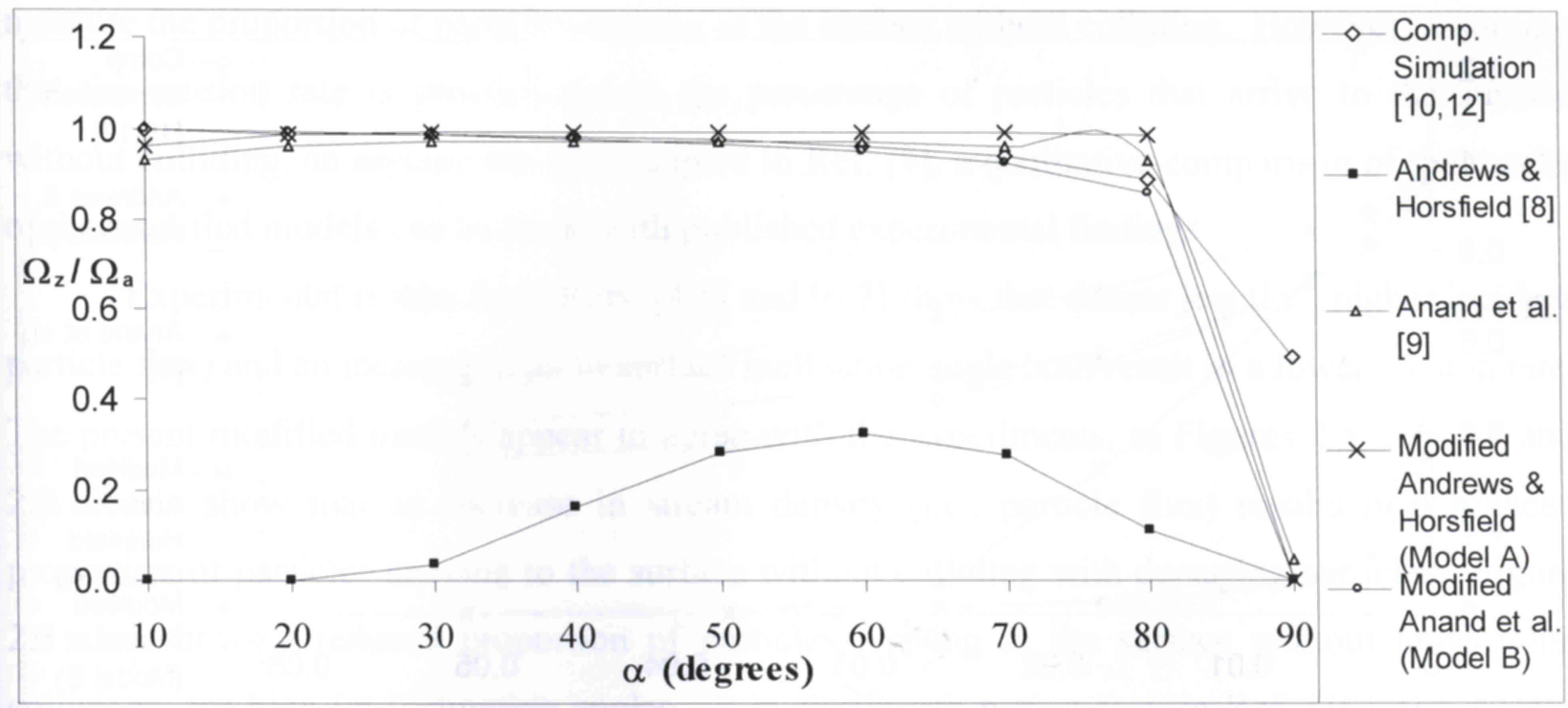

Figure 2.7: Effect of incident angle, $\alpha$, on proportion of launched particles arriving to surface without having undergone a collision, $\Omega_{z} / \Omega_{a}$, for various models. Frictionless $(f=0)$ and elastic $\left(e_{p s}=1\right)$ particle-surface collisions assumed (Table 2.1).

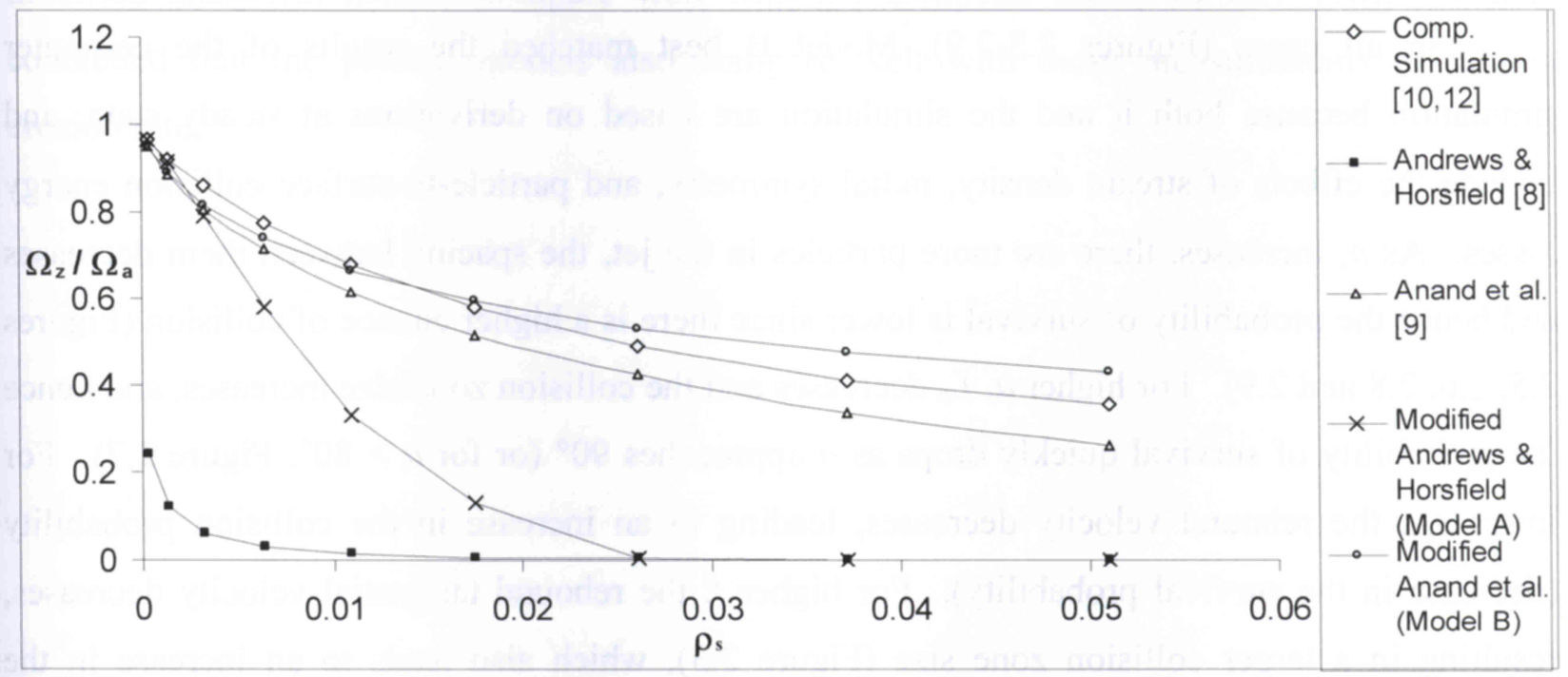

Figure 2.8: Effect of stream density, $\rho_{s}$ (lower range), on proportion of launched particles arriving to surface without having undergone a collision, $\boldsymbol{\Omega}_{z} / \boldsymbol{\Omega}_{a}$, for various models. Frictionless $(f=0)$ and inelastic $\left(e_{p s}=0.5\right)$ particle-surface collisions assumed (Table 2.1). 


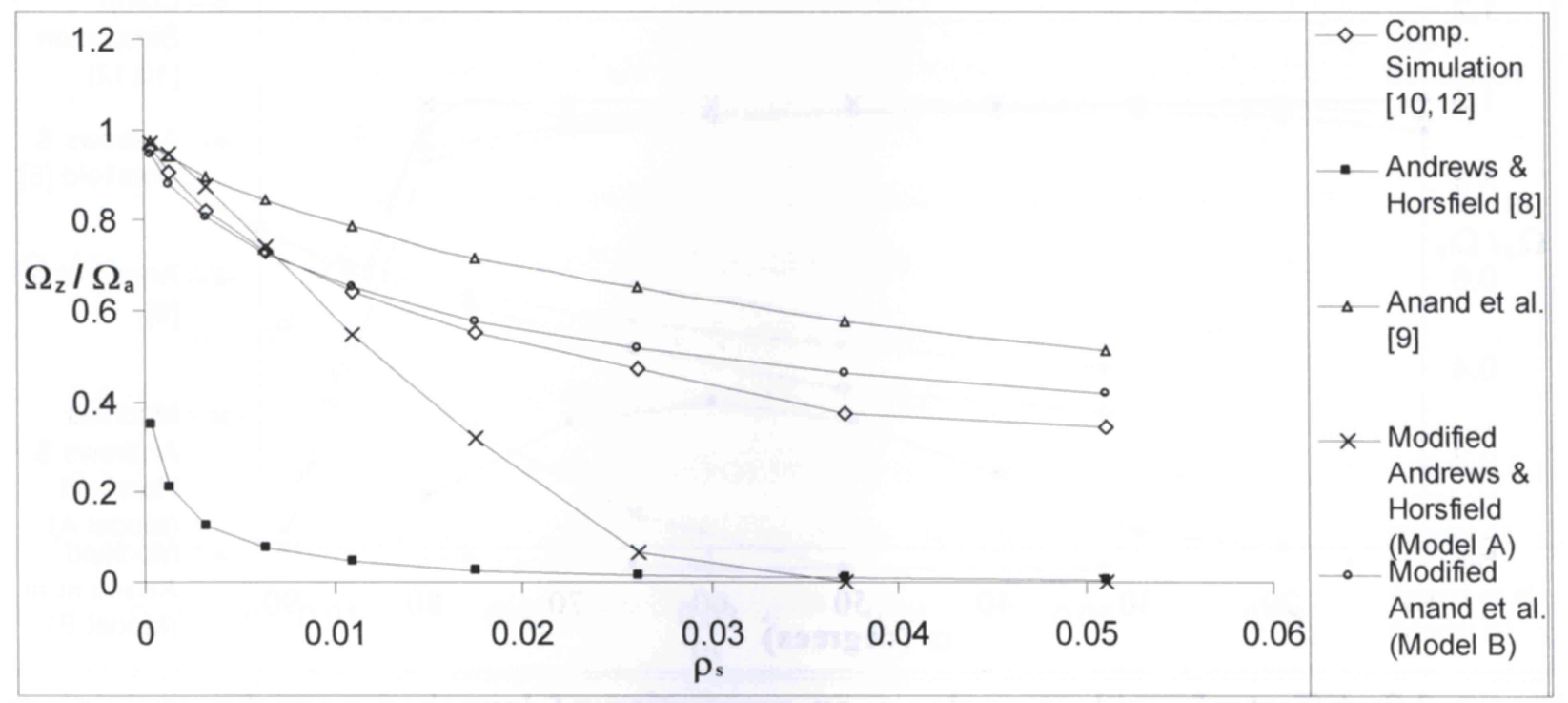

Figure 2.9: Effect of stream density, $\rho_{s}$ (lower range), on proportion of launched particles arriving to surface without having undergone a collision, $\boldsymbol{\Omega}_{z} / \boldsymbol{\Omega}_{a}$, for various models. Frictional $(f=0.5)$ and elastic $\left(e_{p s}=1\right)$ particle-surface collisions assumed (Table 2.1).

In all cases (Figures 2.5-2.9), Model $\mathrm{B}$ best matched the results of the computer simulation because both it and the simulation are based on derivations at steady state, and include the effects of stream density, radial symmetry, and particle-to-surface collision energy losses. As $\rho_{s}$ increases, there are more particles in the jet, the spacing between them decreases and hence the probability of survival is lower since there is a higher chance of collision (Figures 2.5, 2.6, 2.8 and 2.9). For higher $\alpha, L_{\alpha}$ decreases and the collision zone size increases, and hence the probability of survival quickly drops as $\alpha$ approaches $90^{\circ}$ (or for $\alpha>80^{\circ}$, Figure 2.7). For lower $e_{p s}$, the rebound velocity decreases, leading to an increase in the collision probability (decrease in the survival probability). For higher $f$, the rebound tangential velocity decreases, resulting in a larger collision zone size (Figure 2.3), which also leads to an increase in the collision probability (decrease in the survival probability). However, since the size of the collision zone is relatively small and remains relatively constant, variations in $e_{p s}$ and $f$ do not alter the survival probability by much (compare Figure 2.5 with Figure 2.8 and 2.9).

\subsubsection{Qualitative Comparison with Experimental Observations}

A direct comparison between the present model and experimental results is difficult to make, as, to the knowledge of the author, there appear to be no experimental studies which 
measure the proportion of particles arriving to the surface without colliding. However, assuming that the erosion rate is proportional to the percentage of particles that arrive to the surface without colliding, an assumption also adopted in Ref. [9], a qualitative comparison of the results of the modified models can be made with published experimental findings.

Experimental results from Refs. [4,5] and [6,7] show that denser jets (i.e., higher incident particle flux) and an increase in jet to surface inclination angle both result in a lower erosion rate. The present modified models appear to agree with the experiments, as Figures 2.5, 2.6, 2.8 and 2.9 clearly show that an increase in stream density (i.e., particle flux) results in a reduced proportion of particles arriving to the surface without colliding with departing particles. Figure 2.7 also shows a reduced proportion of particles arriving to the surface without undergoing collisions, for high jet inclination angles. It is also worth noting that, in Ref. [10], the surface erosive power availability predicted by the computer model was compared to the measured relative erosion rate (data from Ref. [4]) with reasonably good agreement. Since the present modified analytical models compare well with the computer model of Ref. [10], it can be concluded that the present models also compare well with those measurements of relative erosion rate. 


\section{Chapter 3 Measurement of the Particle Spatial Distribution in Micro- abrasive Jets}

\subsection{Motivation}

Blast cleaning, shot peening and abrasive jet micromachining (AJM) are industrial processes that involve jets of particles accelerated towards a target surface by a high-speed air jet. Blast cleaning uses an abrasive particle jet to remove a coating or contaminants from substrate surfaces. Shot peening is a process where spherical particles are bombarded on uncoated metal substrates in order to induce compressive residual stresses, which prevents the onset of surface fatigue cracks. AJM uses a small high-speed air jet to machine microstructures via the use of masks and by varying jet parameters such as velocity, angle of incidence, particle flux, and particle material and geometric properties [25]. These processes require high degrees of precision and repeatability.

In all such processes, in order to control and predict the particle interference effects, and hence the resulting shape of the eroded profile on the target surface, the particle jet must be accurately characterized by obtaining the particle spatial and velocity distribution across the jet $[15,17,25]$. This chapter describes a novel technique for obtaining the spatial distribution, by direct particle capture. The spatial distribution is needed as an input for an analytical model that can realistically predict particle interference and surface erosion rates for a diverse set of conditions (Chapter 5).

Portions of the material in this chapter are taken from Ref. [25], which will soon be published, and Ref. [26], which is already published. 


\subsection{Measurement of Particle Spatial Distribution across the Jet \\ 3.2.1 Experimental Setup and Procedure}

Angular $10 \mu \mathrm{m}$ and $25 \mu \mathrm{m}$ nominal diameter alumina $\left(\mathrm{Al}_{2} \mathrm{O}_{3}\right)$ particles and $50 \mu \mathrm{m}$ spherical silica glass $\left(\mathrm{SiO}_{2}\right)$ particles (Comco Inc., Burbank, $\mathrm{CA}$, USA) having mass densities of $3900 \mathrm{~kg} / \mathrm{m}^{3}$ and $2480 \mathrm{~kg} / \mathrm{m}^{3}$, respectively, were used in all experiments. The particles were blasted using a microblaster (Comco Inc., Burbank, CA, USA) having the following specifications (Figure 3.1):

- Model: MB 1005

- Weight: $9.5 \mathrm{~kg}$

- Size: $230 \mathrm{~mm}$ x $435 \mathrm{~mm}$ x $255 \mathrm{~mm}$ (height $\mathrm{x}$ width $\mathrm{x}$ depth)

- Operating pressure (high pressure): $270-1,340 \mathrm{kPa}$

- Inlet air pressure (high pressure): $550-1,380 \mathrm{kPa}$

- Media Capacity: $820 \mathrm{~cm}^{3}$

- Air volume: $235-950 \mathrm{~cm}^{3} / \mathrm{s}$

- Air supply quality: less than $200 \mathrm{ppm}$ of moisture and less than $10 \mathrm{ppm}$ of oil content

- Power requirements: $115 \mathrm{~V} / 60 \mathrm{~Hz}$ or $230 \mathrm{~V} / 50 \mathrm{~Hz}, 40 \mathrm{~W}$

Mass flux repeatability was improved by adding a mixing device (a rod with a blade at one end connected to a D.C. motor at the other) to the pressurized particle reservoir (Figure 3.1). In order to eradicate mass flow and pressure fluctuations at start-up, a blast shutter [25], consisting of an aluminum plate connected to two fast-acting opposing solenoids, was positioned between the nozzle and the target for 20 seconds to allow the jet to reach steady state. A digital controller then actuated one of the solenoids, lifting the plate out of the jet path for the preset exposure time, after which the action of the solenoids was reversed, moving the plate back into the path of the jet (Figure 3.2). 


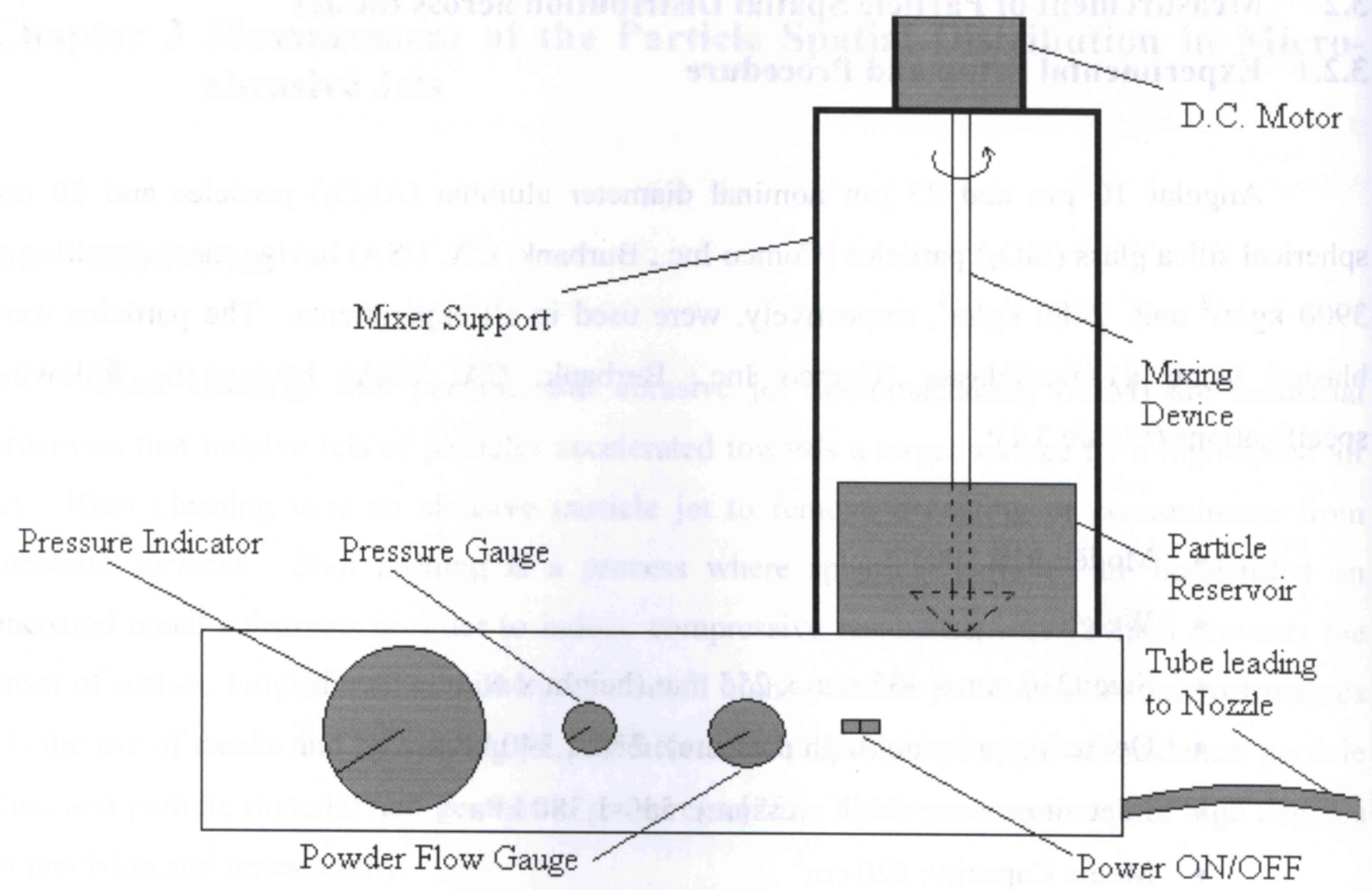

Figure 3.1: Schematic of microblaster.

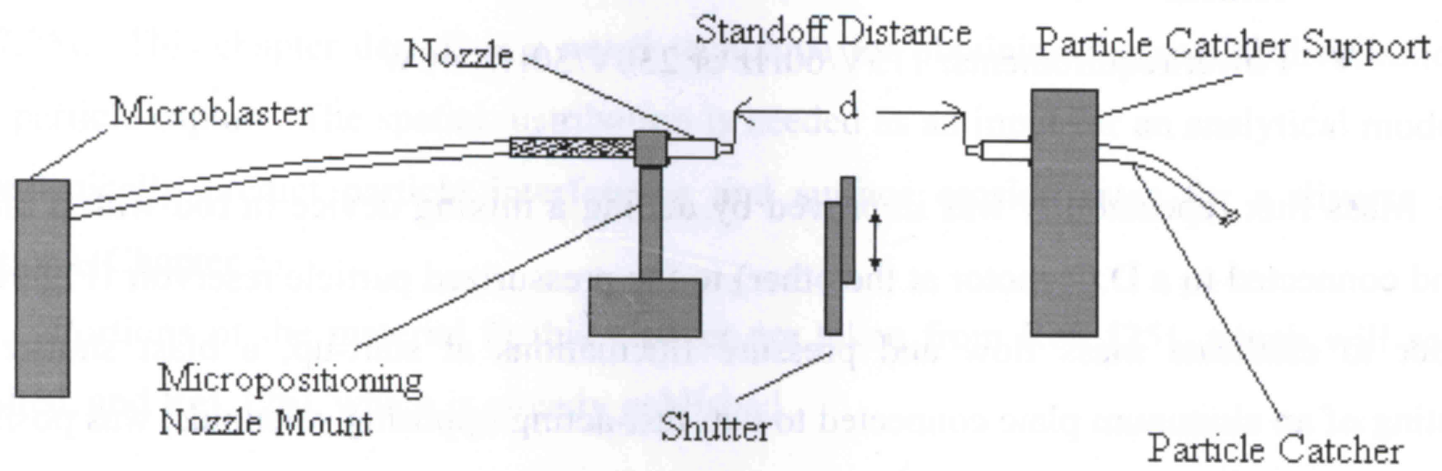

Figure 3.2: Schematic of experimental setup (side view).

The spatial distribution of particles across the jet, using three round $(0.46 \mathrm{~mm}$ and 1.5 $\mathrm{mm}$ high performance (H.P.) and $0.76 \mathrm{~mm}$ inner diameter) and two rectangular $(3.2 \times 0.2 \mathrm{~mm}$ and $3.8 \times 0.3 \mathrm{~mm}$ ) nozzles (Comco Inc., Burbank, CA, USA), was measured using a particle collection technique previously developed by the author [27]. All experiments were performed at normal incidence (i.e., $\alpha=90^{\circ}$, where $\alpha$ is the angle of incidence the nozzle makes with the surface) at 
mass flow rates between $0.23-4.75 \mathrm{~g} / \mathrm{min}$ with standard deviations between $1.2-25 \%$. For the large majority of cases the standard deviations fell in the range of 1.2-4.2\%. Two blasting pressures were chosen: 200 and $300 \mathrm{kPa}$. The nozzle-to-target distances, $d$ (standoff), were between 10 and $40 \mathrm{~mm}$, depending on the blasting conditions and the effect being measured (i.e., for smaller nozzles, $d$ was reduced to obtain measurable results, and for alumina particles, $d$ was increased to minimize target wear).

The particle mass flow rates for the various blasting conditions were measured by blasting particles for 1 minute at steady state into a container through an opening just big enough to fit the nozzle tip. This ensured that none of the particles escaped during the collection process. The container was weighed before and after-using a typical microbalance, accurate to $0.1 \mathrm{mg}$, to obtain the collected mass, and the measurements were repeated twice in order to obtain a fair estimate of the mass flow rate. The mass flow rate measurements along with their corresponding standard deviations are recorded in Appendix A, Tables A-1b-A-18b, and in Table 3.1 .

To measure the particle distribution, a burst of particles was launched using the microblaster and shutter setup, and a tungsten carbide tube connected to a nylon hose (i.e., the 'catcher'), with the tube axis parallel to the nozzle axis (Figures 3.2, 3.3), was used to collect particles [25,26,27]. The mass of particles collected over varying time intervals $(60,90,120$, 180 or $240 \mathrm{~s}$, depending on the blasting conditions) was measured as a function of the radius, $r$, by positioning the catcher at various positions in the jet, using a micropositioning nozzle mount (Edmund Optics Inc., Barrington, NJ, USA), accurate to $100 \mu \mathrm{m}$, and weighed using the same microbalance used in obtaining the mass flow rates. The exposure time was chosen as a compromise between minimizing catcher wear, whilst simultaneously collecting enough particle mass to ensure measurable results.

Wear of the collection cylinder face was only a concern when using alumina particles. For these cases, an empty collection cylinder was weighed before and after each experimental run and was found to experience negligible mass loss (i.e., on the order of $100 \mu \mathrm{g}$ ). Also, during the course of all of the experimental runs, the collection cylinder was replaced several times with a new one in order to minimize this effect.

In the case of $10 \mu \mathrm{m}$ angular alumina, the particles had a tendency to stick together and agglomerate into clumps during the blasting process, even before leaving the nozzle. The mixing 
device depicted in Figure 3.1 limits this effect on the microblaster side, but when the particles came into contact with the inner wall of the catching tube, some particle agglomeration was observed. When a small catching tube diameter was used, the entrance tended to get clogged rather quickly, preventing other particles from entering the collecting tube. As a result, a larger opening was needed to ensure that the correct amount of mass was collected. Thus, for experiments involving $10 \mu \mathrm{m}$ angular alumina, the catching tube had an inner diameter of $2 r_{c}=$ $1.2 \mathrm{~mm}$ and a minimal wall thickness of $780 \mu \mathrm{m}$. For all other experiments, the catching tube had an inner diameter of $0.46 \mathrm{~mm}$ and a minimal wall thickness of $100 \mu \mathrm{m}$ since the larger particles did not appear to stick together. The small wall thickness minimized interference from particles rebounding from the rim of the catching tube.

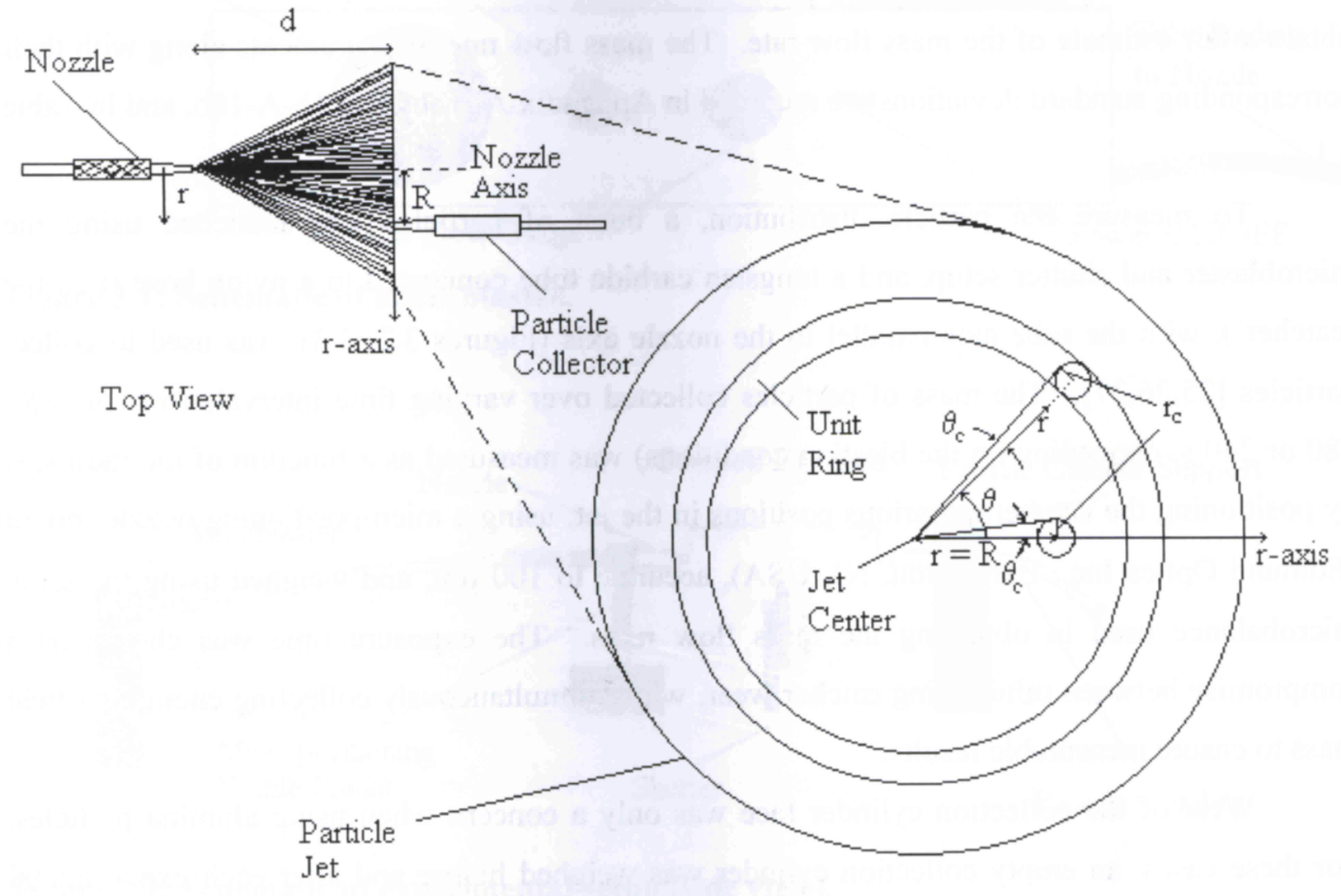

Jet Cross Sectional View

Figure 3.3: Particle collection apparatus for measurement of particle spatial distribution across jet (top view along with the jet cross sectional view). 
For ease of presentation of the results, non-dimensional coordinates (designated with an asterix superscript) were used, using the normalizing factor of the nozzle to surface standoff distance, $d$; i.e., the normalized radial coordinate $r^{*}=r / d$. For round nozzles, the mass collected in the particle collector at a specific non-dimensional position $r^{*}=R^{*}=R / d$ was designated as $M\left(R^{*}\right)$, where $R^{*}$ is the non-dimensional distance from the jet center to the center of the collection cylinder (Figure 3.3). $M\left(R^{*}\right)$ was then normalized by the mass collected at the jet center, $M(0)$, and plotted as a function of the non-dimensional radial coordinate, $r^{*}=r / d$ (Figures 3.4-3.13, Section 3.3.1) [25]. For rectangular nozzles, the mass collected in the particle collector at a specific non-dimensional position along the center line $x^{*}=X^{*}=X / d$ or $y^{*}=Y^{*}=Y / d$ (Figure 3.16) were designated as $M\left(X^{*}\right)$ or $M\left(Y^{*}\right)$, respectively, where $X^{*}$ and $Y^{*}$ are the non-dimensional distances from the jet center to the center of the collection cylinder measured along the $x$ - and $y$ axis. $M\left(X^{*}\right)$ and $M\left(Y^{*}\right)$ were then normalized by the mass collected at the jet center, $M(0)$, and plotted as functions of the non-dimensional coordinates $x^{*}=x / d$ and $y^{*}=y / d$, respectively (Figures 3.14-3.15, Section 3.3.2). All of the experiments were repeated twice (Tables A-1a-A$18 \mathrm{a}$ ), and the data were found to be very repeatable, with standard deviations for the two sets of data for each experiment, for all of the experiments, varying between $0-5.79 \%$ (Figures 3.4$3.15)$.

\subsubsection{Analysis}

The measured non-dimensional mass collected at a particular collection location (i.e., at $r$ $=R), M(R) / M(0)$, can be related to the joint probability of finding a particle between $r$ and $r+$ $d r, P(r) d r$, and the probability of finding a particle between $\theta$ and $\theta+d \theta, G(\theta) d \theta$, as follows [25]:

$$
\frac{M(r=R)}{M(r=0)}=\frac{\int_{-\theta_{c}}^{\theta_{c}} G(\theta) d \theta \cdot \int_{R-r_{c}}^{R+r_{c}} P(r) d r}{2 \int_{-\pi / 2}^{\pi / 2} G(\theta) d \theta \cdot \int_{0}^{r_{c}} P(r) d r}
$$


where $r_{c}$ is the inner radius of the cylindrical particle collector, and $\theta_{c}$ is the angle shown in Figure 3.3 for the limiting case $r=R\left(\theta_{c}\right.$ is a function of $\left.r\right)$. Since the jet is axis-symmetric, particles were no more likely to arrive at any given $\theta$, and thus:

$$
G(\theta)=\frac{1}{2 \pi}
$$

The range of $\theta$ (Figure 3.3) spanned at a given $r$, for $r>0$, with the particle collector centered at $r=R$, can be obtained by solving:

$$
(r \cos (\theta)-R)^{2}+(r \sin (\theta))^{2}=r_{c}^{2}
$$

for:

$$
\theta_{c}=\cos ^{-1}\left[\frac{r^{2}+R^{2}-r_{c}^{2}}{2 r R}\right]
$$

The range of $\theta$ spanned at $r=0$ is $\pi / 2$, giving $G(\theta) d \theta=1 / 2$ when integrated over the range of $\pi / 2$ to $\pi / 2$. Applying equations (3.2)-(3.4), along with $r^{*}=r / d, R^{*}=R / d$ and the fact that $P(r) d r=P\left(r^{*}\right) d r^{*}$, equation (3.1) becomes:

$$
\frac{M\left(R^{*}\right)}{M(0)}=\frac{\int_{R^{*}-r_{c}^{*}}^{R^{*}+r_{c}^{*}}\left[\frac{\cos ^{-1}\left(\frac{r^{* 2}+R^{* 2}-r_{c}^{* 2}}{2 r^{*} R^{*}}\right)}{\pi}\right] P\left(r^{*}\right) d r^{*}}{\int_{0}^{r_{c}^{*}} P\left(r^{*}\right) d r^{*}}
$$

where $r_{c}{ }^{*}=r_{c} / d$ is the non-dimensional collection cylinder radius.

The Romberg method was used to evaluate the integral of equation (3.5) with the numerical error controlled by a built-in tolerance variable in the Mathcad 11 software (Mathsoft 
Inc., Cambridge, MA, USA) set at 0.001. In this method, trapezoidal approximations are used over an even number of subintervals. Sequential estimates are compared by summing the areas of the trapezoids. The method terminates when the four most recent estimates differ by less than the value of the built-in variable TOL (0.001). Since the numerator of equation (3.5) represents the proportion of total particles launched that are collected in the particle catcher at a given $R^{*}$ location, and the denominator represents the proportion of total particles collected at $R^{*}=0$, the calculation of these values are each numerically accurate to within $\pm 0.1 \%$. It should be noted that when $0<R^{*}<r_{c}{ }^{*}$, the lower integral limit in equation (3.5) becomes negative; however, it can be easily evaluated using the fact that the stream is symmetric. In addition, because the results are normalized with respect to jet center, equation (3.5) is unity at $r=0$.

The probability distribution function, $P(r)$, initially used was based on work in Refs. [1417], in which the spatial distribution was characterized by measuring the erosion scar radius on a dye coated target. In Refs. [14-17], a larger scale blasting apparatus was characterized, and a Gamma distribution was found to fit $P(r)$ (equation (1.2)) well. However, in the present work, the nozzle was much smaller, and a Weibull distribution (shape, scale, and location parameters of $2, d / \beta$, and 0 , respectively) provided a better fit to experimentally collected values [25]:

$$
P_{2}^{W}(r)=2\left(\frac{\beta}{d}\right)^{2} r e^{-\left(\frac{\beta \cdot r}{d}\right)^{2}}
$$

or in its non-dimensional form (obtained from $\left.P(r) d r=P\left(r^{*}\right) d r^{*}\right)$ :

$$
P_{2}^{W}\left(r^{*}\right)=2 \beta^{2} r^{*} e^{-\left(\beta \cdot r^{*}\right)^{2}}
$$

where $\beta$ and $d$ are the focus coefficient and the standoff distance, respectively, as previously discussed, and the superscript $W$ and the subscript 2 indicate the Weibull distribution and its corresponding shape factor, respectively. Inserting equation (3.6b) into equation (3.5) and plotting the result against the non-dimensional radial coordinate, $r^{*}$, yielded a family of curves for various $\beta$ values (Figures 3.4-3.6, 3.8, 3.10-3.13). The $\beta$ values best characterizing the experimental data were obtained by finding a best fit of expression (3.5), using the Weibull 
distribution (equation (3.6)), to experimental data points, by adjusting $\beta$ until a maximum regression coefficient $\left(\mathrm{R}^{2}\right)$ value was reached. A standard numerical least squares method of evaluating $\beta$ could not be employed due to the complicated nature of equation (3.5) (i.e., due to a formation of a singularity during numerical evaluation, resulting from the fact that the function was only valid for $r^{*}>r_{c}{ }^{*}$ ). Average $\mathrm{R}^{2}$ values of the Weibull distribution were between 0.96 0.99 , indicating a good fit to expression (3.6).

Equation (3.6), or any $P(r)$, can be used to form an expression for the particle mass flux, $\Phi(r)$ (i.e., the mass of particles per unit time passing through a unit ring area at a given $r$, Figure 3.3) $[25]$ :

$$
\Phi(r)=\frac{\dot{M} P(r) d r}{2 \pi r d r}=\frac{\dot{M}}{\pi}\left(\frac{\beta}{d}\right)^{2} e^{-\left(\frac{\beta \cdot r}{d}\right)^{2}}
$$

where $\dot{M}$ is the mass flow rate $(\mathrm{kg} / \mathrm{s})$ through the nozzle. Normalizing to the mass flux at the center of the jet, and using $r^{*}=r / d$, this becomes [25]:

$$
\Phi^{*}\left(r^{*}\right)=\frac{\frac{\dot{M} P\left(r^{*}\right) d r^{*}}{2 \pi r^{*} d r^{*}}}{\left.\frac{\dot{M} P\left(r^{*}\right) d r^{*}}{2 \pi r^{*} d r^{*}}\right|_{r^{*}=0}}=e^{-\left(\beta \cdot r^{*}\right)^{2}}
$$

where $\Phi^{*}\left(r^{*}\right)$ is the non-dimensional normalized mass flux. 


\subsection{Results}

\subsubsection{Round Nozzles}

Figures 3.4-3.13 show the results for round nozzles for measured and fitted $M\left(R^{*}\right) / M(0)$ as a function of the non-dimensional radial distance $r^{*}$ plotted against equations (3.5) or (3.9) (see below). The corresponding measured $M\left(R^{*}\right) / M(0)$ values are recorded in Appendix $\mathrm{A}$ in Tables A-1a-A-14a.

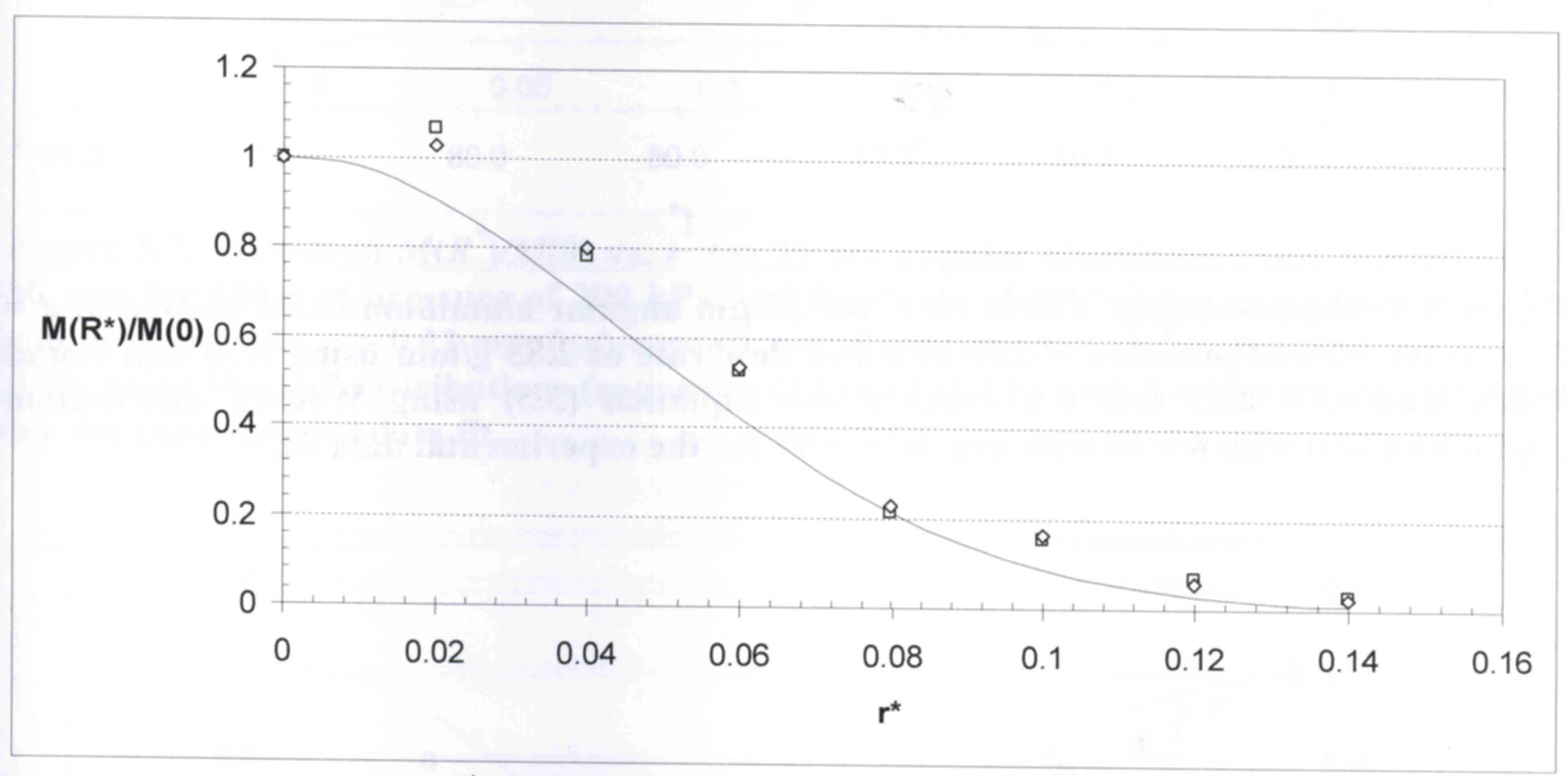

Figure 3.4: Measured $M\left(R^{*}\right) / M(0)$ vs. $r^{*}$ for $10 \mu \mathrm{m}$ angular aluminum oxide blasted at $d=$ $30 \mathrm{~mm}$ for $120 \mathrm{~s}$ at pressure of $200 \mathrm{kPa}$ and flow rate of $3.97 \mathrm{~g} / \mathrm{min}$ using $0.76 \mathrm{~mm}$ round nozzle and $r_{c}=0.6 \mathrm{~mm}$ ( $\square, \diamond-$ repeat). - Equation (3.5) using Weibull distribution (equation (3.6)) with $\beta=16$ with avg. $R^{2}=0.98$ for the experimental data fit. 


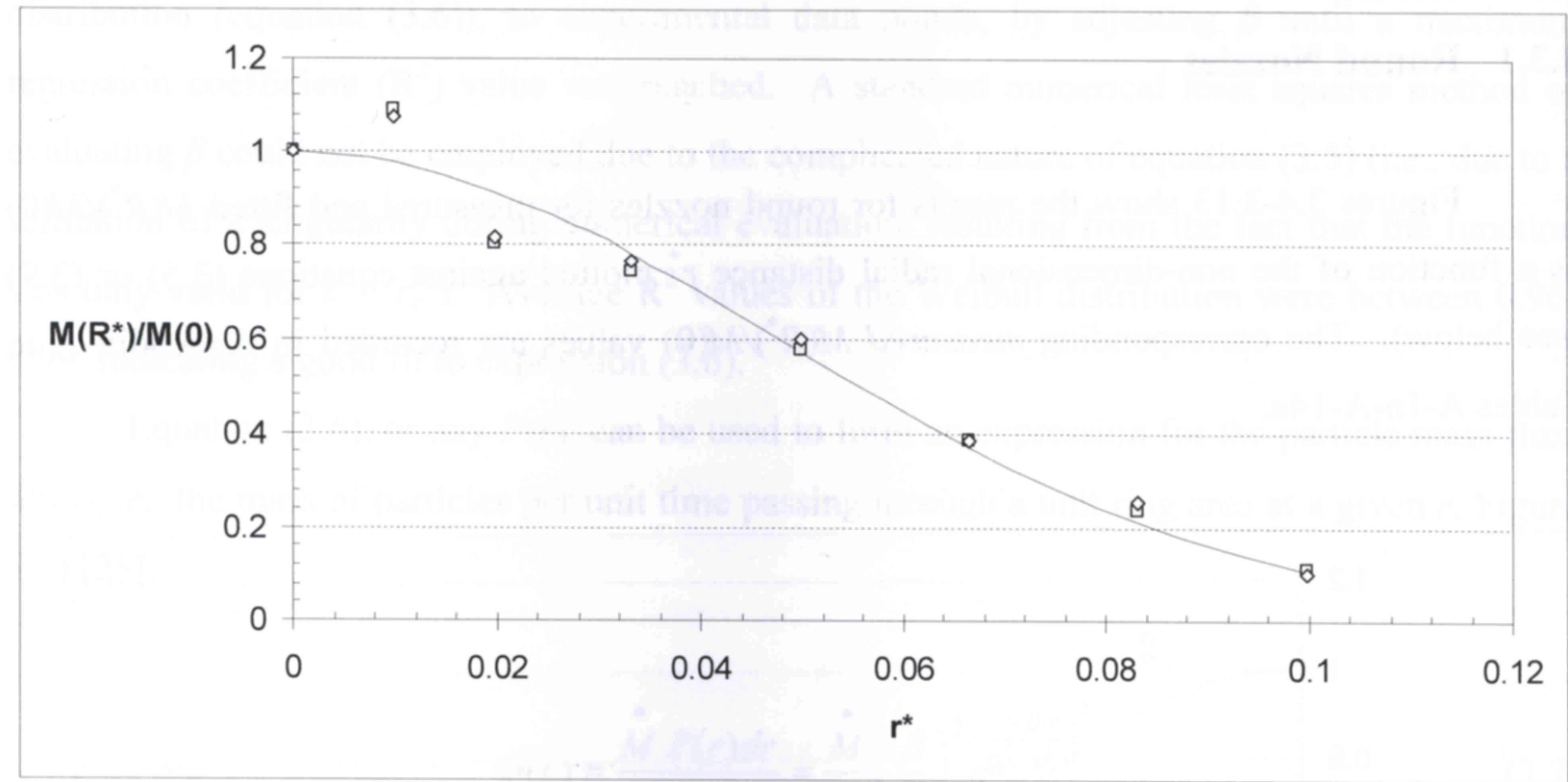

Figure 3.5: Measured $M\left(R^{*}\right) / M(0)$ vs. $r^{*}$ for $25 \mu \mathrm{m}$ angular aluminum oxide blasted at $d=$ $30 \mathrm{~mm}$ for $90 \mathrm{~s}$ at pressure of $200 \mathrm{kPa}$ and flow rate of $2.83 \mathrm{~g} / \mathrm{min}$ using $0.76 \mathrm{~mm}$ round nozzle and $r_{c}=0.23 \mathrm{~mm}(\square, \diamond$-repeat). - : Equation (3.5) using Weibull distribution (equation (3.6)) with $\beta=15$ with avg. $R^{2}=0.97$ for the experimental data fit.

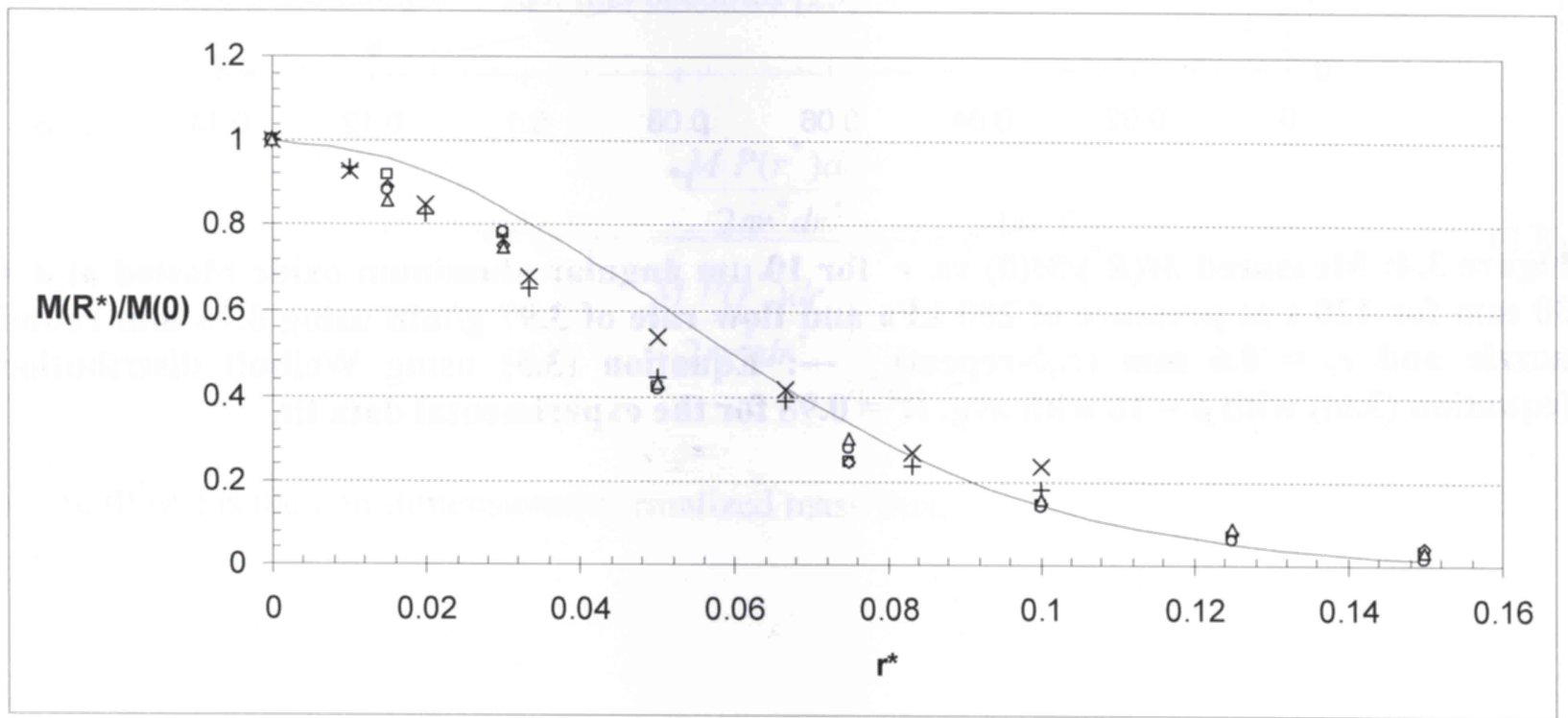

Figure 3.6: Measured $M\left(R^{*}\right) / M(0)$ vs. $r^{*}$ for $50 \mu \mathrm{m}$ silica glass beads blasted for $120 \mathrm{~s}$ at pressure of $200 \mathrm{kPa}$ using $0.76 \mathrm{~mm}$ round nozzle and $\boldsymbol{r}_{c}=0.23 \mathrm{~mm}$ ( $\square, \diamond-$ repeat: $d=20 \mathrm{~mm}$, flow rate $=1.17 \mathrm{~g} / \mathrm{min}$, avg. $\mathrm{R}^{2}=0.97 ; \mathrm{x},+-$ repeat: $d=30 \mathrm{~mm}$, flow rate $=1.17 \mathrm{~g} / \mathrm{min}$, avg. $\mathrm{R}^{2}=0.96$; $\circ, \Delta$-repeat: $d=20 \mathrm{~mm}$, flow rate $=0.59 \mathrm{~g} / \mathrm{min}$, avg. $\left.\mathrm{R}^{2}=0.97\right)$. - : Equation (3.5) using Weibull distribution (equation (3.6)) with $\beta=14$.

Fi 


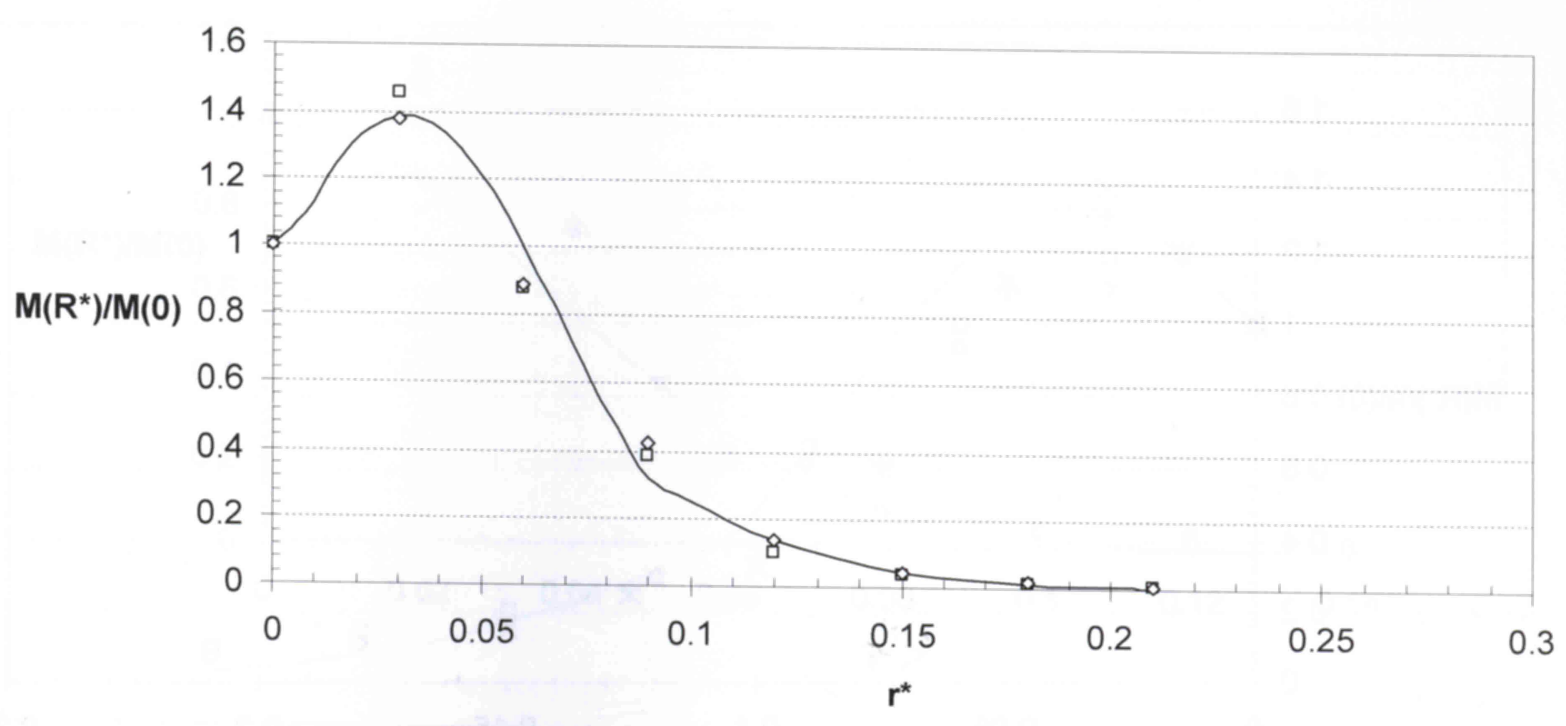

Figure 3.7: Measured $M\left(R^{*}\right) / M(0)$ vs. $r^{*}$ for $25 \mu \mathrm{m}$ angular aluminum oxide blasted at $d=$ $10 \mathrm{~mm}$ for $180 \mathrm{~s}$ at pressure of $200 \mathrm{kPa}$ and flow rate of $0.36 \mathrm{~g} / \mathrm{min}$ using $0.46 \mathrm{~mm}$ H.P. round nozzle and $r_{c}=0.23 \mathrm{~mm}(\square, \diamond-$ repeat $)$. - : Equation (3.9) $\left(F_{3}{ }^{P}=-0.025, F_{3}{ }^{S}=1.9, A=\right.$ 0.09) using Weibull distributions (equations (3.6) and (3.10)) with $\beta=12$ with avg. $R^{2}=0.99$ for the experimental data fit.

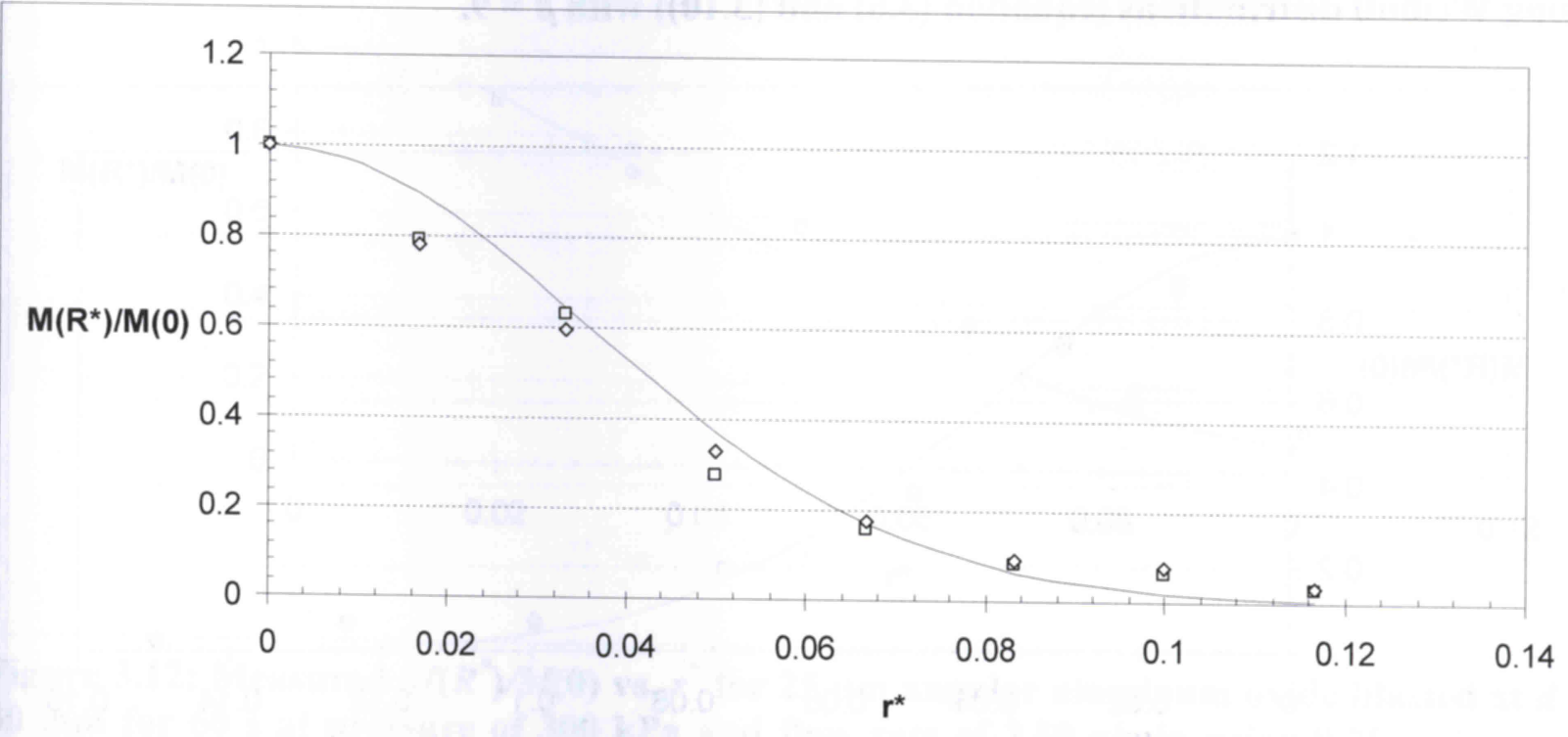

Figure 3.8: Measured $M\left(R^{*}\right) / M(0)$ vs. $r^{*}$ for $25 \mu \mathrm{m}$ angular aluminum oxide blasted at $d=$ $30 \mathrm{~mm}$ for $60 \mathrm{~s}$ at pressure of $200 \mathrm{kPa}$ and flow rate of $4.75 \mathrm{~g} / \mathrm{min}$ using $1.5 \mathrm{~mm} \mathrm{H}$.P. round nozzle and $r_{c}=0.23 \mathrm{~mm}(\square, \diamond$-repeat). —: Equation (3.5) using Weibull distribution (equation (3.6)) with $\beta=20$ with avg. $R^{2}=0.99$ for the experimental data fit. 


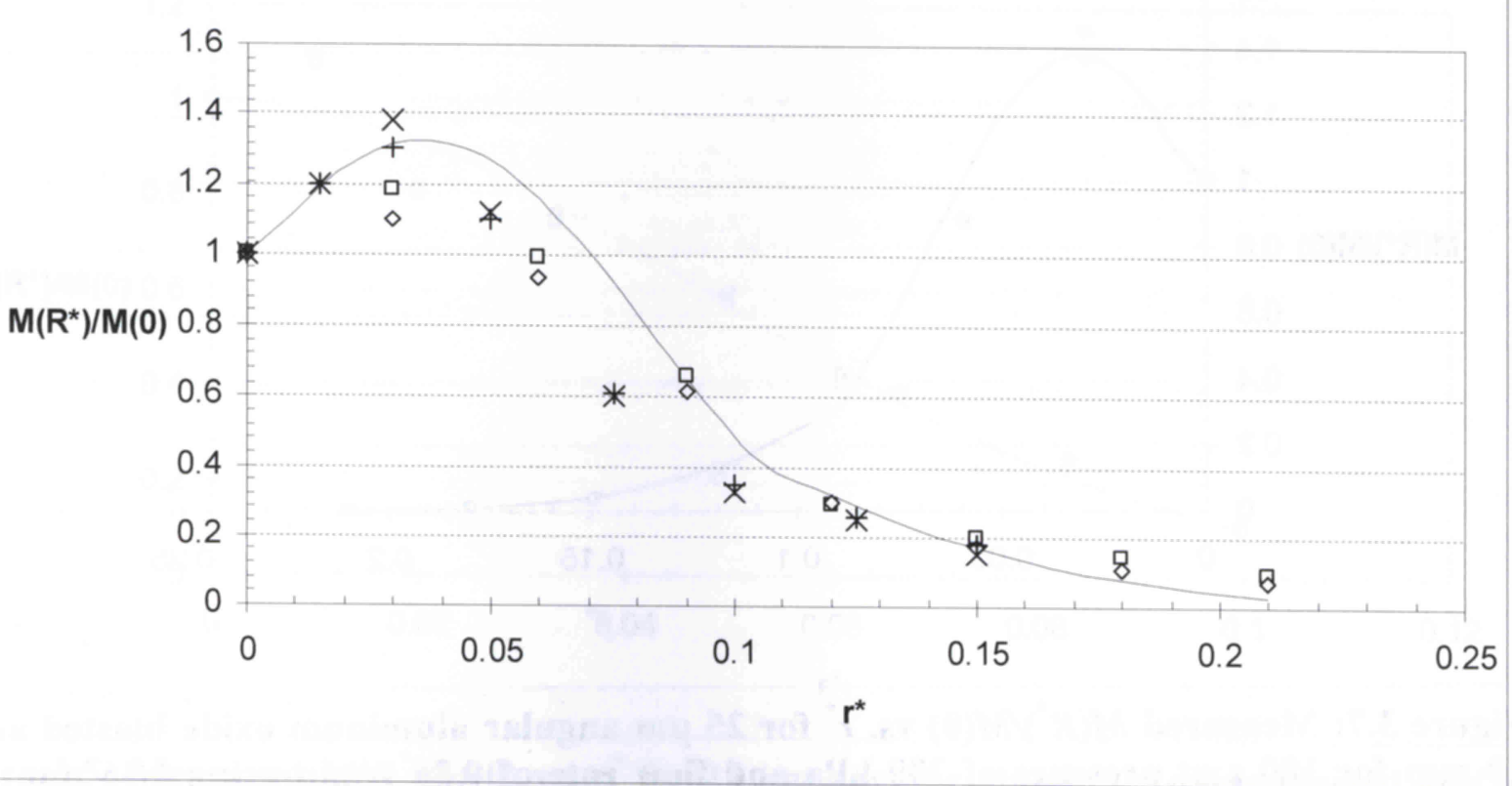

Figure 3.9: Measured $M\left(R^{*}\right) / M(0)$ vs. $r^{*}$ for $50 \mu \mathrm{m}$ silica glass beads blasted at pressure of $200 \mathrm{kPa}$ and flow rate of $0.23 \mathrm{~g} / \mathrm{min}$ using $0.46 \mathrm{~mm}$ H.P. round nozzle and $\boldsymbol{r}_{c}=0.23 \mathrm{~mm}$ (,$\diamond-$-repeat: $d=10 \mathrm{~mm}$, exposure time $=180 \mathrm{~s}$, avg. $\mathrm{R}^{2}=0.99 ; \mathbf{x},+-$ repeat: $d=20 \mathrm{~mm}$, exposure time $=240 \mathrm{~s}$, avg. $\left.\mathrm{R}^{2}=0.94\right)$. - Equation $(3.9)\left(F_{3}{ }^{P}=-0.04, F_{3}{ }^{S}=1.8, A=0.1\right)$ using Weibull distributions (equation (3.6) and (3.10)) with $\beta=9$.

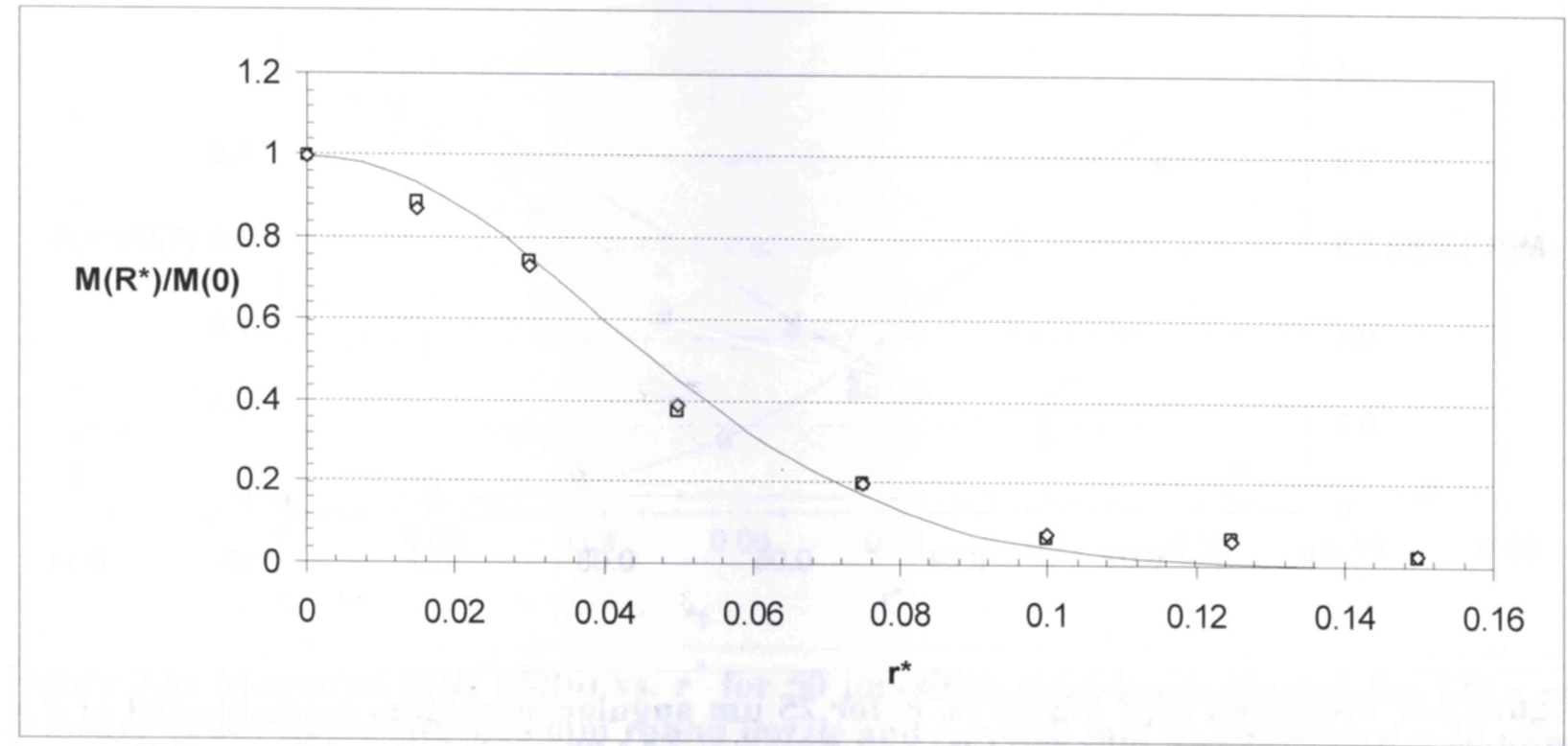

Figure 3.10: Measured $M\left(R^{*}\right) / M(0)$ vs. $r^{*}$ for $50 \mu \mathrm{m}$ silica glass beads blasted at $d=20 \mathrm{~mm}$ for $60 \mathrm{~s}$ at pressure of $200 \mathrm{kPa}$ and flow rate of $1.31 \mathrm{~g} / \mathrm{min}$ using $1.5 \mathrm{~mm} \mathrm{H.P.} \mathrm{round} \mathrm{nozzle}$ and $r_{c}=0.23 \mathrm{~mm}(\square, \diamond$-repeat). - : Equation (3.5) using Weibull distribution (equation (3.6)) with $\beta=18$ with avg. $R^{2}=0.99$ for the experimental data fit. 


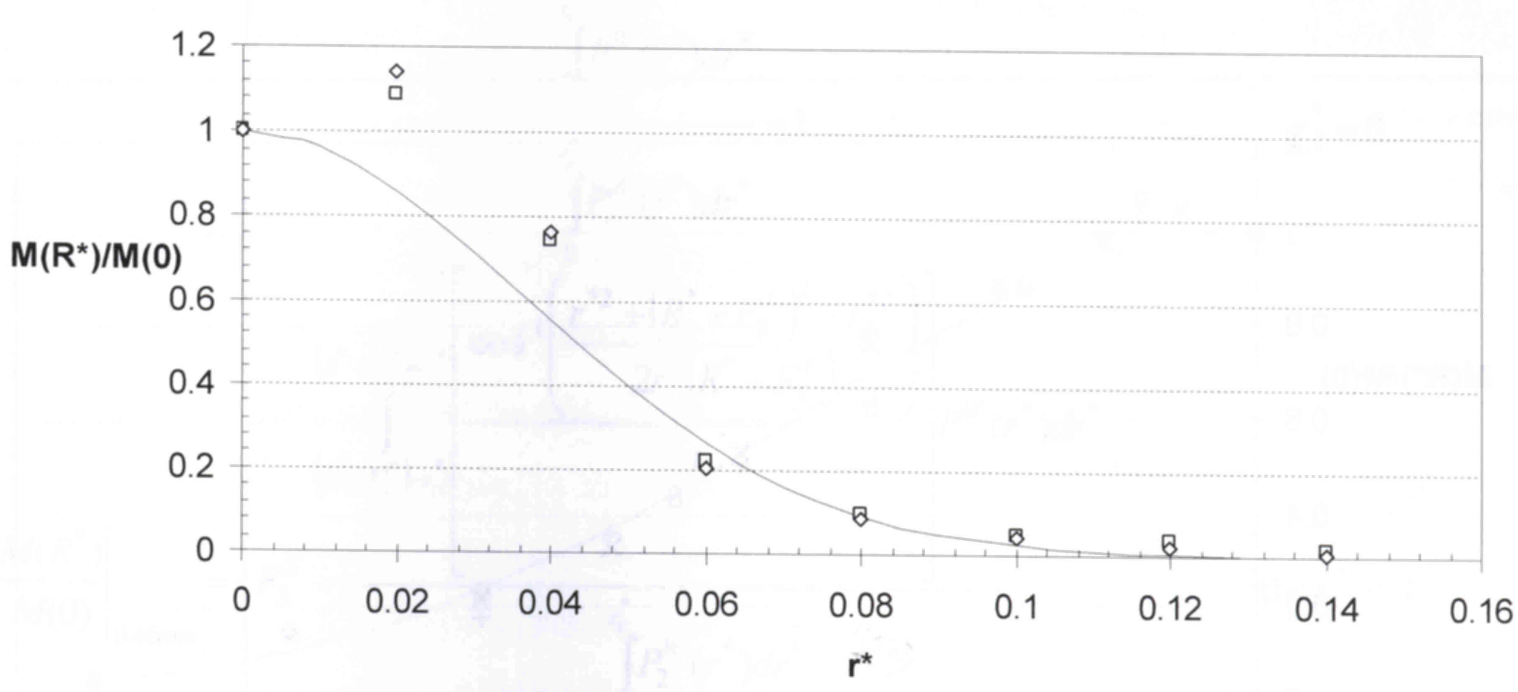

Figure 3.11: Measured $M\left(R^{*}\right) / M(0)$ vs. $r^{*}$ for $10 \mu \mathrm{m}$ angular aluminum oxide blasted at $d=$ $30 \mathrm{~mm}$ for $60 \mathrm{~s}$ at pressure of $300 \mathrm{kPa}$ and flow rate of $4.20 \mathrm{~g} / \mathrm{min}$ using $0.76 \mathrm{~mm}$ round nozzle and $r_{c}=0.6 \mathrm{~mm}$ ( $\square, \diamond-$-repeat). - Equation (3.5) using Weibull distribution (equation (3.6)) with $\beta=20$ with avg. $R^{2}=0.96$ for the experimental data fit.

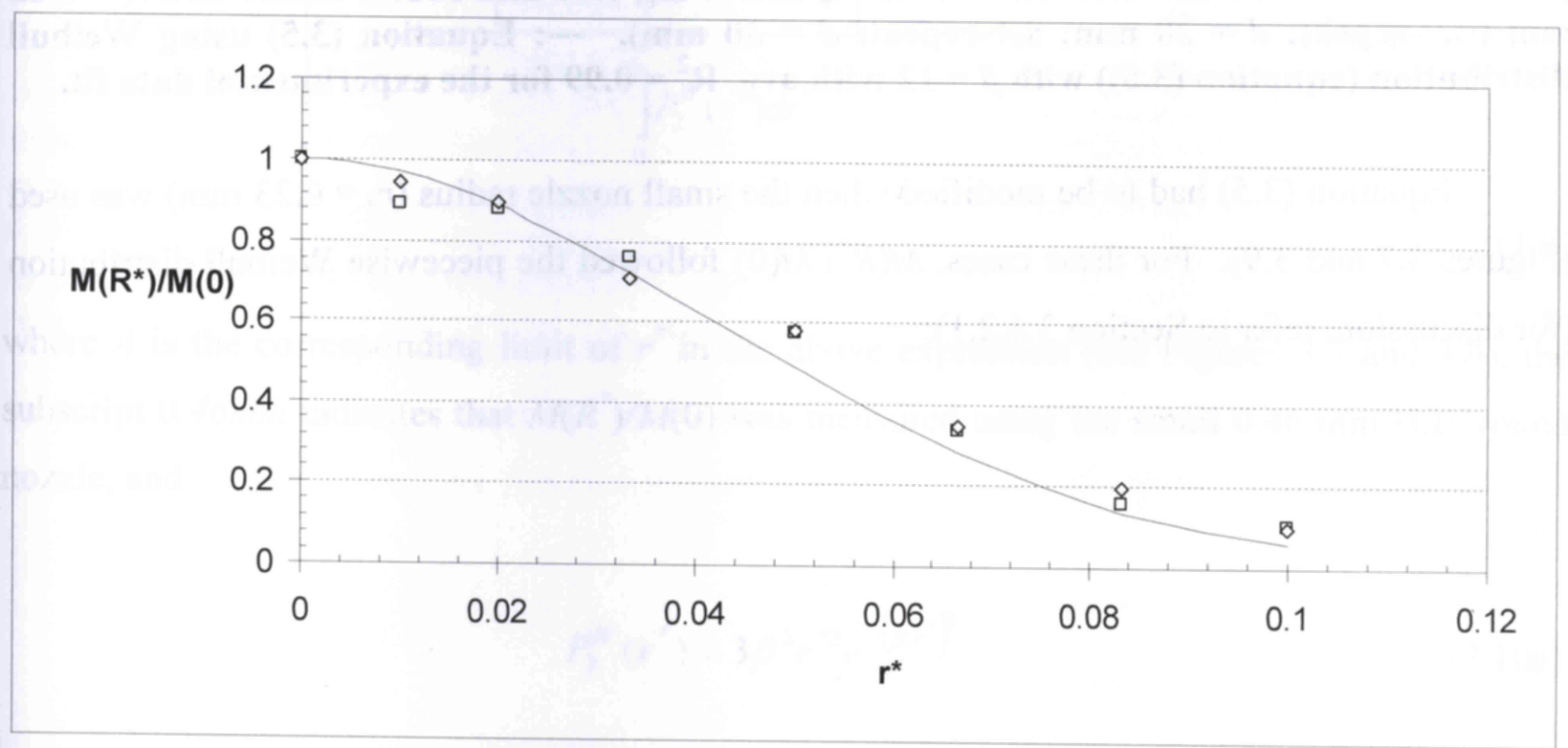

Figure 3.12: Measured $M\left(R^{*}\right) / M(0)$ vs. $r^{*}$ for $25 \mu \mathrm{m}$ angular aluminum oxide blasted at $d=$ $30 \mathrm{~mm}$ for $60 \mathrm{~s}$ at pressure of $300 \mathrm{kPa}$ and flow rate of $3.10 \mathrm{~g} / \mathrm{min}$ using $0.76 \mathrm{~mm}$ round nozzle and $r_{c}=0.23 \mathrm{~mm}$ ( $\square, \diamond$-repeat). - Equation (3.5) using Weibull distribution (equation (3.6)) with $\beta=17$ with avg. $R^{2}=0.99$ for the experimental data fit. 


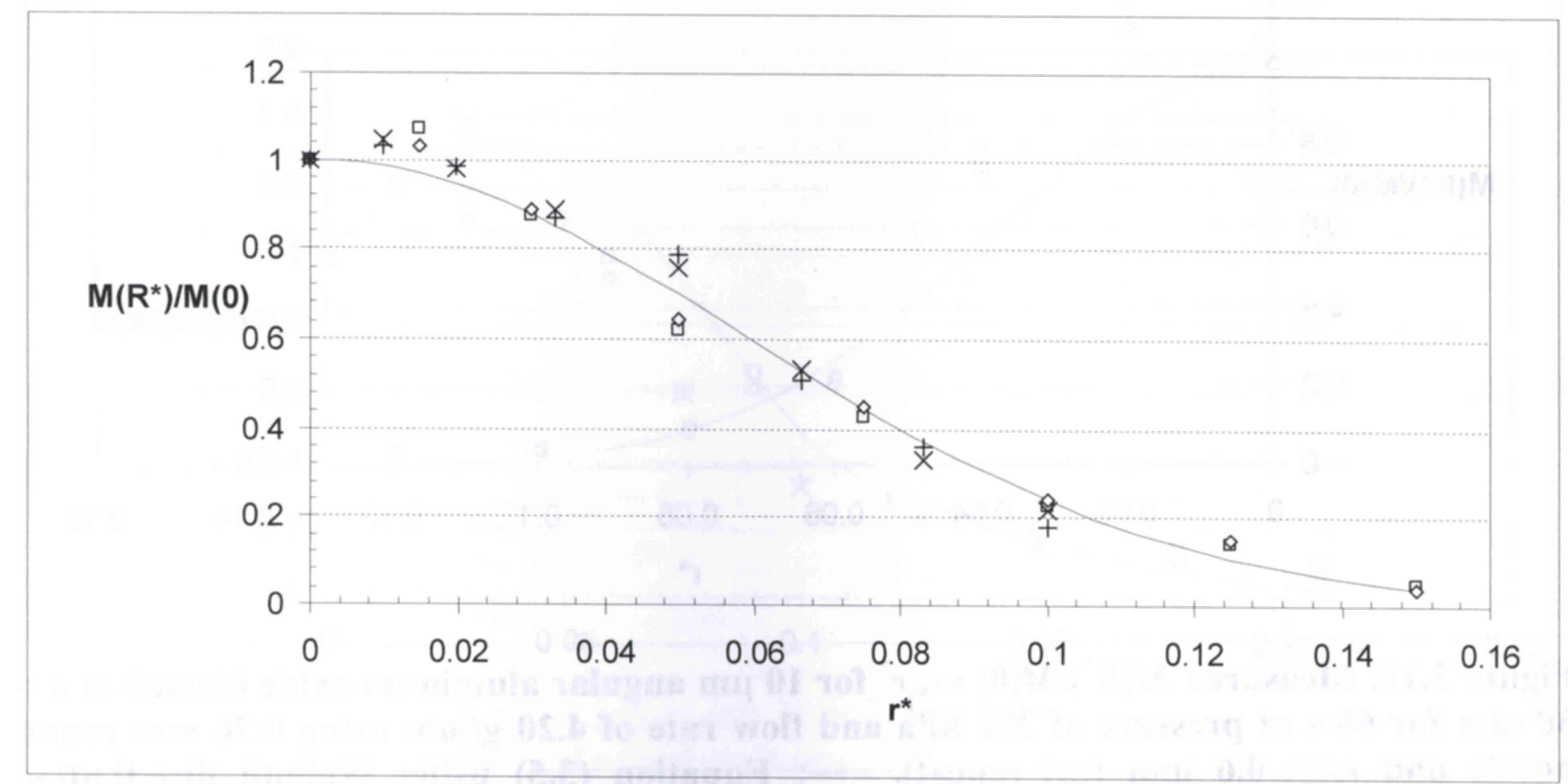

Figure 3.13: Measured $M\left(R^{*}\right) / M(0)$ vs. $r^{*}$ for $50 \mu \mathrm{m}$ silica glass beads blasted for $90 \mathrm{~s}$ at pressure of $300 \mathrm{kPa}$ and flow rate of $1.34 \mathrm{~g} / \mathrm{min}$ using $0.76 \mathrm{~mm}$ round nozzle and $\boldsymbol{r}_{c}=0.23$ $\mathrm{mm}$ ( $\square, \diamond$-repeat: $d=20 \mathrm{~mm}$; $x,+-$ repeat: $d=30 \mathrm{~mm}$ ). - : Equation (3.5) using Weibull distribution (equation (3.6)) with $\beta=12$ with avg. $R^{2}=0.99$ for the experimental data fit.

Equation (3.5) had to be modified when the small nozzle radius $\left(r_{n}=0.23 \mathrm{~mm}\right)$ was used (Figures 3.7 and 3.9). For these cases, $M\left(R^{*}\right) / M(0)$ followed the piecewise Weibull distribution (for discussion, refer to Section 3.4.2.1): 


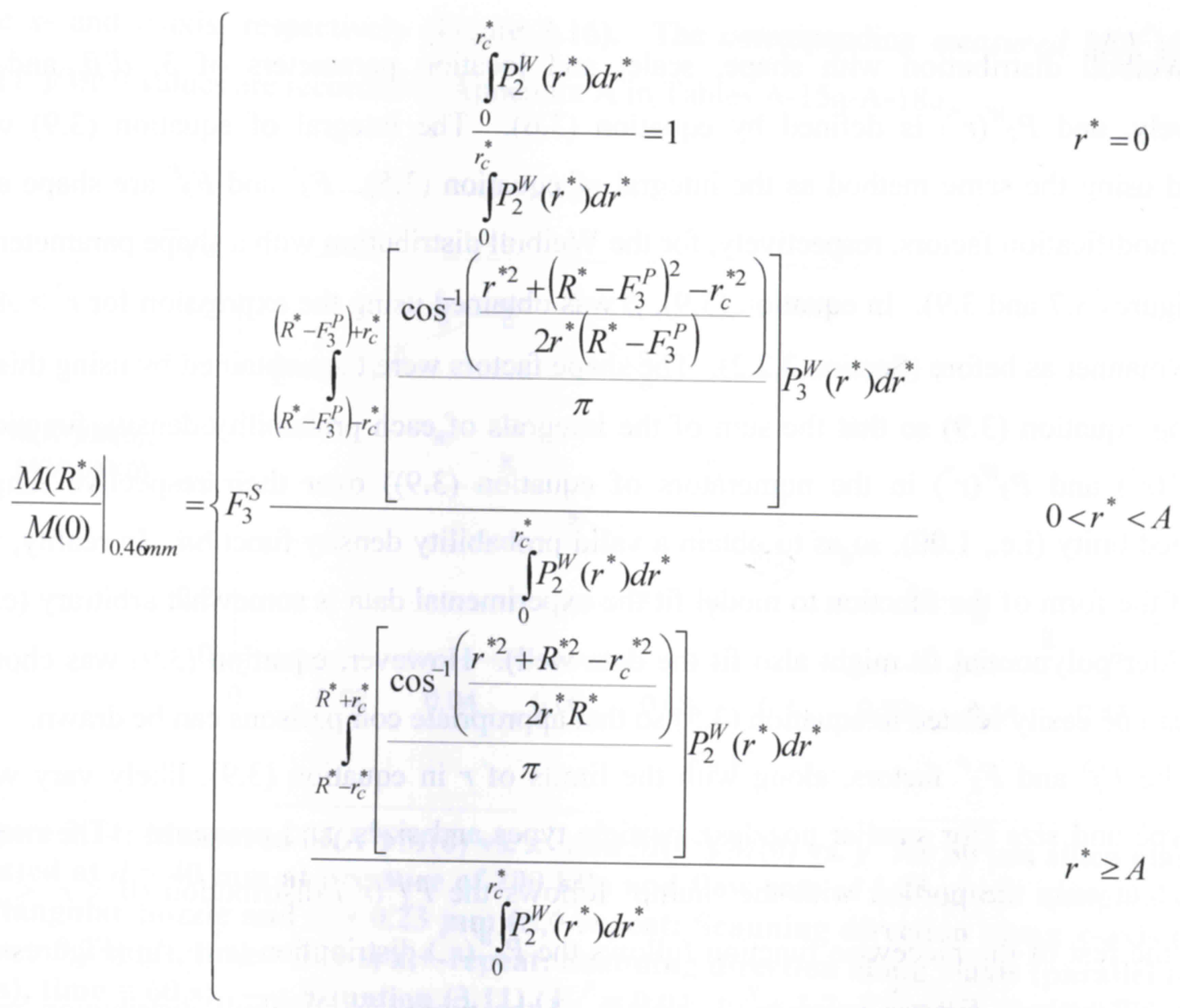

where $A$ is the corresponding limit of $r^{*}$ in the above expression (see Figures 3.7 and 3.9), the subscript $0.46 \mathrm{~mm}$ indicates that $M\left(R^{*}\right) / M(0)$ was measured using the small $0.46 \mathrm{~mm}$ H.P. round nozzle, and

$$
P_{3}^{W}\left(r^{*}\right)=3 \beta^{3} r^{* 2} e^{-\left(\beta \cdot r^{*}\right)^{3}}
$$

or in its dimensional form,

$$
P_{3}^{W}(r)=3\left(\frac{\beta}{d}\right)^{3} r^{2} e^{-\left(\frac{\beta \cdot r}{d}\right)^{3}}
$$


is the Weibull distribution with shape, scale, and location parameters of $3, d / \beta$, and 0 , respectively, and $P_{2}{ }^{W}\left(r^{*}\right)$ is defined by equation (3.6). The integral of equation (3.9) was evaluated using the same method as the integral of equation (3.5). $F_{3}{ }^{S}$ and $F_{3}{ }^{P}$ are shape and position modification factors, respectively, for the Weibull distribution with a shape parameter of 3 (see Figures 3.7 and 3.9). In equation (3.9), $\beta$ was obtained using the expression for $r^{*}>A$ in the same manner as before (Section 3.2.2). The shape factors were then obtained by using this $\beta$ and fitting equation (3.9) so that the sum of the integrals of each probability density functions (i.e., $P_{2}{ }^{W}\left(r^{*}\right)$ and $P_{3}{ }^{W}\left(r^{*}\right)$ in the numerators of equation (3.9)) over their respective ranges approached unity (i.e., 1.00), so as to obtain a valid probability density function. In reality, the choice of the form of the function to model fit the experimental data is somewhat arbitrary (e.g., a high order polynomial fit might also fit the data well). However, equation (3.9) was chosen since it can be easily related to equation (3.5) so that appropriate comparisons can be drawn.

The $F_{3}{ }^{S}$ and $F_{3}{ }^{P}$ factors, along with the limits of $r$ in equation (3.9), likely vary with nozzle type and size (for smaller nozzles), particle types and sizes, and pressures. The model assumes that only the portion with the 'hump' follows the $P_{3}{ }^{W}\left(r^{*}\right)$ distribution $\left(0<r^{*}<\mathrm{A}\right)$, whereas the rest of the piecewise function follows the $P_{2}{ }^{W}\left(r^{*}\right)$ distribution $\left(r^{*}>\right.$ A) (Figures 3.7 and 3.9). For these figures, average $\mathrm{R}^{2}$ values of the piecewise Weibull distribution were between 0.94-0.99, indicating a good fit to equations (3.6) and (3.10). The expression for the flux at a specific coordinate $r^{*}$ can be obtained by using the same procedure as outlined by equations (3.7)-(3.8) using a probability density function from the numerator of equation (3.9) of the respective range where the coordinate of interest is located; i.e., using equations (3.6) or (3.10).

\subsubsection{Rectangular Nozzles}

Figures 3.14-3.15 show the results for rectangular nozzles for measured and fitted $M\left(X^{*}\right) / M(0)$ and $M\left(Y^{*}\right) / M(0)$, when measuring along the $\mathrm{x}$-axis (parallel to shorter side of inner opening of the nozzle, $w$ ) and y-axis (parallel to longer side of inner opening of the nozzle, $l$ ) as a function of normalized coordinate distance $x^{*}=x / d$ and $\mathrm{y}^{*}=y / d$ (Figure 3.16), respectively, plotted against equations (3.11) or (3.16) (see below). Again, $X^{*}=X / d$ and $Y^{*}=Y / d$ are the nondimensional distances from the jet center to the center of the collection cylinder measured along 
the $x$ - and $y$-axis, respectively (Figure 3.16). The corresponding measured $M\left(X^{*}\right) / M(0)$ and $M\left(Y^{*}\right) / M(0)$ values are recorded in Appendix A in Tables A-15a-A-18a.

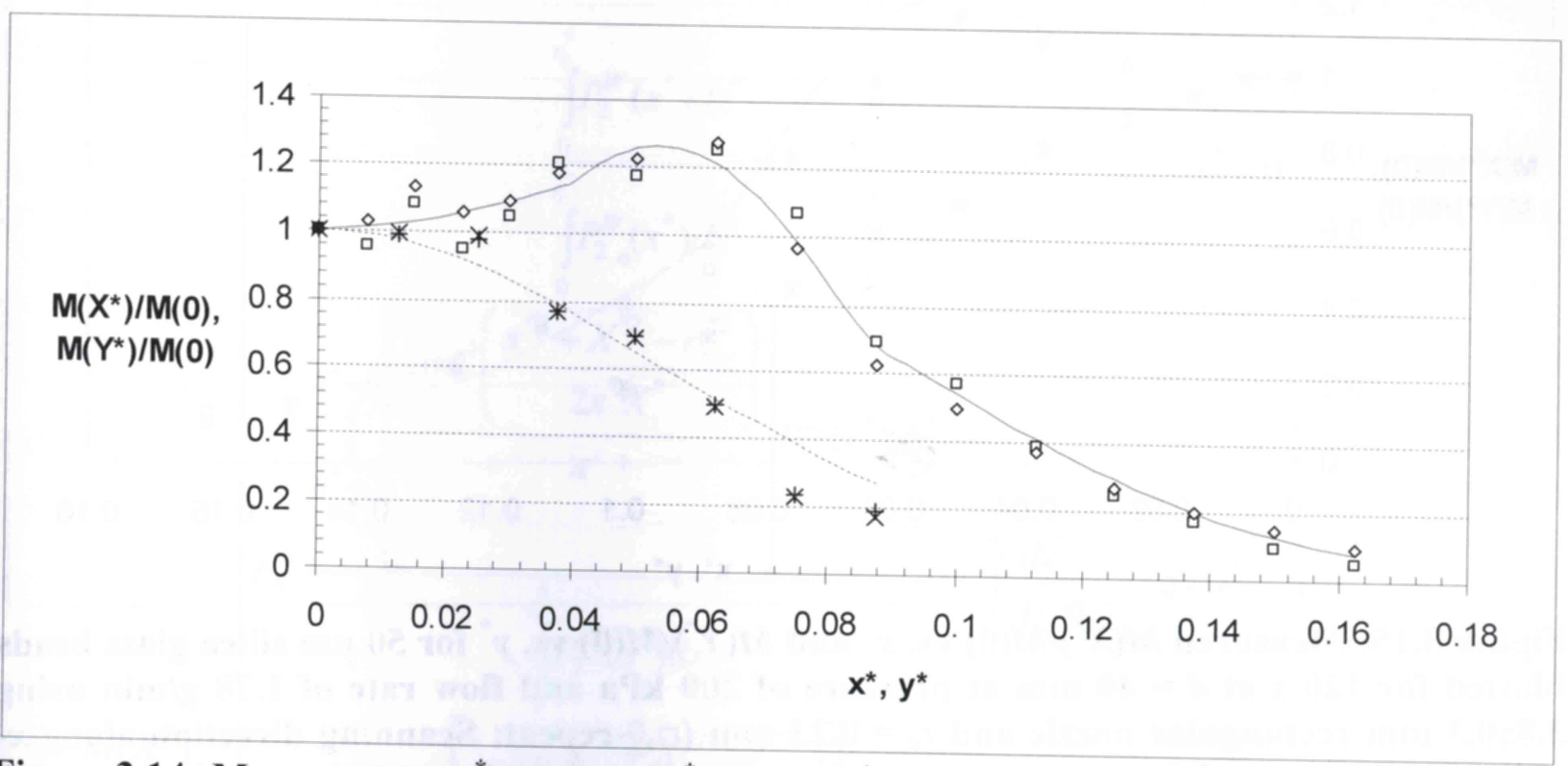

Figure 3.14: Measured $M\left(X^{*}\right) / M(0)$ vs. $x^{*}$ and $M\left(Y^{*}\right) / M(0)$ vs. $y^{*}$ for $50 \mu \mathrm{m}$ silica glass beads blasted at $d=40 \mathrm{~mm}$ at pressure of $200 \mathrm{kPa}$ and flow rate of $1.21 \mathrm{~g} / \mathrm{min}$ using $3.2 \times 0.2 \mathrm{~mm}$ rectangular nozzle and $\boldsymbol{r}_{c}=\mathbf{0 . 2 3} \mathbf{\mathrm { mm }}(\square, \diamond$-repeat: Scanning direction along $x$-axis (parallel to $w=0.2 \mathrm{~mm}$ ), time $=120 \mathrm{~s} ; \mathrm{x},+$-repeat: Scanning direction along $y$-axis (parallel to $l=3.2$ $\mathrm{mm})$, time $=60 \mathrm{~s})$. - : Equation (3.11) $\left(F_{2}{ }^{P}=0.04, F_{3}{ }^{S}=1.7, F_{4}{ }^{S}=0.3, A=0.0375\right.$, and $B=$ 0.0875 ) using Weibull distributions (equations (3.12), (3.13) and (3.14)) with $\beta=13$ with avg. $\mathbf{R}^{2}=0.98$ for the experimental data fit. ----: Equation (3.16) using Weibull distribution (equation (3.17)) with $\beta=13$ with avg. $R^{2}=0.98$ for the experimental data fit. 


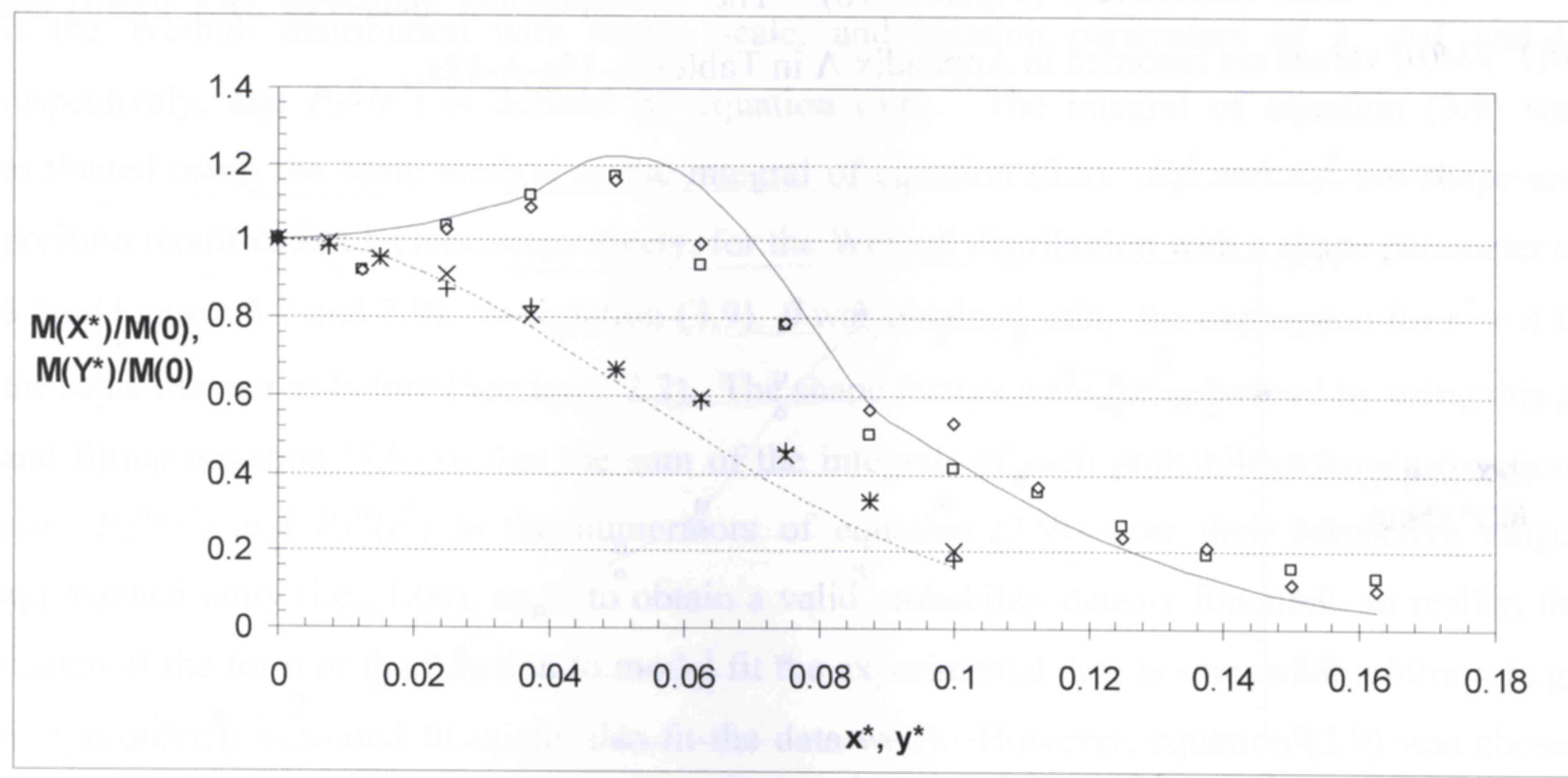

Figure 3.15: Measured $M\left(X^{*}\right) / M(0)$ vs. $x^{*}$ and $M\left(Y^{*}\right) / M(0)$ vs. $y^{*}$ for $50 \mu \mathrm{m}$ silica glass beads blasted for $120 \mathrm{~s}$ at $d=40 \mathrm{~mm}$ at pressure of $200 \mathrm{kPa}$ and flow rate of $1.78 \mathrm{~g} / \mathrm{min}$ using $3.8 \times 0.3 \mathrm{~mm}$ rectangular nozzle and $r_{c}=0.23 \mathrm{~mm}(\square, \diamond$-repeat: Scanning direction along $x$ axis (parallel to $w=0.3 \mathbf{m m}$ ); $x$,+-repeat: Scanning direction along $y$-axis (parallel to $l=3.8$ $\mathrm{mm}))$. - Equation (3.11) $\left(F_{2}{ }^{P}=0.0325, F_{3}{ }^{S}=1.65, F_{4}{ }^{S}=0.25, A=0.0375\right.$, and $\left.B=0.0875\right)$ using Weibull distributions (equations (3.12), (3.13) and (3.14)) with $\beta=13.5$ with avg. $R^{2}=$ 0.98 for the experimental data fit. ----: Equation (3.16) using Weibull distribution (equation (3.17)) with $\beta=13.5$ with avg. $R^{2}=0.99$ for the experimental data fit.

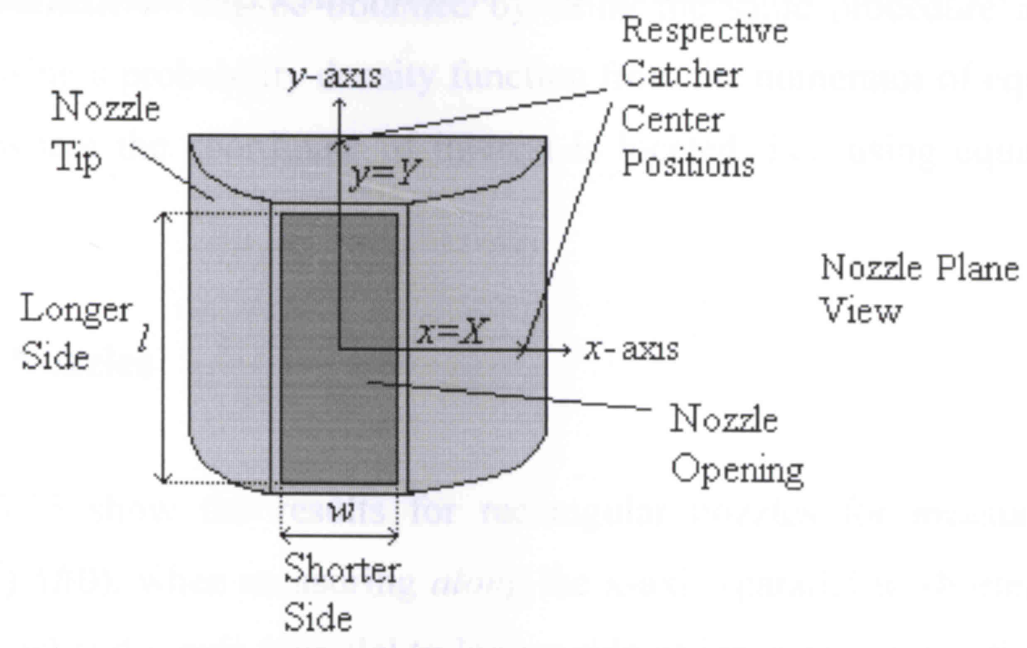

Figure 3.16: Nozzle plane view of the inner opening of a rectangular nozzle (shorter side, $w$, and longer side, $l$ ), and the $x$ - and $y$-axes, along with respective center line distances from the jet center to center of collection cylinder, $X$ and $Y$ (analogous to $R$; see Figure 3.3). 


\subsubsection{2):}

$M\left(X^{*}\right) / M(0)$ followed the piecewise Weibull distribution (for discussion, refer to Section

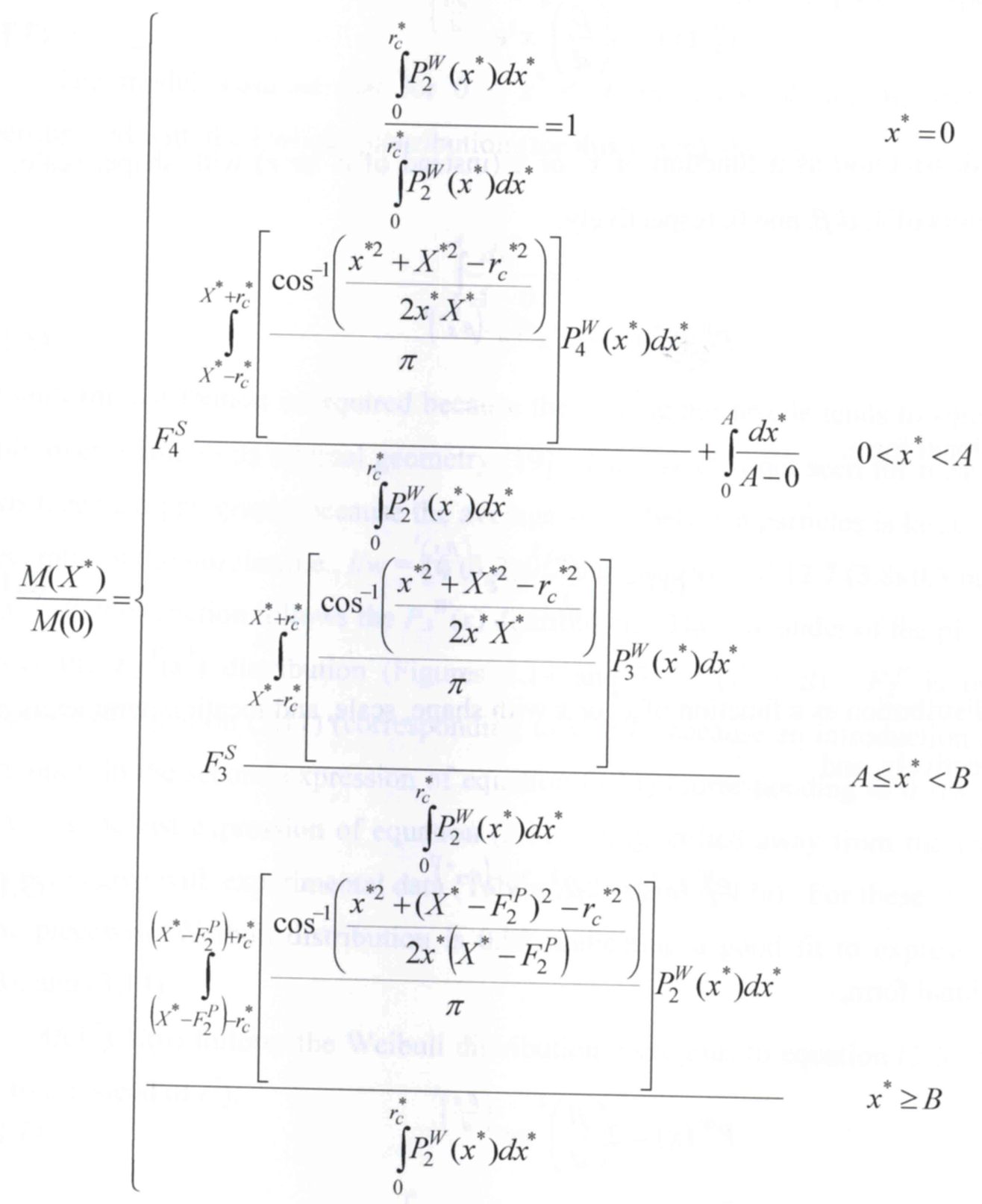

where $A$ and $B$ (see Figures 3.14-3.15) are the corresponding limits of $x^{*}$ (instead of $r^{*}$ ) in the above expression,

$$
P_{4}^{W}\left(x^{*}\right)=4 \beta^{4} x^{* 3} e^{-\left(\beta \cdot x^{*}\right)^{4}}
$$


or in its dimensional form,

$$
P_{4}^{W}(x)=4\left(\frac{\beta}{d}\right)^{4} x^{3} e^{-\left(\frac{\beta \cdot x}{d}\right)^{4}}
$$

is the Weibull distribution as a function of $x^{*}$ or $x$ (instead of $r^{*}$ or $r$ ) with shape, scale, and location parameters of $4, d / \beta$, and 0 , respectively,

$$
P_{3}^{W}\left(x^{*}\right)=3 \beta^{3} x^{* 2} e^{-\left(\beta \cdot x^{*}\right)^{3}}
$$

or in its dimensional form,

$$
P_{3}^{W}(x)=3\left(\frac{\beta}{d}\right)^{3} x^{2} e^{-\left(\frac{\beta \cdot x}{d}\right)^{3}}
$$

is the Weibull distribution as a function of $x^{*}$ or $x$ with shape, scale, and location parameters of 3 , $d / \beta$, and 0 , respectively, and

$$
P_{2}^{W}\left(x^{*}\right)=2 \beta^{2} x^{*} e^{-\left(\beta \cdot x^{*}\right)^{2}}
$$

or in its dimensional form,

$$
P_{2}^{W}(x)=2\left(\frac{\beta}{d}\right)^{2} x e^{-\left(\frac{\beta \cdot x}{d}\right)^{2}}
$$

is the Weibull distribution as a function of $x^{*}$ or $x$ with shape, scale, and location parameters of 2 , $d / \beta$, and 0. Equations (3.12b), (3.13b), and (3.14b) are applied to equation (3.11) in the same manner as equation (3.6b) to equation (3.5). The integral of equation (3.11) is evaluated with the same method as the integral of equation (3.5). $F_{3}{ }^{S}$ and $F_{4}{ }^{S}$ are shape modification factors for the Weibull distribution with shape parameters of 3 and 4 , respectively, and $F_{2}{ }^{P}$ is the position modification factor for the Weibull distribution with a shape parameter of 2 (see Figures 3.14- 
3.15). The $\beta$ and modification factors are obtained in the same manner as for equation (3.9) as explained in Section 3.3.1 (i.e., using the definition of a valid probability distribution function). The limits of $x^{*}$ in equation (3.11) likely vary with other smaller $w$ 's, particle types and sizes, and pressures.

The model assumes that for $0<x^{*}<A$, the data follows the $P_{4}{ }^{W}\left(x^{*}\right)$ distribution superimposed with the Uniform distribution (for this range), defined as

$$
\int_{0}^{A} \frac{d x^{*}}{A-0}=1
$$

The uniform distribution is required because the rectangular nozzle tends to spread the particles evenly over $w$ due to its internal geometry [19]. This effect is not seen for measurements along $\mathrm{y}$-axis (see next paragraph) because the average space between particles is larger due to the high aspect ratio of the nozzles, i.e., $l / w=16(3.2 \times 0.2 \mathrm{~mm}$ nozzle $)$ and 12.7 (3.8x0.3 mm nozzle). For $A \leq x^{*}<B$, the function follows the $P_{3}{ }^{W}(x)$ distribution. The remainder of the piecewise function follows the $P_{2}{ }^{W}\left(x^{*}\right)$ distribution (Figures 3.14 and 3.15) $\left(x^{*}>B\right) . F_{2}{ }^{P}$ is used in the last expression of equation (3.11) (corresponding to $x^{*}>B$ ) because an introduction of the Uniform distribution in the second expression of equation (3.11) (corresponding to $0<x^{*}<A$ ) results in $P_{2}{ }^{W}\left(x^{*}\right)$ in the last expression of equation (3.11) being shifted away from the jet center, which appears to agree with experimental data (Tables A-15a and A-17a). For these cases, the $\mathrm{R}^{2}$ value of the piecewise Weibull distribution is 0.98 , indicating a good fit to expressions (3.12) and (3.13), and (3.14).

$M\left(Y^{*}\right) / M(0)$ follows the Weibull distribution analogous to equation (3.5) (however, here, $y^{*}$ is used instead of $r^{*}$ ):

$$
\frac{M\left(Y^{*}\right)}{M(0)}=\frac{\int_{Y^{*}-r_{c}^{*}}^{Y^{*}+r_{c}^{*}}\left[\frac{\cos ^{-1}\left(\frac{y^{* 2}+Y^{* 2}-r_{c}^{* 2}}{2 y^{*} Y^{*}}\right)}{\pi}\right] P_{2}^{W}\left(y^{*}\right) d y^{*}}{\int_{0}^{r_{c}^{*}} P_{2}^{W}\left(y^{*}\right) d y^{*}}
$$


where,

$$
P_{2}^{W}\left(y^{*}\right)=2 \beta^{2} y^{*} e^{-\left(\beta \cdot y^{*}\right)^{2}}
$$

or in its dimensional form,

$$
P_{2}^{W}(y)=2\left(\frac{\beta}{d}\right)^{2} y e^{-\left(\frac{\beta \cdot y}{d}\right)^{2}}
$$

which is the Weibull distribution as a function of $y^{*}$ or $y$ with shape, scale, and location parameters of $2, d / \beta$, and 0 , respectively. For these cases, average $\mathrm{R}^{2}$ values of the Weibull distribution were between $0.98-0.99$, indicating a good fit to expression (3.17).

The expression for the flux along the $\mathrm{x}$ - or $\mathrm{y}$-axis (i.e., along the center line), at a coordinate $x^{*}$ or $y^{*}$ can be obtained by using an analogous procedure as outlined by equations (3.7)-(3.8), using a probability density function from the numerator of equation (3.11) of the respective range where the coordinate of interest is located, or equation (3.16); i.e., using equation (3.12), (3.13) or (3.14), or using equation (3.17), respectively. 


\subsection{Discussion of Results}

The particle spatial distribution experiments and corresponding focus coefficients $(\beta$ values) are summarized in Table 3.1. 


\begin{tabular}{|c|c|c|c|c|c|c|c|c|c|c|c|c|}
\hline 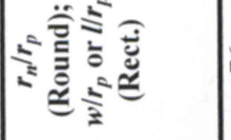 & $\stackrel{2}{2}$ & ¿ें & $\stackrel{\text { In }}{\varrho}$ & $\stackrel{\vec{\infty}}{\infty}$ & 8 & ๙ু & ळি & $\therefore$ & ¿े. & $\stackrel{\sim}{\Perp}$ & $\begin{array}{l}\text { J } \\
-\end{array}$ & $\begin{array}{l}\stackrel{2}{2} \\
6\end{array}$ \\
\hline 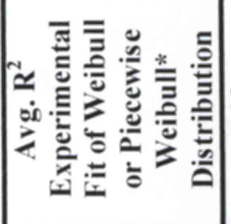 & ڤ̊. & $\hat{\sigma}$ & 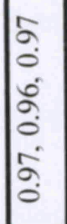 & 卷 & ठे & 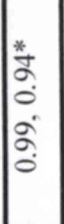 & बे & $\stackrel{\circ}{\circ}$ & ठे & बे & $\begin{array}{l}\infty \\
2 \\
0 \\
* \\
\infty \\
\infty \\
0 \\
0\end{array}$ & $\begin{array}{l}2 \\
0 \\
0 \\
\infty \\
\vdots \\
0\end{array}$ \\
\hline$\infty$ & 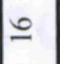 & $\because$ & \pm & $\simeq$ & సి & a & $\infty$ & సి & $=$ & $\simeq$ & $\cong$ & $\mid \begin{array}{l}n \\
m \\
m\end{array}$ \\
\hline 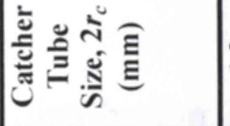 & $\stackrel{-}{=}$ & 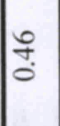 & 我 & to & to & 乐. & $\stackrel{0}{\circ}$ & 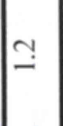 & $\mid$ & 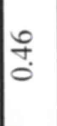 & ثீ. & 웅. \\
\hline 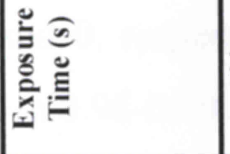 & $\beth$ & 8 & ฏิ & $\nsubseteq$ & 8 & $\begin{array}{l}\stackrel{8}{N} \\
\text { @ } \\
\stackrel{\infty}{-}\end{array}$ & 8 & 8 & 8 & 8 & בิ & సิ \\
\hline 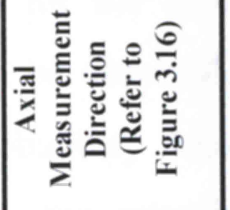 & $\overleftrightarrow{z}$ & $\ll$ & $\overleftarrow{z}$ & $\ll$ & $\ll$ & $\ll$ & $\ll$ & $\ll$ & $\leqslant$ & $\leqslant$ & $\begin{array}{l}\frac{n}{x} \\
\dot{y} \\
\dot{y} \\
\dot{x}\end{array}$ & $\mid \begin{array}{l}\frac{n}{2} \\
\vdots \\
\vdots \\
\dot{2} \\
\dot{x} \\
\dot{x}\end{array}$ \\
\hline 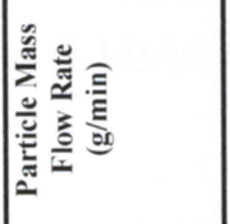 & बे & $\stackrel{\infty}{\stackrel{\infty}{i}}$ & $\begin{array}{l}n \\
0 \\
0 \\
= \\
= \\
= \\
=\end{array}$ & ?̊. & 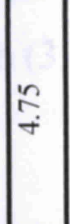 & $\stackrel{\leftrightarrow}{\circ}$ & $\stackrel{m}{\longrightarrow}$ & $\underset{\text { †ิ }}{+}$ & $\frac{\circ}{m}$ & $\stackrel{m}{m}$ & $\bar{\Upsilon}$ & $\stackrel{\infty}{\stackrel{\infty}{+}}$ \\
\hline 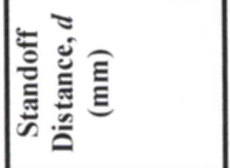 & 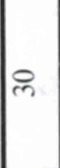 & లి & $\begin{array}{l}\text { तิ } \\
\text { हिं } \\
\stackrel{\text { ते }}{ }\end{array}$ & $\varrho$ & ల & $\begin{array}{l}\text { กิ } \\
\varrho\end{array}$ & กิ & pr & 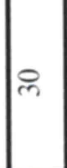 & $\begin{array}{l}\text { ते } \\
\stackrel{\text { ते }}{ }\end{array}$ & F & f \\
\hline 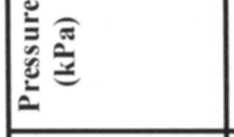 & ஓ్సి & సి & సి & స్ & ఫે & ¿ి & ‡్సి & ৪্ল & ఠ్లి & ঠ్లি & స్ & సి \\
\hline 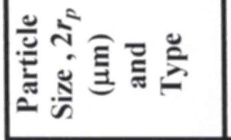 & $\begin{array}{c}0 \\
\stackrel{1}{<} \\
\stackrel{1}{<} \\
-\end{array}$ & 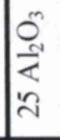 & $\begin{array}{l}0 \\
0 \\
\text { S } \\
\text { in }\end{array}$ & 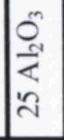 & 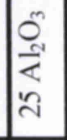 & $\begin{array}{l}0 \\
0 \\
0 \\
i n \\
i n\end{array}$ & 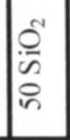 & 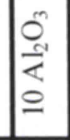 & 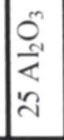 & $\begin{array}{l}0 \\
\frac{0}{n} \\
i n\end{array}$ & $\begin{array}{l}0 \\
\text { in } \\
i n\end{array}$ & $\begin{array}{l}0 \\
0 \\
\text { in } \\
\text { in }\end{array}$ \\
\hline 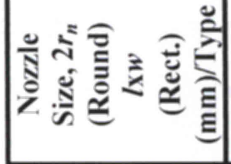 & $\frac{0}{2}$ & : & $\stackrel{\circ}{\circ}$ & 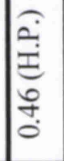 & 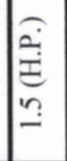 & 琶 & 命 & : & ז̊ & $\stackrel{0}{\circ}$ & 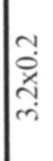 & $\begin{array}{l}n \\
\dot{0} \\
\dot{\alpha} \\
\dot{\alpha}\end{array}$ \\
\hline 然 & $\vec{r}$ & m & மே. & $\hat{m}$ & $\begin{array}{l}\infty \\
\dot{n}\end{array}$ & ले & $\frac{\varrho}{\dot{r}}$ & $\bar{m}$ & $\stackrel{\sim}{m}$ & $\frac{m}{m}$ & $\frac{ \pm}{m}$ & $\stackrel{\sim}{m}$ \\
\hline
\end{tabular}




\subsubsection{Effect of Particle Size and Type}

From Figures 3.4-3.6, a best fit of the experimental results gave a $\beta$ value of 16 for $10 \mu \mathrm{m}$ alumina particles, 15 for $25 \mu \mathrm{m}$ alumina particles (identical to a previously derived result [25], thus confirming that the method is reliable), and 14 for $50 \mu \mathrm{m}$ silica glass particles blasted at a pressure of $200 \mathrm{kPa}$ and using a $0.76 \mathrm{~mm}$ nozzle (Table 3.1) [26]. In Ref. [15], the relative degree of focus from various nozzles was explained in terms of interactions between the particles and the interior wall of the nozzle, which in turn depends on the nozzle wall roughness. $\beta$ is higher for more focused jets, and lower for divergent jets. For rougher nozzles, the relative degree of particle interaction with the nozzle wall is higher, resulting in a more divergent jet (lower $\beta$ ). In the present work, for the same particle type, the jet was found to be slightly more focused for the smaller particles (i.e., the $\beta$ value was higher for the $10 \mu \mathrm{m}$ alumina particles than for the $25 \mu \mathrm{m}$ alumina particles). This is likely because for the $10 \mu \mathrm{m}$ alumina particles, the particle velocity is higher (due to their lower mass) [4] and the $r_{n} / r_{p}$ ratio (see Section 3.4.2.1) is larger, than for $25 \mu \mathrm{m}$ alumina particles, all other parameters being equal. The higher particle velocity and $r_{n} / r_{p}$ ratio reduce the probability of particle interactions with the internal nozzle wall and decrease the overall effect of nozzle wall roughness on divergence (Section 3.4.2.1), resulting in a more focused jet $[4,15]$.

The jet was found to be slightly more focused for the $10 \mu \mathrm{m}$ and $25 \mu \mathrm{m}$ angular alumina particles than for the silica $50 \mu \mathrm{m}$ glass beads. There are two likely explanations for this. The first explanation is that the $50 \mu \mathrm{m}$ silica glass particles have lower velocities (due to their higher mass and lower drag coefficient [15]) and a lower $r_{n} / r_{p}$ ratio, than the $10 \mu \mathrm{m}$ and $25 \mu \mathrm{m}$ alumina particles. This increases the probability of particle interactions with the internal nozzle wall and increases the overall effect of nozzle wall roughness on divergence (Section 3.4.2.1), which results in a less focused jet $[4,15]$. A second explanation is that the angular particles, due to their irregular shape, have a higher tendency to rotate upon impact with the internal nozzle wall, and thus convert more of their incident energy to rotational energy than spherical particles. The angular particles return less of the incident energy normal to the nozzle wall in the form of rebound linear velocity normal to the wall, and thus experience shallower rebound angles upon 
colliding with the nozzle internal wall, than the spherical silica glass particles. Thus, on average, the exiting jet is less divergent [15] in the case of angular particles [26].

The results seem to imply that the effect of particle size on $\beta$ is more pronounced than the effect of particle type. Since a $150 \%$ increase in particle size (from 10 to $25 \mu \mathrm{m}$ ) for the alumina caused a small reduction in $\beta$ from 16 to 15 , it would be expected that for $50 \mu \mathrm{m}$ alumina particles (a further $100 \%$ increase), $\beta$ would be less than 15 (i.e., probably closer to 14 , which is the case for the $50 \mu \mathrm{m}$ silica glass beads) (Table 3.1). Thus, it can be said that the effect of particle type, and hence shape, (in this size range) has a minimal effect on the resulting divergence. However, in order to draw more direct comparisons, further work needs to be done for different particle types of the same size.

\subsubsection{Effect of Nozzle Size and Type}

\subsubsection{Effect of Nozzle Size (Round Nozzles)}

From Figures 3.7-3.10, a best fit of the experimental results at $200 \mathrm{kPa}$ using the $0.46 \mathrm{~mm}$ H.P. and $1.5 \mathrm{~mm}$ H.P nozzles, gave $\beta$ values of 12 and 20 , respectively, in the case of $25 \mu \mathrm{m}$ alumina particles, and $\beta$ values of 9 and 18, respectively, in the case of $50 \mu \mathrm{m}$ silica glass particles (Table 3.1). These results imply that the jet is more focused for larger nozzles and less focused for smaller nozzles, all other variables being the same. For a given particle size, the $r_{n} / r_{p}$ ratio (maximum amount of particles that can fit across a round nozzle cross section), where $r_{n}$ is the nozzle radius and $r_{p}$ is the particle radius, is higher for larger nozzles, meaning that there is a larger inter-particle spacing [26]. Thus, for larger nozzles, it is logical to expect a smaller percentage of particles that can fit in a nozzle cross section to collide with the interior nozzle walls, than for smaller nozzles. The overall effect of nozzle wall roughness on divergence is thus smaller for larger nozzles and as a result, the jet is more focused. Although the mass flow rate was higher for larger nozzles (Table 3.1), and thus more particles exited into the jet for the same amount of time, this had little effect on the divergence since the mass flow rates used in these experiments were not sufficiently high so as to further influence divergence (refer to Section 3.4.5).

It should be noted that these results agree with the predictions made in Section 3.4.1, since the jet is more focused for angular alumina particles than for silica glass particles, 
regardless of what size of nozzle is used. Finally, it is noteworthy that high performance (H.P.) nozzles mentioned above have a slightly more focused jet than conventional nozzles, presumably due to the slightly lower interior roughness claimed by the manufacturer. However, this difference is small and the results are still comparable.

For cases where the $0.46 \mathrm{~mm}$ H.P. nozzle was used (Figures 3.7 and 3.9), the piecewise Weibull distribution (equation (3.9)) modeled the experimental data, as opposed to the standard Weibull distribution (equation (3.5)). The new distribution introduces a 'hump'; i.e., more particles appear off of the jet centre than directly in the centre. The reason for this hump is not clear, but reasons for this might be due to a combination of amplified roughness effects and a significantly small inter-particle spacing in the nozzle cross section (i.e., $r_{n} / r_{p}<20$ ). Since the nozzle size is relatively small, the effect of nozzle roughness on the interaction between the nozzle walls and the particles in contact with it, is more pronounced. In Figures 3.6 and 3.13, where $50 \mu \mathrm{m}$ silica glass particles were blasted at pressures of 200 and $300 \mathrm{kPa}$ using a $0.76 \mathrm{~mm}$ nozzle, although $r_{n} / r_{p}<20$, this effect was not seen since a larger nozzle was used.

For round nozzles, this effect has not been directly reported in the literature. In any event, it is safe to say that for AJM applications, the use of such small nozzles along with an $r_{n} / r_{p}$ ratio of less than 20 should be viewed with caution since for these cases the jet behaves in an unpredictable manner and interference behaviour is difficult to generalize [12]. Since the interference behaviour is of prime interest in the present work, the use of such small nozzles is undesirable. In addition, since for these cases the jet was very divergent (i.e., $\beta=12$ in Figure 3.7 and $\beta=9$ in Figure 3.9) and the mass flow rate was low (i.e., flow rate $=0.36 \mathrm{~g} / \mathrm{min}$ in Figure 3.7 and flow rate $=0.23 \mathrm{~g} / \mathrm{min}$ in Figure 3.9 ), it would take a long time to machine a feature because the erosive energy at the surface is not only scattered, but is also quite low. Therefore, these nozzles should only be used for applications where a small circular profile of relatively shallow depth is desired.

\subsubsection{Effect of Nozzle Type (Rectangular Nozzles)}

From Figures 3.14 and 3.15 , a best fit of the experimental results gave $\beta$ values of 13 and 13.5 for $50 \mu \mathrm{m}$ silica glass particles blasted at $200 \mathrm{kPa}$ using $3.2 \times 0.2 \mathrm{~mm}$ and $3.8 \times 0.3 \mathrm{~mm}$ rectangular nozzles, respectively, regardless of scanning direction (Table 3.1). The results again 
show that the jet is more focused for larger nozzles for the same reasons as stated in Section 3.4.2.1. $M\left(X^{*}\right) / M(0)$ followed the piecewise Weibull distribution (equations (3.12), (3.13) and (3.14)). The new distribution introduces a 'hump' just left or right of the jet center. This effect might be caused by the significant influence of nozzle roughness resulting from the small nozzle size and a low $(w / 2) / r_{p}$ ratio for these cases (i.e., 4 and 6 for $w=0.2 \mathrm{~mm}$ and $w=0.3 \mathrm{~mm}$, respectively (Table 3.1), and is explained in the same way as for smaller round nozzles discussed in the previous section.

This effect has not been directly reported in the literature. However, by examining the work of Ref. [19] for modeled and measured particle density distributions across the nozzle cross section for comparatively small rectangular nozzles $(w=1.5 \mathrm{~mm})$, it was discovered that there was a higher particle count just left of right of the jet center than at the very center (see Figure 4 of Ref. [19]). However, these findings merit further study so that these claims can be verified.

$M\left(Y^{*}\right) / M(0)$ followed the standard Weibull distribution (equation (3.17)) because the jet is largely unconstrained in that direction (i.e., the $(l / 2) / r_{p}$ ratio is high (Table 3.1)). In addition, it is interesting to note that the overall jet divergence was the same (i.e., $\beta$ remains the same) for measurements across the $\mathrm{x}$ - and $\mathrm{y}$ - axes.

\subsubsection{Effect of Pressure}

From Figures 3.11-3.13, a best fit of the experimental results gave $\beta$ values of 20, 17, and 12 for $10 \mu \mathrm{m}$ alumina, $25 \mu \mathrm{m}$ alumina, and $50 \mu \mathrm{m}$ silica glass particles, respectively, blasted at $300 \mathrm{kPa}$ using a $0.76 \mathrm{~mm}$ nozzle (Table 3.1). The results for $10 \mu \mathrm{m}$ and $25 \mu \mathrm{m}$ alumina particles show that the jet is more focused at higher pressures, when compared to results using $200 \mathrm{kPa}$, for otherwise the same conditions, where $\beta$ values of 16 and 15 were obtained, respectively (Figures 3.4-3.5). This is likely because particle velocity increases with increasing pressure [16], reducing the probability of particle interactions with the internal nozzle wall, as explained in Section 3.4.1. However, for $50 \mu \mathrm{m}$ silica glass particles, the results show an opposite effect - the jet is more divergent for $300 \mathrm{kPa}(\beta=12)$, when compared to results using $200 \mathrm{kPa}(\beta=14)$, under otherwise identical conditions. This may result from the fact that the $r_{n} / r_{p}$ ratio is lower for the larger silica glass particles (i.e., below 20), when compared to the alumina particles $\left(r_{n} / r_{p}\right.$ $=76$ and $r_{n} / r_{p}=30.4$ for $10 \mu \mathrm{m}$ alumina and $25 \mu \mathrm{m}$ alumina particles, respectively). This causes 
a larger percentage of the total number of particles that can fit in a nozzle cross section to interact with the interior nozzle walls. Thus, for this case, higher velocity might increase particle interactions with the internal nozzle wall because the effect of particle spacing (i.e., low $r_{n} / r_{p}$ ratio) is more pronounced than the effect of pressure for the silica glass particles, and as a result the jet is more divergent (Table 3.1). The combination of these competing factors causes the jet to behave in this unexpected manner. It should be again noted that the results for a pressure of $300 \mathrm{kPa}$ agree with the predictions made in Section 3.4.1, since the jet is more focused for angular alumina particles than for silica glass particles, regardless of what size of nozzle is used, and is more focused for smaller particle sizes, as opposed to larger ones.

\subsubsection{Effect of Standoff Distance and Exposure Time}

$M\left(R^{*}\right) / M(0)$ in Figures 3.6, 3.9, and 3.13 were measured at two different standoffs. Since the data for all the cases was normalized and non-dimensionalized with respect to the standoff (i.e., plotted against $r^{*}=r / d$ ), the results show that the distribution was independent of standoff distance, so that the particles followed straight trajectories from nozzle to catcher [15]. This can be seen in the above figures since the experimental data for two different standoffs for each case fall on the same curve (i.e., equation (3.5) in Figures 3.6 and 3.13 and equation (3.9) in Figure 3.9). It is thus reasonable to assume that all the results are independent of the standoff distance (Figures 3.4-3.15).

It should be noted that for $d<200 r_{n}$ [28] (as in most cases here, see Table 3.1), the air jet velocity can still have an influence on the way particles rebound from a flat target. Since the targets used here were 'catching' cylinders and not a flat target, these effects are expected to be minimal in most cases. For example, in the case of the $0.46 \mathrm{~mm}$ catcher having a wall thickness of only $100 \mu \mathrm{m}$, (Figures 3.5-3.10, 3.12-3.15), there were likely very few rebounding particles. However, when the $1.2 \mathrm{~mm}$ catcher with a wall thickness of $760 \mu \mathrm{m}$ was used (Figures 3.4 and 3.11), particles aimed towards the collection cylinder face could rebound from the collection cylinder face and interfere with incoming particles, resulting in the presence of the small 'hump' (i.e., the off center maximum) present in Figures 3.4 and 3.11 (see Section 3.4.7). Since the influence of the air jet on interference effects depends on the standoff distance, a standoff distance dependent effect might have been introduced in these cases. 
Finally, it should be noted that the results, as expected, were independent of the exposure time, because all exposure times were far greater than the time required to reach steady state.

\subsubsection{Effect of Particle Mass Flow Rate}

The particle mass flow rate was found to vary with pressure, particle type and size, and nozzle type and size (Figures 3.4-3.15). The mass flow rate was found to increase with increasing $r_{n} / r_{p}$ ratio and increasing pressure (Table 3.1). Since the jet is less constrained by the internal nozzle walls for larger nozzles or smaller particles, more particles are permitted to exit the nozzle into the jet, for the same exposure time. Hence, when compared to larger ones of the same material, smaller particles exited the nozzle at higher mass fluxes, despite having lower masses. Particles also travel faster at higher pressures [16] which results in more particles exiting the nozzle into the jet, for the same exposure time. In addition, for the same $r_{n} / r_{p}$ and pressure, the angular alumina particles had a higher mass flux than the silica glass beads (compare Figure 3.5 and Figure 3.10 in Table 3.1 for $r_{n} / r_{p}=30$ and pressure of $200 \mathrm{kPa}$ ). This is due to the higher mass, and hence lower velocity, of the silica glass beads when compared to alumina particles.

Figure 3.6 shows $M\left(R^{*}\right) / M(0)$ for the $50 \mu \mathrm{m}$ silica glass particles for two different particle mass flow rates. An increase in the particle mass flow rate decreases the relative distance between particles, in the nozzle radial plane, as well as the nozzle axial plane $[1,4,10]$. However, since the mass flow rates used in these experiments were not sufficiently high so as to further affect particle interaction with the internal nozzle wall (in addition to particle velocity and nozzle roughness), there was little effect on the jet divergence (i.e., the plume shape) and thus the results should not be significantly affected by the mass flow rate (compare the two cases in Figure 3.6).

It should be noted that $M\left(R^{*}\right) / M(0)$ can depend on the mass flow rate for low $r$ for the cases in Figures 3.4 and 3.11, where the flow rate was high and a $1.2 \mathrm{~mm}$ catcher with a minimum wall thickness of $780 \mu \mathrm{m}$ was used, because of the possibility of particle interference between incoming particles and those rebounding from the collection cylinder face. However, this should not affect the measurement of $\beta$ (see Section 3.4.7). 


\subsubsection{Effect of Particle Collection Cylinder Size}

$M\left(R^{*}\right) / M(0)$ in Figure 3.5 was measured using a $0.46 \mathrm{~mm}$ collection cylinder. The same value of $\beta$ was obtained in Ref. [25] using a $1.2 \mathrm{~mm}$ collection cylinder (i.e., $\beta=15$ ) for the same case, confirming $M\left(R^{*}\right) / M(0)$ is independent of $r_{c}$. However, $r_{c}$ should be large enough so as to allow sufficient amount of mass to be collected for a specified exposure time, and small enough so as to obtain a sufficient amount of experimental data points across the jet.

\subsubsection{Advantages over Scar radius Method and Sources of Error}

The present method of extracting $\beta$ has a number of advantages over the erosion scar radius method used in [15-17], where $\beta$ was obtained from blasting particles on flat aluminum targets covered with a thin dye (Section 1.1.4). The present method is likely more accurate because it eliminates concerns regarding irregularities in dye thickness, and uncertainty in scar radius measurement, both of which can strongly affect the results. Moreover, the present method is more direct, which can also be used in high flux measurements, something that is not possible for the scar radius method, due to major concerns with collisions between incoming particles, and those rebounding from the flat plate. In addition, for measurements at low fluxes, the duration of the experiments can be much less than the case of scar radius measurements. For the present method, even at low fluxes, it would only take several minutes to collect a measurable amount of mass. However, it might take several hours to erode the scar to various degrees at a low particle flux [15-17].

However, there are a number of possible sources of error in the present method. Nozzle misalignments seem to have a minimum effect on the results but can cause variability in the experimental data (Figures 3.4-3.15; Tables A-1a-A-18a). Wear of the collection cylinder face is only a concern when using alumina particles. However, during the whole course of the experimental run, the collection cylinder face experienced negligible wear (refer to Section 3.2.1). In addition, for most of these cases, the exposure time was kept to a minimum and the standoff distance to a maximum to minimize this effect even further.

The major source of error that may be a cause for concern is the possibility of particle interference between incoming particles and those rebounding from the collection cylinder face. 
This effect can be seen in Figures 3.4 and 3.11, where the flow rate was high and a $1.2 \mathrm{~mm}$ catcher with a minimum wall thickness of $780 \mu \mathrm{m}$ was used, where $M\left(R^{*}\right) / M(0)$ was higher just left or right of the catcher than at the jet center. When the collection cylinder is positioned at the jet center (i.e., coincident and parallel to the nozzle axis (refer to top view of Figure 3.3)), the proportion of the jet being collected is more dense and particles aimed towards the cylinder travel in straighter trajectories. When some of the particles collide with the cylinder face, the interference is negligible since they rebound at angular trajectories that do not interfere with particles aimed towards the cylinder opening. However, when the cylinder is positioned just to the left or right of nozzle axis, the particles travel at oblique angles and thus some particles that rebound from the cylinder edge positioned closer to the nozzle axis collide with particles that are aimed towards the cylinder opening. The rebounding particles are thus diverted towards the cylinder opening while the arriving particles are diverted away from the cylinder opening. Since the rebounding particles that are now collected, instead of the original arriving particles, are in a portion of the jet having higher stream density than the original arriving particles, the results show a slightly higher $M\left(R^{*}\right) / M(0)$ just left or right of the jet center than at the very center (i.e., a small 'hump' is present). The influence of the air jet [28] can also affect the way the particles interfere for these cases and thus can contribute to the presence of this small 'hump' near the jet center in Figures 3.4 and 3.11 (refer to Section 3.4.4). Although this occurred for these two cases, the rest of the cases seemed to show no interference effects, where the larger 'humps' visible in Figures 3.7, 3.9, 3.14 and 3.15 were more likely caused by a combination of amplified roughness effects and a significantly small inter-particle spacing in the nozzle cross section. For higher $r$, the jet is less dense and interference is less pronounced so the experimental results followed the Weibull distribution. Hence, measurements at the center and at higher $r$ (i.e., the last 4 measurements) matched the Weibull distribution more accurately. 


\section{Chapter 4 Measurement of the Particle Velocity Distribution in Micro- abrasive Jets}

\subsection{Motivation}

As mentioned in Chapter 3, in order to control and predict the resulting erosion patterns on the target surface, and thus the interference patterns, the particle jet must be accurately characterized by obtaining not only the spatial particle distribution, but also the velocity distribution across the jet $[15,17,25]$. This chapter describes a novel technique for obtaining the velocity distribution, using the results of particle catcher experiments (Chapter 3) along with eroded profiles obtained from shallow depth micromachining experiments and velocity exponents obtained from erosion rate experiments, at various jet conditions. As was mentioned in Chapter 1, for relatively shallow profiles, the profile can be related to the product of the particle velocity raised to a velocity exponent and the particle mass flux [20]. This would enable one to extract the velocity distribution if the flux distribution is known.

The velocity distribution is needed as an input for an analytical model that can realistically predict particle interference and surface erosion rates for a diverse set of conditions (Chapter 5). 


\subsection{Measurement of the Particle Velocity Distribution across the Jet \\ 4.2.1 Erosion Rate Experiments to Obtain the Velocity Exponent: Experimental Setup and Procedure}

The velocity exponent, $k$, which depends on the particle velocity, material and geometry, and the nature of the substrate [20], can be obtained experimentally, as was done, for example in Refs. [25,29]. In Ref. [25], a $k$ of 1.43 was obtained by first measuring erosion rates on borosilicate glass at various incident angles for $25 \mu \mathrm{m}$ angular aluminum oxide blasted at a pressure of $200 \mathrm{kPa}$ and a flow rate of $2.83 \mathrm{~g} / \mathrm{min}$ using a $0.76 \mathrm{~mm}$ round nozzle at a constant standoff distance of $20 \mathrm{~mm}$. This approach was followed here for the following cases: $10 \mu \mathrm{m}$ angular aluminum oxide blasted at pressures of 200 and $300 \mathrm{kPa}$ and for $25 \mu \mathrm{m}$ angular aluminum oxide blasted at a pressure of $300 \mathrm{kPa}$, all using a $0.76 \mathrm{~mm}$ round nozzle at a constant standoff distance of $30 \mathrm{~mm}$ (measured from the nozzle to the glass surface). The erosion rate was obtained by dividing the average mass loss of the substrate (g) during the exposure time of 1 minute by the flow rate $(\mathrm{g} / \mathrm{min})$ for that specific case (see Appendix B, Tables B- $1 \mathrm{~b}, \mathrm{~B}-8 \mathrm{~b}$, and B-9b). The measurement of mass loss of the substrate per minute for each case was repeated once and found to be repeatable, with standard deviations for each experiment, for the three cases, varying between $0.46-19.2 \%$, and for majority of cases between $0.46-7.4 \%$ (Tables B-1b, B-8b, and B-9b).

\subsubsection{Shallow Profile Experiments: Experimental Setup and Procedure}

Profiles were eroded by blasting $10 \mu \mathrm{m}$ and $25 \mu \mathrm{m}$ angular alumina and $50 \mu \mathrm{m}$ spherical silica glass particles on borosilicate glass plates (Borofloat, Schott North America Inc., Elmsford, NY, USA) of $5 \mathrm{~mm}$ thickness using the microblaster and shutter setup (Comco Inc., Burbank, CA, USA) (see Section 3.2.1). Experiments were all performed at steady state at standoff distances of 10-30 mm at normal incidence (i.e., $\alpha=90^{\circ}$ ) at mass flow rates of $0.23-4.75 \mathrm{~g} / \mathrm{min}$ under constant blasting pressures of 200 and $300 \mathrm{kPa}$ using round $0.46 \mathrm{~mm}$ H.P., $0.76 \mathrm{~mm}$ and $1.5 \mathrm{~mm}$ H.P. nozzles (Comco Inc., Burbank, CA, USA). These conditions matched those of Chapter 3 since the profiles will be related to the results of the particle catcher experiments.

Only relatively shallow profile depths, $\delta$, (maximum depth $\approx 150 \mu \mathrm{m}$ ) were considered for reasons which will be explained shortly. The erosion times varied between 2-300 s, and were chosen as a compromise between obtaining a smooth, measurable profile and maintaining a 
relatively shallow profile depth. The erosion times for profiles machined using the spherical silica glass particles, as opposed to using angular alumina, were considerably longer for a number of possible reasons. For the same jet conditions, the glass beads have lower velocities and thus they carry less erosive energy to the surface than the alumina particles. In addition, because of their spherical shape, it takes longer for them to erode the glass surface because they are not as sharp as the angular alumina particles. (refer to Appendix B, Tables B-1-B-11). For each case, two profiles were machined in order to assess repeatability.

The blasting profiles were analyzed using a stylus tip profilometer (Form Talysurf Intra, Taylor Hobson Ltd., Leicester, England) with a lateral and depth resolution of $0.2 \mu \mathrm{m}$ and $3 \mathrm{~nm}$, respectively. All experiments were filtered using a Gaussian high pass filter with a cut-off length of $0.25 \mathrm{~mm}$ using a waviness filter mode. Since the profiles were machined at normal incidence, they were axis-symmetric, and as a result only the 2-dimensional (2D) profile needed to be measured. The assessment lengths for the profiles were between $4.25-9.75 \mathrm{~mm}$, over which $8,500-19,500$ data points were generated using a scanning speed of $1 \mathrm{~mm} / \mathrm{s}$. For each profile, two passes through the profile centerline were made, with the deepest pass being taken as representing the actual profile cross section. An example of a blasting profile is depicted in Figure 4.1, where $\delta(r)$ represents the profile depth as a function of $r$, measured from the jet center. Measurements for the blasting profiles for several cases corresponding to particle spatial distributions of Section 3.3 are recorded in Appendix B, Tables B-1-B-11.

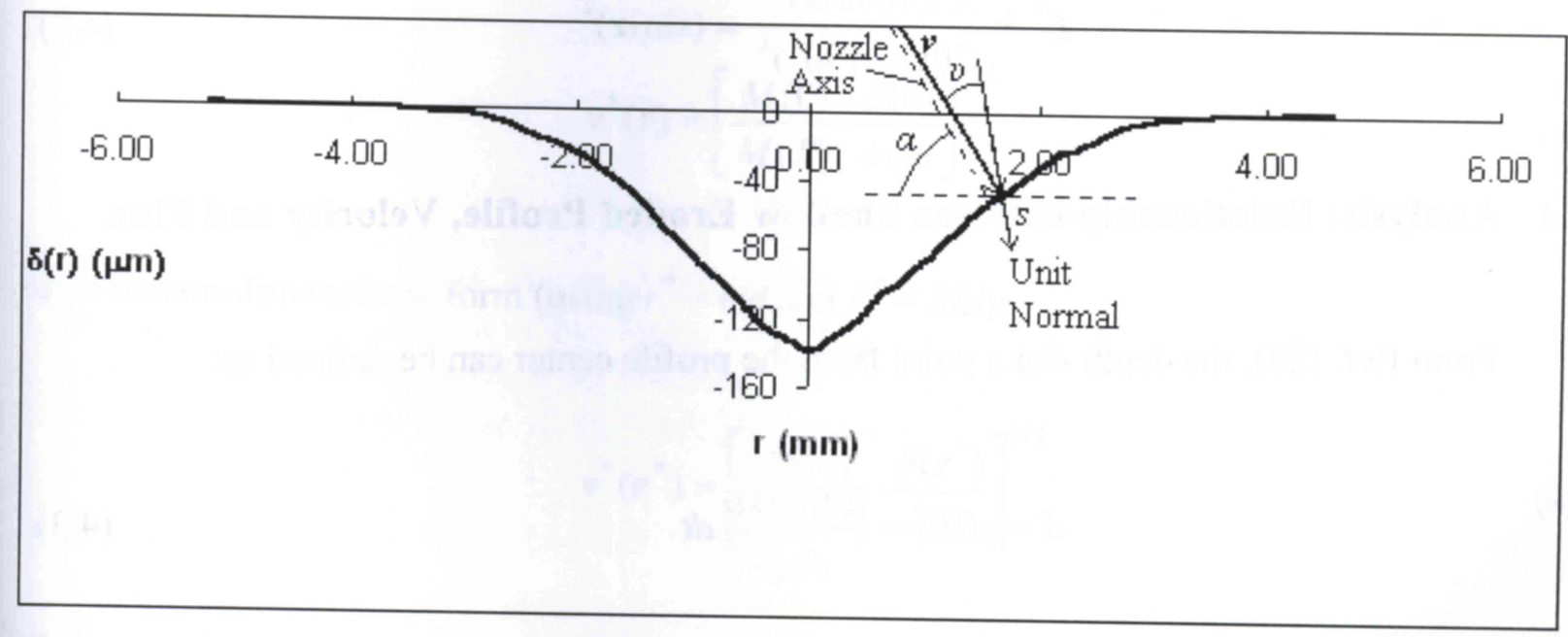

Figure 4.1: Example of a shallow profile (profile depth, $\delta(r)$, as a function of $r$ ) (corresponding to Table B-2) for $25 \mu \mathrm{m}$ angular aluminum oxide blasted at $d=30 \mathrm{~mm}$ for $10 \mathrm{~s}$ at a pressure of $200 \mathrm{kPa}$ and a flow rate of $2.83 \mathrm{~g} / \mathrm{min}$ using a $0.76 \mathrm{~mm}$ round nozzle. 


\subsubsection{Analysis: Relationship between Erosion Rate and Velocity Exponent}

As was mentioned in Chapter 1, the erosion rate depends on the magnitude of the particle impact velocity, $|\boldsymbol{v}|$ [22]. For oblique incidence, the erosion rate is approximately proportional to the normal component of the particle impact velocity at the surface [20, 21, 23]. The erosion rate, $E$ (i.e., the mass of target material removed per mass of particle incident on the target), can be defined as [20,21]:

$$
E=C(|\mathbf{v}| \cos v)^{k}
$$

where $C$ is an empirical constant, which is a weak function of $v, v$ is the angle between the incident velocity vector $\boldsymbol{v}$ and the unit normal on the profile surface (Figure 4.1), and $k$ is the velocity exponent.

The erosion rate $E$ can be normalized by dividing $E$ by the value for normal impact (at $\alpha$ $=90^{\circ}$ ). Using equation (4.1) and the fact that $\alpha+v=\pi / 2$ for shallow profiles (when the unit normal is almost perpendicular to the surface in Figure 4.1), the normalized erosion rate, $E^{*}$, can be defined as:

$$
E^{*}=\frac{C(|\mathbf{v}| \sin \alpha)^{k}}{C\left(|\mathbf{v}| \sin 90^{\circ}\right)^{k}}=(\sin \alpha)^{k}
$$

\subsubsection{Analysis: Relationship between Shallow Eroded Profile, Velocity and Flux}

From Ref. [20], the depth $\delta$ at a point from the profile center can be defined as:

$$
\delta=s(d t)=\frac{E \Phi \cos v}{\rho_{\text {sub }}} d t
$$

where $s$ is the velocity at the surface in the direction of the unit normal on the surface (Figure $4.1), d t$ is the infinitesimal time it takes for the surface to reach a depth $\delta, \Phi$ is the particle mass 
flux (mass of particles arriving to the target area per second), and $\rho_{\text {sub }}$ is the density of the substrate. Since the profiles were machined at normal incidence, and since only relatively shallow surface depths were considered, $v \rightarrow 0$. Using equations (4.1) and (4.3), the normalized depth distribution (with respect to the jet center), $\delta(r) / \delta(0)$, can be expressed as:

$$
\frac{\delta(r)}{\delta(0)}=\left.\frac{\rho_{\text {sub }} C(|\mathbf{v}| \cos v)^{k} \Phi(r) \cos (v) d t}{\left.\rho_{\text {sub }} C(|\mathbf{v}| \cos v)^{k} \Phi(r) \cos (v) d t\right|_{r=0}}\right|_{v \rightarrow 0}=\frac{(|\mathbf{v}| \cos 0)^{k} \Phi(r) \cos (0)}{\left.(|\mathbf{v}| \cos 0)^{k} \Phi(r) \cos (0)\right|_{r=0}}=\left(\frac{v(r)}{v(0)}\right)^{k} \frac{\Phi(r)}{\Phi(0)}(4
$$

where $v(r)$ is the normal component (to the surface) of the incident velocity vector $v$, as a function of $r$. Since the collection times in the experiments of Section 3.3.1 were the same at $r=$ $R$ and $r=0, M(R) / M(0) \approx \Phi(R) / \Phi(0)$ at a particular location of the particle collection cylinder, $r$ $=R$, and equation (4.4) becomes:

$$
\frac{\delta(r)}{\delta(0)}=\left(\frac{v(r)}{v(0)}\right)^{k} \cdot \frac{M(r=R)}{M(0)}=v^{*}(r)^{k} \cdot \frac{M(R)}{M(0)}
$$

where $v^{*}(r)=v(r) / v(0)$, the normalized velocity distribution (with respect to the jet center). Solving for $v^{*}(r)$ yields:

$$
v^{*}(r)=\left[\frac{M(0)}{M(R)} \cdot \frac{\delta(r)}{\delta(0)}\right]^{1 / k}
$$

or in its non-dimensional form (using $r^{*}=r / d$ and $R^{*}=R / d$ ):

$$
v^{*}\left(r^{*}\right)=\left[\frac{M(0)}{M\left(R^{*}\right)} \cdot \frac{\delta\left(r^{*}\right)}{\delta(0)}\right]^{1 / k}
$$

where $v^{*}\left(r^{*}\right)$ is the normalized velocity distribution as a function of the non-dimensionalized radial coordinate $r^{*}$. It should be noted that $\Phi\left(R^{*}\right) / \Phi(0)$ is the normalized flux averaged over a 
unit ring area at a particular $r^{*}$ (see Figure 3.3 and equations (3.8)). For higher $r^{*}$ the unit ring area increases and thus $\Phi\left(R^{*}\right) / \Phi(0)$ decreases. However, $M\left(R^{*}\right) / M(0)$ is the normalized flux averaged over the opening of the particle catcher at a particular $r^{*}$ (see Figure 3.3 and equation (3.5)). Nonetheless, for higher $r^{*}, G(\theta) d \theta$ in equation (3.5) decreases and thus $M\left(R^{*}\right) / M(0)$ decreases in a similar manner as $\Phi\left(R^{*}\right) / \Phi(0)$ decreases with increasing unit ring area. Hence, $\Phi\left(R^{*}\right) / \Phi(0)$ can be closely approximated by $M\left(R^{*}\right) / M(0) . M\left(R^{*}\right) / M(0)$ values have the advantage of being raw data, and thus they are more accurate. In addition, for cases where the piecewise Weibull distribution was found (Figures 3.7 and 3.9), $\Phi\left(R^{*}\right) / \Phi(0)$ would have to be evaluated by using two probability distribution functions (i.e., equations (3.6) and (3.10)) for the two different $r^{*}$ ranges specified in equation (3.9). On the other hand, since $M\left(R^{*}\right) / M(0)$ values were already available, no evaluation was necessary. 


\subsection{Results}

\subsubsection{Velocity Exponent}

Figures 4.2-4.4 show that the best power regression fits of average $E^{*}$ vs. $\sin (\alpha)$ (i.e., equation (4.2) of Section 4.2.3) using the least squares method. Velocity exponents of $k=1.57$ and $k=1.44$ for $10 \mu \mathrm{m}$ angular aluminum oxide particles blasted at 200 and $300 \mathrm{kPa}$, respectively, and $k=1.59$ for $25 \mu \mathrm{m}$ angular aluminum oxide particles blasted at a pressure of $300 \mathrm{kPa}$, were obtained. The power law fits matched the experimental data very well, with $\mathrm{R}^{2}$ values varying between $0.97-0.99$. It should be noted that since the experimentally obtained $k$ is a fundamental erosion parameter, it should not vary with nozzle size, flow rate, and standoff (in the absence of interference effects), and thus the same $k$ was used for other nozzle sizes, flow rates and standoffs.

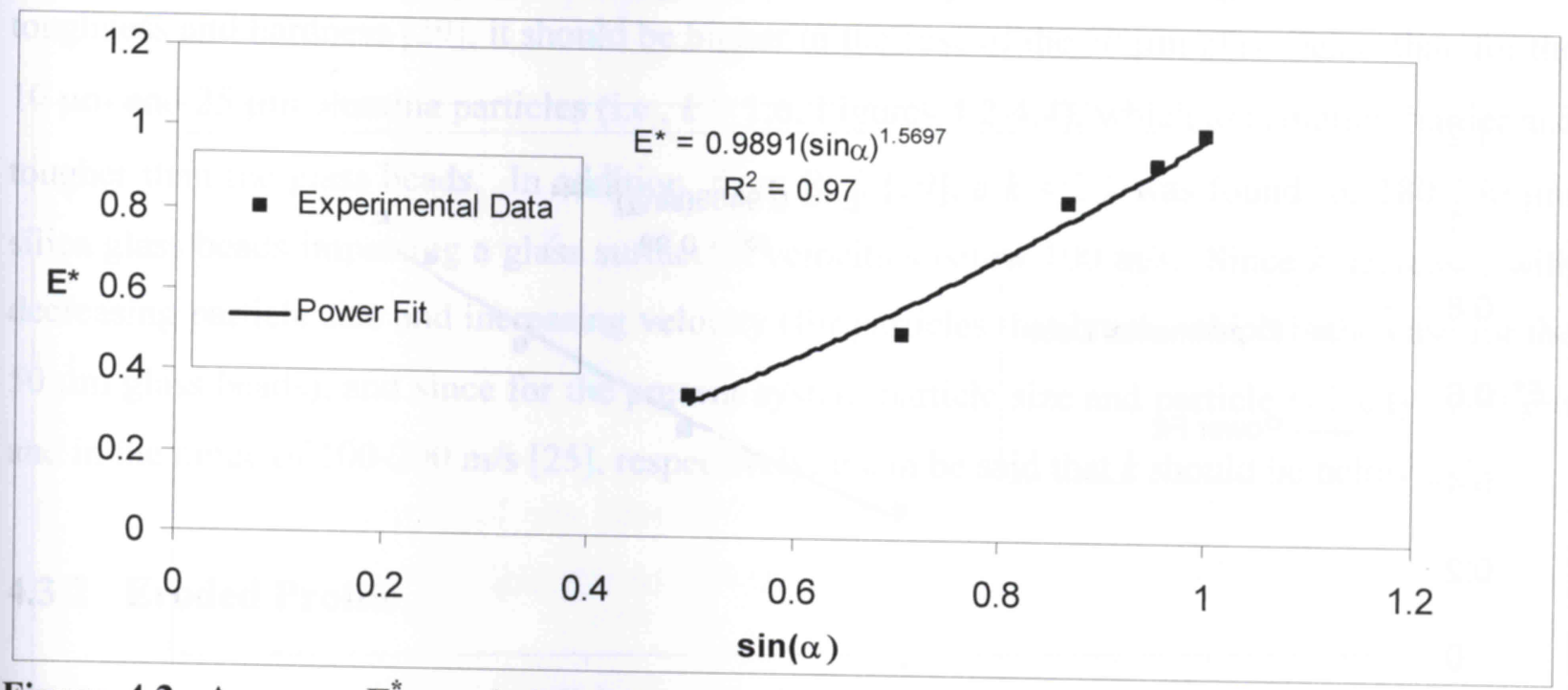

Figure 4.2: Average $E^{*}$ vs. $\sin (\alpha)$ (corresponding to data in Table B-1b). Best fit velocity exponent $k=1.57$. Erosion of Borofloat glass by $10 \mu \mathrm{m}$ aluminum oxide particles blasted at $d=30 \mathrm{~mm}$ for $60 \mathrm{~s}$ at pressure of $200 \mathrm{kPa}$ and flow rate of $3.97 \mathrm{~g} / \mathrm{min}$ using $0.76 \mathrm{~mm}$ round nozzle. - : Power regression fit to experimental data $\left(\square: R^{2}=0.97\right)$. 


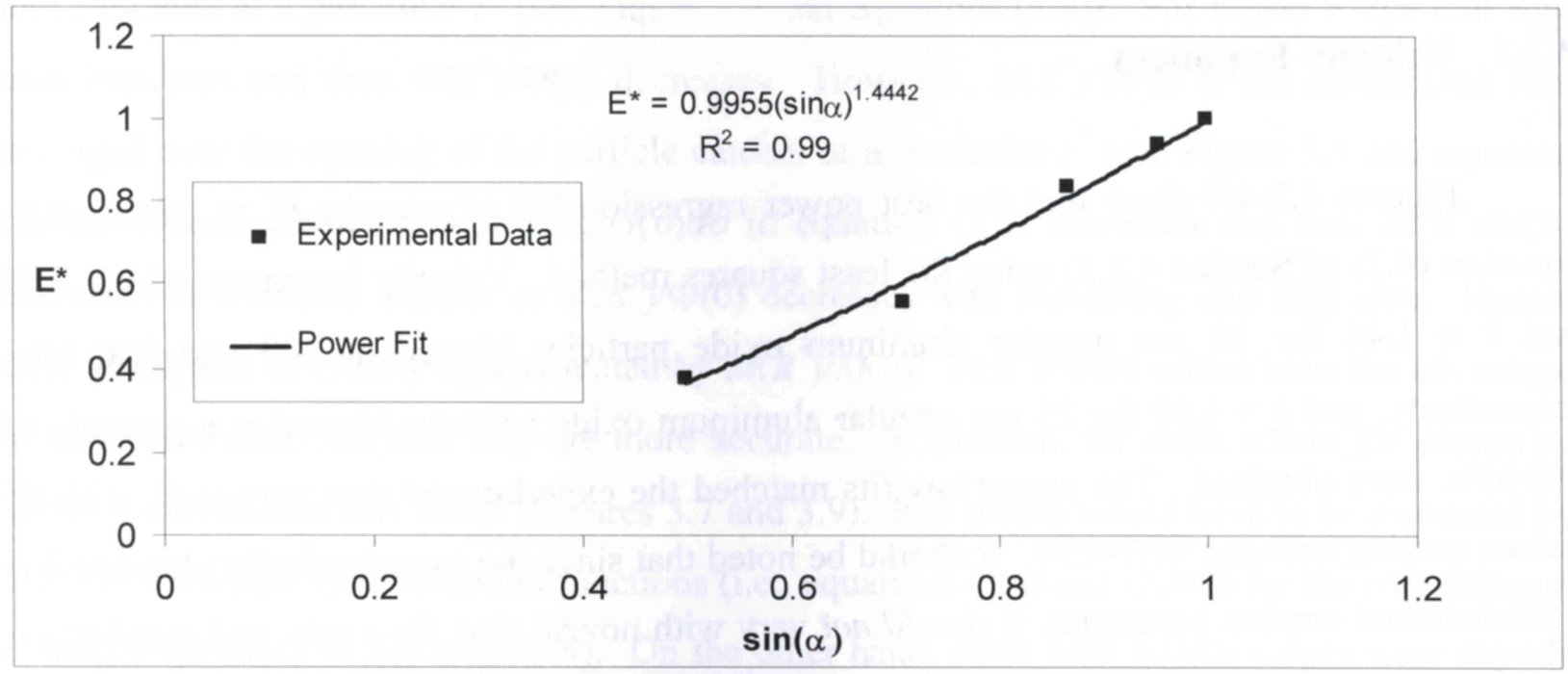

Figure 4.3: Average $E^{*}$ vs. $\sin (\alpha)$ (corresponding to data in Table B-8b). Best fit velocity exponent $k=1.44$. Erosion of Borofloat glass by $10 \mu \mathrm{m}$ aluminum oxide particles blasted at $d=30 \mathrm{~mm}$ for $60 \mathrm{~s}$ at pressure of $300 \mathrm{kPa}$ and flow rate of $4.20 \mathrm{~g} / \mathrm{min}$ using $0.76 \mathrm{~mm}$ round nozzle. - : Power regression fit to experimental data $\left(\square: R^{2}=0.99\right)$.

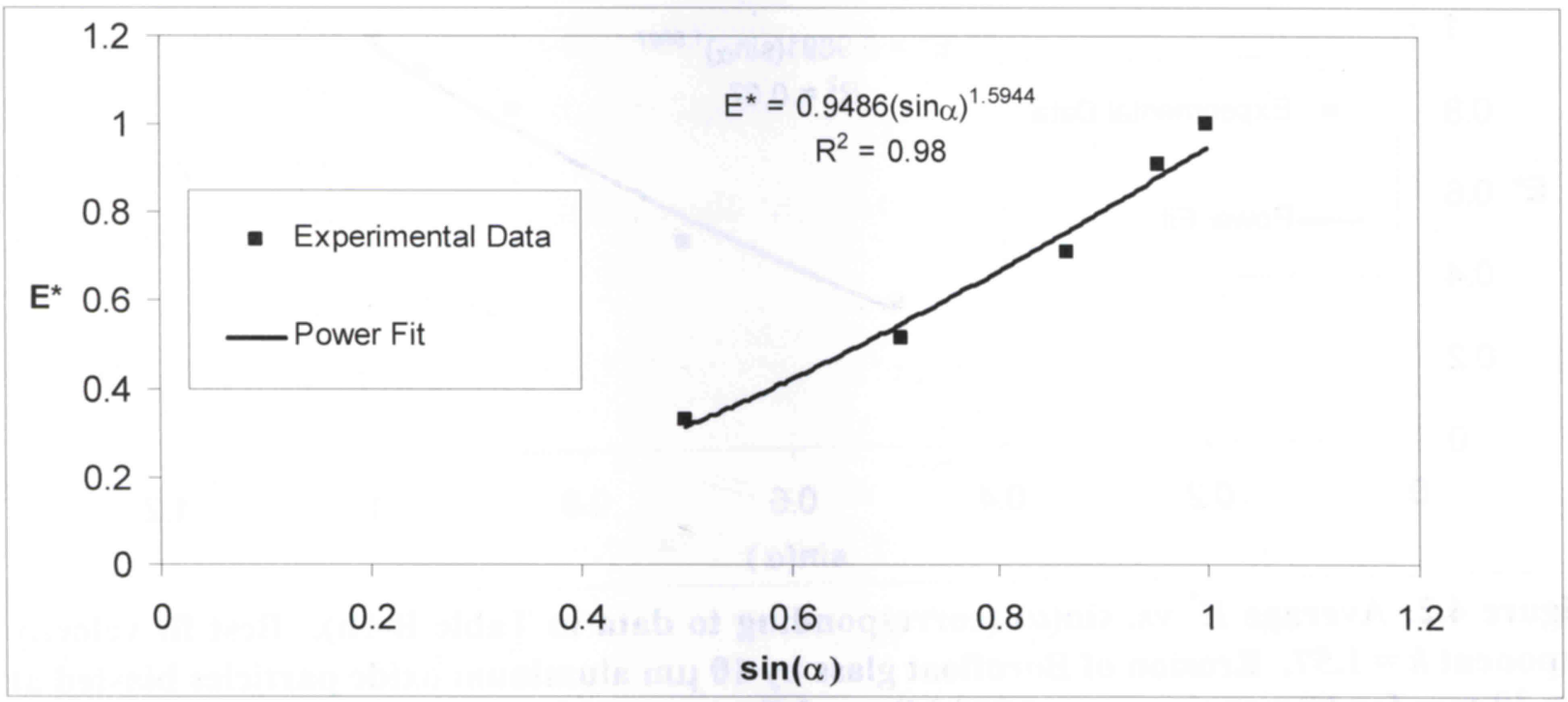

Figure 4.4: Average $E^{*}$ vs. $\sin (\alpha)$ (corresponding to data in Table B-9b). Best fit velocity exponent $k=1.59$. Erosion of Borofloat glass by $25 \mu \mathrm{m}$ aluminum oxide particles blasted at $d=30 \mathrm{~mm}$ for $60 \mathrm{~s}$ at pressure of $300 \mathrm{kPa}$ and flow rate of $3.10 \mathrm{~g} / \mathrm{min}$ using $0.76 \mathrm{~mm}$ round nozzle. - : Power regression fit to experimental data $\left(\square: R^{2}=0.98\right)$.

Normally, the velocity exponent is in the range of 2-4 for glass as the substrate with aluminum oxide as erodents [20,29]. However, in this work, the exceptionally small size of the erodents used caused $k$ to fall below this range. For the same substrate, the velocity exponent 
usually increases with larger particles size, lower toughness and hardness of erodents, and with higher particle velocity $[20,29]$. However, for particles that break more easily, (i.e., smaller particles), a higher velocity results in a lower $k$ because more of the kinetic energy of the particles is dissipated via particle breakage [30] and not towards erosion. Hence, for an increase in pressure (i.e., velocity), for the $10 \mu \mathrm{m}$ angular aluminum oxide, $k$ decreased from 1.57 to 1.44 (Figures 4.2 and 4.3) and for the $25 \mu \mathrm{m}$ angular aluminum oxide, $k$ increases from 1.43 [25] to 1.59 (Figure 4.4). In any event, this small differential in $k$ for the 10 and $25 \mu \mathrm{m}$ alumina does not significantly alter velocity distribution calculations (equation (4.7)).

The $k$ for the $50 \mu \mathrm{m}$ silica glass beads could not be experimentally obtained (an insufficient, and thus indeterminate, amount of mass was removed from the glass surface for a long erosion time). However, from the present analysis and from Ref. [29] it can be safely said that the $k$ for the $50 \mu \mathrm{m}$ silica glass beads impacting a glass target for the present system should be in the range of 1.6-2.7. Since $k$ increases with increasing size and a decrease in erodent toughness and hardness [29], it should be higher in the case of the $50 \mu \mathrm{m}$ glass beads than for the $10 \mu \mathrm{m}$ and $25 \mu \mathrm{m}$ alumina particles (i.e., $k>1.6$, Figures 4.2-4.4), which are smaller, harder and tougher than the glass beads. In addition, from Ref. [29], a $k \approx 2.7$ was found for 180-250 $\mu \mathrm{m}$ silica glass beads impacting a glass surface at velocities below $100 \mathrm{~m} / \mathrm{s}$. Since $k$ decreases with decreasing particle size and increasing velocity (for particles that break, which is the case for the $50 \mu \mathrm{m}$ glass beads), and since for the present system particle size and particle velocity is $50 \mu \mathrm{m}$ and in the range of $100-200 \mathrm{~m} / \mathrm{s}$ [25], respectively, it can be said that $k$ should be below 2.7 .

\subsubsection{Eroded Profile}

Normalized non-dimensionalized depth distributions (with respect to the jet center) as a function of $r^{*}, \delta\left(r^{*}\right) / \delta(0)$, for the two profiles for each experiment in Section 4.2.2, for several cases corresponding to particle spatial distributions of Section 3.3, are recorded in Appendix B, Tables B-1-B-11, where $\delta\left(r^{*}\right)$ represents the profile depth as a function of the nondimensionalized radial coordinate, $r^{*}=r / d$, measured from the jet center. The data was found to be very repeatable, with standard deviations for the normalized depth distributions, $\delta\left(r^{*}\right) / \delta(0)$, for the two profiles for each experiment, for all the experiments, varying between $0-4.68 \%$ (Tables 
B-1-B-11). It should be noted that the depth distributions for rectangular nozzles using $50 \mu \mathrm{m}$ spherical silica glass particles could not be obtained due to the formation of sharp craters, thus yielding uneven and unsymmetrical profiles.

\subsubsection{Resulting Velocity Distribution}

Equation (4.7) was used to calculate data points of $v^{*}\left(r^{*}\right)$ using average $M\left(R^{*}\right) / M(0)$ and $\delta\left(r^{*}\right) / \delta(0)$ values (Tables B-1-B-11). In these calculations, only the $\delta\left(r^{*}\right) / \delta(0)$ values at radial locations matching those at which the particle distributions of Chapter 3 (Appendix A) were also measured were used (i.e., from each profile, only depths corresponding to the same $r^{*} \mathrm{~s}$ as for $M\left(R^{*}\right) / M(0)$ were chosen). The velocity exponents, $k$, were obtained from Ref. [25] and Figures 4.2-4.4 for angular alumina, and lower $(k=1.6)$ and upper $(k=2.7)$ bounds of $k$ for silica glass beads. These data points were then plotted and fitted using a polynomial or linear regression fit for $v^{*}\left(r^{*}\right)$ using the least squares method (Figures 4.5-4.15):

$$
\begin{gathered}
v^{*}\left(r^{*}\right)=H_{p 1} r^{* 2}+H_{p 2} r^{*}+1 \\
v^{*}\left(r^{*}\right)=H_{l} r^{*}+1
\end{gathered}
$$

where $H_{p 1}, H_{p 2}$, and $H_{l}$ are the constants for the polynomial and linear regression fits. The $\mathrm{R}^{2}$ values for the regression fits were between $0.75-0.99$, for the majority of cases being between 0.90-0.99, indicating a fairly good fit. 


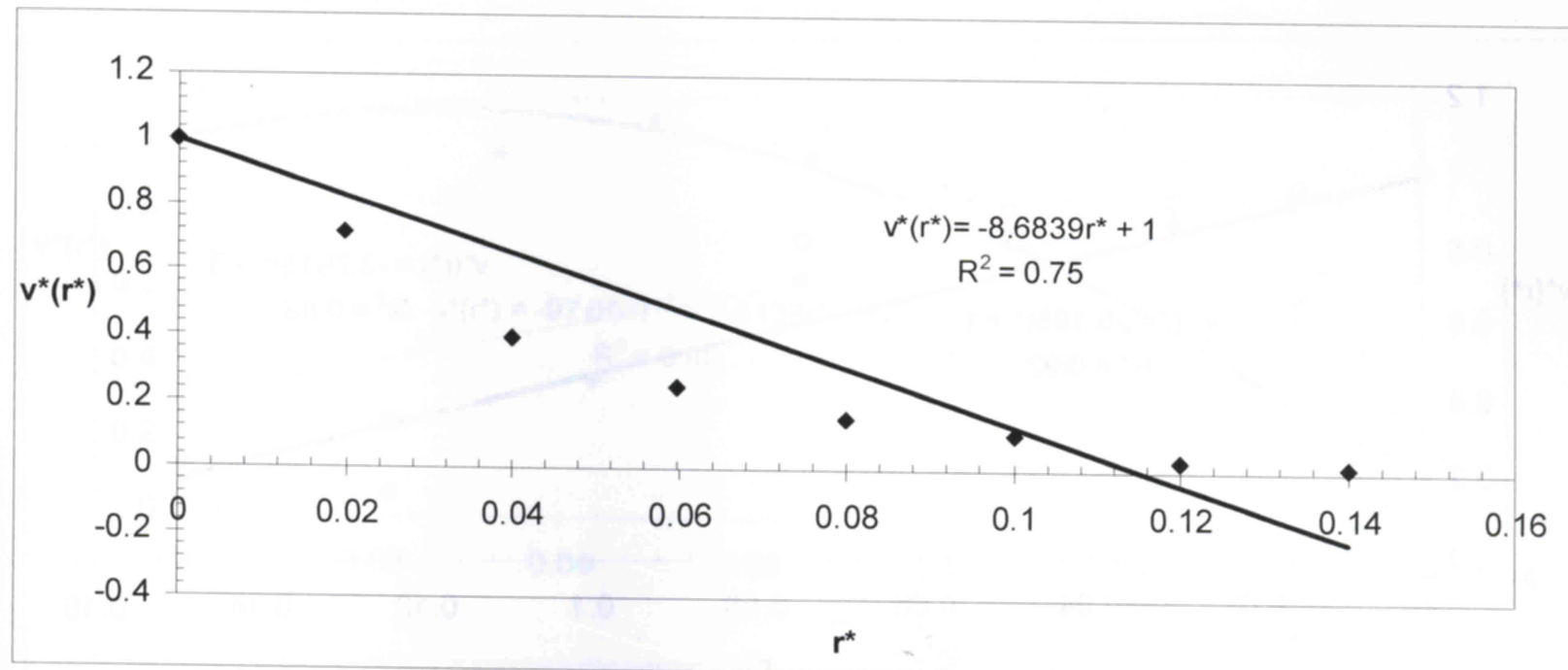

Figure 4.5: Calculated $v^{*}\left(r^{*}\right)$ vs. $r^{*}$ (corresponding to data in Table B-1a and particle spatial distribution in Figure 3.4) using $k=1.57$ (Figure 4.2) for $10 \mu \mathrm{m}$ angular aluminum oxide blasted at $d=30 \mathrm{~mm}$ at pressure of $200 \mathrm{kPa}$ and flow rate of $3.97 \mathrm{~g} / \mathrm{min}$ using $0.76 \mathrm{~mm}$ round nozzle. - - Linear regression fit to calculated data $\left(\bullet: R^{2}=0.75\right)$.

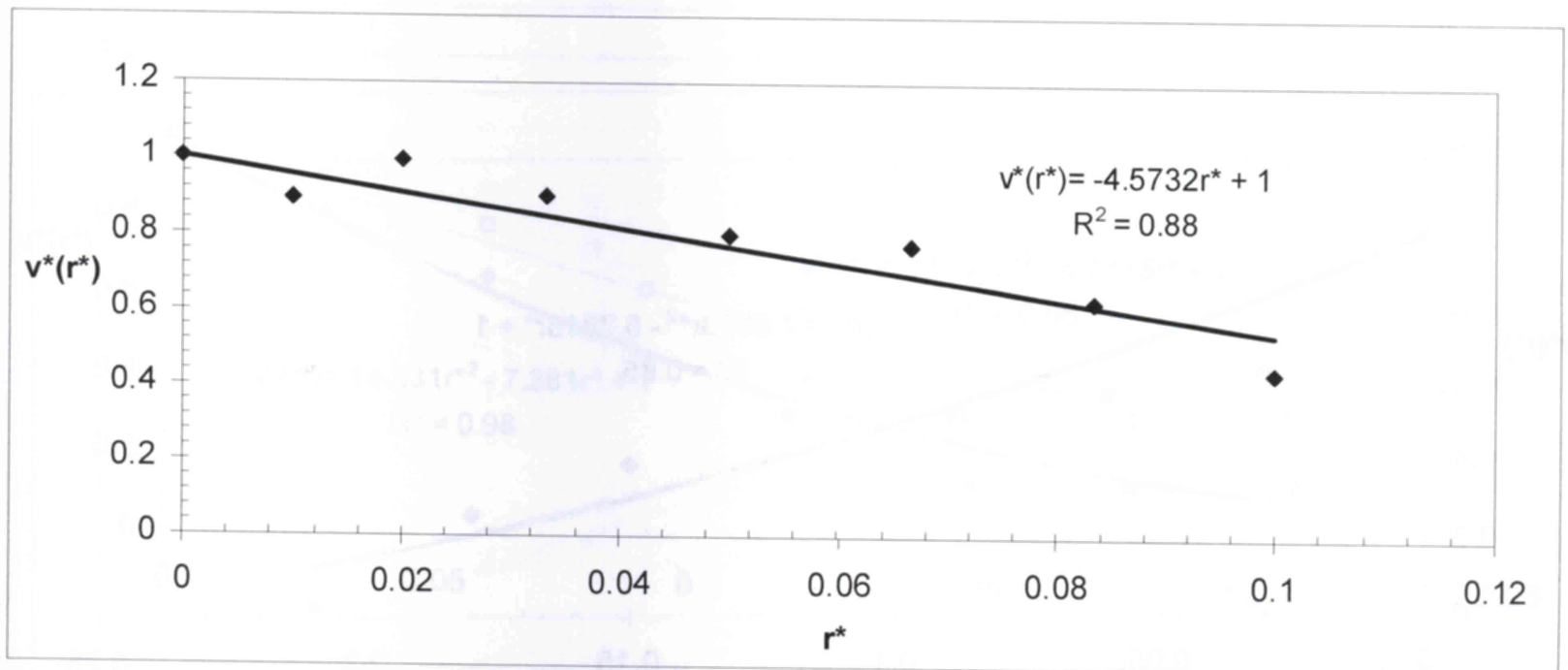

Figure 4.6: Calculated $v^{*}\left(r^{*}\right)$ vs. $r^{*}$ (corresponding to data in Table B-2 and particle spatial distribution in Figure 3.5) using $k=1.43$ (from [25]) for $25 \mu \mathrm{m}$ angular aluminum oxide blasted at $d=30 \mathrm{~mm}$ at pressure of $200 \mathrm{kPa}$ and flow rate of $2.83 \mathrm{~g} / \mathrm{min}$ using $0.76 \mathrm{~mm}$ round nozzle. - : Linear regression fit to calculated data $\left(\diamond: R^{2}=0.88\right)$. 


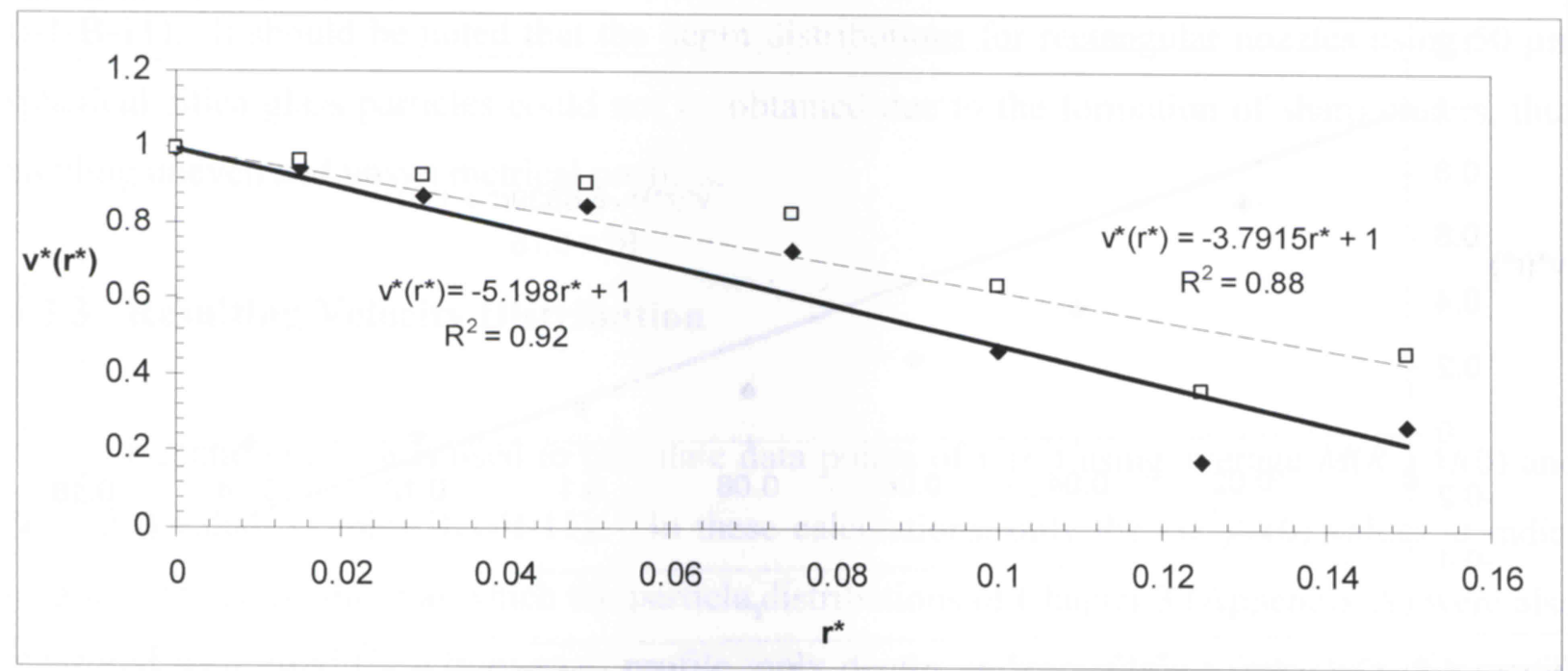

Figure 4.7: Calculated $v^{*}\left(r^{*}\right)$ vs. $r^{*}$ (corresponding to data in Table B-3 and particle spatial distribution in Figure 3.6) for $50 \mu \mathrm{m}$ silica glass beads blasted at $d=20 \mathrm{~mm}$ at pressure of $200 \mathrm{kPa}$ and flow rate of $1.17 \mathrm{~g} / \mathrm{min}$ using $0.76 \mathrm{~mm}$ round nozzle. - : Linear regression fit to calculated data $\left(\diamond: k=1.6, \mathrm{R}^{2}=0.92\right)$. ----: Linear regression fit to calculated data $(\square: k=$ 2.7, $\left.\mathrm{R}^{2}=\mathbf{0 . 8 8}\right)$.

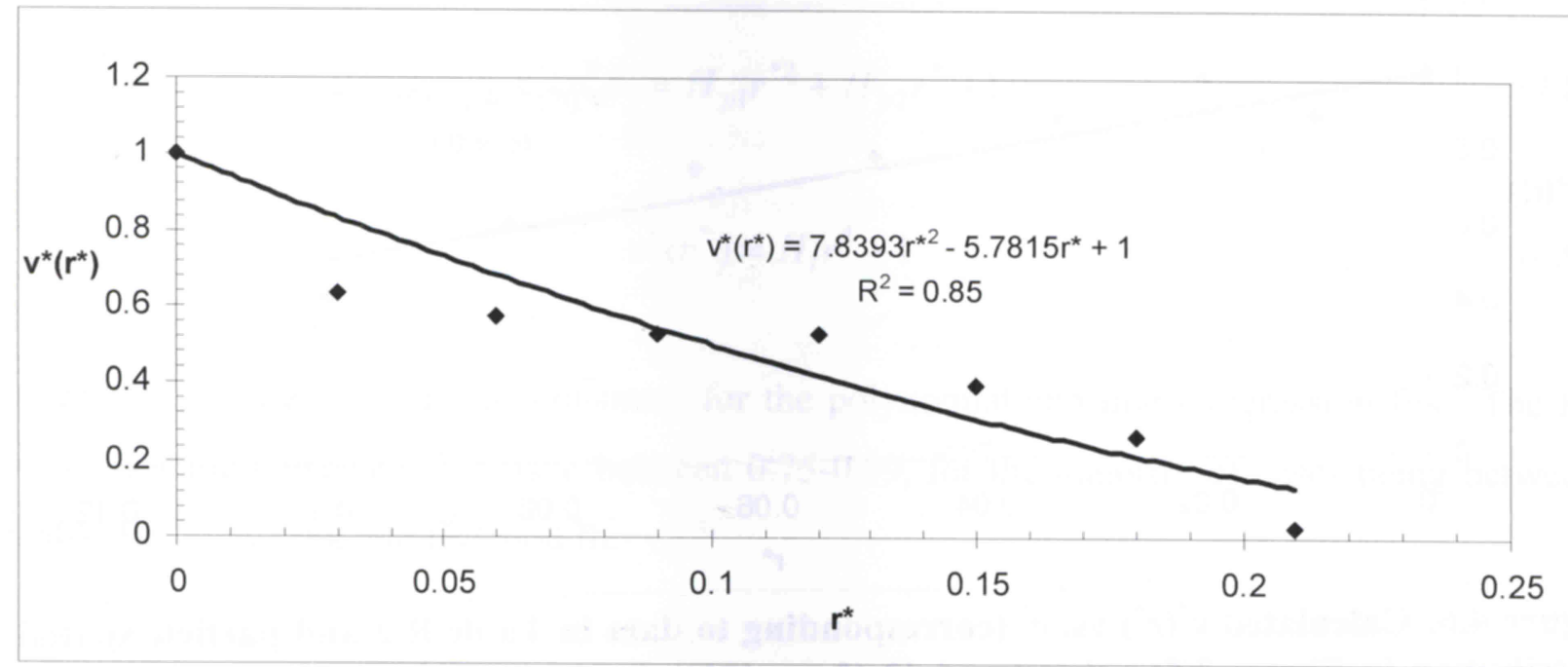

Figure 4.8: Calculated $v^{*}\left(r^{*}\right)$ vs. $r^{*}$ (corresponding to data in Table B-4 and particle spatial distribution in Figure 3.7) using $k=1.43$ (from [25]) for $25 \mu \mathrm{m}$ angular aluminum oxide blasted at $d=10 \mathrm{~mm}$ at pressure of $200 \mathrm{kPa}$ and flow rate of $0.36 \mathrm{~g} / \mathrm{min}$ using $0.46 \mathrm{~mm}$ H.P. round nozzle. —: Polynomial regression fit to calculated data $\left(\diamond: R^{2}=0.85\right)$. 


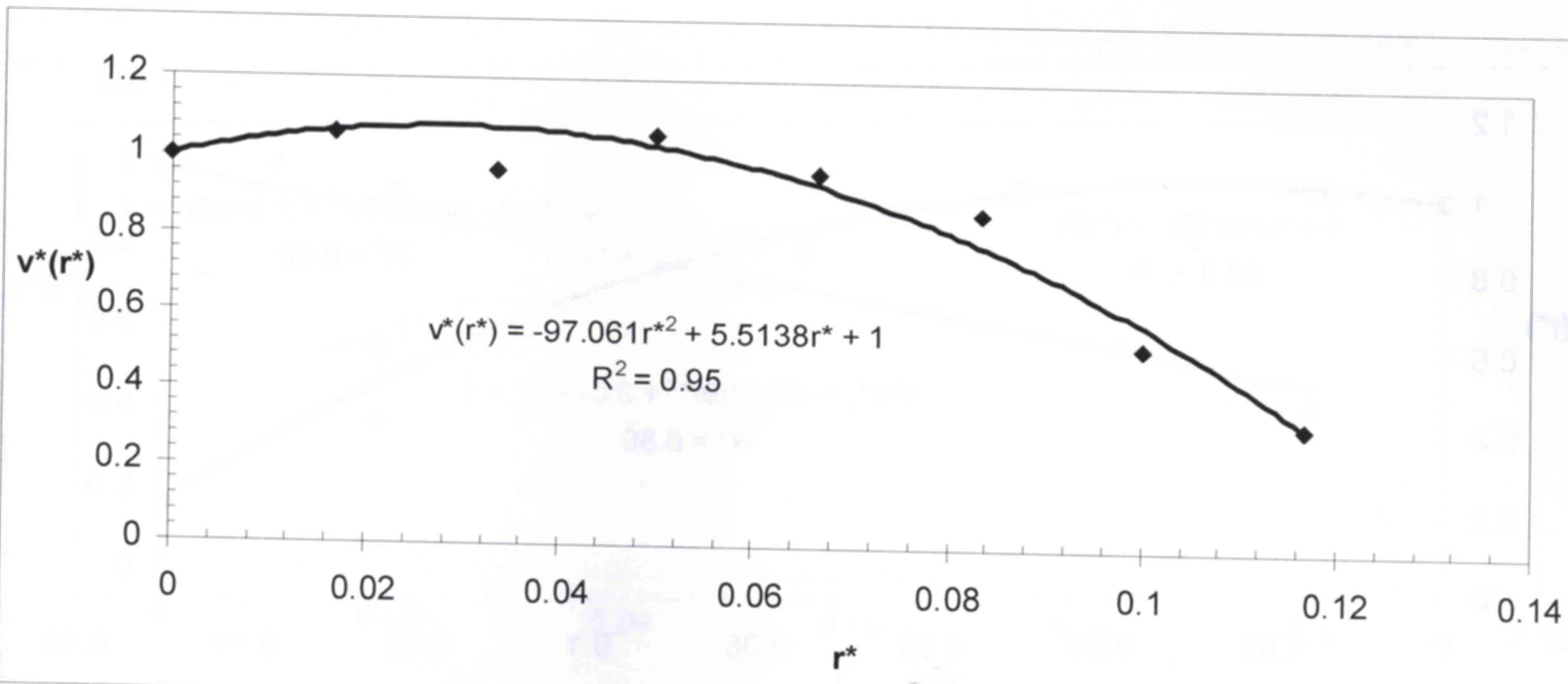

Figure 4.9: Calculated $v^{*}\left(r^{*}\right)$ vs. $r^{*}$ (corresponding to data in Table B-5 and particle spatial distribution in Figure 3.8) using $k=1.43$ (from [25]) for $25 \mu \mathrm{m}$ angular aluminum oxide blasted at $d=30 \mathrm{~mm}$ at pressure of $200 \mathrm{kPa}$ and flow rate of $4.75 \mathrm{~g} / \mathrm{min}$ using $1.5 \mathrm{~mm}$ H.P. round nozzle. - Polynomial regression fit to calculated data $\left(\diamond: R^{2}=0.95\right)$.

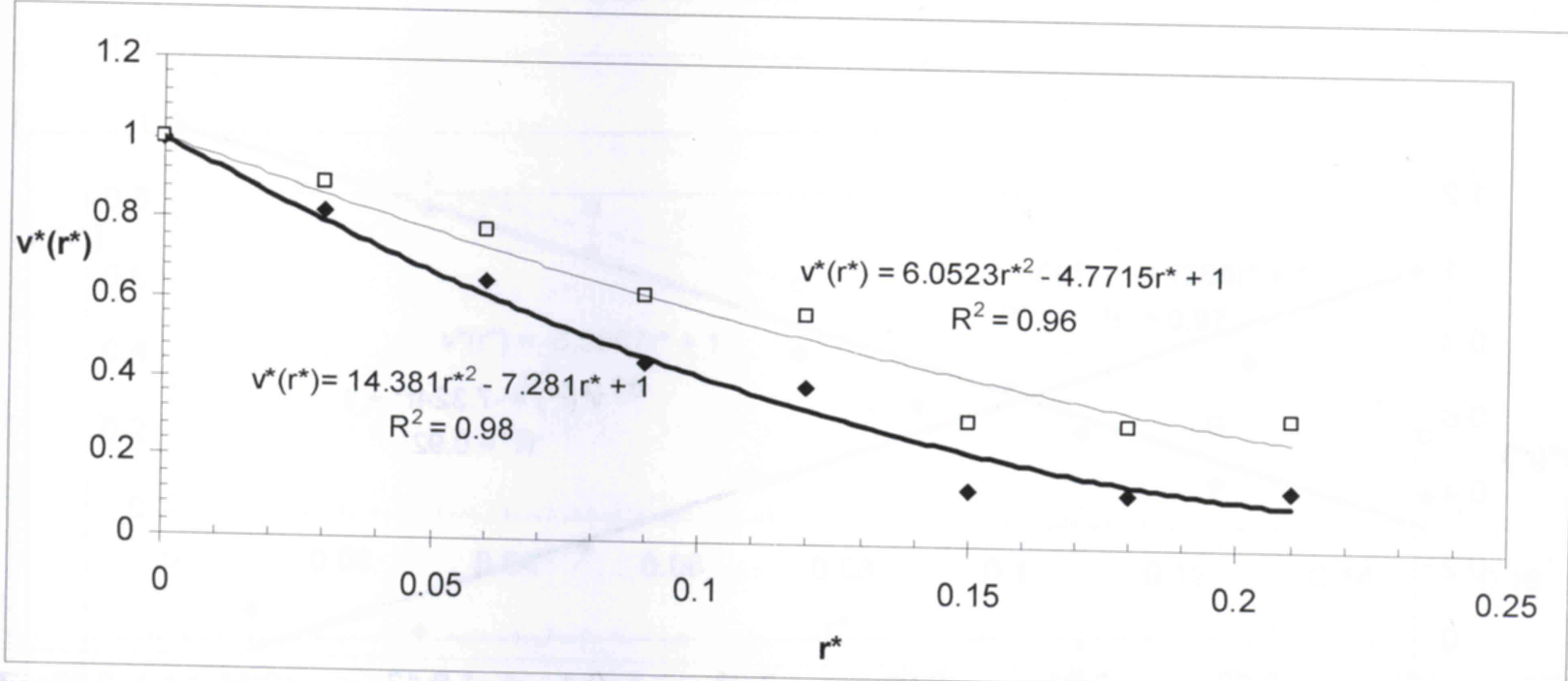

Figure 4.10: Calculated $v^{*}\left(r^{*}\right)$ vs. $r^{*}$ (corresponding to data in Table B-6 and particle spatial distribution in Figure 3.9) for $50 \mu \mathrm{m}$ silica glass beads blasted at $d=10 \mathrm{~mm}$ at pressure of $200 \mathrm{kPa}$ and flow rate of $0.23 \mathrm{~g} / \mathrm{min}$ using $0.46 \mathrm{~mm}$ H.P. round nozzle. -: Polynomial regression fit to calculated data $\left(\bullet: k=1.6, \mathrm{R}^{2}=0.98\right)$. ----: Polynomial regression fit to calculated data $\left(\square: k=2.7, \mathrm{R}^{2}=0.96\right)$. 


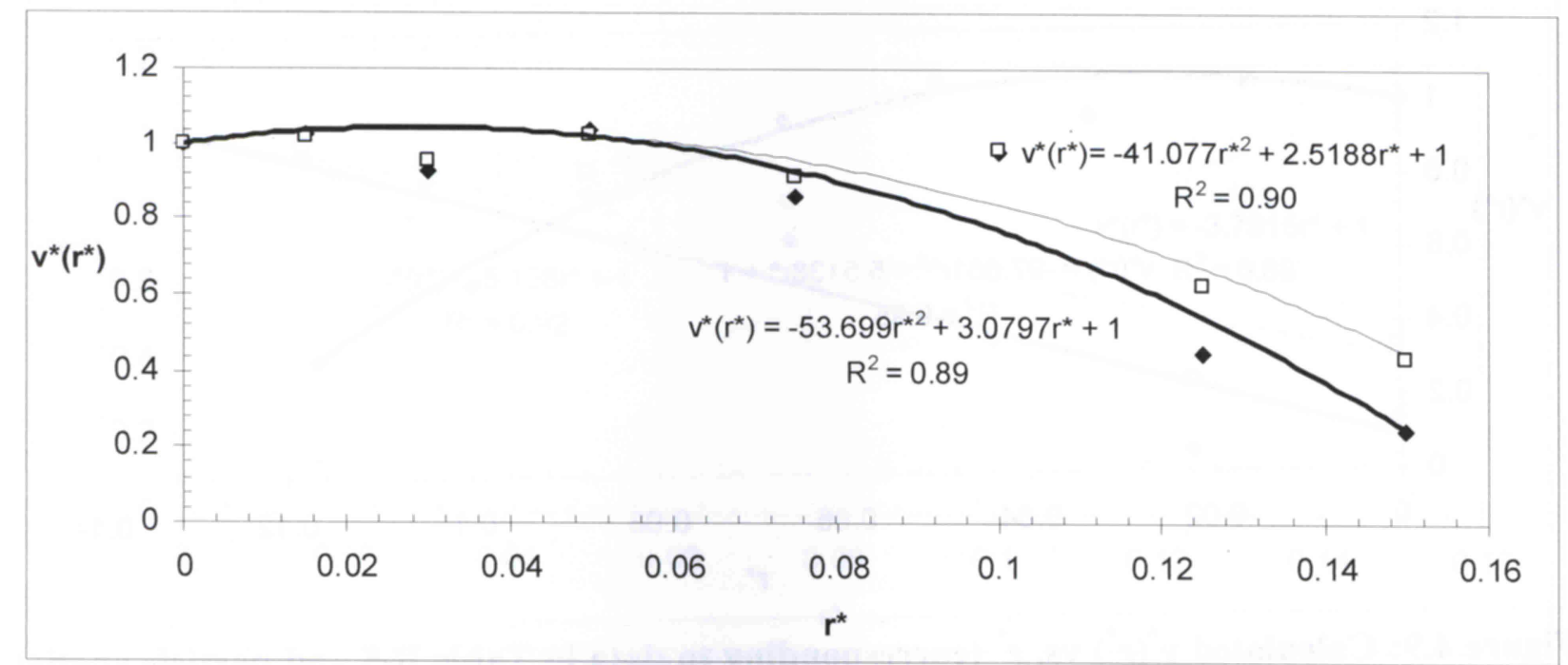

Figure 4.11: Calculated $v^{*}\left(r^{*}\right)$ vs. $r^{*}$ (corresponding to data in Table B-7 and particle spatial distribution in Figure 3.10) for $50 \mu \mathrm{m}$ silica glass beads blasted at $d=20 \mathrm{~mm}$ at pressure of $200 \mathrm{kPa}$ and flow rate of $1.31 \mathrm{~g} / \mathrm{min}$ using $1.5 \mathrm{~mm}$ H.P. round nozzle. - : Polynomial regression fit to calculated data $\left(\triangleleft: k=1.6, \mathrm{R}^{2}=0.89\right)$. ----: Polynomial regression fit to calculated data $\left(\square: k=2.7, \mathrm{R}^{2}=0.90\right)$.

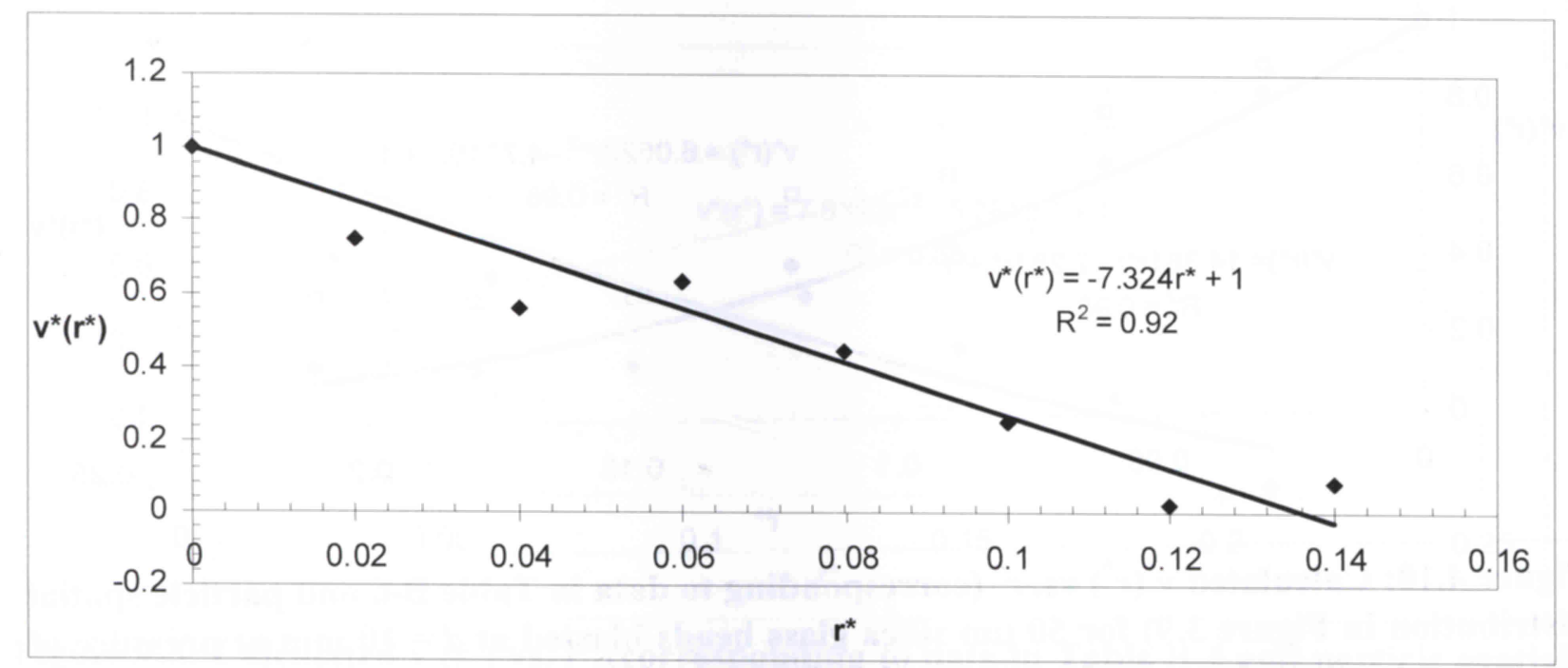

Figure 4.12: Calculated $v^{*}\left(r^{*}\right)$ vs. $r^{*}$ (corresponding to data in Table B-8a and particle spatial distribution in Figure 3.11) using $k=1.44$ (Figure 4.3) for $10 \mu \mathrm{m}$ angular aluminum oxide blasted at $d=30 \mathrm{~mm}$ at pressure of $300 \mathrm{kPa}$ and flow rate of $4.20 \mathrm{~g} / \mathrm{min}$ using 0.76 $\mathrm{mm}$ round nozzle. - - : Linear regression fit to calculated data $\left(\diamond: R^{2}=0.92\right)$. 


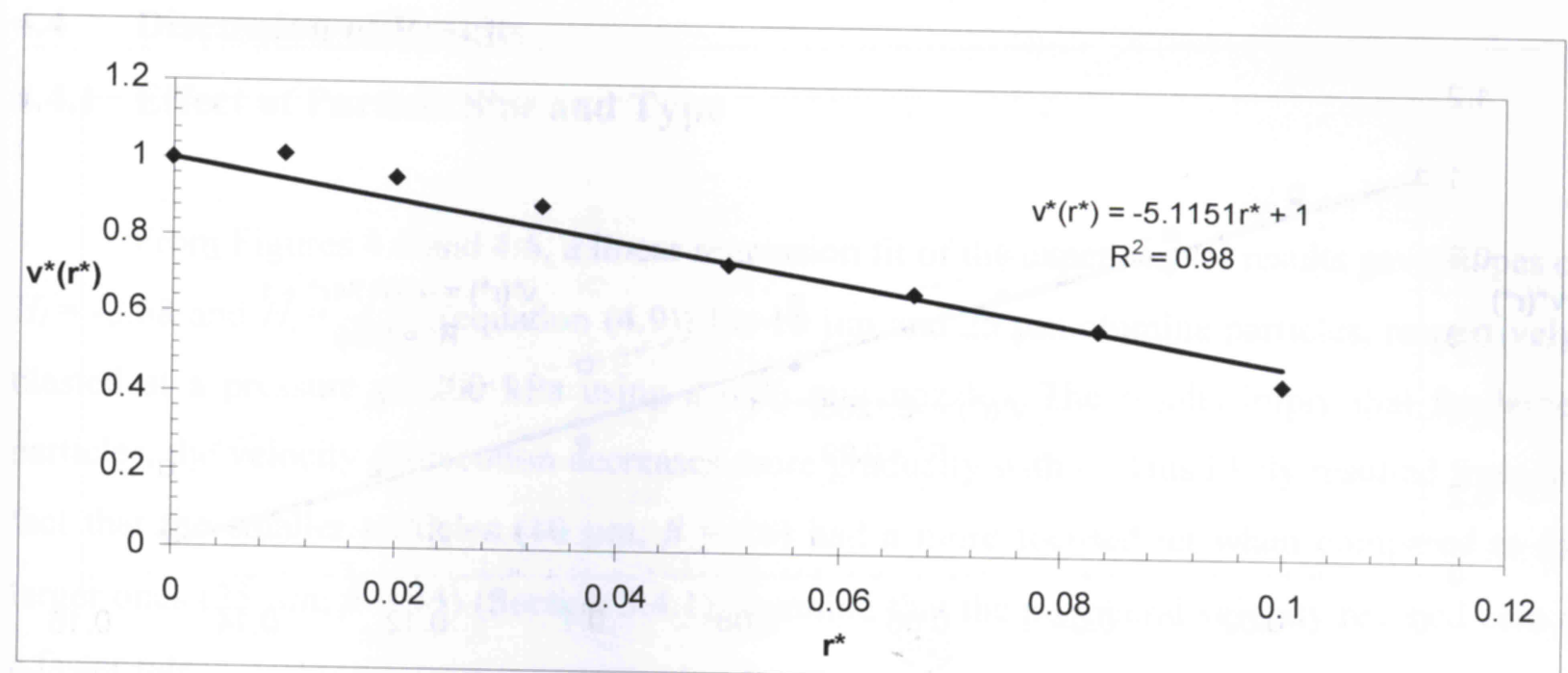

Figure 4.13: Calculated $v^{*}\left(r^{*}\right)$ vs. $r^{*}$ (corresponding to data in Table B-9a and particle spatial distribution in Figure 3.12) using $k=1.59$ (Figure 4.4) for $25 \mu \mathrm{m}$ angular aluminum oxide blasted at $d=30 \mathrm{~mm}$ at pressure of $300 \mathrm{kPa}$ and flow rate of $3.10 \mathrm{~g} / \mathrm{min}$ using 0.76 $\mathrm{mm}$ round nozzle. —: Linear regression fit to calculated data $\left(\bullet: R^{2}=0.98\right)$.

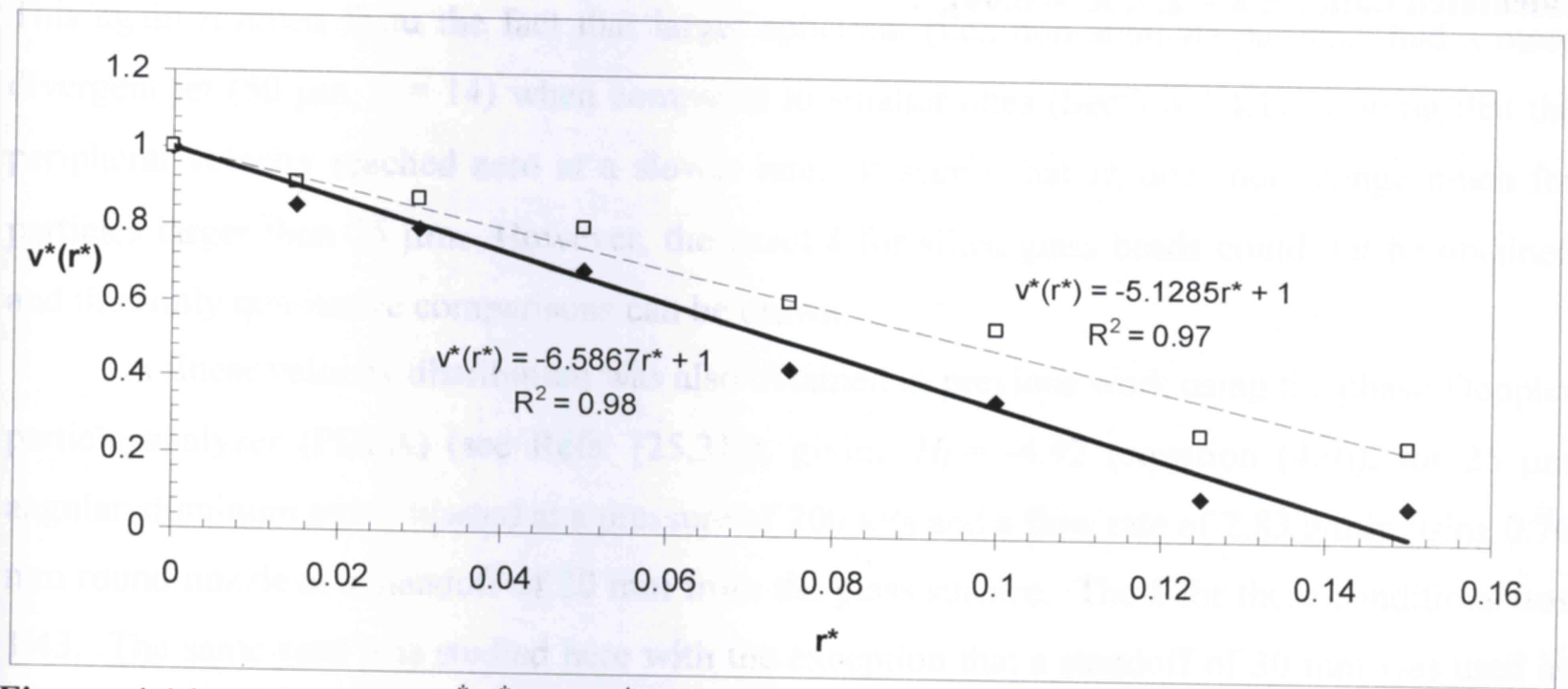

Figure 4.14: Calculated $v^{*}\left(r^{*}\right)$ vs. $r^{*}$ (corresponding to data in Table B-10 and particle spatial distribution in Figure 3.13) for $50 \mu \mathrm{m}$ silica glass beads blasted at $d=20 \mathrm{~mm}$ at pressure of $300 \mathrm{kPa}$ and flow rate of $1.34 \mathrm{~g} / \mathrm{min}$ using $0.76 \mathrm{~mm}$ round nozzle. - : Linear regression fit to calculated data $\left(\bullet: k=1.6, \mathrm{R}^{2}=0.98\right)$. ----: Linear regression fit to calculated data $\left(\square: k=2.7, \mathrm{R}^{2}=0.97\right)$. 


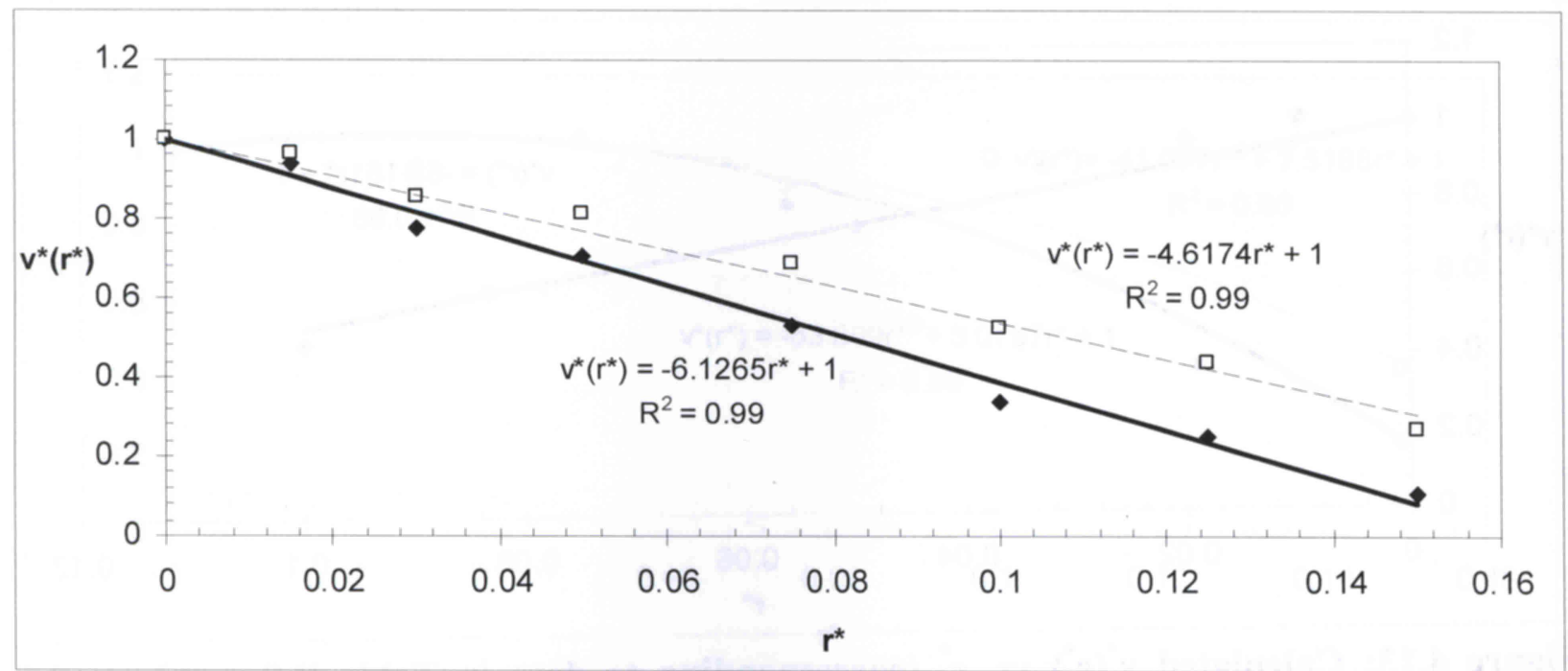

Figure 4.15: Calculated $v^{*}\left(r^{*}\right)$ vs. $r^{*}$ (corresponding to data in Table B-11 and particle spatial distribution in Figure 3.6) for $50 \mu \mathrm{m}$ silica glass beads blasted at $d=20 \mathrm{~mm}$ at pressure of $200 \mathrm{kPa}$ and flow rate of $0.59 \mathrm{~g} / \mathrm{min}$ using $0.76 \mathrm{~mm}$ round nozzle. - : Linear regression fit to calculated data $\left(\diamond: k=1.6, \mathrm{R}^{2}=0.99\right)$. ----: Linear regression fit to calculated data $\left(\square: k=2.7, \mathrm{R}^{2}=0.99\right)$. 


\subsection{Discussion of Results \\ 4.4.1 Effect of Particle Size and Type}

From Figures 4.5 and 4.6, a linear regression fit of the experimental results gave slopes of $H_{l}=-8.68$ and $H_{l}=-4.57$ (equation (4.9)) for $10 \mu \mathrm{m}$ and $25 \mu \mathrm{m}$ alumina particles, respectively, blasted at a pressure of $200 \mathrm{kPa}$ using a $0.76 \mathrm{~mm}$ nozzle. The results imply that for larger particles, the velocity distribution decreases more gradually with $r$. This likely resulted from the fact that the smaller particles $(10 \mu \mathrm{m}, \beta=16)$ had a more focused jet when compared to the larger ones ( $25 \mu \mathrm{m}, \beta=15)$ (Section 3.4.1), meaning that the peripheral velocity reached zero at a faster rate.

From Figure 4.7, for $50 \mu \mathrm{m}$ silica glass beads blasted at a pressure of $200 \mathrm{kPa}$ using a $0.76 \mathrm{~mm}$ nozzle, the average $H_{l}=-4.49$ (obtained from the two slopes for the upper and lower bounds of $k$ ), which was slightly smaller than $H_{l}=-4.57$ obtained for $25 \mu \mathrm{m}$ alumina particles. This again resulted from the fact that larger spherical (i.e., non-angular) particles had a more divergent jet (50 $\mu \mathrm{m}, \beta=14$ ) when compared to smaller ones (Section 3.4.1) meaning that the peripheral velocity reached zero at a slower rate. It seems that $H_{l}$ does not change much for particles larger than $25 \mu \mathrm{m}$. However, the exact $k$ for silica glass beads could not be obtained and thus only qualitative comparisons can be drawn.

A linear velocity distribution was also obtained in previous work using the phase-Doppler particle analyzer (PDPA) (see Refs. [25,31]), giving $H_{l}=-4.92$ (equation (4.9)), for $25 \mu \mathrm{m}$ angular aluminum oxide blasted at a pressure of $200 \mathrm{kPa}$ and a flow rate of $2.83 \mathrm{~g} / \mathrm{min}$ using 0.76 $\mathrm{mm}$ round nozzle at a standoff of $20 \mathrm{~mm}$ from the glass surface. The $k$ for these conditions was 1.43. The same case was studied here with the exception that a standoff of $30 \mathrm{~mm}$ was used as opposed to $20 \mathrm{~mm}$ (Figure 4.6), and a linear velocity distribution was obtained with $H_{l}=-4.57$, which differs from the slope for $d=20 \mathrm{~mm}$ obtained in Ref. [25] by 7\%. These comparisons can be made since $H_{l}$ is independent of $d$ (refer to Section 4.4.4) and since the particles all decelerate by the same amount over the extra $10 \mathrm{~mm}$ for the case studied presently, and thus the velocity distribution should not be affected. Hence, the present method of obtaining the velocity distribution across the jet seems to match that of using the PDPA. Similar linear velocity distributions across the jet have also been observed elsewhere [18,32]. 


\subsubsection{Effect of Nozzle Size}

From Figures 4.8-4.11, a non-linear velocity distribution was obtained for the $25 \mu \mathrm{m}$ alumina and $50 \mu \mathrm{m}$ silica glass beads blasted at a pressure of $200 \mathrm{kPa}$ using $0.46 \mathrm{~mm}$ H.P. and $1.5 \mathrm{~mm}$ H.P nozzles. For larger nozzles, the velocity distribution initially decreased gradually with $r$ (maintaining a slope close to 0 ), followed by a sharp decrease for higher $r$ (Figures 4.9 and 4.11). This may result from the fact that in a larger nozzle, the particles are less constrained near the center of the jet because inter-particle spacing is larger. For higher $r$, since the jet was more focused in larger nozzles (Section 3.4.2.1), the slope began to decrease more sharply. For smaller nozzles, the velocity distribution decreased in an exponential manner with $r$, leveling off for higher $r$ (Figures 4.8 and 4.10). This can result from the fact that in a smaller nozzle, the particles are more constrained near the center of the jet because inter-particle spacing is lower. For higher $r$, since the jet is more divergent for smaller nozzles (Section 3.4.2.1), the slope begins to level off. Similar non-linear velocity distributions have been reported elsewhere $[33,34]$.

\subsubsection{Effect of Pressure}

From Figures 4.12 and 4.13, a linear regression fit of the experimental results gave slopes of $H_{l}=-7.32$ and $H_{l}=-5.12$ (equation (4.9)) for $10 \mu \mathrm{m}$ and $25 \mu \mathrm{m}$ alumina particles, respectively, blasted at a pressure of $300 \mathrm{kPa}$ using a $0.76 \mathrm{~mm}$ nozzle, with $\beta$ values of 20 and 17 (Table 3.1), respectively. The $H_{l}$ values for the $200 \mathrm{kPa}$ pressure under otherwise identical conditions were found to be -8.68 and -4.57 (Figures 4.5 and 4.6), with $\beta$ values of 16 and 15 (Table 3.1), respectively. Thus, for the $25 \mu \mathrm{m}$ alumina particles, $H_{l}$ increased with pressure, implying that the velocity distribution decreased more sharply with $r$ since the jet was more focused for higher pressure for this particle type (Section 3.4.3). However, for the $10 \mu \mathrm{m}$ alumina particles, $H_{l}$ slightly decreased with pressure, implying that the velocity distribution decreased more gradually with $r$ even though the jet was more focused for higher pressure for this particle type (Section 3.4.3), and $k$ decreased with increasing pressure from 1.57 (Figure 4.2) to 1.44 (Figure 4.3), both of which should increase $H_{l}$. This difference could be attributed to the 
poor linear fit of equation (4.9) to experimentally obtained values in Figure 4.5 due to effect of particle interference affecting corresponding $M\left(R^{*}\right) / M(0)$ measurements in Figure 3.4.

For the $50 \mu \mathrm{m}$ silica glass beads blasted at a pressure of $300 \mathrm{kPa}$ using a $0.76 \mathrm{~mm}$ nozzle, a linear regression fit of the experimental results gave an average slope of $H_{l}=-5.89$ (Figure 4.14), with a $\beta$ value of 12 (Table 3.1). The average $H_{l}$ value for the $200 \mathrm{kPa}$ pressure under otherwise identical conditions was found to be -4.49 (Figure 4.7 ), with a $\beta$ value of 14 (Table 3.1). Thus, average $H_{l}$ increased with pressure implying that the velocity distribution decreased more sharply with $r$ although the jet was more divergent for higher pressure for this particle type (Section 3.4.3). However, for the $50 \mu \mathrm{m}$ silica glass beads, the $k$ likely decreases with higher pressure (i.e., velocity) because more of the kinetic energy of the particles is dissipated via particle breakage [30] and not towards erosion, as explained in Section 4.3.1, thus increasing $H_{l}$.

\subsubsection{Effect of Standoff Distance and Exposure Times}

Since the data for all the cases was normalized with respect to the jet center and nondimensionalized with respect to the standoff (i.e., plotted against $r^{*}=r / d$ ) (Figures 4.5-4.15), the velocity distribution was relatively insensitive to standoff distance, for low standoffs (which is the case here). This is supported by the fact that the same $H_{l}$ was obtained in Ref. [25] as for the case studied in Figure 4.6, although a different standoff was used, for otherwise the same conditions $\left(H_{l}=-4.92, d=20 \mathrm{~mm}\right.$ and $H_{l}=-4.57, d=30 \mathrm{~mm}$ in Figure 4.6 and Ref. [25], respectively) (see Section 4.4.1). Thus, since $H_{l}$ is independent of $d$, it follows that the velocity distribution was independent of $d$ (equation (4.9)), and thus the particles follow straight trajectories from the nozzle to surface [15].

Finally, it should be noted that the velocity distribution, as expected, was independent of the exposure time from $M\left(R^{*}\right) / M(0)$ and the erosion time from $\delta\left(r^{*}\right) / \delta(0)$ (equation (4.7)), because all exposure times were far greater than the time required to reach steady state.

\subsubsection{Effect of Particle Mass Flow Rate}

$M\left(R^{*}\right) / M(0)$ can depend on the mass flow rate at low values of $r$ for the cases in Figures 3.4 and 3.11 (Section 3.4.5), because of the possibility of particle interference between incoming 
particles and those rebounding from the collection cylinder face (Section 3.4.7). Thus, it can affect the velocity calculations for cases corresponding to Figures 4.5 and 4.12. In addition, $k$ can also depend on the mass flow rate if it is high, because of the interference between incident and rebounding from glass surface particles, which can also affect the velocity calculations. From Figures 4.7 and 4.15, for $50 \mu \mathrm{m}$ glass beads blasted at a pressure of $200 \mathrm{kPa}$ using 0.76 $\mathrm{mm}$ round nozzle, halving the particle mass flow rate (i.e., from $1.17 \mathrm{~g} / \mathrm{min}$ to $0.59 \mathrm{~g} / \mathrm{min}$ ) caused the average $H_{l}$ to slightly increase from -4.49 to -5.37 . However, since for the silica glass beads, exact values of $k$ could not be obtained, only qualitative comparisons can be drawn.

\subsubsection{Advantages over Other Methods and Sources of Error}

The present method of obtaining the velocity distribution across the jet represents a relatively straightforward low cost alternative to the use of PDPA [25,31]. The PDPA method, suffers from the fact the laser must be realigned often, it is very sensitive, and the results often depend on the sampling control volume used, which is often too large for the present particle size range. On the other hand, the present method can only be used to obtain the velocity distribution, and not the actual magnitude of velocity (which can be obtained by using the PDPA or the opto-electronic flight timer method of Refs. [1,15]). However, the attainment of the velocity distribution is more important since it determines the actual profile shape. In addition, the present method is more complex since two pieces of equipment (i.e., the microblaster setup and the profilometer) and three sets of experiments (particle catching, velocity exponent, and profile) must be performed in order to obtain the results. Nevertheless, the velocity exponent and profile measurements are routine in many assessments of erosion resistance, and the cost of the PDPA system alone justifies the use of the present method.

Nozzle misalignments, complexity of the experimental apparatus, and particle interference are possible sources of error for the present method of obtaining the velocity distribution. Nozzle misalignments can cause variability in the experimental data. In addition, since several pieces of equipment were used (i.e., the microblaster setup and the profilometer) to obtain one set of data points, the measurement errors could be compounded to cause further variability in the experimental data. Finally, the effect of particle interference affecting 
$M\left(R^{*}\right) / M(0)$ measurements for two cases discussed in Section 3.4.7 can affect the accuracy in calculating the velocity distribution via equation (4.7) (Figures 4.5 and 4.12). 


\section{Chapter 5 Analytical Model of the Interference Effects in a Divergent Jet at High Flux Conditions}

\subsection{Motivation}

As was mentioned in Section 2.1, in solid particle erosion testing, the dependency of the erosion rate on incident particle flux can be attributed to the effects of interference between impacting particles, and those rebounding from the surface (e.g., [4,8-10,12]). These often neglected effects can significantly limit the erosive potential at the surface [3] and thus also have important implications for abrasive jet micromachining applications. In Chapter 2, analytical models capable of predicting particle interference effects in a non-diverging (i.e., cylindrical) jet having uniform particle spatial and velocity distributions were presented. However, the measurements of Chapters 3 and 4 showed that, in systems typical of abrasive jet micromachining, particle velocity and spatial distributions are not uniform. In this chapter, an analytical model of the interference between incident and rebounding particles in the more realistic divergent jet case will be derived.

In Chapter 2, Model B (Modified Anand et al. model) best matched the results of the computer simulation because both it and the simulation were based on derivations of particle interference at steady state, and included the effects of stream density (i.e., particle spacing), radial symmetry, and particle-to-surface collision energy losses. Therefore, this model is chosen as a starting point, and will be modified to include these effects for a divergent jet, which has non-uniform particle spatial and velocity distributions. This model will be used to first derive the probability of an incident particle surviving to a particular surface region, and then the entire surface, without colliding, at normal incidence $\left(\alpha=90^{\circ}\right)$. Finally, analytical expressions will be derived that give the maximum possible incident flux in order for interference effects to be limited to a certain level. The results will then be compared to the updated modified computer simulation of Ref. [13]. It should be noted that a number of equations derived in previous chapters will be repeated here in order to maintain continuity. 


\subsection{Modification of Method of Anand et al. [9]}

\subsubsection{Modifications to Take into Account Jet Divergence and Particle Spatial Distribution}

In order to simplify the analysis, only normal incidence is considered $\left(\alpha=90^{\circ}\right)$. The normal incidence case is of particular importance since it is the situation in which particle interference is expected to be maximum, since the size of the collision zone (the volume over which collisions can occur) spans the full standoff distance, where multiple sets of collisions are possible, and thus it represents the worst case scenario [10,12,13].

As was mentioned in Chapter 2, in Ref. [8] as well as [9], the locations of spherical particles of radius $r_{p}$ within a non-divergent jet cross section of area $\pi r_{n}{ }^{2}$, where $r_{n}$ is the nozzle radius, are assumed to be random; i.e., particles are no more likely to appear at a given ring area, $2 \pi r d r$, in the jet than any other (Figure 5.1). However, the geometry and symmetry of the jet cross section imply that more particles can fit in the outer rings in Figure 5.1, than at, for example, the center of the jet $(r=0)$, an assumption built into the computer simulations of Refs. $[10,12,13]$. In addition, the probability of finding a particle decreases with $r$, meaning that less particles are actually present in the outer rings in Figure 5.1, than at, for example, rings closer to the jet center. This also means that the average space between particles is larger at a higher $r$. Finally, since the stream is divergent, the size of a particular stream cross section varies with the standoff distance, $d$, reaching its maximum at the surface, which is assumed flat (Figure 5.1). In the present analysis, all of this is accounted for by incorporating the Weibull particle spatial distribution [25], which has been found in Chapter 3 to accurately describe the particle spatial distribution for a wide variety of nozzles used in micro-abrasive blasting. However, it should be noted that, in general, any distribution can be used in the model derivation.

The jet cross section at the surface defines the target area for the arriving particle jet. Let $r=r_{s u}$ be the arbitrary radial position at which an initial arriving particle impacts the target surface, arriving with a velocity $v_{a}\left(r_{s u}\right)$ and an angle $\phi\left(r_{s u}\right)$, measured from the nozzle axis, where the subscript $s u$ indicates that $r$ is measured at the surface and subscript $a$ stands for arrival. It is assumed that the particles are evenly distributed along each arrival trajectory, arriving one at a time, in straight trajectories, each separated by an average distance $v_{a}\left(r_{s u}\right) / \Omega_{a}$, measured along the trajectory (Figure 5.1). 
From Chapter 2 and Refs. [10,12], the incident dimensionless stream density, $\rho_{s}$ (the ratio of the total volume of particles in the jet to the volume of the jet itself [12]), is used to quantify all the jet variables presented in Figure 5.1 into one dimensionless quantity. From Figure 5.1, the jet volume is defined by the frustum of a right circular cone, with a base and tip radius of $r_{\max }$ and $r_{n}$, respectively. Hence, the dimensionless incident stream density is defined as [12]:

$$
\rho_{s}=\frac{\frac{4}{3} \pi r_{p}^{3} \frac{\Omega_{a}}{v_{a}(r=0)} d}{\frac{\pi d\left(r_{n}^{2}+r_{n}\left(d \tan \lambda+r_{n}\right)+\left\{d \tan \lambda+r_{n}\right\}^{2}\right)}{3}}=\frac{4 r_{p}^{* 3}}{3 r_{n}{ }^{* 2}+3 r_{n}{ }^{*} d^{*} \tan \lambda+d^{* 2} \tan ^{2} \lambda}
$$

where $\lambda$ is the jet divergence angle (Figure 5.1) and where $r_{p}{ }^{*}, r_{n}{ }^{*}$ and $d^{*}$ are the dimensionless particle radius, nozzle radius, and standoff distance, respectively; i.e., the number of particles leaving the nozzle in the time it takes a particle to travel a distance equal to $r_{p}, r_{n}$ or $d$ [12]:

$$
\begin{aligned}
& r_{p}{ }^{*}=\frac{\Omega_{a} r_{p}}{v_{a}(r=0)} \\
& r_{n}{ }^{*}=\frac{\Omega_{a} r_{n}}{v_{a}(r=0)} \\
& d^{*}=\frac{\Omega_{a} d}{v_{a}(r=0)}
\end{aligned}
$$

where $\Omega_{a}$ is the particle nozzle launch frequency (particles/s) and $v_{a}(r=0)$ is the particle arrival velocity along the jet center (i.e., $r=0$ ), and is assumed to be constant along $d$ after being launched from the nozzle. 


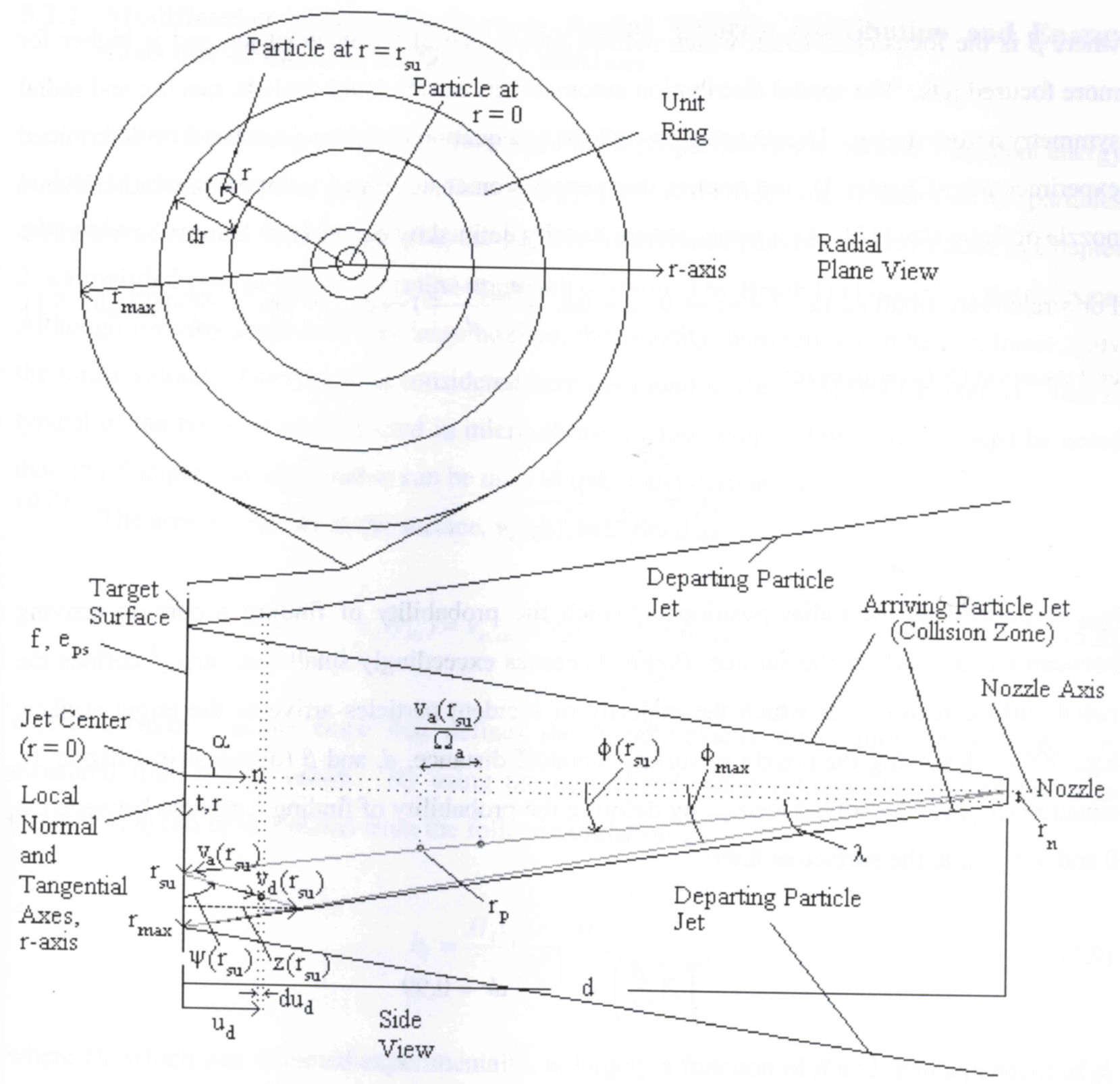

Figure 5.1: Side and radial plane views of a divergent particle jet impacting the target surface. Initial arriving particle impacts the surface at $r=r_{s u}$.

The present model incorporates the Weibull particle spatial distribution (or pdf), $P(r)$, which was found to describe the experimental situation well in Chapter 3, [25]:

$$
P(r)=2\left(\frac{\beta}{d}\right)^{2} r e^{-\left(\frac{\beta \cdot r}{d}\right)^{2}}
$$


where $\beta$ is the focus coefficient, which defines divergence of the incident jet, and is higher for more focused jets. The spatial distribution automatically accounts for particle spacing and radial symmetry across the jet. The distribution defined by equation (5.5) requires that $\beta$ be determined experimentally (Chapter 3), and implies that particles emanate from a point source, rather than a nozzle of finite size [13]. As a result, stream density defined by equation (5.1) must be modified.

For a relatively small $r_{n}$, i.e., for $r_{n} \rightarrow 0, \lambda=\tan ^{-1}\left(\frac{r_{\max }-r_{n}}{d}\right) \rightarrow \phi_{\max }=\tan ^{-1}\left(\frac{r_{\max }}{d}\right)$ (Figure 5.1), and equation (5.1) reduces to:

$$
\rho_{s}=\frac{4 r_{p}^{* 3}}{d^{* 2} \tan ^{2} \phi_{\max }}
$$

$r_{\max }$ is defined as the radial position at which the probability of finding a particle arriving between $r$ and $r+\mathrm{d} r$ at the surface, $P(r) d r$, becomes exceedingly small (i.e., $\pi r_{\max }{ }^{2}$ defines the radial surface region over which the majority of incident particles arrive to the target surface, e.g., 99\%). Knowing the nozzle to surface standoff distance, $d$, and $\beta$ (obtained in Chapter 3), equation (5.5) can be solved for $r_{\max }$ by defining the probability of finding a particle between $r=$ 0 and $r=r_{\max }$ at the surface as 0.99 :

$$
\begin{aligned}
& \int_{0}^{r_{\max }} 2\left(\frac{\beta}{d}\right)^{2} r e^{-\left(\frac{\beta \cdot r}{d}\right)^{2}} d r=0.99 \\
& r_{\max }=\sqrt{-\operatorname{In}(0.01)} \cdot \frac{d}{\beta}
\end{aligned}
$$

The remaining $1 \%$ of particles present at the surface between $r=r_{\max }$ and $r=\infty$ are assumed to not contribute to the incident flux, and thus to the overall interference effect. The results for individual $r_{\max }$ 's can be found in Table 5.1 for the different cases examined (Figures 5.3-5.10). 


\subsubsection{Modification to Include Particle Radial Velocity Distribution and Energy Loss due to Particle-to-Surface Collisions}

In erosion testing, it is likely that a significant proportion of the particle's incident energy is lost in damaging the surface. This energy loss will affect the trajectories of particles rebounding from the surface, and thus the particle interference patterns. As was done in Chapter 2, a simplified coefficient of restitution approach developed by Brach [11] is used in the analysis. Although for very small and very large nozzles, the velocity distribution can be non-linear, only the linear velocity distribution is considered here (as found in Ref. [25] and Chapter 4). This is typical of the types of nozzles used in micro-abrasive blast setups. However, it should be noted that, in principle, any distribution can be used in the model derivation.

The arrival velocity at the surface, $v_{a}\left(r_{s u}\right)$, is defined as:

$$
v_{a}\left(r_{s u}\right)=v_{a, s u}=v_{a}(r=0)+h_{l} r_{s u}
$$

where $h_{l}(\mathrm{~m} / \mathrm{m} \cdot \mathrm{s})$ is the slope that defines the linear velocity distribution decrease with $r$, measured from the jet center. By using the normalized radial velocity distribution found in Chapter $4, h_{l}$ can be calculated from the following relation:

$$
h_{l}=\frac{H_{l} v_{a}(r=0)}{d}
$$

where $H_{l}$, which was obtained experimentally, is largely a function of $\beta$ and is independent of $d$, assuming that $d$ is large enough so that aerodynamic effects are negligible [28] (Chapter 4). The arrival velocity, $v_{a, s u}$, can be divided into velocity components normal (n) and tangential (t) to the surface (Figure 5.1), $v_{a n, s u}=v_{a, s u} \cos \left(\phi_{s u}\right)$ and $v_{a t, s u}=v_{a, s u} \sin \left(\phi_{s u}\right)$, respectively, where

$$
\phi\left(r_{s u}\right)=\phi_{s u}=\tan ^{-1}\left(\frac{r_{s u}}{d}\right)
$$

is the angle that the nozzle axis makes with the arrival particle trajectory measured from the center of the nozzle to the surface at a particular $r_{s u}$ (Figure 5.1). Since at normal incidence the 
divergent jet is axis-symmetric, the 3-dimensional (3D) problem can be reduced to a 2dimensional (2D) one (Figure 5.1). As in Chapter 2, for the low friction case, when sliding during the impact with the target occurs, using the fact that impulse equals change in momentum, neglecting particle rotational rebound velocity before and after impact with the target and realizing that the arrival velocity at a particular $r_{s u}$ varies only with $\phi_{s u}$ and no other out-of-plane angle, it can be shown that $[10,11] v_{d n, s u}$ and $v_{d t, s u}$, the departing local 2D normal and tangential particle velocity components, are, respectively:

$$
\begin{gathered}
v_{d n, s u}=-e_{p s} v_{a n, s u} \\
v_{d t, s u}=f\left(v_{d n, s u}-v_{a n, s u}\right)+v_{a t, s u}=-f v_{a n, s u}\left(1+e_{p s}\right)+v_{a t, s u}
\end{gathered}
$$

where $f$ and $e_{p s}$ are, respectively, the friction coefficient and coefficient of restitution, for particle-to-surface collisions, and the subscript $d$ stands for departing. For the high friction case, when sticking during impact with the target occurs, $f$ in equation (5.12) is replaced by the critical impulse ratio, $\mu_{t}=2 v_{a t, s u} /\left[7 v_{a n, s u}\left(1+e_{p \mathrm{~s}}\right)\right][11]$, which is obtained by setting expressions for the final tangential velocities in the sliding solution equal to those obtained in the rolling solution (obtained from the conservation of angular momentum equation). The departing rotational velocity is ignored since the model only considers the first collision with the surface and neglects the rotational velocity in inter-particle collisions (since it is irrelevant due to the fact that the analytical model assumes that both the arriving and the departing particle is removed from the analysis after a collision takes place (Chapter 2, [9])). Using equations (5.11) and (5.12), the magnitude of the surface departing velocity including restitution effects at a particular $r_{s u}$ is

$$
v_{d, s u}=\sqrt{\left(v_{d n, s u}\right)^{2}+\left(v_{d t, s u}\right)^{2}}
$$

$\psi\left(r_{s u}\right)$ is the angle between the target surface and the departing trajectory at a particular $r_{s u}$, as shown in Figure 5.1, and is defined as:

$$
\psi\left(r_{s u}\right)=\psi_{s u}=\tan ^{-1}\left(\frac{v_{d n, s u}}{v_{d t, s u}}\right)
$$




\subsubsection{Probability of an Incident Particle Arriving to a Particular Surface Region without Colliding}

The collision zone, or the volume over which collisions can occur, is created by the intersection of arriving and departing particle jets $[8,9,10]$. For the present case, it is the volume formed by the cone of radius $r_{\max }$ and height $d$ (i.e., the volume formed by the arriving jet) (Figure 5.1 or 5.2). Let $z\left(r_{s u}\right)$ (henceforth referred to as $z_{s u}$ ) be the rebound length over which collisions can occur for the surface-departing particle trajectory after arriving to the surface at a particular ring located at $r_{s u}$, measured perpendicular to the surface (Figure 5.2). Since the jet is axis-symmetric, only the $2 \mathrm{D}$ case needs to be considered. $z_{s u}$ can be obtained by finding the intersection point between the line formed by the departing particle trajectory at the angle $\psi_{s u}$ (measured from the target surface) after a collision with the surface at a particular $r_{s u}$, and the line formed by an arriving particle trajectory at an angle $\phi_{\max }$ (measured from the nozzle axis) reaching $r_{\max }$ at the surface (Figure 5.2). It is assumed that the distance over which a particle slides or rolls during impact with the surface (Section 5.2.2) is negligible and thus it does not affect the $z_{s u}$ calculation. Using trigonometry from Figure 5.2 and the sine law:

$$
z_{s u}=\left(r_{\max }-r_{s u}\right) \cdot \frac{\sin \psi_{s u} \sin \left(\pi / 2-\phi_{\max }\right)}{\sin \left(\pi-\psi_{s u}-\left(\pi / 2-\phi_{\max }\right)\right)}=\left(r_{\max }-r_{s u}\right) \cdot \frac{\sin \psi_{s u} \cos \phi_{\max }}{\sin \left(\pi / 2-\psi_{s u}+\phi_{\max }\right)}
$$

Further, let $d$ be the arrival length over which collisions can occur for a surface-arriving particle trajectory after leaving the nozzle, measured perpendicular to surface (Figure 5.2).

The probability of collision between some incident particle and the departing particle (after impacting the surface at a particular $r_{s u}$ ) can be derived by considering the intersection between an incident particle trajectory and a departing particle trajectory at steady state [9]. Let $u_{d}$ be the distance traveled by a departing particle on a collision trajectory defined by the angle $\psi_{s u}$, after impacting the surface at a particular ring at $r_{s u}$, measured perpendicular to the surface, until it intersects an incident particle trajectory on the infinitesimal cross section of thickness $d u_{d}$ at a certain $r_{\text {coll,su }}$ from the jet center (Figure 5.2), where

$$
r_{\text {coll }, s u}=r_{s u}+\frac{u_{d}}{\tan \left(\psi_{s u}\right)}
$$




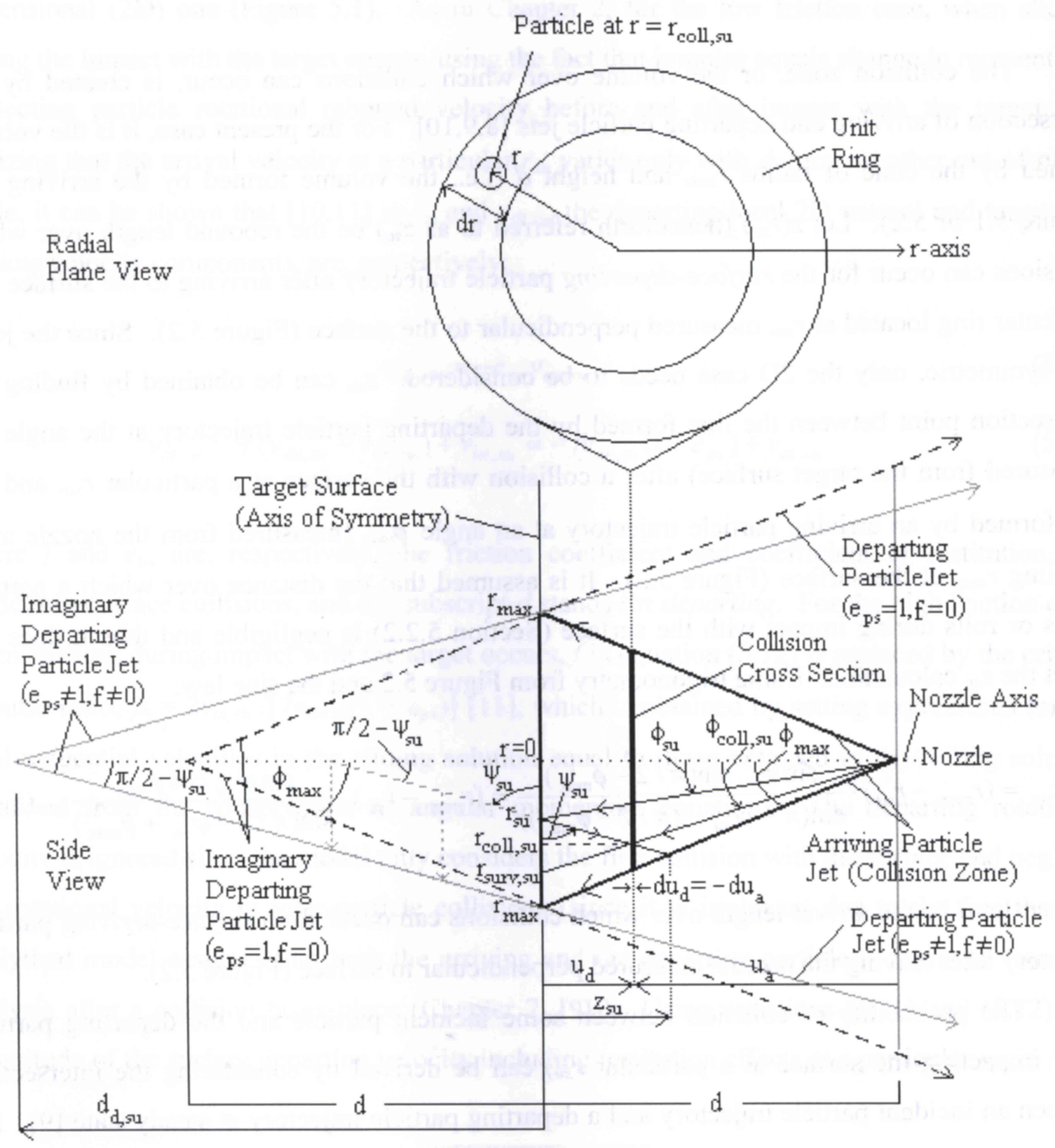

Figure 5.2: Collision between an arriving and a departing particle at $r=r_{\text {coll,su. }}$. The lefthand side of the diagram shows an imaginary departing jet plume for $e_{p s}=1, f=0$, for all $r_{s u}$ (broken lines), and for $e_{p s} \neq 1, f \neq 0$, for a specific $r_{s u}$ (solid lines). The right-hand side of the diagram shows the arriving jet plume (solid bold lines) along with the real departing jet plume for $e_{p s}=1, f=0$, for all $r_{s u}$ (broken lines), and for $e_{p s} \neq 1, f \neq 0$, for a specific $r_{s u}$ (solid lines), both extended from the imaginary side. The radial plane view shows the collision cross section. 
Further, let $u_{a}$ be the distance traveled by an arriving particle, on a collision trajectory defined by the angle $\phi_{c o l l, s u}=\tan ^{-1}\left(r_{c o l l, s u} / u_{a}\right)$, after leaving the nozzle, measured perpendicular to the surface, until it intersects a departing particle trajectory on the infinitesimal cross section of thickness $d u_{a}$, which has the same magnitude as $d u_{d}$, at a certain $r_{c o l l, s u}$ from the jet center (Figure 5.2). $r_{\text {coll,su }}$ is still defined by equation (5.16), however, with $d$ - $u_{a}$ in the place of $u_{d}$, since $u_{a}+u_{d}=d$ (Figure 5.2).

At steady state, the number of arriving and departing particles per unit time must be equal at any jet cross section at any distance $u_{d}$ above the surface or at any distance $u_{a}$ from the nozzle (i.e., $\Omega_{a}=\Omega_{d}$ ) [9], where $\Omega_{d}$ is the departing particle frequency (particles/s).

Let $\sigma=\pi\left(2 r_{p}\right)^{2}$ be a particle collision cross section [9], where $2 r_{p}$ is the minimum distance between two particles within a jet cross section spanning all possible collision zones. $\sigma$ is used to define the probability, $p_{\text {coll }}\left(r_{\text {coll,su }}\right)$, that a single arriving particle undergoes a collision with any single departing particle at $r_{s u}$ at the cross section $d u_{d}$, or equivalently, $d u_{a}$. The change in this probability, $d p_{\text {coll }}\left(r_{\text {coll,su }}\right)$, over the distance $d u_{d}$ or $d u_{a}$, can be derived in terms of $d N_{\text {tot,su }}\left(r_{s u}\right)$ (henceforth referred to as $d N_{t o t, s u}$ ), the change in the total number of departing and arriving particles per unit cross sectional area at the cross section $d u_{d}$ or $d u_{a}$ (i.e., at any $r_{\text {coll,su}}$ ), since both the departing and arriving particle fluxes contribute to the probability of collision between an arriving and a departing particle:

$$
d p_{\text {coll }}\left(r_{\text {coll }, s u}\right)=\left[1-p_{\text {coll }}\left(r_{\text {coll }, s u}\right)\right](\sigma) d N_{\text {tot }, s u}=\left[1-p_{\text {coll }}\left(r_{\text {coll }, s u}\right)\right]\left(4 \pi r_{p}{ }^{2}\right) d N_{\text {tot }, s u}
$$

where $\sigma d N_{\text {tot,su }}$ is the total collision cross section per unit area (the probability that the particles will collide on $d u_{d}$ or $d u_{a}$ ), and [1- $\left.p_{\text {coll }}(r)\right]$ is the probability that both the departing and arriving particle have not yet collided, and hence have reached the cross section $d u_{d}$ at $u_{d}$ or $d u_{a}$ at $u_{a}$, respectively [9]. $d N_{t o t, s u}$ is defined as:

$$
d N_{t o t, s u}=d N_{d, s u}+d N_{a, s u}
$$

where $d N_{d, s u}$ is the change in the number of departing particles per unit cross sectional area at the cross section $d u_{d}$ (i.e., at any $r_{c o l l, s u}$ ) after impacting the surface at a particular $r_{s u}$, and $d N_{a, s u}$ is 
the change in the number of arriving particles per unit cross sectional area at the cross section $d u_{a}$ (i.e., at any $r_{\text {coll,su }}$ ) after leaving the nozzle.

$d N_{d, s u}$ is defined as:

$$
d N_{d, s u}=\left.\frac{\Omega_{d}}{v_{d, s u}} \cdot \frac{P_{d}(r) d r}{2 \pi r d r}\right|_{r=r_{\text {coll }, s u}} d u_{d}
$$

where $P_{d}(r)$ is the probability distribution for the departing jet having the same form as equation (5.5), and the subscript $d$ stands for departing. Using equation (5.5), the probability of finding a particle between $r$ and $r+d r, P_{d}(r) d r$, per unit cross sectional area, for the departing trajectory at $r_{\text {coll,su }}$ is:

$$
\left.\frac{P_{d}(r) d r}{2 \pi r d r}\right|_{r=r_{\text {coll }, s u}}=\frac{1}{\pi}\left(\frac{\beta_{d, s u}}{u_{d}+d_{d, s u}}\right)^{2} e^{-\left(\frac{\beta_{d, s u} \cdot r_{c o l l}, s u}{u_{d}+d_{d, s u}}\right)^{2}}
$$

where $r_{\text {coll,su }}$ is defined by equation (5.16), $\beta_{d, s u}$ is the focus coefficient for the departing trajectory, and $u_{d}+d_{d, s u}$ is the distance for the departing trajectory (where $d_{d, s u}$ is the "standoff distance"), measured along the nozzle axis (Figure 5.2), from an imaginary point source (on the imaginary particle jet side) to a point of collision at $r_{\text {coll,su}}$. It is interesting to note that equation (5.20) is independent of the unit ring thickness $d r$ (Figure 5.2), and is evaluated by only considering the location of a particle at a particular point in the collision zone.

The experimentally obtained $\beta$ in equation (5.5), which will henceforth be referred to as $\beta_{a}$, defines the jet focus for the entire arriving jet, or the shape of the entire arriving jet plume, (Chapter 3) and $d$ defines the nozzle-to-target distance, both of which remain constant, regardless of the variable distance $u_{a}$ or the radial position $r$ of an arriving particle. Hence, for the arriving particle, the ratio of $\beta_{a} / d$ remains the same, regardless of the position $u_{a}$ and thus the particle is always launched from a stationary point source (i.e., the nozzle). The same condition is desired for the departing particle.

For $e_{p s}=1$ and $f=0, \beta_{d, s u}$ in equation (5.20) is equal to $\beta_{a}$, and $d_{d, s u}$ is equal to $d$. This is because the incident $\phi_{s u}$ and rebounding $\psi_{s u}$ angles are equal in this case, for all $r_{s u}$, and the incident jet is perfectly reflected. Thus, in Figure 5.2, the imaginary departing jet plume (defined 
by the broken lines) on the imaginary jet side (left-hand side of the bottom diagram) has the same shape as the arriving jet plume (defined by the solid bold lines) on the real jet side (righthand side of the bottom diagram), when reflected across the axis of symmetry (i.e., the target surface). Hence, departing particles are always launched from an imaginary point source which remains stationary (i.e., in Figure 5.2, on the imaginary jet side, the point at $r=0$ and at a distance $d$, measured from the axis of symmetry).

For $e_{p s} \neq 1$ and $f \neq 0$, the incident $\phi_{s u}$ and rebounding $\psi_{s u}$ angles are not equal for any $r_{s u}$, and thus there is really no one $\beta_{d, s u}$ or $d_{d, s u}$ that can define jet divergence for the entire departing jet because the departing particles are always "launched" from a different imaginary point source (located on the imaginary particle jet side) for each $r_{s u}$, as opposed to a stationary point source, as is the case for the arriving jet, or the departing jet for $e_{p s}=1$ and $f=0$. Thus, it will be assumed that the imaginary point source is located on the nozzle axis (i.e., at $r=0$ ) and $\beta_{d, s u}$ and $d_{d, s u}$ will be obtained so that the ratio $\beta_{d, s u} / d_{d, s u}$ remains relatively constant for all values of $r_{s u}$, in order to simulate a stationary imaginary point source from which all departing particles are launched.

To obtain $\beta_{d, s u}$ for $e_{p s} \neq 1$ and $f \neq 0$, the following procedure is used. Equation (5.5) is converted to its angular form. Using the fact that $r=d \tan (\phi)$ and $P(r) d r=P(\phi) d \phi$, where $\phi$ is the angle between the nozzle axis and a particle trajectory and $P(\phi) d \phi$ is the probability of finding a particle between $\phi$ and $\phi+d \phi$ (Chapter 1$), P(\phi)$ is obtained as:

$$
P(\phi)=2 \beta^{2} \frac{\sin \phi}{\cos ^{3}(\phi)} e^{-(\beta \tan \phi)^{2}}
$$

Equation (5.21) is independent of the "standoff distance" $d$. Using the geometry of Figure 5.2, the departing particle trajectory at an angle of $\psi_{s u}$ from the surface (axis of symmetry) can be extended to the imaginary jet side until it intersects the nozzle axis (indicated by the dotted line in Figure 5.2). The angle between this trajectory and the nozzle axis is $\pi / 2-\psi_{s u} \cdot \pi / 2-\psi_{s u}$ can be defined to be the angle at which the probability distribution of equation (5.21) becomes exceedingly small (e.g., 1\%) or the angle at which the majority (e.g., 99\%) of particles are launched from some point source along the nozzle axis (i.e., the "maximum" divergence angle). Thus, using equation (5.21), $\beta_{d, s u}$ can be solved for by setting the probability of finding a particle between $\phi=0$ and $\phi=\pi / 2-\psi_{s u}$ equal to 0.99 [13]: 


$$
\int_{0}^{\pi / 2-\psi_{s u}} 2 \beta_{d, s u}{ }^{2} \frac{\sin \phi}{\cos ^{3}(\phi)} e^{-\left(\beta_{d, s u} \tan \phi\right)^{2}} d \phi=0.99
$$

The Romberg method was used to evaluate the integral on the left-hand side of equation (5.22) (Section 3.2.2) after which $\beta_{d, s u}$ was adjusted until the right-hand side of equation (5.22) was equal to 0.99 . The results for individual $\beta_{d, s u}$ 's can be found in Table 5.1 for the different cases examined (Figures 5.3-5.10). Since the focus coefficient defines the jet divergence for the entire jet (i.e., for a maximum angular trajectory passing through $r_{\max }$ ), and the $\beta_{d, s u}$ obtained from equation (5.22) models the divergence for the departing jet at an angular trajectory passing through $r_{s u}, d_{d, s u}$ for the departing trajectory can be obtained by assuming that $\beta_{d, s u}$ defines the divergence for the entire jet. To do this, the dotted line on the imaginary jet side in Figure 5.2 is offset by a distance of $r_{\max }-r_{s u}$ along the axis of symmetry (as shown by the dotted arrows) and extended until it intersects the nozzle axis (the solid line), which keeps the same "maximum" divergence angle (i.e., $\pi / 2-\psi_{s u}$ ). Using the geometry of Figure 5.2, $d_{d, s u}$ is obtained as:

$$
d_{d, s u}=r_{\max } \tan \psi_{s u}
$$

In order to solve equation (5.17), $d N_{a, s u}$ must also be obtained. $d N_{a, s u}$ is defined as follows:

$$
d N_{a, s u}=\left.\frac{\Omega_{a}}{v_{a}\left(r_{\text {coll }, s u}\right)} \cdot \frac{P_{a}(r) d r}{2 \pi r d r}\right|_{r=r_{\text {coll }, s u}} d u_{a}
$$

where

$$
\left.\frac{P_{a}(r) d r}{2 \pi r d r}\right|_{r=r_{\text {coll }, s u}}=\frac{1}{\pi}\left(\frac{\beta_{a}}{u_{a}}\right)^{2} e^{-\left(\frac{\beta_{a} \cdot r_{c o l l}, s u}{u_{a}}\right)^{2}}
$$

where $P_{a}(r)$ is the probability distribution for the arriving jet having the same form as equation (5.5), the subscript $a$ stands for arriving, and $r_{\text {coll,su }}$ is defined by equation (5.16). $v_{a}\left(r_{\text {coll,su }}\right)$ is the velocity of an arriving particle on a collision trajectory defined by the angle 
$\phi_{\text {coll }, \text { su }}=\tan ^{-1}\left(r_{\text {coll }, s u} / u_{a}\right) . \quad v_{a}\left(r_{\text {coll,su }}\right)$ can be obtained from equations (5.8) and (5.9) by using $r_{\text {coll,su }}$ in place of $r_{s u}$ and $u_{a}$ in place of $d$ :

$$
v_{a}\left(r_{\text {coll }, \text { su }}\right)=v_{a}(0)\left[1+H_{l} \frac{r_{\text {coll }, \text { su }}}{u_{a}}\right]
$$

Following the reasoning of Ref. [9], the total probability of collision and survival for an arriving and a departing particle at $r_{\text {coll,su}}, P_{\text {coll }}\left(r_{\text {coll,su }}\right)$ and $P_{\text {surv }}\left(r_{\text {coll,su }}\right)$, can be obtained by integrating over all the possible collision zones from both the arriving and departing particle trajectories. Using equation (5.16), the fact that $d u_{a}=\left|d u_{d}\right|$ (from $u_{a}=d-u_{d}$ and since the probability of collision cannot be negative), and $d-u_{d}$ in place of $u_{a}$, equations (5.18), (5.19) and (5.24) can be combined so that equation (5.17) can be obtained as:

$$
\begin{aligned}
& \int_{0}^{P_{\text {coll }}\left(r_{\text {coll }, s u)}\right.} \frac{d p_{\text {coll }}\left(r_{\text {coll }, s u}\right)}{1-p_{\text {coll }}\left(r_{\text {coll }, s u}\right)}=(\sigma) d N_{\text {tot }, s u}=4 \pi r_{p}{ }^{2}\left\{\int_{0}^{z_{s u}} \frac{\Omega_{a} \beta_{d, s u}{ }^{2}}{\pi v_{d, s u}\left(u_{d}+d_{d, s u}\right)^{2}} e^{-\left[\frac{\beta_{d, s u} \cdot\left(r_{s u}+\frac{u_{d}}{\tan \psi_{s u}}\right)}{u_{d}+d_{d, s u}}\right]^{2}} d u_{d}+\right. \\
& \left.\int_{0}^{d} \frac{\Omega_{a} \beta_{a}^{2}}{\pi v_{a}(0)\left[1+H_{l} \frac{\left(r_{s u}+u_{d} / \tan \psi_{s u}\right)}{d-u_{d}}\right]\left(d-u_{d}\right)^{2}} e^{\left[u_{d}\right.} d u_{d}\right\}
\end{aligned}
$$

The integral on the right-hand side of equation (5.27) was evaluated using the same method as for equation (5.22) (i.e., the Romberg method). Equation (5.27) can be integrated to obtain:

$$
P_{\text {surv }}\left(r_{\text {coll }, \text { su }}\right)=1-P_{\text {coll }}\left(r_{\text {coll }, \text { su }}\right)=e^{-(\sigma) N_{\text {tot }, \text { su }}}
$$


where $N_{t o t, s u}$ is the total number of departing and arriving particles per unit collision cross sectional area for any $r_{\text {coll,su, }}$, covering all the possible collision zones, and is obtained from evaluating the two integrals in the parentheses on the right-hand side of equation (5.27).

For an arriving particle, since a collision trajectory defined by the angle $\phi_{\text {coll }, s u}$ is the same as a survival trajectory defined by the angle $\phi_{\text {surv,su }}$, i.e., $\phi_{\text {coll }, s u}=$ $\tan ^{-1}\left(r_{\text {coll }, s u} /\left(d-u_{d}\right)=\phi_{\text {surv }, \text { su }}=\tan ^{-1}\left(r_{\text {surv }, \text { su }} / d\right)\right.$ (Figure 5.2), from equation (5.28), it follows that:

$$
P_{\text {surv }}\left(r_{\text {surv }, \text { su }}\right)=P_{\text {surv }}\left(r_{\text {coll }, \text { su }}\right)
$$

where

$$
r_{\text {surv }, s u}=\left(\frac{d}{d-u_{d}}\right) r_{\text {coll }, s u}
$$

A collision between incident and rebounding trajectories for a specific $r_{s u}$ can, in general,

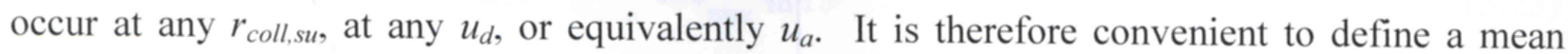
collision distance, $\bar{u}_{d}=z_{s u} / 2$ or $\bar{u}_{a}=d-z_{s u} / 2$, at which a collision "typically" occurs. Substituting this into equation (5.16), the mean $r_{\text {coll,su }}, r_{\text {coll, su }}$, can be obtained as:

$$
r_{\text {coll }, s u}^{-}=r_{s u}+\frac{z_{s u}}{2 \tan \left(\psi_{s u}\right)}
$$

The probability that an arriving particle traveling at the mean collision trajectory defined by the angle $\phi_{\text {coll, su }}^{-}$does not undergo a collision at $r_{\text {coll,su }}^{-}$is also the probability that it survives to the surface at a mean $r_{\text {surv,su}}, r_{\text {surv,su }}^{-}$(Figure 5.2), which, following equation (5.30) and using $\overline{u_{d}}=z_{s u} / 2$, is defined as: 


$$
r_{\text {surv }, \text { su }}^{-}=\left(\frac{d}{d-z_{s u} / 2}\right) r_{\text {coll, su }}^{-}
$$

Thus, to obtain $P_{\text {surv }}\left(r_{\text {surv }, \text { su }}^{-}\right)$, equation (5.31) can be substituted into equation (5.32) along with a user defined $r_{\text {surv,su }}^{-}$. Equation (5.32) can then be solved numerically (see Section 5.3) to obtain an $r_{s u}$ for which equation (5.32) is satisfied. This $r_{s u}$ can then be substituted into all the previous variable quantities in equation (5.27), which can then be solved to obtain equation (5.28) or equivalently equation (5.29). 


\subsection{Percentage of Launched Particles Reaching the Entire Surface}

As was done in Chapter 2, a simple assessment of particle interference effects in erosion testing is the percentage of particles reaching the entire target surface without undergoing prior collisions. This can be obtained by calculating the ratio of the total number of particles surviving to the surface through all the rebound heights (collision cross sections) over which collisions can occur ( $z_{s u}$ 's), $\Omega_{z}$ (particles/s), to the total number of particles launched from the nozzle $\Omega_{a}$ (particles/s), for all values of $r_{s u}$. In Chapter 2, the jet partitioning technique for the nondivergent jet was used, where the jet was divided into rings of $L_{\alpha}$ thickness, where $L_{\alpha}$ was the average space between particles. The total number of particles surviving to the surface $\left(\Omega_{z} / \Omega_{a}\right)$ was obtained by multiplying the probability of survival at each $r$ by the cross sectional area of the $L$ thick ring at that $r$ and then summing up all the results. For the presently considered diverging jet, since the particle spatial distribution (equation (5.5)) already accounts for particle spacing and radial symmetry across the jet, this technique cannot be applied. Instead, $\Omega_{z} / \Omega_{a}$ is determined by evaluating equation (5.27) to obtain equation (5.28) at a mean $r_{s u}, r_{s u}^{-}$. Following equation (5.32), $r_{s u}^{-}$can be obtained by solving the following expression:

$$
\frac{r_{\max }}{2 d}=\tan \left(\frac{\phi_{\max }}{2}\right)=\frac{r_{c o l l}^{-}\left(r_{s u}^{-}\right)}{d-\frac{z\left(\overline{r_{s u}}\right)}{2}}=\frac{r_{s u}^{-}+\frac{z\left(\overline{r_{s u}}\right)}{2 \tan \left(\psi\left(\overline{r_{s u}}\right)\right)}}{d-\frac{z\left(\overline{r_{s u}}\right)}{2}}
$$

where $\phi_{\max } / 2$ is the angle that defines the mean arrival particle trajectory from the nozzle to a mean radial position at the surface, $r_{\max } / 2$, measured from the nozzle axis, and $r_{\text {coll }}^{-}\left(r_{s u}^{-}\right), z\left(r_{s u}^{-}\right)$and $\psi\left(r_{s u}^{-}\right)$are the mean $r_{\text {coll }, s u}$ (equation $\left.(5.31)\right), z_{s u}$ (equation $\left.(5.15)\right)$ and $\psi_{s u}$ (equation (5.14)), respectively, evaluated at a mean $r_{s u}, r_{s u}^{-} \cdot r_{s u}^{-}$is the radial position at the surface that a rebounding particle departs from, traveling on a collision trajectory defined by the angle $\psi\left(\overrightarrow{r_{s u}}\right)$, so that it intersects a mean arrival trajectory at radial position $r_{\text {coll }}^{-}\left(r_{s u}^{-}\right)$and at a 
mean height of $z\left(r_{s u}^{-}\right) / 2$ above the surface. Equation (5.33) is solved for $r_{s u}^{-}$by using the iterative Secant method with the numerical error controlled by a built-in tolerance variable in the Mathcad 11 software (Mathsoft Inc., Cambridge, MA, USA) set at 0.00001. In this method, the Newton method using backward difference approximation is applied using an initial estimate. The method terminates when the two most recent estimates differ by less than the value of the built-in variable TOL $(0.00001) . \quad r_{s u}$ can then be substituted into all the previous variable quantities in equation (5.27), which can then be solved to obtain equation (5.28) or equivalently equation (5.29):

$$
\frac{\Omega_{z}}{\Omega_{a}}=P_{\text {surv }}\left(r_{\text {coll }}^{-}\left(r_{\text {su }}^{-}\right)\right)=P_{\text {surv }}\left(\frac{r_{\max }}{2}\right)
$$

As in Section 2.3, by setting the left-hand side of equation (5.34) to 0.95 , indicating that $95 \%$ of incident particles arrive to the surface without colliding, an estimate of the required maximum critical particle launch frequency (particles/s) for that level of particle interference, $\Omega_{a(c r i t)}$, can be extracted by solving this expression numerically (using the same method that was used to solve equation (5.33) for $r_{s u}^{-}$). This can then be related (see Ref. [12]) to the particle mass flow rate or mass flux:

$$
\dot{M}_{c r i t}=\Omega_{a(c r i t)} m_{p}
$$

where $\dot{M}_{\text {crit }}$ is the critical mass flow rate $(\mathrm{kg} / \mathrm{s})$ and $m_{p}$ is the mass of one particle $(\mathrm{kg})$ :

$$
m_{p}=\frac{4}{3} \pi r_{p}^{3} \cdot \rho_{p}
$$

where $\rho_{p}$ is the particle mass density $\left(\mathrm{kg} / \mathrm{m}^{3}\right)$. Equation (5.35) can be used as a guideline for performing erosion tests free of interference effects. As before, the level of interference that can be tolerated will depend on the application, but 0.95 , used in the work of Ref. [12], seems reasonable. 


\subsection{Results and Discussion}

\subsubsection{Comparison with Computer Simulation of Refs. $[10,12,13]$}

As in Chapter 2, the results of the analytical model can be compared to a previously developed computer simulation which uses particle tracking algorithms to predict interference effects in particle jets $[10,12,13]$, assuming a radially symmetric jet. The modified version of the computer simulation of Ref. [13] was used, so that particles emanating from the nozzle were assumed to follow the Weibull spatial distribution [25], equation (5.5). The program ensures that every particle event is accounted for during execution, even if there are multiple surface or interparticle collisions. For the data collected from the simulation corresponding to the parameters outlined in Table 5.1, a divergent jet, arriving to a rectangular $0.1 \mathrm{~m}$ by $0.1 \mathrm{~m}$ target at normal incidence $\left(\alpha=90^{\circ}\right)$, from an $r_{n}$ just slightly bigger than $r_{p}$ (to simulate a point source), was assumed. The data was collected at steady state (approximately $3-10 \mathrm{~ms}$ of real time, corresponding to when the number of particles entering the system was equal to the number exiting). At maximum, 10,000 particles were in the simulation at a time. The coefficient of restitution for inter-particle collisions was assumed to be unity, since the analytical model does not account for energy loss in particle-to-particle interactions, i.e., it is a single collision model [9].

Using equation (5.34) and the variables outlined in Table 5.1, plots of $\Omega_{z} / \Omega_{a}$ versus $\rho_{s}$ (equation (5.6)) were compared for the modified Anand et al. Weibull model introduced in Section 5.2, and the computer simulation (Figures 5.3-5.10). For all cases but one (Figure 5.5), $\Omega_{z} / \Omega_{a}$ was in the range of 0.713-0.999. Since $\Omega_{a(c r i t)}=0.95$ is included in that range, lower values of $\Omega_{z} / \Omega_{a}$ were not necessary.

For the base case (Figure 5.3), $v_{a}=160 \mathrm{~m} / \mathrm{s}, r_{p}=1.25 \times 10^{-5} \mathrm{~m}, d=0.03 \mathrm{~m}$ and $\beta=15$ were chosen based on work with aluminum oxide in Ref. [25] and Chapter 3 (Figure 3.5). For the present analysis, it was assumed that the angular particles behaved as spheres to obtain a first order approximation to the interference effects. This assumption is supported by the fact that the effect of particle type had a minimal effect on the resulting divergence, i.e., $\beta$ (Section 3.4.1). Only round nozzles were considered in order to facilitate comparisons for the present analytical model, which was derived assuming a diverging jet. To obtain $\rho_{s}$ for each case, only $\Omega_{a}$ was varied so that $\beta$ remains relatively constant since it was found in Chapter 3 (Figure 3.6) that jet 
divergence, for the most part, did not vary significantly with the mass flow rate (i.e., $\Omega_{a}$, equation (5.35)).

For Figure 5.4, a small change in $\beta$, with respect to the base case $\left(v_{a}=160 \mathrm{~m} / \mathrm{s}, \beta=15\right)$, was assumed (since these values were unavailable) for a significant change in velocity (i.e., $v_{a}=$ $100 \mathrm{~m} / \mathrm{s}, \beta=13$ and $\left.v_{a}=200 \mathrm{~m} / \mathrm{s}, \beta=17\right)$. This is justified because for high velocities $\left(v_{a}>100\right.$ $\mathrm{m} / \mathrm{s}$ ), the jet divergence is not expected to vary appreciably with velocity [16]. Based on work in Chapter 3 (Figures 3.11-13), for the aluminum oxide particles, the jet is more focused (i.e., $\beta$ is higher) for higher velocities and less focused (i.e., $\beta$ is lower) for lower velocities.

For Figures 5.5-5.6, $\beta$ 's for different $r_{p}$ values were chosen based on work in Chapter 3 (Figures 3.4-3.6). It was assumed that the velocity remains constant for the different particle sizes in this range.

Finally, for Figure 5.8, $h_{l}$ was calculated with equation (5.9) using $H_{l}=-5$ and $v_{a}=160$ $\mathrm{m} / \mathrm{s}$ ([25], Chapter 4, Figure 4.6) for two different standoff distances. Since the computer simulation assumes a constant velocity distribution across the jet (i.e., $h_{l}=0$ ), only qualitative comparisons can be drawn between results of Figure 5.8 and Figure 5.3 (base case). 
Table 5.1: Variables used for comparison of analytical model with computer simulation.

\begin{tabular}{|c|c|c|c|c|c|c|}
\hline Variable & $\begin{array}{c}\text { Figure } \\
5.3 \text { (Base } \\
\text { Case) }\end{array}$ & $\begin{array}{c}\text { Figure } \\
5.4\end{array}$ & $\begin{array}{c}\text { Figure } \\
5.4\end{array}$ & $\begin{array}{c}\text { Figure } \\
5.5\end{array}$ & $\begin{array}{c}\text { Figure } \\
5.6\end{array}$ & $\begin{array}{c}\text { Figure } \\
5.7\end{array}$ \\
\hline $\begin{array}{c}\Omega_{a}=\Omega_{d} \\
\text { (particles/s) }\end{array}$ & $\begin{array}{l}5 \times 10^{5}- \\
6 \times 10^{6}\end{array}$ & $\begin{array}{l}5 \times 10^{5}- \\
6 \times 10^{6}\end{array}$ & $\begin{array}{l}5 \times 10^{5}- \\
6 \times 10^{6}\end{array}$ & $\begin{array}{l}5 \times 10^{5}- \\
6 \times 10^{6}\end{array}$ & $\begin{array}{l}5 \times 10^{5}- \\
6 \times 10^{6}\end{array}$ & $\begin{array}{l}5 \times 10^{5}- \\
6 \times 10^{6}\end{array}$ \\
\hline$v_{a}(\mathrm{~m} / \mathrm{s})$ & 160 & 100 & 200 & 160 & 160 & 160 \\
\hline$r_{p}(\mathrm{~m})$ & $1.25 \times 10^{-5}$ & $1.25 \times 10^{-5}$ & $1.25 \times 10^{-5}$ & $5 \times 10^{-6}$ & $2.5 \times 10^{-5}$ & $1.25 \times 10^{-5}$ \\
\hline$\beta=\beta_{a}$ & 15 & 13 & 17 & 16 & 14 & 15 \\
\hline$d(\mathrm{~m})$ & 0.03 & 0.03 & 0.03 & 0.03 & 0.03 & 0.015 \\
\hline$h_{l}(\mathrm{~m} / \mathrm{m} \cdot \mathrm{s})$ & 0 & 0 & 0 & 0 & 0 & 0 \\
\hline$e_{p s}$ & 1 & 1 & 1 & 1 & 1 & 1 \\
\hline$f$ & 0 & 0 & 0 & 0 & 0 & 0 \\
\hline$r_{\max }(\mathrm{m})$ & 0.0043 & 0.00496 & 0.00379 & 0.00403 & 0.0046 & 0.00215 \\
\hline$r_{p}^{*}$ & $\begin{array}{c}0.0391- \\
0.469\end{array}$ & $\begin{array}{c}0.0625- \\
0.75\end{array}$ & $\begin{array}{c}0.0313- \\
0.375\end{array}$ & $\begin{array}{c}0.0156- \\
0.188\end{array}$ & $\begin{array}{c}0.0781- \\
0.938\end{array}$ & $\begin{array}{c}0.0391- \\
0.469\end{array}$ \\
\hline$d^{*}$ & $93.8-1130$ & $150-1800$ & $75-900$ & $93.8-1130$ & $93.8-1130$ & $46.9-563$ \\
\hline$\phi_{\max }(\mathrm{rad})$ & 0.142 & 0.164 & 0.126 & 0.134 & 0.152 & 0.142 \\
\hline$\rho_{s}$ & $\begin{array}{l}1.32 \times 10^{-6}- \\
1.58 \times 10^{-5}\end{array}$ & $\begin{array}{l}1.59 \times 10^{-6}- \\
1.91 \times 10^{-5}\end{array}$ & $\begin{array}{l}1.36 \times 10^{-6}- \\
1.63 \times 10^{-5}\end{array}$ & $\begin{array}{l}9.62 \times 10^{-8}- \\
1.15 \times 10^{-6}\end{array}$ & $\begin{array}{l}9.23 \times 10^{-6}- \\
1.11 \times 10^{-4}\end{array}$ & $\begin{array}{l}5.28 \times 10^{-6}- \\
6.34 \times 10^{-5}\end{array}$ \\
\hline$\overline{r_{s u}}(\mathrm{~m})$ & $94 r_{p}$ & $109 r_{p}$ & $84 r_{p}$ & $223 r_{p}$ & $51 r_{p}$ & $47 r_{p}$ \\
\hline$\beta_{d}\left(r_{s u}^{-}\right)$ & N/A & N/A & N/A & N/A & N/A & N/A \\
\hline
\end{tabular}


Table 5.1: ...Continued...

\begin{tabular}{|c|c|c|c|c|c|c|}
\hline Variable & Figure & $\begin{array}{c}\text { Figure } \\
\mathbf{5 . 7}\end{array}$ & $\begin{array}{c}\text { Figure } \\
\mathbf{5 . 8}\end{array}$ & $\begin{array}{c}\text { Figure } \\
\mathbf{5 . 9}\end{array}$ & $\begin{array}{c}\text { Figure } \\
\mathbf{5 . 9}\end{array}$ & $\begin{array}{c}\text { Figure } \\
\mathbf{5 . 9}\end{array}$ \\
\hline$\Omega_{a}=\Omega_{d}$ & $5 \times 10^{5}-$ & $5 \times 10^{5}-$ & $5 \times 10^{5}-$ & $5 \times 10^{5}-$ & $5 \times 10^{5}-$ & $5 \times 10^{5}-$ \\
$(\mathrm{particles} / \mathrm{s})$ & $6 \times 10^{6}$ & $6 \times 10^{6}$ & $6 \times 10^{6}$ & $6 \times 10^{6}$ & $6 \times 10^{6}$ & $6 \times 10^{6}$ \\
\hline$v_{a}(\mathrm{~m} / \mathrm{s})$ & 160 & 160 & 160 & 160 & 160 & 160 \\
\hline$r_{p}(\mathrm{~m})$ & $1.25 \times 10^{-5}$ & $1.25 \times 10^{-5}$ & $1.25 \times 10^{-5}$ & $1.25 \times 10^{-5}$ & $1.25 \times 10^{-5}$ & $1.25 \times 10^{-5}$ \\
\hline$\beta=\beta_{a}$ & 15 & 15 & 15 & 15 & 15 & 15 \\
\hline$d(\mathrm{~m})$ & 0.06 & 0.015 & 0.03 & 0.03 & 0.03 & 0.03 \\
\hline$h_{l}(\mathrm{~m} / \mathrm{m} \cdot \mathrm{s})$ & 0 & $-5.33 \times 10^{4}$ & $-2.67 \times 10^{4}$ & 0 & 0 & 0 \\
\hline$e_{p s}$ & 1 & 1 & 1 & 0.8 & 0.5 & 0.3 \\
\hline$f$ & 0 & 0 & 0 & 0 & 0 & 0 \\
\hline$r_{\max }(\mathrm{m})$ & 0.00859 & 0.00215 & 0.0043 & 0.0043 & 0.0043 & 0.0043 \\
\hline$r_{p}^{*}$ & $0.0391-$ & $0.0391-$ & $0.0391-$ & $0.0391-$ & $0.0391-$ & $0.0391-$ \\
& 0.469 & 0.469 & 0.469 & 0.469 & 0.469 & 0.469 \\
\hline$d^{*}$ & $188-2250$ & $46.9-563$ & $93.8-1130$ & $93.8-1130$ & $93.8-1130$ & $93.8-1130$ \\
\hline$\phi_{\max }$ & 0.142 & 0.142 & 0.142 & 0.142 & 0.142 & 0.142 \\
\hline$\rho_{s}$ & $3.31 \times 10^{-7}-$ & $5.28 \times 10^{-6}-$ & $1.32 \times 10^{-6}-$ & $1.32 \times 10^{-6}-$ & $1.32 \times 10^{-6}-$ & $1.32 \times 10^{-6}-$ \\
& $3.97 \times 10^{-6}$ & $6.34 \times 10^{-5}$ & $1.58 \times 10^{-5}$ & $1.58 \times 10^{-5}$ & $1.58 \times 10^{-5}$ & $1.58 \times 10^{-5}$ \\
\hline$r_{s u}(\mathrm{~m})$ & $185 r_{p}$ & $47 r_{p}$ & $94 r_{p}$ & $92 r_{p}$ & $85 r_{p}$ & $75 r_{p}$ \\
\hline$\beta_{d}\left(r_{s u}^{-}\right)$ & $\mathrm{N} / \mathrm{A}$ & N/A & N/A & 44 & 29 & 20.6 \\
\hline
\end{tabular}


Table 5.1: ...Continued.

\begin{tabular}{|c|c|c|c|c|c|}
\hline Variable & $\begin{array}{c}\text { Figure } \\
\mathbf{5 . 9}\end{array}$ & $\begin{array}{c}\text { Figure } \\
\mathbf{5 . 1 0}\end{array}$ & $\begin{array}{c}\text { Figure } \\
\mathbf{5 . 1 0}\end{array}$ & $\begin{array}{c}\text { Figure } \\
\mathbf{5 . 1 0}\end{array}$ & $\begin{array}{c}\text { Figure } \\
\mathbf{5 . 1 0}\end{array}$ \\
\hline$\Omega_{a}=\Omega_{d}$ & $5 \times 10^{5}-$ & $5 \times 10^{5}-$ & $5 \times 10^{5}-$ & $5 \times 10^{5}-$ & $5 \times 10^{5}-$ \\
$(\mathrm{particles} / \mathrm{s})$ & $6 \times 10^{6}$ & $6 \times 10^{6}$ & $6 \times 10^{6}$ & $6 \times 10^{6}$ & $6 \times 10^{6}$ \\
\hline$v_{a}(\mathrm{~m} / \mathrm{s})$ & 160 & 160 & 160 & 160 & 160 \\
\hline$r_{p}(\mathrm{~m})$ & $1.25 \times 10^{-5}$ & $1.25 \times 10^{-5}$ & $1.25 \times 10^{-5}$ & $1.25 \times 10^{-5}$ & $1.25 \times 10^{-5}$ \\
\hline$\beta=\beta_{a}$ & 15 & 15 & 15 & 15 & 15 \\
\hline$d(\mathrm{~m})$ & 0.03 & 0.03 & 0.03 & 0.03 & 0.03 \\
\hline$h_{l}(\mathrm{~m} / \mathrm{m} \cdot \mathrm{s})$ & 0 & 0 & 0 & 0 & 0 \\
\hline$e_{p s}$ & 0.1 & 1 & 1 & 1 & 1 \\
\hline$f$ & 0 & 0.1 & 0.3 & 0.5 & 0.8 \\
\hline$r_{\max }(\mathrm{m})$ & 0.0043 & 0.0043 & 0.0043 & 0.0043 & 0.0043 \\
\hline$r_{p}{ }^{*}$ & $0.0391-$ & $0.0391-$ & $0.0391-$ & $0.0391-$ & $0.0391-$ \\
& 0.469 & 0.469 & 0.469 & 0.469 & 0.469 \\
\hline$d^{*}$ & $93.8-1130$ & $93.8-1130$ & $93.8-1130$ & $93.8-1130$ & $93.8-1130$ \\
\hline$\phi_{\max }$ & 0.142 & 0.142 & 0.142 & 0.142 & 0.142 \\
\hline$\rho_{s}$ & $1.32 \times 10^{-6}-$ & $1.32 \times 10^{-6}-$ & $1.32 \times 10^{-6}-$ & $1.32 \times 10^{-6}-$ & $1.32 \times 10^{-6}-$ \\
& $1.58 \times 10^{-5}$ & $1.58 \times 10^{-5}$ & $1.58 \times 10^{-5}$ & $1.58 \times 10^{-5}$ & $1.58 \times 10^{-5}$ \\
\hline$r_{s u}(\mathrm{~m})$ & $53 r_{p}$ & $99 r_{p}$ & $99 r_{p}$ & $99 r_{p}$ & $99 r_{p}$ \\
\hline$\beta_{d}\left(r_{s u}{ }^{*}\right)$ & 9.8 & 73 & 73 & 73 & 73 \\
\hline
\end{tabular}

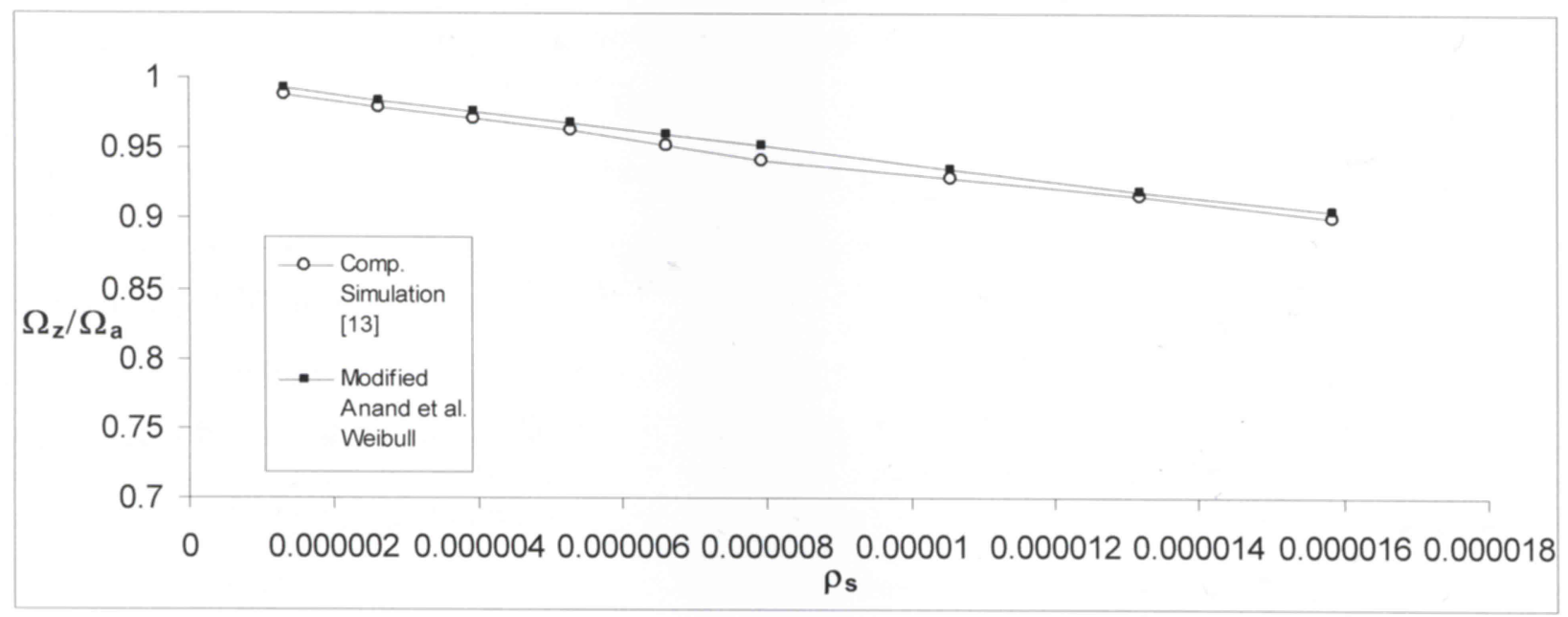

Figure 5.3: Effect of $\rho_{s}$ on $\Omega_{z} / \Omega_{a}$ for the modified Anand et al. Weibull model. Frictionless $(f=0)$ and elastic $\left(e_{p s}=1\right)$ particle-surface collisions assumed along with a constant radial velocity $\left(h_{l}=0\right)$. - $v_{a}=160 \mathrm{~m} / \mathrm{s}, r_{p}=1.25 \times 10^{-5} \mathrm{~m}, \beta=15, d=0.03 \mathrm{~m}$ (Base Case) (Table 5.1). 


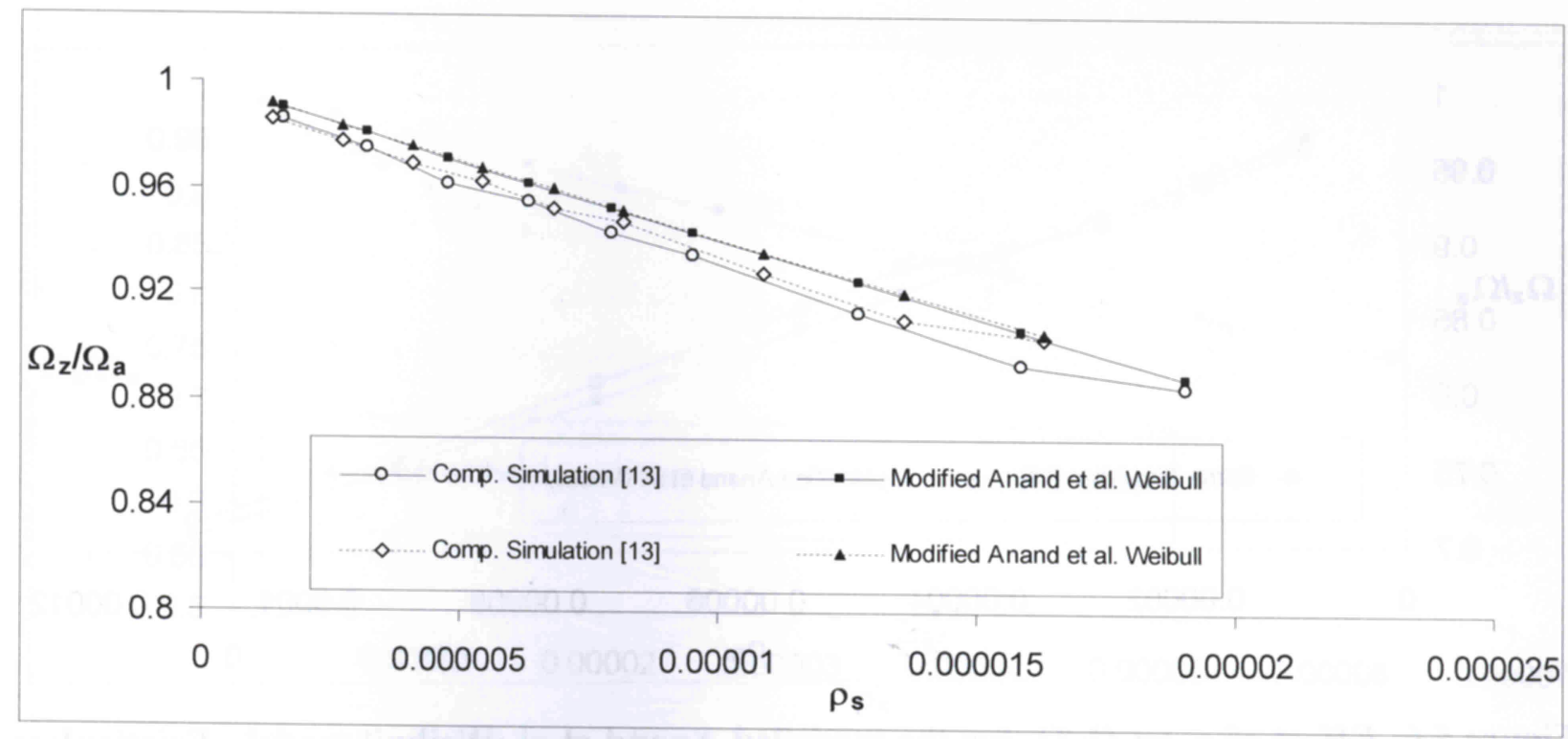

Figure 5.4: Effect of $\rho_{s}$ on $\Omega_{z} / \Omega_{a}$ for the modified Anand et al. Weibull model. Frictionless $(f=0)$ and elastic $\left(e_{p s}=1\right)$ particle-surface collisions assumed along with a constant radial velocity $\left(h_{l}=0\right) . \quad r_{p}=1.25 \times 10^{-5} \mathrm{~m}, d=0.03 \mathrm{~m} .-: v_{a}=100 \mathrm{~m} / \mathrm{s}, \beta=13 ;---: 200 \mathrm{~m} / \mathrm{s}, \beta=17$ (Table 5.1).

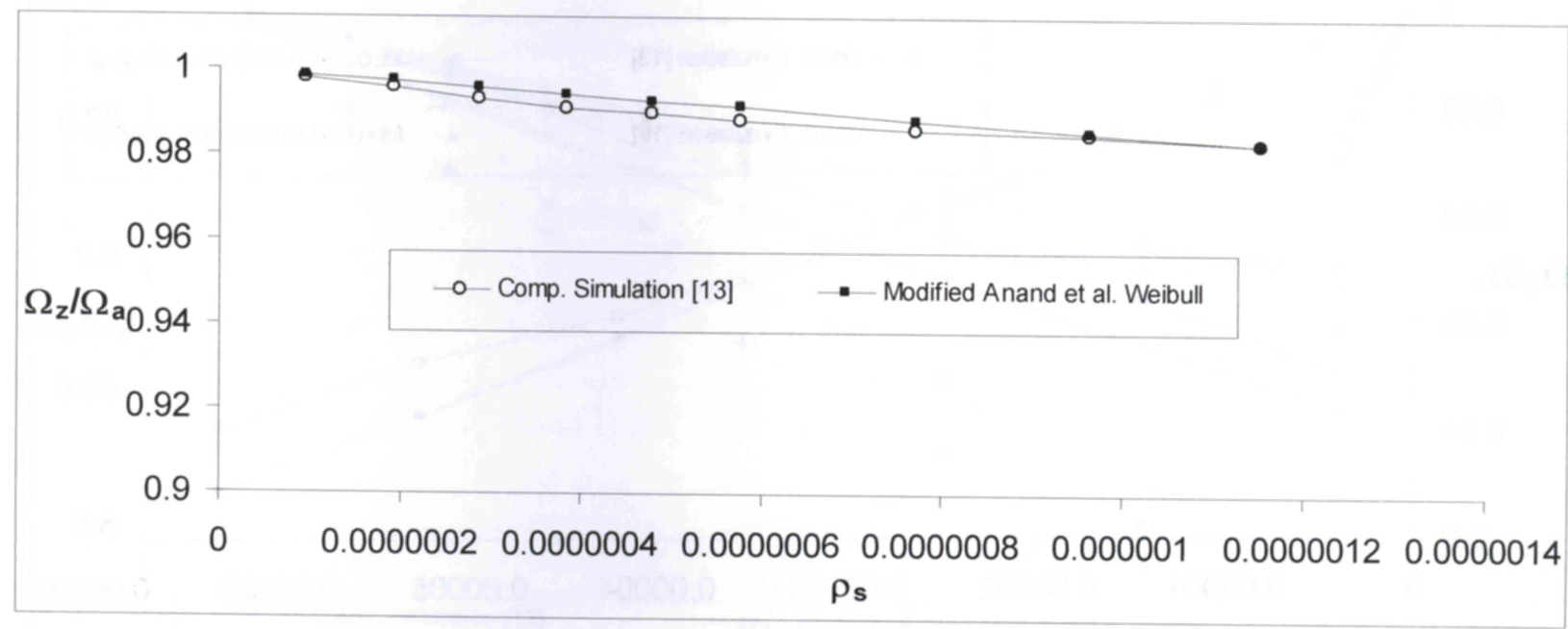

Figure 5.5: Effect of $\rho_{s}$ on $\Omega_{z} / \Omega_{a}$ for the modified Anand et al. Weibull model. Frictionless $(f=0)$ and elastic $\left(e_{p s}=1\right)$ particle-surface collisions assumed along with a constant radial velocity $\left(h_{l}=0\right) . v_{a}=160 \mathrm{~m} / \mathrm{s}, d=0.03 \mathrm{~m} .-: r_{p}=5 \times 10^{-6} \mathrm{~m}, \beta=16$ (Table 5.1). 


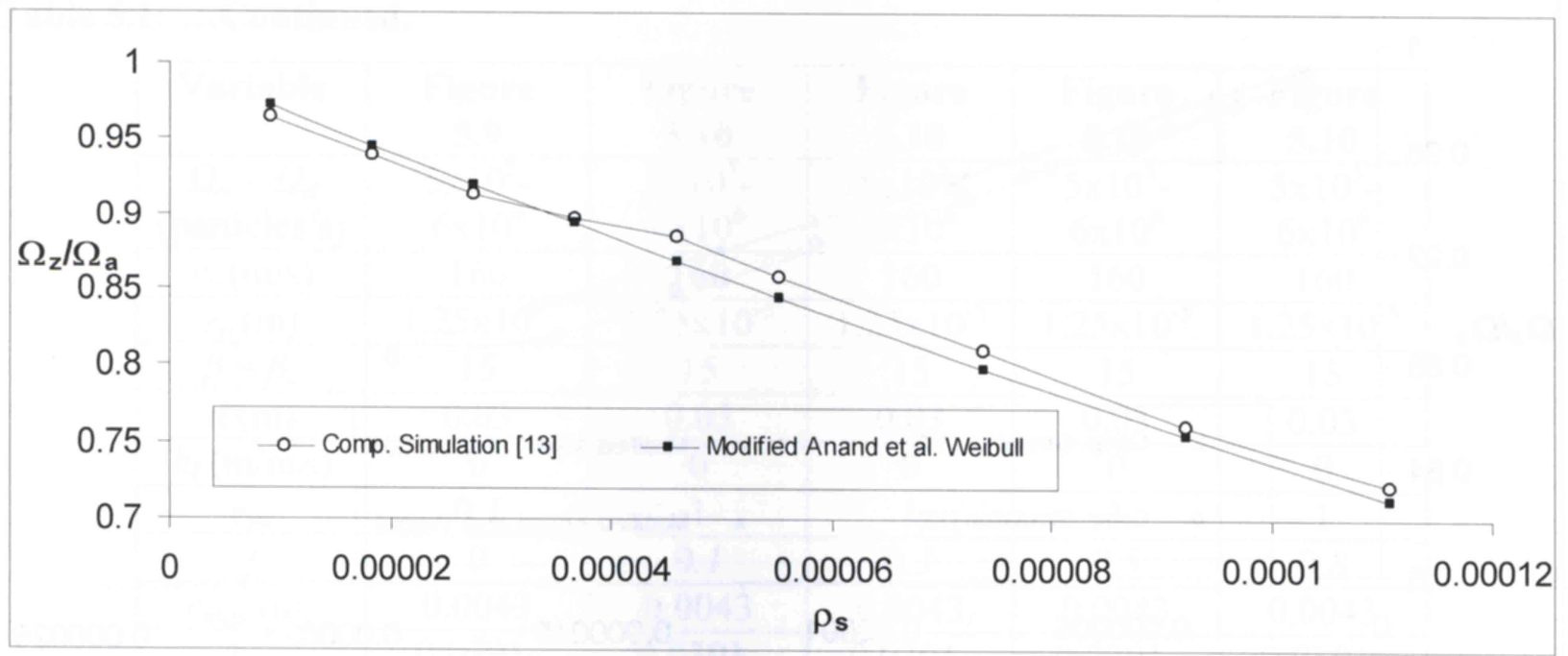

Figure 5.6: Effect of $\rho_{s}$ on $\Omega_{z} / \Omega_{a}$ for the modified Anand et al. Weibull model. Frictionless $(f=0)$ and elastic $\left(e_{p s}=1\right)$ particle-surface collisions assumed along with a constant radial velocity $\left(h_{l}=0\right) . \quad v_{a}=160 \mathrm{~m} / \mathrm{s}, d=0.03 \mathrm{~m} .-: r_{p}=2.5 \times 10^{-5} \mathrm{~m}, \beta=14$ (Table 5.1).

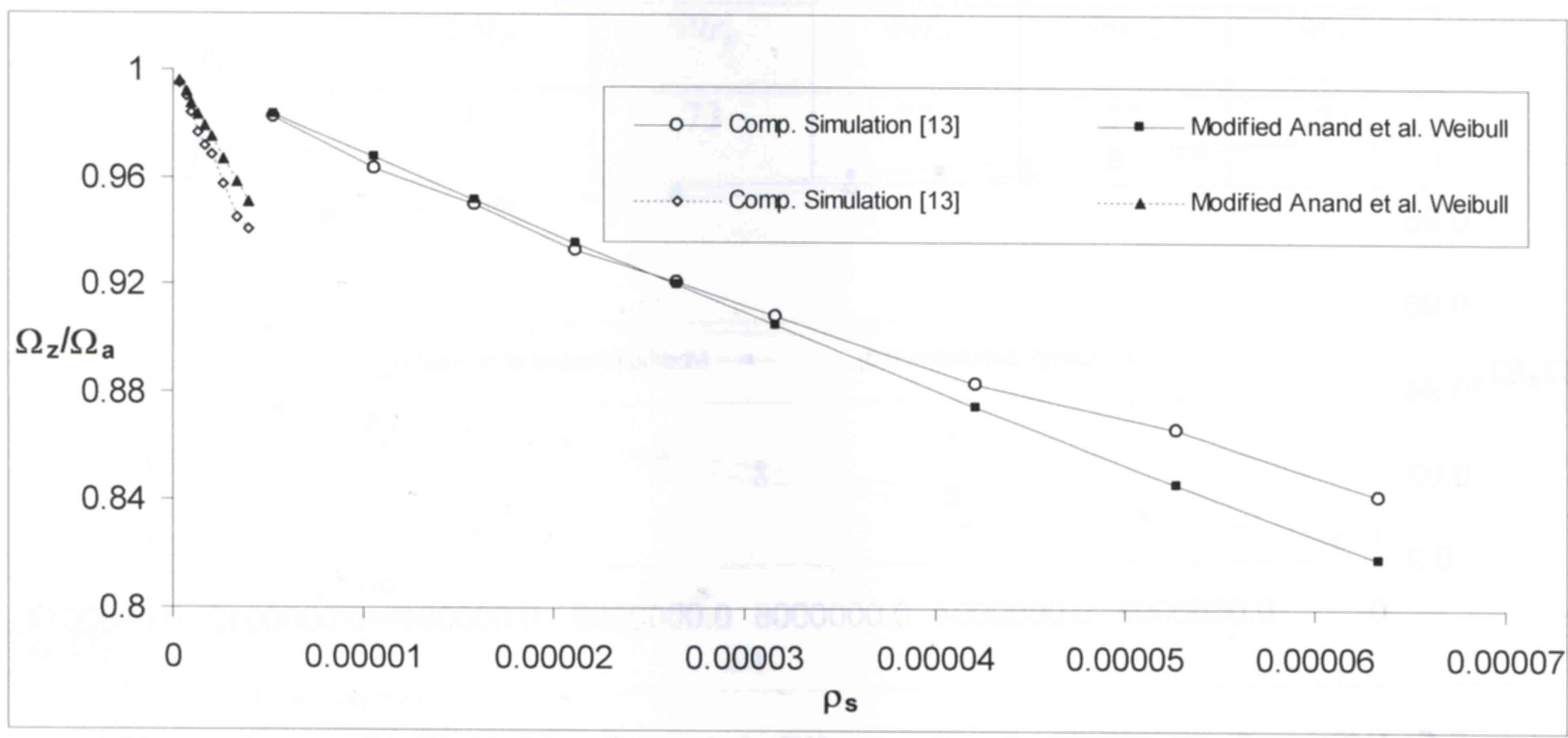

Figure 5.7: Effect of $\rho_{s}$ on $\Omega_{z} / \Omega_{a}$ for the modified Anand et al. Weibull model. Frictionless $(f=0)$ and elastic $\left(e_{p s}=1\right)$ particle-surface collisions assumed along with a constant radial velocity $\left(h_{l}=0\right) . \quad v_{a}=160 \mathrm{~m} / \mathrm{s}, r_{p}=1.25 \times 10^{-5} \mathrm{~m}, \beta=15$. - $: d=0.015 \mathrm{~m} ;----: d=0.06 \mathrm{~m}$ (Table 5.1). 


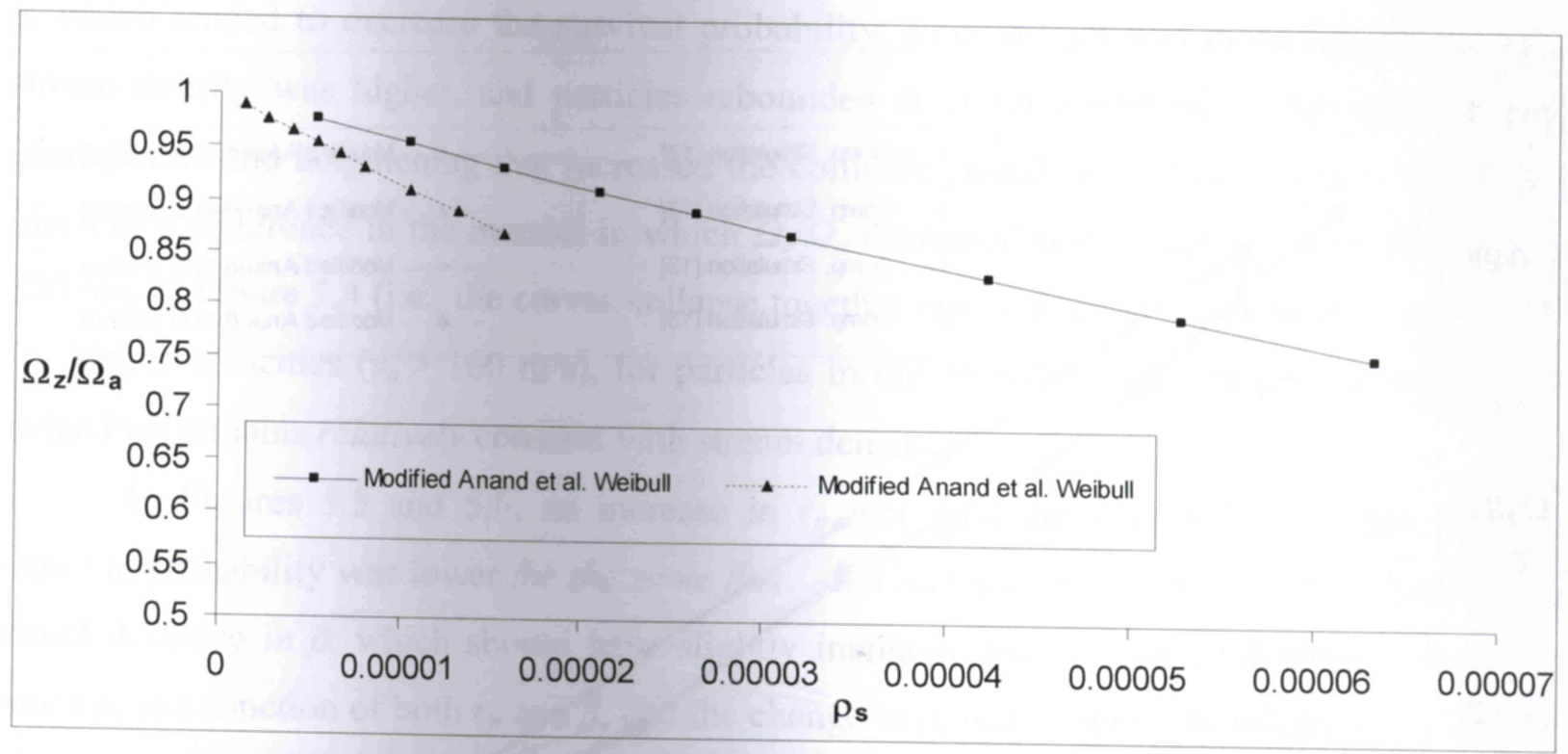

Figure 5.8: Effect of $\rho_{s}$ on $\Omega_{z} / \Omega_{a}$ for the modified Anand et al. Weibull model. Frictionless $(f=0)$ and elastic $\left(e_{p s}=1\right)$ particle-surface collisions assumed. $v_{a}=160 \mathrm{~m} / \mathrm{s}, r_{p}=1.25 \times 10^{-5} \mathrm{~m}$, $\beta=15$. - $: d=0.015 \mathrm{~m}, h_{l}=-5.33 \times 10^{4} \mathrm{~s}^{-1} ;---: d=0.03 \mathrm{~m}, h_{l}=-2.67 \times 10^{4} \mathrm{~s}^{-1}$ (Table 5.1).

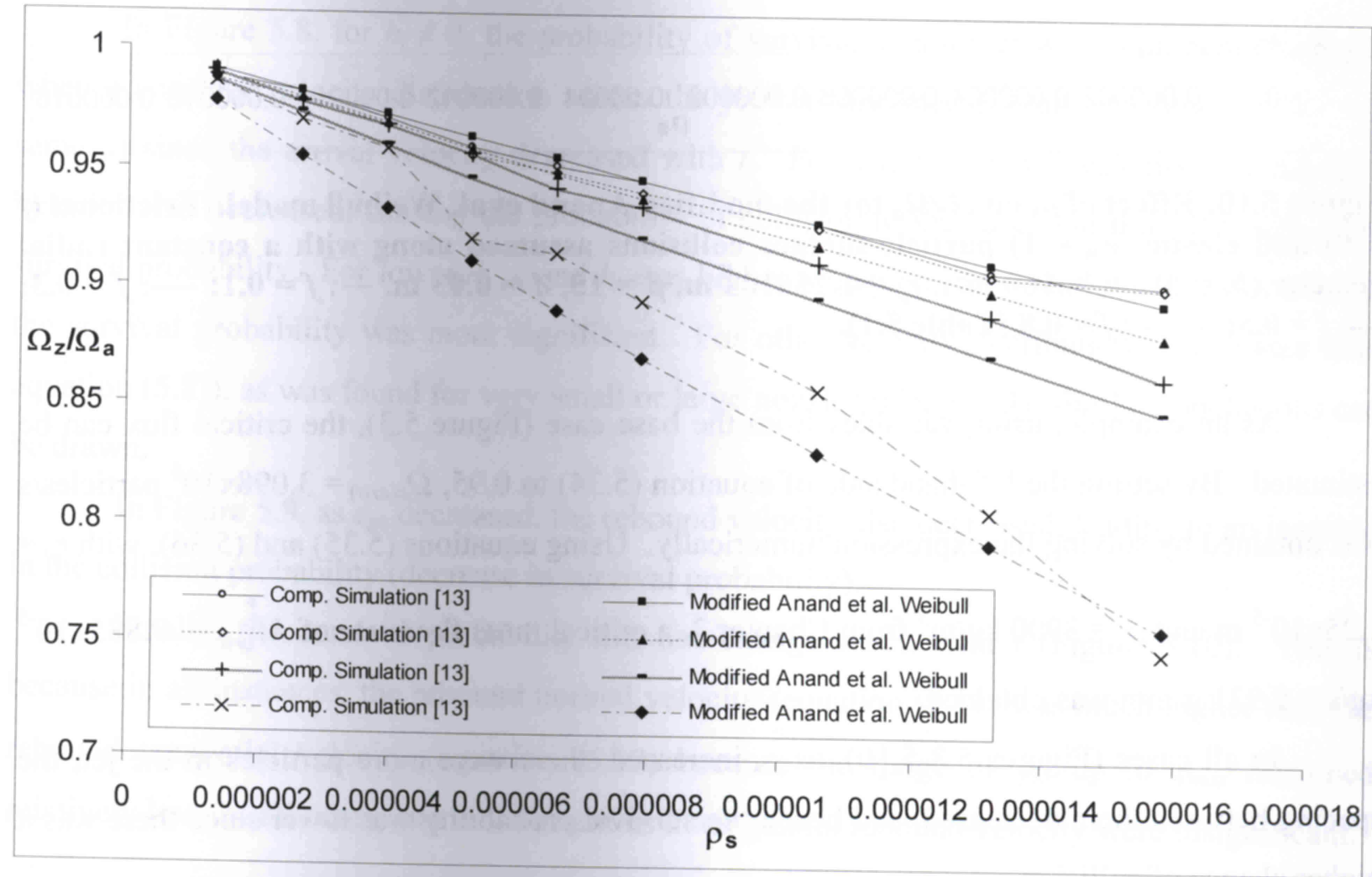

Figure 5.9: Effect of $\rho_{s}$ on $\Omega_{z} / \Omega_{a}$ for the modified Anand et al. Weibull model. Frictionless $(f=0)$ and inelastic $\left(e_{p s} \neq 1\right)$ particle-surface collisions assumed along with a constant radial velocity $\left(h_{l}=0\right) . \quad v_{a}=160 \mathrm{~m} / \mathrm{s}, r_{p}=1.25 \times 10^{-5} \mathrm{~m}, \beta=15, d=0.03 \mathrm{~m}$. - $: e_{p s}=0.8 ;---: e_{p s}=0.5$; .....: $e_{p s}=0.3 ;---: e_{p s}=0.1$ (Table 5.1). 


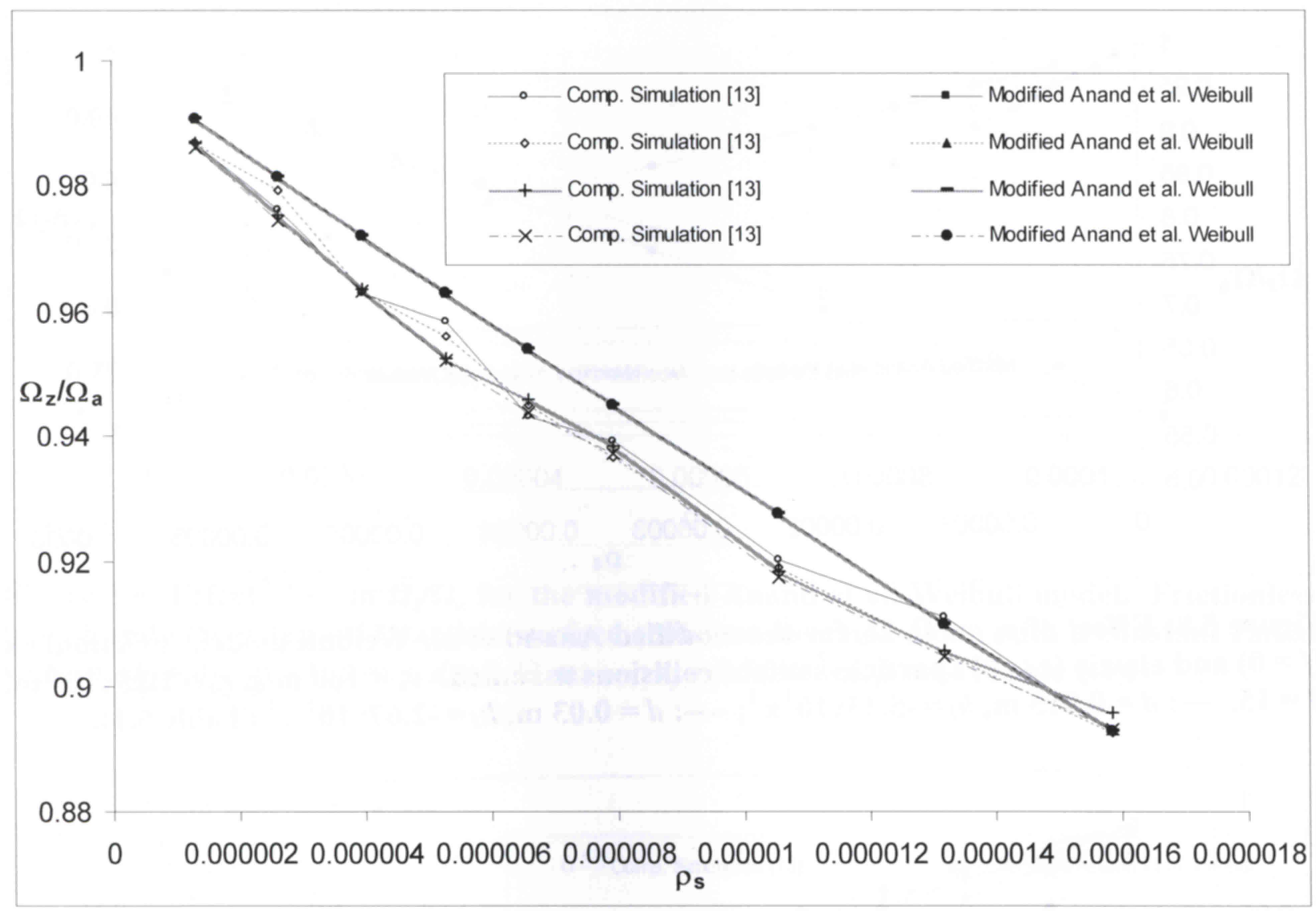

Figure 5.10: Effect of $\rho_{s}$ on $\Omega_{z} / \Omega_{a}$ for the modified Anand et al. Weibull model. Frictional $(f$ $\neq 0)$ and elastic $\left(e_{p s}=1\right)$ particle-surface collisions assumed along with a constant radial velocity $\left(h_{l}=0\right) . \quad v_{a}=160 \mathrm{~m} / \mathrm{s}, r_{p}=1.25 \times 10^{-5} \mathrm{~m}, \beta=15, d=0.03 \mathrm{~m} .-: f=0.1 ;----: f=0.3$; .....: $f=0.5$; - - $: f=0.8$ (Table 5.1).

As an example, using variables from the base case (Figure 5.3), the critical flux can be estimated. By setting the left-hand side of equation (5.34) to $0.95, \Omega_{a(\text { crit })}=3.098 \times 10^{6}$ particles $/ \mathrm{s}$ was obtained by solving the expression numerically. Using equations (5.35) and (5.36), with $r_{p}=$ $1.25 \times 10^{-5} \mathrm{~m}$ and $\rho_{p}=3900 \mathrm{~kg} / \mathrm{m}^{3}$ from Chapter 3 , a critical mass flow rate of $\dot{M}_{\text {crit }}=9.885 \times 10^{-5}$ $\mathrm{kg} / \mathrm{s}=5.931 \mathrm{~g} / \mathrm{min}$ was obtained.

In all cases (Figures 5.3-5.10), as $\rho_{s}$ increased, there were more particles in the jet, the spacing between them decreased, and hence the survival probability was lower since there was a higher chance of collision.

In Figure 5.4, as $v_{a}$ increased, $\rho_{s}$ decreased since the particles within the rebounding and arriving jet traveled at faster velocities, thus decreasing the collision probability (or increasing the survival probability). However, the increase in velocity was accompanied by an increase in 
$\beta$, which tended to decrease the survival probability, since the jet was more focused; i.e., the stream density was higher, and particles rebounded at closer-to-normal angles with a higher average rebound heightening that increased the collision probability. As a result, there was not much of a difference in the manner in which $\Omega_{z} / \Omega_{a}$ decreased with $\rho_{s}$ for $v_{a}=100 \mathrm{~m} / \mathrm{s}$ and $v_{a}=$ $200 \mathrm{~m} / \mathrm{s}$ in Figure 5.4 (i.e., the curves collapse together onto one curve). It can thus be said that for higher velocities $\left(v_{a}>100 \mathrm{~m} / \mathrm{s}\right)$, for particles in the considered size range, the interference behaviour remains relatively constant with stream density $\rho_{s}$.

In Figures 5.5 and 5.6, an increase in $r_{p}$ increased the stream density, and thus the survival probability was lower for the same flux. An increase in $r_{p}$ was also accompanied by a small decrease in $\beta$, which should have slightly increased the survival probability. However, since $\rho_{s}$ is a function of both $r_{p}$ and $\beta$, and the change in $\rho_{s}$ is a lot more significant for variations in $r_{p}$ than for variations in $\beta$, there was an overall increase in $\rho_{s}$ for higher $r_{p}$.

In Figure 5.7, as $d$ increased, the jet volume was higher, which decreased $\rho_{s}$, and thus the probability of survival was higher for the same flux.

In Figure 5.8, for $h_{l} \neq 0$, the probability of survival was lower when compared to cases when a constant velocity distribution across the jet was assumed (Figures 5.3 and 5.7 for the same $d$ ) since the arrival velocity decreased with $r$. For a lower arrival velocity, the rebound velocity also decreased, causing the probability of collision to increase, and thus decreasing the survival probability. For lower $d, \rho_{s}$ and $h_{l}$ was higher (equation (5.9)), and thus the decrease in the survival probability was more significant. For other velocity distributions (i.e., other than equation (5.8)), as was found for very small or large nozzles (Chapter 4), similar conclusions can be drawn.

In Figure 5.9, as $e_{p s}$ decreased, the rebound velocity also decreased, leading to an increase in the collision probability (decrease in survival probability).

Finally, the survival probability did not change much with $f$ (Figure 5.10). This is because in all instances, the rebound normal velocity (equation (5.11) was much higher than the rebound tangential velocity (equation (5.12) because the range of $\phi_{s u}$ up to $\phi_{\max }$ remained relatively low for all cases, and thus variations in tangential rebound velocity were insignificant. 


\subsubsection{Qualitative Comparison with Experimental Observations}

A direct comparison between the present model and experimental results is difficult to make, as there do not appear to be any experimental studies which measure the proportion of particles arriving to the surface without colliding. However, assuming that the erosion rate is proportional to the percentage of particles that arrive to the surface without colliding [9], a qualitative comparison of the results of the model can be made with published experimental findings. As in Chapter 2, experimental results from Refs. [4,5] and [6,7] show that for denser jets (i.e., higher incident particle flux) the erosion rate is lower. The present modified model appears to agree with the experiments, as Figures 5.3-5.10 clearly show that an increase in stream density (i.e., particle flux) resulted in a reduced proportion of particles arriving to the surface without colliding with incident particles. In addition, in Ref. [13], results for scar radius versus the logarithm of mass of erodent used predicted by the computer model, used in obtaining the critical flux at which particle interference becomes significant, matched the results of Ref. [1] with reasonably good agreement. Since the present modified analytical model compares well with the computer model of Ref. [13], it can be concluded that the present model can also be used to accurately obtain the critical flux.

\subsubsection{Advantages over the Computer Simulation, Experimental Methods and Previous Analytical Models}

Despite its limitations, brought upon by the introduction of various assumptions, the model has some advantages over the computer simulation, standard experimental methods, and previous analytical models in obtaining the critical flux. Firstly, the model is readily available, as opposed to the computer simulation. Secondly, it can be used to obtain a quick, fairly accurate estimate of the critical flux compared to the method in Ref. [1], where it might take several hours to perform scar radius measurements to obtain the critical flux, and the computer simulation for higher fluxes, where simulation times can vary between a few minutes to several hours to obtain pertinent data for each specific case used in determining the critical flux. Finally, the present model simulates the more realistic case of diverging jets and can be used for high flux cases, something that cannot be done with previous analytical models of Ref. [8] and [9] and (Chapter 2). 


\section{Chapter 6 Model Limitations}

\subsection{Modified Analytical Models for Non-divergent Jets}

The modified Andrews and Horsfield (Model A) and Anand et al. (Model B) models of Chapter 2 have the following limitations:

1) They are derived for non-divergent jets and assume a uniform velocity across the jet, which is rarely a realistic scenario.

2) They can only be used in cases where the flux is relatively low. At high fluxes, the probabilities of survival rapidly approach 0 .

3) For $\rho_{s} \geq 0.01$, the models begin to deviate from behavior predicted by the computer simulation of Refs. [10,12] (Figures 2.5, 2.6, 2.8 and 2.9). However, the models are still fairly accurate for $\rho_{s} \leq 0.01$. Nevertheless, because these limitations occur at high $\rho_{s}$ or for fluxes well below the "critical" value (e.g., $95 \%$ particle survival) required for erosion testing, above which there are no 'significant' interference effects, they are not serious for the present purposes.

4) They are single collision models; i.e., the arriving and departing particles are allowed to collide only once, after which they are removed from the analysis. Thus, the present models cannot account for multiple collisions (as opposed to the computer simulation of Refs. [10,12]). This can be seen at $\alpha=90^{\circ}$ (Figure 2.7), when the size of the collision zone becomes very large, spanning the standoff distance $d$, where multiple intersections of particle trajectories are possible, and hence the probability of survival is 0 for the present models. In addition, this can be seen for $e_{p s}<0.5$, when multiple collisions near the surface are more frequent, and the present models begin to deviate from the interference behaviour predicted by the computer simulation of Refs. [10,12] (Figure 2.8).

5) As $r_{p}{ }^{*}$ approaches 1, the distance between particle launches approaches zero so particles can overlap as they are being launched (a factor that is accounted for by the computer model). However, none of the analytical models can account for more than one particle 
being launched at a time and thus results for $r_{p}{ }^{*}>0.5$ should be viewed with caution. Nevertheless, the modified Anand et al. model better matches the computer simulation for higher $r_{p}{ }^{*}$, when compared to the modified Andrews and Horsfield model (Figure 2.6). The modified Andrews and Horsfield model is derived at transient state, when the incident and rebound flux are generally not equal, and thus it could work better in modeling the initial transient behavior.

\subsection{Modified Analytical Model for Divergent Jets}

The modified Anand et al. Weibull model of Chapter 5 has the following limitations:

1) The model assumes that particles are launched from a point source rather than a nozzle of finite size, since it incorporates the Weibull distribution into the analysis [25] (Chapter 3). In order to ensure that the point source assumption is valid, a minimum value of $r_{n}$ is desired. Hence, the analysis is only applicable for medium to highly divergent jets and for larger values of $d$ (since $r_{\max }$ is also a function of $d$ (equation (5.7)).

2) It is a single collision model, and thus it cannot account for multiple collisions. Thus, for $e_{p s}<0.5$, when multiple collisions near the surface are more frequent, the present model begins to deviate from the interference behaviour predicted by the modified computer simulation of Ref. [13] (Figure 5.9). 


\section{Chapter 7 Summary and Future Work}

In Chapter 2, two existing analytical models of particle interference in non-divergent particle jets (Refs. $[8,9]$ ) were modified to include radial symmetry of the particle jet across the nozzle axis, inter-particle spacing, and more complex rebound geometry. It was found that the modified Anand et al. model (Model B) best matched the results of an existing computer simulation [10,12], which has previously been compared to experimental results [10]. The analysis showed that increases in stream density (e.g., particle flux) and angle of incidence reduce the percentage of particles that arrive to the target surface without undergoing an interparticle collision. These results qualitatively agree with experimental observations. The modified models allow the critical flux below which inter-particle interference is likely to occur to be determined by solving the analytical expression defined by equation (2.41).

These modified analyses could be improved so that they model collision behavior at $\alpha=$ $90^{\circ}$. In Model B, this can be done by deriving a non-arbitrary average rebound length parameter for the departing jet in the range from $2 r_{n}$ to $d$ [9], which can be used in place of $z_{d \text {,mean }}$ (Section 2.2.2).

In Chapter 3, a novel technique for obtaining the particle spatial distribution across a micro-abrasive jet, by direct particle capture, was devised and rigorously tested. The Weibull distribution [25] matched the experimental results for the majority of cases. For significantly smaller nozzle cross sections, the piecewise Weibull distribution was used. The results were found to be independent of the standoff and flow rate. Also, the jet was found to be more focused for smaller particles, larger nozzles, and higher pressures (except for silica glass beads, where an increase in pressure resulted in a more divergent jet). The effect of particle type was not found to significantly affect divergence.

In Chapter 4, a novel technique for obtaining the velocity distribution across a microabrasive jet, using the results of particle catcher experiments along with eroded profiles obtained from shallow depth micromachining experiments, at various jet conditions, was devised. A linear velocity distribution was found for the majority of cases. For significantly smaller and larger nozzle sizes, a non-linear velocity distribution was found. The results were found to be independent of the standoff, and depended mostly on $M\left(R^{*}\right) / M(0)$ (i.e., $\left.\beta\right)$ and $k$. 
The results of Chapters 2-4 were used to develop a more realistic analytical model of particle interference in Chapter 5 (the modified Anand et al. Weibull model). The existing analytical model of Ref. [9] was modified to include radial symmetry of the particle jet across the nozzle axis, inter-particle spacing, more complex rebound geometry, the effect of a divergent jet, the ability to simulate high flux cases, and the particle spatial (Chapter 3) and velocity (Chapter 4) distributions. The model matched the results of the updated computer simulation of Ref. [13] with good accuracy. As in Chapter 2, the analysis showed that increases in stream density (e.g., particle flux) reduce the percentage of particles that arrive to the target surface without undergoing an inter-particle collision. These results qualitatively agree with experimental observations. The modified model also allows the critical flux below which interparticle interference is likely to occur to be determined by solving the analytical expression defined by equation (5.34).

The model of Chapter 5 could be improved so that it models cases for $\alpha \neq 0$ by using a similar approach as in Chapter 2. In addition, the model could be extended to work for cases where the target surface is curved, as opposed to flat. The final step would be to experimentally verify this model. These are the aims of future work. 


\section{Appendix A Measured $M\left(R^{*}\right) / M(0)$ Values, used in Obtaining the Particle Spatial Distribution across the Jet, along with measured Flow Rates (Corresponding to Section 3.3)}

Table A-1a: Measured $M\left(R^{*}\right) / M(0)$ vs. $r^{*}$ for $10 \mu \mathrm{m}$ angular aluminum oxide blasted at $d=$ $30 \mathrm{~mm}$ for $120 \mathrm{~s}$ at a pressure of $200 \mathrm{kPa}$ and a flow rate of $3.97 \mathrm{~g} / \mathrm{min}$ using $0.76 \mathrm{~mm}$ round nozzle and an $\boldsymbol{r}_{c}$ of $\mathbf{0 . 6} \mathbf{~ m m}$ (corresponding to Figure 3.4).

\begin{tabular}{|c|c|c|c|c|c|c|}
\hline$r(\mathrm{~mm})$ & $r^{*}$ & $M(R)(\mathrm{g})$ & $M\left(R^{*}\right) / M(0)$ & $\begin{array}{c}M(R)(\mathrm{g}) \\
(\text { repeat })\end{array}$ & $\begin{array}{c}M\left(R^{*}\right) / M(0) \\
\text { (repeat) }\end{array}$ & $\begin{array}{c}\text { Standard } \\
\text { Deviation } \\
\left(M\left(R^{*}\right) / M(0)\right)\end{array}$ \\
\hline 0 & 0 & 0.0799 & 1 & 0.081 & 1 & 0 \\
\hline 0.6 & 0.02 & 0.0852 & 1.0663 & 0.0863 & 1.0265 & 0.0199 \\
\hline 1.2 & 0.04 & 0.0622 & 0.7785 & 0.0631 & 0.8005 & 0.01104 \\
\hline 1.8 & 0.06 & 0.042 & 0.5257 & 0.0426 & 0.5365 & 0.00544 \\
\hline 2.4 & 0.08 & 0.0168 & 0.2103 & 0.017 & 0.2265 & 0.00814 \\
\hline 3 & 0.1 & 0.0125 & 0.1564 & 0.0127 & 0.1636 & 0.00356 \\
\hline 3.6 & 0.12 & 0.0052 & 0.06508 & 0.00527 & 0.05645 & 0.00431 \\
\hline 4.2 & 0.14 & 0.0022 & 0.02753 & 0.00223 & 0.02265 & 0.00244 \\
\hline
\end{tabular}

Table A-1b: Measured flow rate for $10 \mu \mathrm{m}$ angular aluminum oxide blasted at $d=30 \mathrm{~mm}$ for $120 \mathrm{~s}$ at a pressure of $200 \mathrm{kPa}$ using $0.76 \mathrm{~mm}$ round nozzle and an $r_{c}$ of $0.6 \mathrm{~mm}$ (corresponding to Figure 3.4).

\begin{tabular}{|c|c|c|c|c|}
\hline $\begin{array}{c}\text { Flow Rate } \\
\text { Measurement } \\
1(\mathrm{~g} / \mathrm{min})\end{array}$ & $\begin{array}{c}\text { Flow Rate } \\
\text { Measurement } \\
2(\mathrm{~g} / \mathrm{min})\end{array}$ & $\begin{array}{c}\text { Flow Rate } \\
\text { Measurement } \\
3(\mathrm{~g} / \mathrm{min})\end{array}$ & $\begin{array}{c}\text { Average Flow } \\
\text { Rate }(\mathrm{g} / \mathrm{min})\end{array}$ & $\begin{array}{c}\text { Standard } \\
\text { Deviation } \\
(\mathrm{g} / \mathrm{min})\end{array}$ \\
\hline 3.95 & 3.91 & 4.06 & 3.97 & 0.06 \\
\hline
\end{tabular}

Table A-2a: Measured $M\left(R^{*}\right) / M(0)$ vs. $r^{*}$ for $25 \mu \mathrm{m}$ angular aluminum oxide blasted at $d=$ $30 \mathrm{~mm}$ for $90 \mathrm{~s}$ at a pressure of $200 \mathrm{kPa}$ and a flow rate of $2.83 \mathrm{~g} / \mathrm{min}$ using $0.76 \mathrm{~mm}$ round nozzle and an $\boldsymbol{r}_{c}$ of $0.23 \mathrm{~mm}$ (corresponding to Figure 3.5).

\begin{tabular}{|c|c|c|c|c|c|c|}
\hline$r(\mathrm{~mm})$ & $r^{*}$ & $M(R)(\mathrm{g})$ & $M\left(R^{*}\right) / M(0)$ & $\begin{array}{c}M(R)(\mathrm{g}) \\
(\text { repeat })\end{array}$ & $\begin{array}{c}M\left(R^{*}\right) / M(0) \\
\text { (repeat) }\end{array}$ & $\begin{array}{c}\text { Standard } \\
\text { Deviation } \\
\left(M\left(R^{*}\right) / M(0)\right)\end{array}$ \\
\hline 0 & 0 & 0.067 & 1 & 0.0691 & 1 & 0 \\
\hline 0.3 & 0.01 & 0.073 & 1.0896 & 0.0744 & 1.0765 & 0.00651 \\
\hline 0.6 & 0.02 & 0.0537 & 0.8015 & 0.0564 & 0.8157 & 0.00709 \\
\hline 1 & 0.03333 & 0.0496 & 0.7403 & 0.0529 & 0.7650 & 0.01233 \\
\hline 1.5 & 0.05 & 0.0385 & 0.5746 & 0.0412 & 0.5965 & 0.01096 \\
\hline 2 & 0.06667 & 0.0257 & 0.3836 & 0.0266 & 0.3857 & 0.00104 \\
\hline 2.5 & 0.08333 & 0.0159 & 0.2373 & 0.0177 & 0.2565 & 0.00962 \\
\hline 3 & 0.1 & 0.0073 & 0.1090 & 0.00709 & 0.1025 & 0.00321 \\
\hline
\end{tabular}


Table A-2b: Measured flow rate for $25 \mu \mathrm{m}$ angular aluminum oxide blasted at $d=\mathbf{3 0} \mathbf{m m}$ for $90 \mathrm{~s}$ at a pressure of $200 \mathrm{kPa}$ using $0.76 \mathrm{~mm}$ round nozzle and an $r_{c}$ of $0.23 \mathrm{~mm}$ (corresponding to Figure 3.5).

\begin{tabular}{|c|c|c|c|c|}
\hline $\begin{array}{c}\text { Flow Rate } \\
\text { Measurement } \\
1(\mathrm{~g} / \mathrm{min})\end{array}$ & $\begin{array}{c}\text { Flow Rate } \\
\text { Measurement } \\
2(\mathrm{~g} / \mathrm{min})\end{array}$ & $\begin{array}{c}\text { Flow Rate } \\
\text { Measurement } \\
3(\mathrm{~g} / \mathrm{min})\end{array}$ & $\begin{array}{c}\text { Average Flow } \\
\text { Rate }(\mathrm{g} / \mathrm{min})\end{array}$ & $\begin{array}{c}\text { Standard } \\
\text { Deviation } \\
(\mathrm{g} / \mathrm{min})\end{array}$ \\
\hline 2.77 & 2.87 & 2.85 & 2.83 & 0.04 \\
\hline
\end{tabular}

Table A-3a: Measured $M\left(R^{*}\right) / M(0)$ vs. $r^{*}$ for $50 \mu \mathrm{m}$ silica glass beads blasted at $d=20 \mathrm{~mm}$ for $120 \mathrm{~s}$ at a pressure of $200 \mathrm{kPa}$ and flow rate of $1.17 \mathrm{~g} / \mathrm{min}$ using $0.76 \mathrm{~mm}$ round nozzle and an $\boldsymbol{r}_{c}$ of $0.23 \mathrm{~mm}$ (corresponding to Figure 3.6).

\begin{tabular}{|c|c|c|c|c|c|c|}
\hline$r(\mathrm{~mm})$ & $r^{*}$ & $M(R)(\mathrm{g})$ & $M\left(R^{*}\right) / M(0)$ & $\begin{array}{c}M(R)(\mathrm{g}) \\
\text { (repeat) }\end{array}$ & $\begin{array}{c}M\left(R^{*}\right) / M(0) \\
\text { (repeat) }\end{array}$ & $\begin{array}{c}\text { Standard } \\
\text { Deviation } \\
\left(M\left(R^{*}\right) / M(0)\right)\end{array}$ \\
\hline 0 & 0 & 0.0711 & 1 & 0.0736 & 1 & 0 \\
\hline 0.3 & 0.015 & 0.0652 & 0.9170 & 0.0655 & 0.8899 & 0.01354 \\
\hline 0.6 & 0.03 & 0.0554 & 0.7792 & 0.0552 & 0.75 & 0.01459 \\
\hline 1 & 0.05 & 0.03 & 0.4219 & 0.031 & 0.4212 & 0.0003726 \\
\hline 1.5 & 0.075 & 0.0174 & 0.2447 & 0.018 & 0.2446 & 0.00008026 \\
\hline 2 & 0.1 & 0.0102 & 0.1435 & 0.011 & 0.1495 & 0.002998 \\
\hline 2.5 & 0.125 & 0.0051 & 0.07173 & 0.0057 & 0.07745 & 0.002858 \\
\hline 3 & 0.15 & 0.0023 & 0.03235 & 0.0029 & 0.03940 & 0.003527 \\
\hline
\end{tabular}

Table A-3b: Measured flow rate for $50 \mu \mathrm{m}$ silica glass beads blasted at $d=20 \mathrm{~mm}$ for $120 \mathrm{~s}$ at a pressure of $200 \mathrm{kPa}$ using $0.76 \mathrm{~mm}$ round nozzle and an $r_{c}$ of $0.23 \mathrm{~mm}$ (corresponding to Figure 3.6).

\begin{tabular}{|c|c|c|c|c|}
\hline $\begin{array}{c}\text { Flow Rate } \\
\text { Measurement } \\
1(\mathrm{~g} / \mathrm{min})\end{array}$ & $\begin{array}{c}\text { Flow Rate } \\
\text { Measurement } \\
2(\mathrm{~g} / \mathrm{min})\end{array}$ & $\begin{array}{c}\text { Flow Rate } \\
\text { Measurement } \\
3(\mathrm{~g} / \mathrm{min})\end{array}$ & $\begin{array}{c}\text { Average Flow } \\
\text { Rate }(\mathrm{g} / \mathrm{min})\end{array}$ & $\begin{array}{c}\text { Standard } \\
\text { Deviation } \\
(\mathrm{g} / \mathrm{min})\end{array}$ \\
\hline 1.14 & 1.20 & 1.18 & 1.17 & 0.02 \\
\hline
\end{tabular}


Table A-4a: Measured $M\left(R^{*}\right) / M(0)$ vs. $r^{*}$ for $50 \mu \mathrm{m}$ silica glass beads blasted at $d=\mathbf{3 0} \mathrm{mm}$ for $120 \mathrm{~s}$ at a pressure of $200 \mathrm{kPa}$ and flow rate of $1.17 \mathrm{~g} / \mathrm{min}$ using $0.76 \mathrm{~mm}$ round nozzle and an $\boldsymbol{r}_{c}$ of $0.23 \mathrm{~mm}$ (corresponding to Figure 3.6).

\begin{tabular}{|c|c|c|c|c|c|c|}
\hline$r(\mathrm{~mm})$ & $r^{*}$ & $M(R)(\mathrm{g})$ & $M\left(R^{*}\right) / M(0)$ & $\begin{array}{c}M(R)(\mathrm{g}) \\
(\text { repeat })\end{array}$ & $\begin{array}{c}M\left(R^{*}\right) / M(0) \\
\text { (repeat) }\end{array}$ & $\begin{array}{c}\text { Standard } \\
\text { Deviation } \\
\left(M\left(R^{*}\right) / M(0)\right)\end{array}$ \\
\hline 0 & 0 & 0.042 & 1 & 0.044 & 1 & 0 \\
\hline 0.3 & 0.01 & 0.0389 & 0.9262 & 0.0411 & 0.9341 & 0.003950 \\
\hline 0.6 & 0.02 & 0.0356 & 0.8476 & 0.0362 & 0.8227 & 0.01245 \\
\hline 1 & 0.0333 & 0.0285 & 0.6786 & 0.0286 & 0.65 & 0.01429 \\
\hline 1.5 & 0.05 & 0.0226 & 0.5381 & 0.0195 & 0.4432 & 0.04746 \\
\hline 2 & 0.0667 & 0.0175 & 0.4167 & 0.0171 & 0.3886 & 0.01402 \\
\hline 2.5 & 0.0833 & 0.0113 & 0.2690 & 0.0104 & 0.2364 & 0.01634 \\
\hline 3 & 0.1 & 0.0099 & 0.2357 & 0.0079 & 0.1795 & 0.02808 \\
\hline
\end{tabular}

Table A-4b: Measured flow rate for $50 \mu \mathrm{m}$ silica glass beads blasted at $d=\mathbf{3 0} \mathrm{mm}$ for $120 \mathrm{~s}$ at a pressure of $200 \mathrm{kPa}$ using $0.76 \mathrm{~mm}$ round nozzle and an $\boldsymbol{r}_{c}$ of $0.23 \mathrm{~mm}$ (corresponding to Figure 3.6).

\begin{tabular}{|c|c|c|c|c|}
\hline $\begin{array}{c}\text { Flow Rate } \\
\text { Measurement } \\
1(\mathrm{~g} / \mathrm{min})\end{array}$ & $\begin{array}{c}\text { Flow Rate } \\
\text { Measurement } \\
2(\mathrm{~g} / \mathrm{min})\end{array}$ & $\begin{array}{c}\text { Flow Rate } \\
\text { Measurement } \\
3(\mathrm{~g} / \mathrm{min})\end{array}$ & $\begin{array}{c}\text { Average Flow } \\
\text { Rate }(\mathrm{g} / \mathrm{min})\end{array}$ & $\begin{array}{c}\text { Standard } \\
\text { Deviation } \\
(\mathrm{g} / \mathrm{min})\end{array}$ \\
\hline 1.14 & 1.20 & 1.18 & 1.17 & 0.02 \\
\hline
\end{tabular}

Table A-5a: Measured $M\left(R^{*}\right) / M(0)$ vs. $r^{*}$ for $50 \mu \mathrm{m}$ silica glass beads blasted at $d=20 \mathrm{~mm}$ for $120 \mathrm{~s}$ at a pressure of $200 \mathrm{kPa}$ and flow rate of $0.59 \mathrm{~g} / \mathrm{min}$ using $0.76 \mathrm{~mm}$ round nozzle and an $\boldsymbol{r}_{c}$ of $0.23 \mathrm{~mm}$ (corresponding to Figure 3.6).

\begin{tabular}{|c|c|c|c|c|c|c|}
\hline$r(\mathrm{~mm})$ & $r^{*}$ & $M(R)(\mathrm{g})$ & $M\left(R^{*}\right) / M(0)$ & $\begin{array}{c}M(R)(\mathrm{g}) \\
\text { (repeat) }\end{array}$ & $\begin{array}{c}M\left(R^{*}\right) / M(0) \\
\text { (repeat) }\end{array}$ & $\begin{array}{c}\text { Standard } \\
\text { Deviation } \\
\left(M\left(R^{*}\right) / M(0)\right)\end{array}$ \\
\hline 0 & 0 & 0.056 & 1 & 0.0571 & 1 & 0 \\
\hline 0.3 & 0.015 & 0.0492 & 0.8786 & 0.0489 & 0.8564 & 0.01109 \\
\hline 0.6 & 0.03 & 0.0438 & 0.7821 & 0.0425 & 0.7443 & 0.01892 \\
\hline 1 & 0.05 & 0.0233 & 0.4161 & 0.0245 & 0.4291 & 0.006500 \\
\hline 1.5 & 0.075 & 0.0156 & 0.2786 & 0.0173 & 0.3030 & 0.01220 \\
\hline 2 & 0.1 & 0.0078 & 0.1393 & 0.0089 & 0.1559 & 0.008291 \\
\hline 2.5 & 0.125 & 0.0035 & 0.0625 & 0.0049 & 0.08581 & 0.01166 \\
\hline 3 & 0.15 & 0.0008 & 0.01429 & 0.0019 & 0.03327 & 0.009495 \\
\hline
\end{tabular}


Table A-5b: Measured flow rate for $50 \mu \mathrm{m}$ silica glass beads blasted at $d=20 \mathrm{~mm}$ for $120 \mathrm{~s}$ at a pressure of $200 \mathrm{kPa}$ using $0.76 \mathrm{~mm}$ round nozzle and an $r_{c}$ of $0.23 \mathrm{~mm}$ (corresponding to Figure 3.6).

\begin{tabular}{|c|c|c|c|c|}
$\begin{array}{c}\text { Flow Rate } \\
\text { Measurement } \\
1(\mathrm{~g} / \mathrm{min})\end{array}$ & $\begin{array}{c}\text { Flow Rate } \\
\text { Measurement } \\
2(\mathrm{~g} / \mathrm{min})\end{array}$ & $\begin{array}{c}\text { Flow Rate } \\
\text { Measurement } \\
3(\mathrm{~g} / \mathrm{min})\end{array}$ & $\begin{array}{c}\text { Average Flow } \\
\text { Rate }(\mathrm{g} / \mathrm{min})\end{array}$ & $\begin{array}{c}\text { Standard } \\
\text { Deviation } \\
(\mathrm{g} / \mathrm{min})\end{array}$ \\
\hline 0.67 & 0.48 & 0.63 & 0.59 & 0.08 \\
\hline
\end{tabular}

Table A-6a: Measured $M\left(R^{*}\right) / M(0)$ vs. $r^{*}$ for $25 \mu \mathrm{m}$ angular aluminum oxide blasted at $d=$ $10 \mathrm{~mm}$ for $180 \mathrm{~s}$ at a pressure of $200 \mathrm{kPa}$ and flow rate of $0.36 \mathrm{~g} / \mathrm{min}$ using $0.46 \mathrm{~mm} \mathrm{H}$.P. round nozzle and an $\boldsymbol{r}_{c}$ of $0.23 \mathrm{~mm}$ (corresponding to Figure 3.7).

\begin{tabular}{|c|c|c|c|c|c|c|}
\hline$r(\mathrm{~mm})$ & $r^{*}$ & $M(R)(\mathrm{g})$ & $M\left(R^{*}\right) / M(0)$ & $\begin{array}{c}M(R)(\mathrm{g}) \\
(\text { repeat })\end{array}$ & $\begin{array}{c}M\left(R^{*}\right) / M(0) \\
(\text { repeat })\end{array}$ & $\begin{array}{c}\text { Standard } \\
\text { Deviation } \\
\left(M\left(R^{*}\right) / M(0)\right)\end{array}$ \\
\hline 0 & 0 & 0.2222 & 1 & 0.228 & 1 & 0 \\
\hline 0.3 & 0.03 & 0.3231 & 1.4541 & 0.3139 & 1.3765 & 0.03878 \\
\hline 0.6 & 0.06 & 0.194 & 0.8731 & 0.2014 & 0.8832 & 0.005064 \\
\hline 0.9 & 0.09 & 0.0859 & 0.3866 & 0.0973 & 0.4265 & 0.01998 \\
\hline 1.2 & 0.12 & 0.0223 & 0.1004 & 0.0311 & 0.1365 & 0.01809 \\
\hline 1.5 & 0.15 & 0.0083 & 0.03735 & 0.0104 & 0.04566 & 0.004151 \\
\hline 1.8 & 0.18 & 0.0031 & 0.01395 & 0.00403 & 0.01765 & 0.001851 \\
\hline 2.1 & 0.21 & 0.0009 & 0.004050 & 0.0018 & 0.007898 & 0.001924 \\
\hline
\end{tabular}

Table A-6b: Measured flow rate for $25 \mu \mathrm{m}$ angular aluminum oxide blasted at $d=10 \mathrm{~mm}$ for $180 \mathrm{~s}$ at a pressure of $200 \mathrm{kPa}$ using $0.46 \mathrm{~mm}$ H.P. round nozzle and an $r_{c}$ of $0.23 \mathrm{~mm}$ (corresponding to Figure 3.7).

\begin{tabular}{|c|c|c|c|c|}
\hline $\begin{array}{c}\text { Flow Rate } \\
\text { Measurement } \\
1(\mathrm{~g} / \mathrm{min})\end{array}$ & $\begin{array}{c}\text { Flow Rate } \\
\text { Measurement } \\
2(\mathrm{~g} / \mathrm{min})\end{array}$ & $\begin{array}{c}\text { Flow Rate } \\
\text { Measurement } \\
3(\mathrm{~g} / \mathrm{min})\end{array}$ & $\begin{array}{c}\text { Average Flow } \\
\text { Rate }(\mathrm{g} / \mathrm{min})\end{array}$ & $\begin{array}{c}\text { Standard } \\
\text { Deviation } \\
(\mathrm{g} / \mathrm{min})\end{array}$ \\
\hline 0.24 & 0.47 & 0.38 & 0.36 & 0.09 \\
\hline
\end{tabular}


Table A-7a: Measured $M\left(R^{*}\right) / M(0)$ vs. $r^{*}$ for $25 \mu \mathrm{m}$ angular aluminum oxide blasted at $d=$ $30 \mathrm{~mm}$ for $60 \mathrm{~s}$ at a pressure of $200 \mathrm{kPa}$ and flow rate of $4.75 \mathrm{~g} / \mathrm{min}$ using $1.5 \mathrm{~mm}$ H.P. round nozzle and an $\boldsymbol{r}_{c}$ of $0.23 \mathrm{~mm}$ (corresponding to Figure 3.8).

\begin{tabular}{|c|c|c|c|c|c|c|}
\hline$r(\mathrm{~mm})$ & $r^{*}$ & $M(R)(\mathrm{g})$ & $M\left(R^{*}\right) / M(0)$ & $\begin{array}{c}M(R)(\mathrm{g}) \\
(\text { repeat })\end{array}$ & $\begin{array}{c}M\left(R^{*}\right) / M(0) \\
\text { (repeat) }\end{array}$ & $\begin{array}{c}\text { Standard } \\
\text { Deviation } \\
\left(M\left(R^{*}\right) / M(0)\right)\end{array}$ \\
\hline 0 & 0 & 0.0728 & 1 & 0.0756 & 1 & 0 \\
\hline 0.5 & 0.0167 & 0.0576 & 0.7912 & 0.0589 & 0.7791 & 0.006054 \\
\hline 1 & 0.0333 & 0.0457 & 0.6277 & 0.0451 & 0.5966 & 0.01559 \\
\hline 1.5 & 0.05 & 0.02 & 0.2747 & 0.025 & 0.3307 & 0.02798 \\
\hline 2 & 0.0667 & 0.0115 & 0.1580 & 0.0134 & 0.1772 & 0.009641 \\
\hline 2.5 & 0.0833 & 0.006 & 0.08242 & 0.0069 & 0.09127 & 0.004426 \\
\hline 3 & 0.1 & 0.0045 & 0.06181 & 0.0057 & 0.07540 & 0.006792 \\
\hline 3.5 & 0.117 & 0.002 & 0.02747 & 0.0024 & 0.03175 & 0.002137 \\
\hline
\end{tabular}

Table A-7b: Measured flow rate for $25 \mu \mathrm{m}$ angular aluminum oxide blasted at $d=30 \mathrm{~mm}$ for $60 \mathrm{~s}$ at a pressure of $200 \mathrm{kPa}$ using $1.5 \mathrm{~mm}$ H.P. round nozzle and an $r_{c}$ of $0.23 \mathrm{~mm}$ (corresponding to Figure 3.8).

\begin{tabular}{|c|c|c|c|c|}
\hline $\begin{array}{c}\text { Flow Rate } \\
\text { Measurement } \\
1(\mathrm{~g} / \mathrm{min})\end{array}$ & $\begin{array}{c}\text { Flow Rate } \\
\text { Measurement } \\
2(\mathrm{~g} / \mathrm{min})\end{array}$ & $\begin{array}{c}\text { Flow Rate } \\
\text { Measurement } \\
3(\mathrm{~g} / \mathrm{min})\end{array}$ & $\begin{array}{c}\text { Average Flow } \\
\text { Rate }(\mathrm{g} / \mathrm{min})\end{array}$ & $\begin{array}{c}\text { Standard } \\
\text { Deviation } \\
(\mathrm{g} / \mathrm{min})\end{array}$ \\
\hline 4.95 & 4.83 & 4.48 & 4.75 & 0.20 \\
\hline
\end{tabular}

Table A-8a: Measured $M\left(R^{*}\right) / M(0)$ vs. $r^{*}$ for $50 \mu \mathrm{m}$ silica glass beads blasted at $d=10 \mathrm{~mm}$ for $180 \mathrm{~s}$ at a pressure of $200 \mathrm{kPa}$ and flow rate of $0.23 \mathrm{~g} / \mathrm{min}$ using $0.46 \mathrm{~mm}$ H.P. round nozzle and an $\boldsymbol{r}_{c}$ of $0.23 \mathrm{~mm}$ (corresponding to Figure 3.9).

\begin{tabular}{|c|c|c|c|c|c|c|}
\hline$r(\mathrm{~mm})$ & $r^{*}$ & $M(R)(\mathrm{g})$ & $M\left(R^{*}\right) / M(0)$ & $\begin{array}{c}M(R)(\mathrm{g}) \\
(\text { repeat })\end{array}$ & $\begin{array}{c}M\left(R^{*}\right) / M(0) \\
\text { (repeat) }\end{array}$ & $\begin{array}{c}\text { Standard } \\
\text { Deviation } \\
\left(M\left(R^{*}\right) / M(0)\right)\end{array}$ \\
\hline 0 & 0 & 0.1072 & 1 & 0.1093 & 1 & 0 \\
\hline 0.3 & 0.03 & 0.1268 & 1.1828 & 0.1202 & 1.09988 & 0.04148 \\
\hline 0.6 & 0.06 & 0.1064 & 0.9925 & 0.1023 & 0.9360 & 0.02829 \\
\hline 0.9 & 0.09 & 0.0706 & 0.6586 & 0.0674 & 0.6167 & 0.02095 \\
\hline 1.2 & 0.12 & 0.031 & 0.2892 & 0.0325 & 0.2976 & 0.004195 \\
\hline 1.5 & 0.15 & 0.021 & 0.1959 & 0.0183 & 0.1679 & 0.01402 \\
\hline 1.8 & 0.18 & 0.015 & 0.1399 & 0.0116 & 0.1057 & 0.01713 \\
\hline 2.1 & 0.21 & 0.01 & 0.09328 & 0.00739 & 0.06758 & 0.01285 \\
\hline
\end{tabular}


Table A-8b: Measured flow rate for $50 \mu \mathrm{m}$ silica glass beads blasted at $d=10 \mathrm{~mm}$ for $180 \mathrm{~s}$ at a pressure of $200 \mathrm{kPa}$ using $0.46 \mathrm{~mm} \mathrm{H.P.} \mathrm{round} \mathrm{nozzle} \mathrm{and} \mathrm{an} r_{c}$ of $0.23 \mathrm{~mm}$ (corresponding to Figure 3.9).

\begin{tabular}{|c|c|c|c|c|}
\hline $\begin{array}{c}\text { Flow Rate } \\
\text { Measurement } \\
1(\mathrm{~g} / \mathrm{min})\end{array}$ & $\begin{array}{c}\text { Flow Rate } \\
\text { Measurement } \\
2(\mathrm{~g} / \mathrm{min})\end{array}$ & $\begin{array}{c}\text { Flow Rate } \\
\text { Measurement } \\
3(\mathrm{~g} / \mathrm{min})\end{array}$ & $\begin{array}{c}\text { Average Flow } \\
\text { Rate }(\mathrm{g} / \mathrm{min})\end{array}$ & $\begin{array}{c}\text { Standard } \\
\text { Deviation } \\
(\mathrm{g} / \mathrm{min})\end{array}$ \\
\hline 0.18 & 0.28 & 0.24 & 0.23 & 0.04 \\
\hline
\end{tabular}

Table A-9a: Measured $M\left(R^{*}\right) / M(0)$ vs. $r^{*}$ for $50 \mu \mathrm{m}$ silica glass beads blasted at $d=20 \mathrm{~mm}$ for $240 \mathrm{~s}$ at a pressure of $200 \mathrm{kPa}$ and flow rate of $0.23 \mathrm{~g} / \mathrm{min}$ using $0.46 \mathrm{~mm}$ H.P. round nozzle and an $\boldsymbol{r}_{c}$ of $0.23 \mathrm{~mm}$ (corresponding to Figure 3.9).

\begin{tabular}{|c|c|c|c|c|c|c|}
\hline$r(\mathrm{~mm})$ & $r^{*}$ & $M(R)(\mathrm{g})$ & $M\left(R^{*}\right) / M(0)$ & $\begin{array}{c}M(R)(\mathrm{g}) \\
(\text { repeat })\end{array}$ & $\begin{array}{c}M\left(R^{*}\right) / M(0) \\
(\text { repeat })\end{array}$ & $\begin{array}{c}\text { Standard } \\
\text { Deviation } \\
\left(M\left(R^{*}\right) / M(0)\right)\end{array}$ \\
\hline 0 & 0 & 0.0395 & 1 & 0.0398 & 1 & 0 \\
\hline 0.3 & 0.015 & 0.0474 & 1.2 & 0.0478 & 1.2 & 0 \\
\hline 0.6 & 0.03 & 0.0544 & 1.3772 & 0.0520 & 1.3059 & 0.03568 \\
\hline 1 & 0.05 & 0.0442 & 1.1190 & 0.0436 & 1.0957 & 0.01166 \\
\hline 1.5 & 0.075 & 0.0237 & 0.6 & 0.0243 & 0.6101 & 0.005028 \\
\hline 2 & 0.1 & 0.0129 & 0.3266 & 0.0139 & 0.3490 & 0.0112 \\
\hline 2.5 & 0.125 & 0.0098 & 0.2481 & 0.0102 & 0.2567 & 0.004279 \\
\hline 3 & 0.15 & 0.006 & 0.1519 & 0.0070 & 0.1765 & 0.01228 \\
\hline
\end{tabular}

Table A-9b: Measured flow rate for $50 \mu \mathrm{m}$ silica glass beads blasted at $d=20 \mathrm{~mm}$ for $240 \mathrm{~s}$ at a pressure of $200 \mathrm{kPa}$ using $0.46 \mathrm{~mm}$ H.P. round nozzle and an $r_{c}$ of $0.23 \mathrm{~mm}$ (corresponding to Figure 3.9).

\begin{tabular}{|c|c|c|c|c|}
\hline $\begin{array}{c}\text { Flow Rate } \\
\text { Measurement } \\
1(\mathrm{~g} / \mathrm{min})\end{array}$ & $\begin{array}{c}\text { Flow Rate } \\
\text { Measurement } \\
2(\mathrm{~g} / \mathrm{min})\end{array}$ & $\begin{array}{c}\text { Flow Rate } \\
\text { Measurement } \\
3(\mathrm{~g} / \mathrm{min})\end{array}$ & $\begin{array}{c}\text { Average Flow } \\
\text { Rate }(\mathrm{g} / \mathrm{min})\end{array}$ & $\begin{array}{c}\text { Standard } \\
\text { Deviation } \\
(\mathrm{g} / \mathrm{min})\end{array}$ \\
\hline 0.18 & 0.28 & 0.24 & 0.23 & 0.04 \\
\hline
\end{tabular}


Table A-10a: Measured $M\left(R^{*}\right) / M(0)$ vs. $r^{*}$ for $50 \mu \mathrm{m}$ silica glass beads blasted at $d=20 \mathrm{~mm}$ for $60 \mathrm{~s}$ at a pressure of $200 \mathrm{kPa}$ and flow rate of $1.31 \mathrm{~g} / \mathrm{min}$ using $1.5 \mathrm{~mm} \mathrm{H} . \mathrm{P}$. round nozzle and an $\boldsymbol{r}_{c}$ of $0.23 \mathrm{~mm}$ (corresponding to Figure 3.10).

\begin{tabular}{|c|c|c|c|c|c|c|}
\hline$r(\mathrm{~mm})$ & $r^{*}$ & $M(R)(\mathrm{g})$ & $M\left(R^{*}\right) / M(0)$ & $\begin{array}{c}M(R)(\mathrm{g}) \\
\text { (repeat) }\end{array}$ & $\begin{array}{c}M\left(R^{*}\right) / M(0) \\
\text { (repeat) }\end{array}$ & $\begin{array}{c}\text { Standard } \\
\text { Deviation } \\
\left(M\left(R^{*}\right) / M(0)\right)\end{array}$ \\
\hline 0 & 0 & 0.0843 & 1 & 0.088 & 1 & 0 \\
\hline 0.3 & 0.015 & 0.0747 & 0.8861 & 0.0764 & 0.8682 & 0.00897 \\
\hline 0.6 & 0.03 & 0.0623 & 0.7390 & 0.0642 & 0.7295 & 0.004741 \\
\hline 1 & 0.05 & 0.0313 & 0.3712 & 0.0341 & 0.3875 & 0.008103 \\
\hline 1.5 & 0.075 & 0.0168 & 0.1993 & 0.0175 & 0.1989 & 0.0002123 \\
\hline 2 & 0.1 & 0.0055 & 0.06524 & 0.0065 & 0.07386 & 0.00431 \\
\hline 2.5 & 0.125 & 0.0055 & 0.06524 & 0.0051 & 0.05795 & 0.003644 \\
\hline 3 & 0.15 & 0.0018 & 0.02135 & 0.0025 & 0.02841 & 0.003528 \\
\hline
\end{tabular}

Table A-10b: Measured flow rate for $50 \mu \mathrm{m}$ silica glass beads blasted at $d=20 \mathrm{~mm}$ for $60 \mathrm{~s}$ at a pressure of $200 \mathrm{kPa}$ using $1.5 \mathrm{~mm}$ H.P. round nozzle and an $r_{c}$ of $0.23 \mathrm{~mm}$ (corresponding to Figure 3.10).

\begin{tabular}{|c|c|c|c|c|}
\hline $\begin{array}{c}\text { Flow Rate } \\
\text { Measurement } \\
1(\mathrm{~g} / \mathrm{min})\end{array}$ & $\begin{array}{c}\text { Flow Rate } \\
\text { Measurement } \\
2(\mathrm{~g} / \mathrm{min})\end{array}$ & $\begin{array}{c}\text { Flow Rate } \\
\text { Measurement } \\
3(\mathrm{~g} / \mathrm{min})\end{array}$ & $\begin{array}{c}\text { Average Flow } \\
\text { Rate }(\mathrm{g} / \mathrm{min})\end{array}$ & $\begin{array}{c}\text { Standard } \\
\text { Deviation } \\
(\mathrm{g} / \mathrm{min})\end{array}$ \\
\hline 1.29 & 1.32 & 1.33 & 1.31 & 0.02 \\
\hline
\end{tabular}

Table A-11a: Measured $M\left(R^{*}\right) / M(0)$ vs. $r^{*}$ for $10 \mu \mathrm{m}$ angular aluminum oxide blasted at $d=$ $30 \mathrm{~mm}$ for $60 \mathrm{~s}$ at a pressure of $300 \mathrm{kPa}$ and a flow rate of $4.20 \mathrm{~g} / \mathrm{min}$ using $0.76 \mathrm{~mm}$ round nozzle and an $\boldsymbol{r}_{c}$ of $\mathbf{0 . 6} \mathbf{~ m m}$ (corresponding to Figure 3.11).

\begin{tabular}{|c|c|c|c|c|c|c|}
\hline$r(\mathrm{~mm})$ & $r^{*}$ & $M(R)(\mathrm{g})$ & $M\left(R^{*}\right) / M(0)$ & $\begin{array}{c}M(R)(\mathrm{g}) \\
(\text { repeat })\end{array}$ & $\begin{array}{c}M\left(R^{*}\right) / M(0) \\
\text { (repeat) }\end{array}$ & $\begin{array}{c}\text { Standard } \\
\text { Deviation } \\
\left.\left(R^{*}\right) / M(0)\right)\end{array}$ \\
\hline 0 & 0 & 0.1005 & 1 & 0.0956 & 1 & 0 \\
\hline 0.6 & 0.02 & 0.1095 & 1.0896 & 0.1089 & 1.1391 & 0.02478 \\
\hline 1.2 & 0.04 & 0.0746 & 0.7423 & 0.0729 & 0.7626 & 0.01013 \\
\hline 1.8 & 0.06 & 0.022 & 0.2189 & 0.0194 & 0.2030 & 0.007988 \\
\hline 2.4 & 0.08 & 0.01 & 0.09950 & 0.0082 & 0.08577 & 0.006864 \\
\hline 3 & 0.1 & 0.0051 & 0.05075 & 0.0039 & 0.04079 & 0.004976 \\
\hline 3.6 & 0.12 & 0.0037 & 0.036812 & 0.0023 & 0.02406 & 0.006379 \\
\hline 4.2 & 0.14 & 0.0015 & 0.01493 & 0.0006 & 0.006276 & 0.004325 \\
\hline
\end{tabular}


Table A-11b: Measured flow rate for $10 \mu \mathrm{m}$ angular aluminum oxide blasted at $d=\mathbf{3 0} \mathrm{mm}$ for $60 \mathrm{~s}$ at a pressure of $300 \mathrm{kPa}$ using $0.76 \mathrm{~mm}$ round nozzle and an $r_{c}$ of $0.6 \mathrm{~mm}$ (corresponding to Figure 3.11).

\begin{tabular}{|c|c|c|c|c|}
\hline $\begin{array}{c}\text { Flow Rate } \\
\text { Measurement } \\
1(\mathrm{~g} / \mathrm{min})\end{array}$ & $\begin{array}{c}\text { Flow Rate } \\
\text { Measurement } \\
2(\mathrm{~g} / \mathrm{min})\end{array}$ & $\begin{array}{c}\text { Flow Rate } \\
\text { Measurement } \\
3(\mathrm{~g} / \mathrm{min})\end{array}$ & $\begin{array}{c}\text { Average Flow } \\
\text { Rate }(\mathrm{g} / \mathrm{min})\end{array}$ & $\begin{array}{c}\text { Standard } \\
\text { Deviation } \\
(\mathrm{g} / \mathrm{min})\end{array}$ \\
\hline 4.15 & 4.25 & 4.20 & 4.20 & 0.05 \\
\hline
\end{tabular}

Table A-12a: Measured $M\left(R^{*}\right) / M(0)$ vs. $r^{*}$ for $25 \mu \mathrm{m}$ angular aluminum oxide blasted at $d=$ $30 \mathrm{~mm}$ for $60 \mathrm{~s}$ at a pressure of $300 \mathrm{kPa}$ and a flow rate of $3.10 \mathrm{~g} / \mathrm{min}$ using $0.76 \mathrm{~mm}$ round nozzle and an $r_{c}$ of $0.23 \mathrm{~mm}$ (corresponding to Figure 3.12).

\begin{tabular}{|c|c|c|c|c|c|c|}
\hline$r(\mathrm{~mm})$ & $r^{*}$ & $M(R)(\mathrm{g})$ & $M\left(R^{*}\right) / M(0)$ & $\begin{array}{c}M(R)(\mathrm{g}) \\
\text { (repeat) }\end{array}$ & $\begin{array}{c}M\left(R^{*}\right) / M(0) \\
(\text { repeat })\end{array}$ & $\begin{array}{c}\text { Standard } \\
\text { Deviation } \\
\left(M\left(R^{*}\right) / M(0)\right)\end{array}$ \\
\hline 0 & 0 & 0.0286 & 1 & 0.0295 & 1 & 0 \\
\hline 0.3 & 0.01 & 0.0255 & 0.8916 & 0.0279 & 0.9458 & 0.02708 \\
\hline 0.6 & 0.02 & 0.0253 & 0.8846 & 0.0265 & 0.8983 & 0.006845 \\
\hline 1 & 0.0333 & 0.0218 & 0.7622 & 0.0209 & 0.7085 & 0.02688 \\
\hline 1.5 & 0.05 & 0.0164 & 0.5734 & 0.0171 & 0.5797 & 0.003117 \\
\hline 2 & 0.0667 & 0.0096 & 0.3357 & 0.0101 & 0.3424 & 0.003354 \\
\hline 2.5 & 0.0833 & 0.0045 & 0.1573 & 0.0057 & 0.1932 & 0.01794 \\
\hline 3 & 0.1 & 0.003 & 0.1049 & 0.0028 & 0.09491 & 0.00499 \\
\hline
\end{tabular}

Table A-12b: Measured flow rate for $25 \mu \mathrm{m}$ angular aluminum oxide blasted at $\boldsymbol{d}=\mathbf{3 0} \mathrm{mm}$ for $60 \mathrm{~s}$ at a pressure of $300 \mathrm{kPa}$ using $0.76 \mathrm{~mm}$ round nozzle and an $r_{c}$ of $0.23 \mathrm{~mm}$ (corresponding to Figure 3.12).

\begin{tabular}{|c|c|c|c|c|}
\hline $\begin{array}{c}\text { Flow Rate } \\
\text { Measurement } \\
1(\mathrm{~g} / \mathrm{min})\end{array}$ & $\begin{array}{c}\text { Flow Rate } \\
\text { Measurement } \\
2(\mathrm{~g} / \mathrm{min})\end{array}$ & $\begin{array}{c}\text { Flow Rate } \\
\text { Measurement } \\
3(\mathrm{~g} / \mathrm{min})\end{array}$ & $\begin{array}{c}\text { Average Flow } \\
\text { Rate }(\mathrm{g} / \mathrm{min})\end{array}$ & $\begin{array}{c}\text { Standard } \\
\text { Deviation } \\
(\mathrm{g} / \mathrm{min})\end{array}$ \\
\hline 3.19 & 2.98 & 3.12 & 3.10 & 0.09 \\
\hline
\end{tabular}


Table A-13a: Measured $M\left(R^{*}\right) / M(0)$ vs. $r^{*}$ for $50 \mu \mathrm{m}$ silica glass beads blasted at $d=20 \mathrm{~mm}$ for $90 \mathrm{~s}$ at a pressure of $300 \mathrm{kPa}$ and a flow rate of $1.34 \mathrm{~g} / \mathrm{min}$ using $0.76 \mathrm{~mm}$ round nozzle and an $r_{c}$ of $0.23 \mathrm{~mm}$ (corresponding to Figure 3.13).

\begin{tabular}{|c|c|c|c|c|c|c|}
\hline$r(\mathrm{~mm})$ & $r^{*}$ & $M(R)(\mathrm{g})$ & $M\left(R^{*}\right) / M(0)$ & $\begin{array}{c}M(R)(\mathrm{g}) \\
\text { (repeat) }\end{array}$ & $\begin{array}{c}M\left(R^{*}\right) / M(0) \\
\text { (repeat) }\end{array}$ & $\begin{array}{c}\text { Standard } \\
\text { Deviation } \\
\left(M\left(R^{*}\right) / M(0)\right)\end{array}$ \\
\hline 0 & 0 & 0.1043 & 1 & 0.1053 & 1 & 0 \\
\hline 0.3 & 0.015 & 0.1117 & 1.0709 & 0.1087 & 1.03229 & 0.01933 \\
\hline 0.6 & 0.03 & 0.091 & 0.8725 & 0.0933 & 0.8860 & 0.006778 \\
\hline 1 & 0.05 & 0.0648 & 0.6213 & 0.0675 & 0.6410 & 0.00987 \\
\hline 1.5 & 0.075 & 0.0444 & 0.4257 & 0.0473 & 0.4492 & 0.01175 \\
\hline 2 & 0.1 & 0.0236 & 0.2263 & 0.0251 & 0.2384 & 0.006048 \\
\hline 2.5 & 0.125 & 0.0146 & 0.1400 & 0.0155 & 0.1472 & 0.003609 \\
\hline 3 & 0.15 & 0.0052 & 0.04986 & 0.004 & 0.03799 & 0.005935 \\
\hline
\end{tabular}

Table A-13b: Measured flow rate for $50 \mu \mathrm{m}$ silica glass beads blasted at $d=20 \mathrm{~mm}$ for $90 \mathrm{~s}$ at a pressure of $300 \mathrm{kPa}$ using $0.76 \mathrm{~mm}$ round nozzle and an $r_{c}$ of $0.23 \mathrm{~mm}$ (corresponding to Figure 3.13).

\begin{tabular}{|c|c|c|c|c|}
\hline $\begin{array}{c}\text { Flow Rate } \\
\text { Measurement } \\
1(\mathrm{~g} / \mathrm{min})\end{array}$ & $\begin{array}{c}\text { Flow Rate } \\
\text { Measurement } \\
2(\mathrm{~g} / \mathrm{min})\end{array}$ & $\begin{array}{c}\text { Flow Rate } \\
\text { Measurement } \\
3(\mathrm{~g} / \mathrm{min})\end{array}$ & $\begin{array}{c}\text { Average Flow } \\
\text { Rate }(\mathrm{g} / \mathrm{min})\end{array}$ & $\begin{array}{c}\text { Standard } \\
\text { Deviation } \\
(\mathrm{g} / \mathrm{min})\end{array}$ \\
\hline 1.36 & 1.31 & 1.35 & 1.34 & 0.02 \\
\hline
\end{tabular}

Table A-14a: Measured $M\left(R^{*}\right) / M(0)$ vs. $r^{*}$ for $50 \mu \mathrm{m}$ silica glass beads blasted at $d=30 \mathrm{~mm}$ for $90 \mathrm{~s}$ at a pressure of $300 \mathrm{kPa}$ and a flow rate of $1.34 \mathrm{~g} / \mathrm{min}$ using $0.76 \mathrm{~mm}$ round nozzle and an $\boldsymbol{r}_{c}$ of $0.23 \mathrm{~mm}$ (corresponding to Figure 3.13).

\begin{tabular}{|c|c|c|c|c|c|c|}
\hline$r(\mathrm{~mm})$ & $r^{*}$ & $M(R)(\mathrm{g})$ & $M\left(R^{*}\right) / M(0)$ & $\begin{array}{c}M(R)(\mathrm{g}) \\
(\text { repeat })\end{array}$ & $\begin{array}{c}M\left(R^{*}\right) / M(0) \\
\text { (repeat) }\end{array}$ & $\begin{array}{c}\text { Standard } \\
\text { Deviation } \\
\left(M\left(R^{*}\right) / M(0)\right)\end{array}$ \\
\hline 0 & 0 & 0.0592 & 1 & 0.0587 & 1 & 0 \\
\hline 0.3 & 0.01 & 0.062 & 1.0473 & 0.0606 & 1.0323 & 0.0075 \\
\hline 0.6 & 0.02 & 0.058 & 0.9797 & 0.0579 & 0.9858 & 0.00302 \\
\hline 1 & 0.0333 & 0.0525 & 0.8868 & 0.051 & 0.8688 & 0.00903 \\
\hline 1.5 & 0.05 & 0.045 & 0.7601 & 0.0461 & 0.7857 & 0.01276 \\
\hline 2 & 0.0667 & 0.0315 & 0.5321 & 0.03 & 0.5108 & 0.01064 \\
\hline 2.5 & 0.0833 & 0.0197 & 0.3328 & 0.0211 & 0.3599 & 0.01356 \\
\hline 3 & 0.1 & 0.0128 & 0.2162 & 0.0106 & 0.1799 & 0.01817 \\
\hline
\end{tabular}


Table A-14b: Measured flow rate for $50 \mu \mathrm{m}$ silica glass beads blasted at $d=\mathbf{3 0} \mathrm{mm}$ for $90 \mathrm{~s}$ at a pressure of $300 \mathrm{kPa}$ using $0.76 \mathrm{~mm}$ round nozzle and an $r_{c}$ of $0.23 \mathrm{~mm}$ (corresponding to Figure 3.13).

\begin{tabular}{|c|c|c|c|c|}
\hline $\begin{array}{c}\text { Flow Rate } \\
\text { Measurement } \\
1(\mathrm{~g} / \mathrm{min})\end{array}$ & $\begin{array}{c}\text { Flow Rate } \\
\text { Measurement } \\
2(\mathrm{~g} / \mathrm{min})\end{array}$ & $\begin{array}{c}\text { Flow Rate } \\
\text { Measurement } \\
3(\mathrm{~g} / \mathrm{min})\end{array}$ & $\begin{array}{c}\text { Average Flow } \\
\text { Rate }(\mathrm{g} / \mathrm{min})\end{array}$ & $\begin{array}{c}\text { Standard } \\
\text { Deviation } \\
(\mathrm{g} / \mathrm{min})\end{array}$ \\
\hline 1.36 & 1.31 & 1.35 & 1.34 & 0.02 \\
\hline
\end{tabular}

Table A-15a: Measured $M\left(X^{*}\right) / M(0)$ vs. $x^{*}$ for $50 \mu \mathrm{m}$ silica glass beads blasted at $d=40 \mathrm{~mm}$ for $120 \mathrm{~s}$ at a pressure of $200 \mathrm{kPa}$ and a flow rate of $1.21 \mathrm{~g} / \mathrm{min}$ using $3.2 \times 0.2 \mathrm{~mm}$ rectangular nozzle and an $r_{c}$ of $0.23 \mathrm{~mm}$ (scanning direction along $0.2 \mathrm{~mm}$ ) (corresponding to Figure 3.14).

\begin{tabular}{|c|c|c|c|c|c|c|}
\hline$x(\mathrm{~mm})$ & $x^{*}$ & $M(X)(\mathrm{g})$ & $M\left(X^{*}\right) / M(0)$ & $\begin{array}{c}M(X)(\mathrm{g}) \\
(\text { repeat })\end{array}$ & $\begin{array}{c}M\left(X^{*}\right) / M(0) \\
\text { (repeat) }\end{array}$ & $\begin{array}{c}\text { Standard } \\
\text { Deviation } \\
\left.\left(X^{*}\right) / M(0)\right)\end{array}$ \\
\hline 0 & 0 & 0.0383 & 1 & 0.0378 & 1 & 0 \\
\hline 0.3 & 0.0075 & 0.0364 & 0.9504 & 0.0389 & 1.0291 & 0.03935 \\
\hline 0.6 & 0.015 & 0.0414 & 1.0809 & 0.0427 & 1.1296 & 0.02435 \\
\hline 0.9 & 0.0225 & 0.0362 & 0.9452 & 0.0399 & 1.0556 & 0.05519 \\
\hline 1.2 & 0.03 & 0.0401 & 1.0470 & 0.0412 & 1.0899 & 0.02148 \\
\hline 1.5 & 0.0375 & 0.0462 & 1.2063 & 0.0444 & 1.1746 & 0.01583 \\
\hline 2 & 0.05 & 0.0449 & 1.1723 & 0.0461 & 1.2196 & 0.02363 \\
\hline 2.5 & 0.0625 & 0.0479 & 1.2507 & 0.0481 & 1.2725 & 0.01092 \\
\hline 3 & 0.075 & 0.0408 & 1.0653 & 0.0289 & 0.7646 & 0.04983 \\
\hline 3.5 & 0.0875 & 0.0266 & 0.6945 & 0.0237 & 0.6270 & 0.03377 \\
\hline 4 & 0.1 & 0.0219 & 0.5718 & 0.0189 & 0.5 & 0.0359 \\
\hline 4.5 & 0.1125 & 0.015 & 0.3916 & 0.0141 & 0.3730 & 0.009315 \\
\hline 5 & 0.125 & 0.0094 & 0.2454 & 0.0101 & 0.2672 & 0.01089 \\
\hline 5.5 & 0.1375 & 0.0067 & 0.1749 & 0.0075 & 0.1984 & 0.01174 \\
\hline 6 & 0.15 & 0.0038 & 0.09922 & 0.0057 & 0.1508 & 0.02579 \\
\hline 6.5 & 0.1625 & 0.002 & 0.05222 & 0.0036 & 0.09524 & 0.02151 \\
\hline
\end{tabular}

Table A-15b: Measured flow rate for $50 \mu \mathrm{m}$ silica glass beads blasted at $d=\mathbf{4 0} \mathrm{mm}$ for 120 $s$ at a pressure of $200 \mathrm{kPa}$ using $3.2 \times 0.2 \mathrm{~mm}$ rectangular nozzle and an $r_{c}$ of $0.23 \mathrm{~mm}$ (scanning direction along $0.2 \mathrm{~mm}$ ) (corresponding to Figure 3.14).

\begin{tabular}{|c|c|c|c|c|}
\hline $\begin{array}{c}\text { Flow Rate } \\
\text { Measurement } \\
1(\mathrm{~g} / \mathrm{min})\end{array}$ & $\begin{array}{c}\text { Flow Rate } \\
\text { Measurement } \\
2(\mathrm{~g} / \mathrm{min})\end{array}$ & $\begin{array}{c}\text { Flow Rate } \\
\text { Measurement } \\
3(\mathrm{~g} / \mathrm{min})\end{array}$ & $\begin{array}{c}\text { Average Flow } \\
\text { Rate }(\mathrm{g} / \mathrm{min})\end{array}$ & $\begin{array}{c}\text { Standard } \\
\text { Deviation } \\
(\mathrm{g} / \mathrm{min})\end{array}$ \\
\hline 1.24 & 1.17 & 1.22 & 1.21 & 0.03 \\
\hline
\end{tabular}


Table A-16a: Measured $M\left(Y^{*}\right) / M(0)$ vs. $y^{*}$ for $50 \mu \mathrm{m}$ silica glass beads blasted at $d=40 \mathrm{~mm}$ for $60 \mathrm{~s}$ at a pressure of $200 \mathrm{kPa}$ and a flow rate of $1.21 \mathrm{~g} / \mathrm{min}$ using $3.2 \times 0.2 \mathrm{~mm}$ rectangular nozzle and an $\boldsymbol{r}_{c}$ of $0.23 \mathrm{~mm}$ (scanning direction along $3.2 \mathrm{~mm}$ ) (corresponding to Figure 3.14).

\begin{tabular}{|c|c|c|c|c|c|c|}
\hline$y(\mathrm{~mm})$ & $y^{*}$ & $M(Y)(\mathrm{g})$ & $M\left(Y^{*}\right) / M(0)$ & $\begin{array}{c}M(Y)(\mathrm{g}) \\
\text { (repeat) }\end{array}$ & $\begin{array}{c}M\left(Y^{*}\right) / M(0) \\
\text { (repeat) }\end{array}$ & $\begin{array}{c}\text { Standard } \\
\text { Deviation } \\
\left(M\left(Y^{*}\right) / M(0)\right)\end{array}$ \\
\hline 0 & 0 & 0.0398 & 1 & 0.0401 & 1 & 0 \\
\hline 0.5 & 0.0125 & 0.0396 & 0.9950 & 0.0395 & 0.9853 & 0.004812 \\
\hline 1 & 0.025 & 0.0392 & 0.9849 & 0.0392 & 0.9766 & 0.004171 \\
\hline 1.5 & 0.0375 & 0.0307 & 0.7714 & 0.0309 & 0.7699 & 0.000742 \\
\hline 2 & 0.05 & 0.0276 & 0.6935 & 0.0281 & 0.7007 & 0.003611 \\
\hline 2.5 & 0.0625 & 0.0197 & 0.4950 & 0.0201 & 0.5010 & 0.003015 \\
\hline 3 & 0.075 & 0.0091 & 0.2286 & 0.0094 & 0.2346 & 0.002963 \\
\hline 3.5 & 0.0875 & 0.0068 & 0.1709 & 0.0076 & 0.1890 & 0.009061 \\
\hline
\end{tabular}

Table A-16b: Measured flow rate for $50 \mu \mathrm{m}$ silica glass beads blasted at $d=40 \mathrm{~mm}$ for $60 \mathrm{~s}$ at a pressure of $200 \mathrm{kPa}$ using $3.2 \times 0.2 \mathrm{~mm}$ rectangular nozzle and an $r_{c}$ of $0.23 \mathrm{~mm}$ (scanning direction along $3.2 \mathrm{~mm}$ ) (corresponding to Figure 3.14).

\begin{tabular}{|c|c|c|c|c|}
\hline $\begin{array}{c}\text { Flow Rate } \\
\text { Measurement } \\
1(\mathrm{~g} / \mathrm{min})\end{array}$ & $\begin{array}{c}\text { Flow Rate } \\
\text { Measurement } \\
2(\mathrm{~g} / \mathrm{min})\end{array}$ & $\begin{array}{c}\text { Flow Rate } \\
\text { Measurement } \\
3(\mathrm{~g} / \mathrm{min})\end{array}$ & $\begin{array}{c}\text { Average Flow } \\
\text { Rate }(\mathrm{g} / \mathrm{min})\end{array}$ & $\begin{array}{c}\text { Standard } \\
\text { Deviation } \\
(\mathrm{g} / \mathrm{min})\end{array}$ \\
\hline 1.24 & 1.17 & 1.22 & 1.21 & 0.03 \\
\hline
\end{tabular}


Table A-17a: Measured $M\left(X^{*}\right) / M(0)$ vs. $x^{*}$ for $50 \mu \mathrm{m}$ silica glass beads blasted at $d=40 \mathrm{~mm}$ for $120 \mathrm{~s}$ at a pressure of $200 \mathrm{kPa}$ and a flow rate of $1.78 \mathrm{~g} / \mathrm{min}$ using $3.8 \times 0.3 \mathrm{~mm}$ rectangular nozzle and an $\boldsymbol{r}_{c}$ of $0.23 \mathrm{~mm}$ (scanning direction along $0.3 \mathrm{~mm}$ ) (corresponding to Figure 3.15).

\begin{tabular}{|c|c|c|c|c|c|c|}
\hline$x(\mathrm{~mm})$ & $x^{*}$ & $M(X)(\mathrm{g})$ & $M\left(X^{*}\right) / M(0)$ & $\begin{array}{c}M(X)(\mathrm{g}) \\
\text { (repeat) }\end{array}$ & $\begin{array}{c}M\left(X^{*}\right) / M(0) \\
\text { (repeat) }\end{array}$ & $\begin{array}{c}\text { Standard } \\
\text { Deviation } \\
\left(M\left(X^{*}\right) / M(0)\right)\end{array}$ \\
\hline 0 & 0 & 0.0419 & 1 & 0.0435 & 1 & 0 \\
\hline 0.5 & 0.0125 & 0.0386 & 0.9212 & 0.0401 & 0.9218 & 0.0002991 \\
\hline 1 & 0.025 & 0.0434 & 1.0358 & 0.0446 & 1.0253 & 0.005256 \\
\hline 1.5 & 0.0375 & 0.0468 & 1.1169 & 0.0473 & 1.0874 & 0.01479 \\
\hline 2 & 0.05 & 0.0489 & 1.1671 & 0.0503 & 1.1563 & 0.005371 \\
\hline 2.5 & 0.0625 & 0.0393 & 0.9379 & 0.0385 & 0.8851 & 0.02643 \\
\hline 3 & 0.075 & 0.0327 & 0.7804 & 0.0341 & 0.7839 & 0.001739 \\
\hline 3.5 & 0.0875 & 0.0208 & 0.4964 & 0.0246 & 0.5655 & 0.03455 \\
\hline 4 & 0.1 & 0.0173 & 0.4129 & 0.023 & 0.5287 & 0.05792 \\
\hline 4.5 & 0.1125 & 0.0148 & 0.3532 & 0.0161 & 0.3701 & 0.008446 \\
\hline 5 & 0.125 & 0.0112 & 0.2673 & 0.0103 & 0.2368 & 0.01526 \\
\hline 5.5 & 0.1375 & 0.008 & 0.1909 & 0.0093 & 0.2138 & 0.01143 \\
\hline 6 & 0.15 & 0.0066 & 0.1575 & 0.0051 & 0.1172 & 0.02014 \\
\hline 6.5 & 0.1625 & 0.0055 & 0.1313 & 0.0043 & 0.09885 & 0.01621 \\
\hline
\end{tabular}

Table A-17b: Measured flow rate for $50 \mu \mathrm{m}$ silica glass beads blasted at $\boldsymbol{d}=\mathbf{4 0} \mathrm{mm}$ for 120 $s$ at a pressure of $200 \mathrm{kPa}$ using $3.8 \times 0.3 \mathrm{~mm}$ rectangular nozzle and an $r_{c}$ of $0.23 \mathrm{~mm}$ (scanning direction along $0.3 \mathrm{~mm}$ ) (corresponding to Figure 3.15).

\begin{tabular}{|c|c|c|c|c|}
\hline $\begin{array}{c}\text { Flow Rate } \\
\text { Measurement } \\
1(\mathrm{~g} / \mathrm{min})\end{array}$ & $\begin{array}{c}\text { Flow Rate } \\
\text { Measurement } \\
2(\mathrm{~g} / \mathrm{min})\end{array}$ & $\begin{array}{c}\text { Flow Rate } \\
\text { Measurement } \\
3(\mathrm{~g} / \mathrm{min})\end{array}$ & $\begin{array}{c}\text { Average Flow } \\
\text { Rate }(\mathrm{g} / \mathrm{min})\end{array}$ & $\begin{array}{c}\text { Standard } \\
\text { Deviation } \\
(\mathrm{g} / \mathrm{min})\end{array}$ \\
\hline 1.72 & 1.85 & 1.77 & 1.78 & 0.05 \\
\hline
\end{tabular}


Table A-18a: Measured $M\left(Y^{*}\right) / M(0)$ vs. $y^{*}$ for $50 \mu \mathrm{m}$ silica glass beads blasted at $d=40 \mathrm{~mm}$ for $120 \mathrm{~s}$ at a pressure of $200 \mathrm{kPa}$ and a flow rate of $1.78 \mathrm{~g} / \mathrm{min}$ using $3.8 \times 0.3 \mathrm{~mm}$ rectangular nozzle and an $r_{c}$ of $0.23 \mathrm{~mm}$ (scanning direction along $3.8 \mathrm{~mm}$ ) (corresponding to Figure 3.15).

\begin{tabular}{|c|c|c|c|c|c|c|}
\hline$y(\mathrm{~mm})$ & $y^{*}$ & $M(Y)(\mathrm{g})$ & $M\left(Y^{*}\right) / M(0)$ & $\begin{array}{c}M(Y)(\mathrm{g}) \\
\text { (repeat) }\end{array}$ & $\begin{array}{c}M\left(Y^{*}\right) / M(0) \\
\text { (repeat) }\end{array}$ & $\begin{array}{c}\text { Standard } \\
\text { Deviation } \\
\left(M\left(Y^{*}\right) / M(0)\right)\end{array}$ \\
\hline 0 & 0 & 0.0422 & 1 & 0.0429 & 1 & 0 \\
\hline 0.3 & 0.0075 & 0.0418 & 0.9905 & 0.0421 & 0.9814 & 0.004585 \\
\hline 0.6 & 0.015 & 0.0401 & 0.9502 & 0.0411 & 0.9580 & 0.003902 \\
\hline 1 & 0.025 & 0.0385 & 0.9123 & 0.0374 & 0.8718 & 0.02026 \\
\hline 1.5 & 0.0375 & 0.0339 & 0.8033 & 0.0354 & 0.8252 & 0.01093 \\
\hline 2 & 0.05 & 0.0281 & 0.6659 & 0.0285 & 0.6643 & 0.000771 \\
\hline 2.5 & 0.0625 & 0.025 & 0.5924 & 0.0251 & 0.5851 & 0.003668 \\
\hline 3 & 0.075 & 0.0189 & 0.44787 & 0.0196 & 0.4569 & 0.004505 \\
\hline 3.5 & 0.0875 & 0.0138 & 0.3270 & 0.0142 & 0.3310 & 0.001994 \\
\hline 4 & 0.1 & 0.0086 & 0.2038 & 0.0075 & 0.1748 & 0.01448 \\
\hline
\end{tabular}

Table A-18b: Measured flow rate for $50 \mu \mathrm{m}$ silica glass beads blasted at $\boldsymbol{d}=\mathbf{4 0} \mathrm{mm}$ for 120 $s$ at a pressure of $200 \mathrm{kPa}$ using $3.8 \times 0.3 \mathrm{~mm}$ rectangular nozzle and an $r_{c}$ of $0.23 \mathrm{~mm}$ (scanning direction along $3.8 \mathrm{~mm}$ ) (corresponding to Figure 3.15).

\begin{tabular}{|c|c|c|c|c|}
\hline $\begin{array}{c}\text { Flow Rate } \\
\text { Measurement } \\
1(\mathrm{~g} / \mathrm{min})\end{array}$ & $\begin{array}{c}\text { Flow Rate } \\
\text { Measurement } \\
2(\mathrm{~g} / \mathrm{min})\end{array}$ & $\begin{array}{c}\text { Flow Rate } \\
\text { Measurement } \\
3(\mathrm{~g} / \mathrm{min})\end{array}$ & $\begin{array}{c}\text { Average Flow } \\
\text { Rate }(\mathrm{g} / \mathrm{min})\end{array}$ & $\begin{array}{c}\text { Standard } \\
\text { Deviation } \\
(\mathrm{g} / \mathrm{min})\end{array}$ \\
\hline 1.72 & 1.85 & 1.77 & 1.78 & 0.05 \\
\hline
\end{tabular}




\section{Appendix B Measured $\delta\left(r^{*}\right) / \delta(0)$ and Calculated $v^{*}\left(r^{*}\right)$ Values, along with Measured $k$ Values (Selected Cases) used in Obtaining $v^{*}\left(r^{*}\right)$ (Corresponding to Section 4.3)}

Table B-1a: Measured $\delta\left(r^{*}\right) / \delta(0)$ (erosion time $=10$ s) and calculated $v^{*}\left(r^{*}\right)$ vs. $r^{*}$ (corresponding to Figure 3.4 of Section 3.3, analysis of Section 4.2, and Figure 4.5 of Section 4.3) using $k=1.57$ (Figure 4.2) for $10 \mu \mathrm{m}$ angular aluminum oxide blasted at $d=30$ $\mathrm{mm}$ at a pressure of $200 \mathrm{kPa}$ and a flow rate of $3.97 \mathrm{~g} / \mathrm{min}$ using $0.76 \mathrm{~mm}$ round nozzle.

\begin{tabular}{|c|c|c|c|c|c|}
\hline$r(\mathrm{~mm})$ & $r^{*}$ & $\begin{array}{c}\text { Avg. } \\
M\left(R^{*}\right) / M(0)\end{array}$ & $\delta(r)(\mathrm{mm})$ & $\delta\left(r^{*}\right) / \delta(0)$ & $\begin{array}{c}\delta(r)(\mathrm{mm}) \\
(\text { repeat })\end{array}$ \\
\hline 0 & 0 & 1 & -0.134 & 1 & -0.148 \\
\hline 0.6 & 0.02 & 1.1866 & -0.0924 & 0.6896 & -0.104 \\
\hline 1.2 & 0.04 & 1.2278 & -0.0358 & 0.2672 & -0.0443 \\
\hline 1.8 & 0.06 & 0.7613 & -0.00927 & 0.06918 & -0.0146 \\
\hline 2.4 & 0.08 & 0.3300 & -0.00132 & 0.009851 & -0.00352 \\
\hline 3 & 0.1 & 0.1535 & -0.000692 & 0.005164 & -0.000636 \\
\hline 3.6 & 0.12 & 0.1009 & -0.000087 & 0.0006493 & -0.0000446 \\
\hline 4.2 & 0.14 & 0.07139 & -0.0000264 & 0.0001970 & -0.000000813 \\
\hline
\end{tabular}

Table B-1a...Continued.

\begin{tabular}{|c|c|c|c|}
\hline $\begin{array}{c}\delta\left(r^{*}\right) / \delta(0) \\
\text { (repeat) }\end{array}$ & $\begin{array}{c}\text { Avg. } \\
\delta\left(r^{*}\right) / \delta(0)\end{array}$ & $\begin{array}{c}\text { Standard } \\
\text { Deviation } \\
\left(\delta\left(r^{*}\right) / \delta(0)\right)\end{array}$ & $v^{*}\left(r^{*}\right)$ \\
\hline 1 & 1 & 0 & 1 \\
\hline 0.7027 & 0.6961 & 0.006575 & 0.712 \\
\hline 0.2993 & 0.2832 & 0.01608 & 0.3929 \\
\hline 0.09864 & 0.08391 & 0.01473 & 0.2455 \\
\hline 0.02378 & 0.01682 & 0.006967 & 0.1502 \\
\hline 0.004297 & 0.004731 & 0.0004334 & 0.1090 \\
\hline 0.0003014 & 0.0004753 & 0.000174 & 0.03295 \\
\hline 0.000005493 & 0.0001013 & 0.00009576 & 0.01534 \\
\hline
\end{tabular}


Table B-1b: Measured normalized average erosion rate, $E^{*}$, as a function of $\sin (\alpha)$ (corresponding to Figure 4.2 and Table B-1a). Best fit velocity exponent $k=1.57$. Erosion of Borofloat glass by $10 \mu \mathrm{m}$ aluminum oxide particles blasted at $d=30 \mathrm{~mm}$ for $60 \mathrm{~s}$ at a pressure of $200 \mathrm{kPa}$ and a flow rate of $3.97 \mathrm{~g} / \mathrm{min}$ using $0.76 \mathrm{~mm}$ round nozzle.

\begin{tabular}{|c|c|c|c|c|c|}
\hline$\alpha$ (deg.) & $\sin (\alpha)$ & $\begin{array}{c}\text { Mass Loss of } \\
\text { Substrate/min } \\
(\mathrm{g} / \mathrm{min})\end{array}$ & $\begin{array}{c}\text { Mass Loss of } \\
\text { Substrate/min } \\
(\mathrm{g} / \mathrm{min}) \\
(\text { repeat })\end{array}$ & $\begin{array}{c}\text { Standard } \\
\text { Deviation } \\
(\text { Mass loss of } \\
\text { Substrate/min }) \\
(\mathrm{g} / \mathrm{min})\end{array}$ & $\begin{array}{c}\text { Avg. Mass } \\
\text { Loss of } \\
\text { Substrate/min }) \\
(\mathrm{g} / \mathrm{min})\end{array}$ \\
\hline 90 & 1 & 0.0074 & 0.008 & 0.0003 & 0.0077 \\
\hline 72.5 & 0.9537 & 0.0063 & 0.008 & 0.00085 & 0.00715 \\
\hline 60 & 0.8660 & 0.0063 & 0.0065 & 0.0001 & 0.0064 \\
\hline 45 & 0.7071 & 0.0035 & 0.0043 & 0.0004 & 0.0039 \\
\hline 30 & 0.5 & 0.0029 & 0.0025 & 0.0002 & 0.0027 \\
\hline
\end{tabular}

Table B-1b...Continued.

\begin{tabular}{|c|c|}
\hline $\begin{array}{c}E \text { (using flow } \\
\text { rate from } \\
\text { Figure 3.4) }\end{array}$ & $\begin{array}{c}E^{*} \\
\text { (normalized } \\
\text { with respect } \\
\text { to } E \text { at } \alpha=90^{\circ} \text { ) }\end{array}$ \\
\hline 0.001925 & 1 \\
\hline 0.001788 & 0.9286 \\
\hline 0.0016 & 0.8312 \\
\hline 0.000975 & 0.5065 \\
\hline 0.000675 & 0.3506 \\
\hline
\end{tabular}

Table B-2: Measured $\delta\left(r^{*}\right) / \delta(0)$ (erosion time $=10$ s) and calculated $v^{*}\left(r^{*}\right)$ vs. $r^{*}$ (corresponding to Figure 3.5 of Section 3.3, analysis of Section 4.2, and Figure 4.6 of Section 4.3) using $k=1.43$ (from [25]) for $25 \mu \mathrm{m}$ angular aluminum oxide blasted at $d=30$ $\mathrm{mm}$ at a pressure of $200 \mathrm{kPa}$ and a flow rate of $2.83 \mathrm{~g} / \mathrm{min}$ using $0.76 \mathrm{~mm}$ round nozzle.

\begin{tabular}{|c|c|c|c|c|c|}
\hline$r(\mathrm{~mm})$ & $r^{*}$ & $\begin{array}{c}\text { Avg. } \\
M\left(R^{*}\right) / M(0)\end{array}$ & $\delta(r)(\mathrm{mm})$ & $\delta\left(r^{*}\right) / \delta(0)$ & $\begin{array}{c}\delta(r)(\mathrm{mm}) \\
\text { (repeat) }\end{array}$ \\
\hline 0 & 0 & 1 & -0.14 & 1 & -0.131 \\
\hline 0.3 & 0.01 & 1.083 & -0.127 & 0.9071 & -0.122 \\
\hline 0.6 & 0.02 & 0.8086 & -0.108 & 0.7714 & -0.109 \\
\hline 1 & 0.0333 & 0.7526 & -0.087 & 0.6214 & -0.089 \\
\hline 1.5 & 0.05 & 0.5856 & -0.0562 & 0.4014 & -0.0585 \\
\hline 2 & 0.0667 & 0.3846 & -0.0319 & 0.2279 & -0.0398 \\
\hline 2.5 & 0.0833 & 0.2469 & -0.0163 & 0.1164 & -0.0179 \\
\hline 3 & 0.1 & 0.1058 & -0.00425 & 0.03035 & -0.00465 \\
\hline
\end{tabular}


Table B-2...Continued.

\begin{tabular}{|c|c|c|c|}
\hline $\begin{array}{c}\delta\left(r^{*}\right) / \delta(0) \\
\text { (repeat) }\end{array}$ & $\begin{array}{c}\text { Avg. } \\
\delta\left(r^{*}\right) / \delta(0)\end{array}$ & $\begin{array}{c}\text { Standard } \\
\text { Deviation } \\
\left(\delta\left(r^{*}\right) / \delta(0)\right)\end{array}$ & $v^{*}\left(r^{*}\right)$ \\
\hline 1 & 1 & 0 & 1 \\
\hline 0.9313 & 0.9192 & 0.01207 & 0.8916 \\
\hline 0.8321 & 0.8017 & 0.03032 & 0.9941 \\
\hline 0.6794 & 0.6504 & 0.02898 & 0.903 \\
\hline 0.4466 & 0.424 & 0.02257 & 0.7979 \\
\hline 0.3038 & 0.2658 & 0.03798 & 0.7724 \\
\hline 0.1366 & 0.1265 & 0.01011 & 0.6265 \\
\hline 0.0355 & 0.03293 & 0.00257 & 0.4422 \\
\hline
\end{tabular}

Table B-3: Measured $\delta\left(r^{*}\right) / \delta(0)$ (erosion time $=180$ s) and calculated $v^{*}\left(r^{*}\right)$ vs. $r^{*}$ (corresponding to Figure 3.6 of Section 3.3, analysis of Section 4.2, and Figure 4.7 of Section 4.3) using $k=1.6$ and $k=2.7$ for $50 \mu \mathrm{m}$ silica glass beads blasted at $d=20 \mathrm{~mm}$ at a pressure of $200 \mathrm{kPa}$ and a flow rate of $1.17 \mathrm{~g} / \mathrm{min}$ using $0.76 \mathrm{~mm}$ round nozzle.

\begin{tabular}{|c|c|c|c|c|c|}
\hline$r(\mathrm{~mm})$ & $r^{*}$ & $\begin{array}{c}\text { Avg. } \\
M\left(R^{*}\right) / M(0)\end{array}$ & $\delta(r)(\mathrm{mm})$ & $\delta\left(r^{*}\right) / \delta(0)$ & $\begin{array}{c}\delta(r)(\mathrm{mm}) \\
\text { (repeat) }\end{array}$ \\
\hline 0 & 0 & 1 & -0.0416 & 1 & -0.028 \\
\hline 0.3 & 0.015 & 0.9170 & -0.0338 & 0.8125 & -0.0234 \\
\hline 0.6 & 0.03 & 0.7792 & -0.0245 & 0.5889 & -0.0179 \\
\hline 1 & 0.05 & 0.4219 & -0.0128 & 0.3077 & -0.00939 \\
\hline 1.5 & 0.075 & 0.2447 & -0.00626 & 0.1505 & -0.00403 \\
\hline 2 & 0.1 & 0.1435 & -0.00233 & 0.05601 & -0.000844 \\
\hline 2.5 & 0.125 & 0.07173 & -0.000272 & 0.006539 & -0.0000855 \\
\hline 3 & 0.15 & 0.03235 & -0.0000479 & 0.001151 & -0.000216 \\
\hline
\end{tabular}

Table B-3...Continued.

\begin{tabular}{|c|c|c|c|c|}
\hline $\begin{array}{c}\delta\left(r^{*}\right) / \delta(0) \\
(\text { repeat })\end{array}$ & $\begin{array}{c}\text { Avg. } \\
\delta\left(r^{*}\right) / \delta(0)\end{array}$ & $\begin{array}{c}\text { Standard } \\
\text { Deviation } \\
\left(\delta\left(r^{*}\right) / \delta(0)\right)\end{array}$ & $\begin{array}{c}v^{*}\left(r^{*}\right) \\
(k=1.6)\end{array}$ & $\begin{array}{c}v^{*}\left(r^{*}\right) \\
(k=2.7)\end{array}$ \\
\hline 1 & 1 & 0 & 1 & 1 \\
\hline 0.8357 & 0.8241 & 0.01161 & 0.9441 & 0.9665 \\
\hline 0.6393 & 0.6141 & 0.02517 & 0.872 & 0.922 \\
\hline 0.3354 & 0.3215 & 0.01383 & 0.8442 & 0.9045 \\
\hline 0.1439 & 0.1472 & 0.003276 & 0.728 & 0.8285 \\
\hline 0.03014 & 0.04308 & 0.01293 & 0.4654 & 0.6356 \\
\hline 0.003054 & 0.004796 & 0.001742 & 0.1799 & 0.3619 \\
\hline 0.007714 & 0.004433 & 0.003281 & 0.2707 & 0.461 \\
\hline
\end{tabular}


Table B-4: Measured $\delta\left(r^{*}\right) / \delta(0)$ (erosion time $=10$ s) and calculated $v^{*}\left(r^{*}\right)$ vs. $r^{*}$ (corresponding to Figure 3.7 of Section 3.3, analysis of Section 4.2, and Figure 4.8 of Section 4.3) using $k=1.43$ (from [25]) for $25 \mu \mathrm{m}$ angular aluminum oxide blasted at $d=10$ $\mathrm{mm}$ at a pressure of $200 \mathrm{kPa}$ and a flow rate of $0.36 \mathrm{~g} / \mathrm{min}$ using $0.46 \mathrm{~mm} \mathrm{H}$.P. round nozzle.

\begin{tabular}{|c|c|c|c|c|c|}
\hline$r(\mathrm{~mm})$ & $r^{*}$ & $\begin{array}{c}\text { Avg. } \\
M\left(R^{*}\right) / M(0)\end{array}$ & $\delta(r)(\mathrm{mm})$ & $\delta\left(r^{*}\right) / \delta(0)$ & $\begin{array}{c}\delta(r)(\mathrm{mm}) \\
(\mathrm{repeat})\end{array}$ \\
\hline 0 & 0 & 1 & -0.108 & 1 & -0.125 \\
\hline 0.3 & 0.03 & 1.4153 & -0.0773 & 0.7157 & -0.0938 \\
\hline 0.6 & 0.06 & 0.8782 & -0.0413 & 0.3824 & -0.0521 \\
\hline 0.9 & 0.09 & 0.4066 & -0.0163 & 0.1509 & -0.0217 \\
\hline 1.2 & 0.12 & 0.1185 & -0.00479 & 0.04435 & -0.00623 \\
\hline 1.5 & 0.15 & 0.04150 & -0.00104 & 0.009630 & -0.00157 \\
\hline 1.8 & 0.18 & 0.01580 & -0.000298 & 0.002760 & -0.00025 \\
\hline 2.1 & 0.21 & 0.005974 & -0.00000735 & 0.00006806 & -0.000001 \\
\hline
\end{tabular}

Table B-4...Continued.

\begin{tabular}{|c|c|c|c|}
\hline $\begin{array}{c}\delta\left(r^{*}\right) / \delta(0) \\
\text { (repeat) }\end{array}$ & $\begin{array}{c}\text { Avg. } \\
\delta\left(r^{*}\right) / \delta(0)\end{array}$ & $\begin{array}{c}\text { Standard } \\
\text { Deviation } \\
\left(\delta\left(r^{*}\right) / \delta(0)\right)\end{array}$ & $v^{*}\left(r^{*}\right)$ \\
\hline 1 & 1 & 0 & 1 \\
\hline 0.7504 & 0.7331 & 0.01733 & 0.6313 \\
\hline 0.4168 & 0.3996 & 0.01720 & 0.5766 \\
\hline 0.1736 & 0.1623 & 0.01134 & 0.5261 \\
\hline 0.04984 & 0.04710 & 0.002744 & 0.5247 \\
\hline 0.01256 & 0.01109 & 0.001465 & 0.3975 \\
\hline 0.002 & 0.002380 & 0.0003796 & 0.2661 \\
\hline 0.000008 & 0.00003803 & 0.00003003 & 0.02912 \\
\hline
\end{tabular}

Table B-5: Measured $\delta\left(r^{*}\right) / \delta(0)$ (erosion time $=2$ s) and calculated $v^{*}\left(r^{*}\right)$ vs. $r^{*}$ (corresponding to Figure 3.8 of Section 3.3, analysis of Section 4.2, and Figure 4.9 of Section 4.3) using $k=1.43$ (from [25]) for $25 \mu \mathrm{m}$ angular aluminum oxide blasted at $d=30$ $\mathrm{mm}$ at a pressure of $200 \mathrm{kPa}$ and a flow rate of $4.75 \mathrm{~g} / \mathrm{min}$ using $1.5 \mathrm{~mm}$ H.P. round nozzle.

\begin{tabular}{|c|c|c|c|c|c|}
\hline$r(\mathrm{~mm})$ & $r^{*}$ & $\begin{array}{c}\text { Avg. } \\
M\left(R^{*}\right) / M(0)\end{array}$ & $\delta(r)(\mathrm{mm})$ & $\delta\left(r^{*}\right) / \delta(0)$ & $\begin{array}{c}\delta(r)(\mathrm{mm}) \\
(\mathrm{repeat})\end{array}$ \\
\hline 0 & 0 & 1 & -0.157 & 1 & -0.154 \\
\hline 0.5 & 0.0167 & 0.7852 & -0.136 & 0.8662 & -0.129 \\
\hline 1 & 0.0333 & 0.6122 & -0.0943 & 0.6006 & -0.0871 \\
\hline 1.5 & 0.05 & 0.3027 & -0.0532 & 0.3388 & -0.0488 \\
\hline 2 & 0.0667 & 0.1676 & -0.0261 & 0.1662 & -0.0237 \\
\hline 2.5 & 0.0833 & 0.08684 & -0.0116 & 0.07389 & -0.0105 \\
\hline 3 & 0.1 & 0.06861 & -0.00474 & 0.03019 & -0.00358 \\
\hline 3.5 & 0.1167 & 0.02961 & -0.00134 & 0.008535 & -0.000477 \\
\hline
\end{tabular}


Table B-5...Continued.

\begin{tabular}{|c|c|c|c|}
\hline $\begin{array}{c}\delta\left(r^{*}\right) / \delta(0) \\
\text { (repeat) }\end{array}$ & $\begin{array}{c}\text { Avg. } \\
\delta\left(r^{*}\right) / \delta(0)\end{array}$ & $\begin{array}{c}\text { Standard } \\
\text { Deviation } \\
\left(\delta\left(r^{*}\right) / \delta(0)\right)\end{array}$ & $v^{*}\left(r^{*}\right)$ \\
\hline 1 & 1 & 0 & 1 \\
\hline 0.8377 & 0.8520 & 0.01429 & 1.0588 \\
\hline 0.5656 & 0.5831 & 0.01753 & 0.9666 \\
\hline 0.3169 & 0.3279 & 0.01099 & 1.0574 \\
\hline 0.1539 & 0.1601 & 0.006173 & 0.9683 \\
\hline 0.06818 & 0.07103 & 0.002852 & 0.8689 \\
\hline 0.02325 & 0.02672 & 0.003472 & 0.5171 \\
\hline 0.003097 & 0.005816 & 0.002719 & 0.3204 \\
\hline
\end{tabular}

Table B-6: Measured $\delta\left(r^{*}\right) / \delta(0)$ (erosion time $=300$ s) and calculated $v^{*}\left(r^{*}\right)$ vs. $r^{*}$ (corresponding to Figure 3.9 of Section 3.3, analysis of Section 4.2, and Figure 4.10 of Section 4.3) using $k=1.6$ and $k=2.7$ for $50 \mu \mathrm{m}$ silica glass beads blasted at $d=10 \mathrm{~mm}$ at a pressure of $200 \mathrm{kPa}$ and a flow rate of $0.23 \mathrm{~g} / \mathrm{min}$ using $0.46 \mathrm{~mm}$ H.P. round nozzle.

\begin{tabular}{|c|c|c|c|c|c|}
\hline$r(\mathrm{~mm})$ & $r^{*}$ & $\begin{array}{c}\text { Avg. } \\
M\left(R^{*}\right) / M(0)\end{array}$ & $\delta(r)(\mathrm{mm})$ & $\delta\left(r^{*}\right) / \delta(0)$ & $\begin{array}{c}\delta(r)(\mathrm{mm}) \\
(\text { repeat })\end{array}$ \\
\hline 0 & 0 & 1 & -0.045 & 1 & -0.0716 \\
\hline 0.3 & 0.03 & 1.1414 & -0.0357 & 0.7933 & -0.0617 \\
\hline 0.6 & 0.06 & 0.9643 & -0.0194 & 0.4311 & -0.0386 \\
\hline 0.9 & 0.09 & 0.6376 & -0.00677 & 0.1504 & -0.0143 \\
\hline 1.2 & 0.12 & 0.2934 & -0.00238 & 0.05289 & -0.00563 \\
\hline 1.5 & 0.15 & 0.1819 & -0.000327 & 0.007267 & -0.0006 \\
\hline 1.8 & 0.18 & 0.1228 & -0.000263 & 0.005844 & -0.000301 \\
\hline 2.1 & 0.21 & 0.08043 & -0.000161 & 0.003578 & -0.000274 \\
\hline
\end{tabular}

Table B-6...Continued.

\begin{tabular}{|c|c|c|c|c|}
\hline $\begin{array}{c}\delta\left(r^{*}\right) / \delta(0) \\
(\text { repeat })\end{array}$ & $\begin{array}{c}\text { Avg. } \\
\delta\left(r^{*}\right) / \delta(0)\end{array}$ & $\begin{array}{c}\text { Standard } \\
\text { Deviation } \\
\left(\delta\left(r^{*}\right) / \delta(0)\right)\end{array}$ & $\begin{array}{c}v^{*}\left(r^{*}\right) \\
(k=1.6)\end{array}$ & $\begin{array}{c}v^{*}\left(r^{*}\right) \\
(k=2.7)\end{array}$ \\
\hline 1 & 1 & 0 & 1 & 1 \\
\hline 0.8617 & 0.8275 & 0.03420 & 0.818 & 0.8877 \\
\hline 0.5391 & 0.4851 & 0.054 & 0.6509 & 0.7754 \\
\hline 0.1997 & 0.1751 & 0.02464 & 0.4458 & 0.6196 \\
\hline 0.07863 & 0.06576 & 0.01287 & 0.3927 & 0.5747 \\
\hline 0.008380 & 0.007823 & 0.0005566 & 0.14 & 0.3118 \\
\hline 0.004204 & 0.005024 & 0.0008203 & 0.1356 & 0.3061 \\
\hline 0.003827 & 0.003702 & 0.0001245 & 0.146 & 0.3198 \\
\hline
\end{tabular}


Table B-7: Measured $\delta\left(r^{*}\right) / \delta(0)$ (erosion time $=10$ s) and calculated $v^{*}\left(r^{*}\right)$ vs. $r^{*}$ (corresponding to Figure 3.10 of Section 3.3, analysis of Section 4.2, and Figure 4.11 of Section 4.3) using $k=1.6$ and $k=2.7$ for $50 \mu \mathrm{m}$ silica glass beads blasted at $d=20 \mathrm{~mm}$ at a pressure of $200 \mathrm{kPa}$ and a flow rate of $1.31 \mathrm{~g} / \mathrm{min}$ using $1.5 \mathrm{~mm}$ H.P. round nozzle.

\begin{tabular}{|c|c|c|c|c|c|}
\hline$r(\mathrm{~mm})$ & $r^{*}$ & $\begin{array}{c}\text { Avg. } \\
\left(R^{*}\right) / M(0)\end{array}$ & $\delta(r)(\mathrm{mm})$ & $\delta\left(r^{*}\right) / \delta(0)$ & $\begin{array}{c}\delta(r)(\mathrm{mm}) \\
(\text { repeat })\end{array}$ \\
\hline 0 & 0 & 1 & -0.0357 & 1 & -0.0273 \\
\hline 0.3 & 0.015 & 0.8772 & -0.0327 & 0.9160 & -0.0249 \\
\hline 0.6 & 0.03 & 0.7343 & -0.0247 & 0.6919 & -0.0165 \\
\hline 1 & 0.05 & 0.3794 & -0.0157 & 0.4398 & -0.01 \\
\hline 1.5 & 0.075 & 0.1991 & -0.00711 & 0.1992 & -0.00311 \\
\hline 2 & 0.1 & 0.06955 & -0.00283 & 0.07927 & -0.0015 \\
\hline 2.5 & 0.125 & 0.06159 & -0.000745 & 0.02087 & -0.000369 \\
\hline 3 & 0.15 & 0.02488 & -0.000133 & 0.003725 & -0.0000442 \\
\hline
\end{tabular}

Table B-7...Continued.

\begin{tabular}{|c|c|c|c|c|}
\hline $\begin{array}{c}\delta\left(r^{*}\right) / \delta(0) \\
\text { (repeat) }\end{array}$ & $\begin{array}{c}\text { Avg. } \\
\delta\left(r^{*}\right) / \delta(0)\end{array}$ & $\begin{array}{c}\text { Standard } \\
\text { Deviation } \\
\left(\delta\left(r^{*}\right) / \delta(0)\right)\end{array}$ & $\begin{array}{c}v^{*}\left(r^{*}\right) \\
(k=1.6)\end{array}$ & $\begin{array}{c}v^{*}\left(r^{*}\right) \\
(k=2.7)\end{array}$ \\
\hline 1 & 1 & 0 & 1 & 1 \\
\hline 0.9121 & 0.9140 & 0.001939 & 1.026 & 1.0154 \\
\hline 0.6044 & 0.6481 & 0.04374 & 0.925 & 0.9548 \\
\hline 0.3663 & 0.4030 & 0.03674 & 1.0385 & 1.0226 \\
\hline 0.1139 & 0.1565 & 0.04262 & 0.8605 & 0.9148 \\
\hline 0.05495 & 0.06711 & 0.01216 & 0.9779 & 0.9868 \\
\hline 0.01352 & 0.01719 & 0.003676 & 0.4504 & 0.6233 \\
\hline 0.001619 & 0.002672 & 0.001053 & 0.248 & 0.4376 \\
\hline
\end{tabular}

Table B-8a: Measured $\delta\left(r^{*}\right) / \delta(0)$ (erosion time $=5$ s) and calculated $v^{*}\left(r^{*}\right)$ vs. $r^{*}$ (corresponding to Figure 3.11 of Section 3.3, analysis of Section 4.2, and Figure 4.12 of Section 4.3) using $k=1.44$ (Figure 4.3) for $10 \mu \mathrm{m}$ angular aluminum oxide blasted at $d=30$ $\mathrm{mm}$ at a pressure of $300 \mathrm{kPa}$ and a flow rate of $4.20 \mathrm{~g} / \mathrm{min}$ using $0.76 \mathrm{~mm}$ round nozzle.

\begin{tabular}{|c|c|c|c|c|c|}
\hline$r(\mathrm{~mm})$ & $r^{*}$ & $\begin{array}{c}\text { Avg. } \\
M\left(R^{*}\right) / M(0)\end{array}$ & $\delta(r)(\mathrm{mm})$ & $\delta\left(r^{*}\right) / \delta(0)$ & $\begin{array}{c}\delta(r)(\mathrm{mm}) \\
(\text { repeat })\end{array}$ \\
\hline 0 & 0 & 1 & -0.117 & 1 & -0.133 \\
\hline 0.6 & 0.02 & 1.1143 & -0.0882 & 0.7538 & -0.0946 \\
\hline 1.2 & 0.04 & 0.7524 & -0.0404 & 0.3453 & -0.0412 \\
\hline 1.8 & 0.06 & 0.2109 & -0.0137 & 0.1171 & -0.0135 \\
\hline 2.4 & 0.08 & 0.09264 & -0.0036 & 0.03077 & -0.00365 \\
\hline 3 & 0.1 & 0.04577 & -0.000667 & 0.0057 & -0.000898 \\
\hline 3.6 & 0.12 & 0.03044 & -0.0000174 & 0.0001487 & -0.00000862 \\
\hline 4.2 & 0.14 & 0.01060 & -0.0000416 & 0.0003556 & -0.0000275 \\
\hline
\end{tabular}


Table B-8a...Continued.

\begin{tabular}{|c|c|c|c|}
\hline $\begin{array}{c}\delta\left(r^{*}\right) / \delta(0) \\
(\text { repeat })\end{array}$ & $\begin{array}{c}\text { Avg. } \\
\delta\left(r^{*}\right) / \delta(0)\end{array}$ & $\begin{array}{c}\text { Standard } \\
\text { Deviation } \\
\left(\delta\left(r^{*}\right) / \delta(0)\right)\end{array}$ & $v^{*}\left(r^{*}\right)$ \\
\hline 1 & 1 & 0 & 1 \\
\hline 0.7113 & 0.7326 & 0.02128 & 0.7473 \\
\hline 0.3098 & 0.3275 & 0.01776 & 0.5613 \\
\hline 0.1015 & 0.1093 & 0.007795 & 0.6335 \\
\hline 0.02744 & 0.02911 & 0.001663 & 0.4475 \\
\hline 0.006752 & 0.006226 & 0.0005255 & 0.2502 \\
\hline 0.00006481 & 0.0001068 & 0.00004195 & 0.01973 \\
\hline 0.0002068 & 0.0002811 & 0.0000744 & 0.08041 \\
\hline
\end{tabular}

Table B-8b: Measured normalized average erosion rate, $E^{*}$, as a function of $\sin (\alpha)$ (corresponding to Figure 4.3 and Table B-8a). Best fit velocity exponent $k=1.44$. Erosion of Borofloat glass by $10 \mu \mathrm{m}$ aluminum oxide particles blasted at $d=30 \mathrm{~mm}$ for $60 \mathrm{~s}$ at a pressure of $300 \mathrm{kPa}$ and a flow rate of $4.20 \mathrm{~g} / \mathrm{min}$ using $0.76 \mathrm{~mm}$ round nozzle.

\begin{tabular}{|c|c|c|c|c|c|}
\hline$\alpha$ (deg.) & $\sin (\alpha)$ & $\begin{array}{c}\text { Mass Loss of } \\
\text { Substrate/min } \\
(\mathrm{g} / \mathrm{min})\end{array}$ & $\begin{array}{c}\text { Mass Loss of } \\
\text { Substrate/min } \\
(\mathrm{g} / \mathrm{min}) \\
\text { (repeat) }\end{array}$ & $\begin{array}{c}\text { Standard } \\
\text { Deviation } \\
\text { (Mass loss of } \\
\text { Substrate/min) } \\
(\mathrm{g} / \mathrm{min})\end{array}$ & $\begin{array}{c}\text { Avg. Mass } \\
\text { Loss of } \\
\text { Substrate/min }) \\
(\mathrm{g} / \mathrm{min})\end{array}$ \\
\hline 90 & 1 & 0.0122 & 0.014 & 0.0009 & 0.0131 \\
\hline 72.5 & 0.9537 & 0.0126 & 0.012 & 0.0003 & 0.0123 \\
\hline 60 & 0.8660 & 0.011 & 0.0109 & 0.00005 & 0.01095 \\
\hline 45 & 0.7071 & 0.0065 & 0.0081 & 0.0008 & 0.0073 \\
\hline 30 & 0.5 & 0.004 & 0.0059 & 0.00095 & 0.00495 \\
\hline
\end{tabular}

Table B-8b...Continued.

\begin{tabular}{|c|c|}
\hline $\begin{array}{c}E \text { (using flow } \\
\text { rate from } \\
\text { Figure 3.11) }\end{array}$ & $\begin{array}{c}E^{*} \\
\text { (normalized } \\
\text { with respect } \\
\text { to } E \text { at } \alpha=90^{\circ} \text { ) }\end{array}$ \\
\hline 0.003119 & 1 \\
\hline 0.002929 & 0.9389 \\
\hline 0.002607 & 0.8359 \\
\hline 0.001738 & 0.5573 \\
\hline 0.001179 & 0.3779 \\
\hline
\end{tabular}


Table B-9a: Measured $\delta\left(r^{*}\right) / \delta(0)$ (erosion time $=3$ s) and calculated $v^{*}\left(r^{*}\right)$ vs. $r^{*}$ (corresponding to Figure 3.12 of Section 3.3, analysis of Section 4.2, and Figure 4.13 of Section 4.3) using $k=1.59$ (Figure 4.4) for $25 \mu \mathrm{m}$ angular aluminum oxide blasted at $d=30$ $\mathrm{mm}$ at a pressure of $300 \mathrm{kPa}$ and a flow rate of $3.10 \mathrm{~g} / \mathrm{min}$ using $0.76 \mathrm{~mm}$ round nozzle.

\begin{tabular}{|c|c|c|c|c|c|}
\hline$r(\mathrm{~mm})$ & $r^{*}$ & $\begin{array}{c}\text { Avg. } \\
M\left(R^{*}\right) / M(0)\end{array}$ & $\delta(r)(\mathrm{mm})$ & $\delta\left(r^{*}\right) / \delta(0)$ & $\begin{array}{c}\delta(r)(\mathrm{mm}) \\
(\text { repeat })\end{array}$ \\
\hline 0 & 0 & 1 & -0.134 & 1 & -0.128 \\
\hline 0.3 & 0.01 & 0.9187 & -0.127 & 0.9478 & -0.12 \\
\hline 0.6 & 0.02 & 0.8915 & -0.109 & 0.8134 & -0.109 \\
\hline 1 & 0.0333 & 0.7354 & -0.0786 & 0.5866 & -0.0804 \\
\hline 1.5 & 0.05 & 0.5765 & -0.0452 & 0.3373 & -0.0484 \\
\hline 2 & 0.0667 & 0.3390 & -0.0224 & 0.1672 & -0.0241 \\
\hline 2.5 & 0.0833 & 0.1753 & -0.00908 & 0.06776 & -0.0102 \\
\hline 3 & 0.1 & 0.09991 & -0.00283 & 0.02112 & -0.00429 \\
\hline
\end{tabular}

Table B-9a...Continued.

\begin{tabular}{|c|c|c|c|}
\hline $\begin{array}{c}\delta\left(r^{*}\right) / \delta(0) \\
\text { (repeat) }\end{array}$ & $\begin{array}{c}\text { Avg. } \\
\delta\left(r^{*}\right) / \delta(0)\end{array}$ & $\begin{array}{c}\text { Standard } \\
\text { Deviation } \\
\left(\delta\left(r^{*}\right) / \delta(0)\right)\end{array}$ & $v^{*}\left(r^{*}\right)$ \\
\hline 1 & 1 & 0 & 1 \\
\hline 0.9375 & 0.9426 & 0.005131 & 1.0163 \\
\hline 0.8516 & 0.8325 & 0.01907 & 0.9579 \\
\hline 0.6281 & 0.6073 & 0.02078 & 0.8867 \\
\hline 0.3781 & 0.3577 & 0.02041 & 0.7407 \\
\hline 0.1883 & 0.1777 & 0.01056 & 0.6662 \\
\hline 0.07969 & 0.07372 & 0.005963 & 0.5800 \\
\hline 0.03352 & 0.02732 & 0.006198 & 0.4424 \\
\hline
\end{tabular}

Table B-9b: Measured normalized average erosion rate, $E^{*}$, as a function of $\sin (\alpha)$ (corresponding to Figure 4.4 and Table B-9a). Best fit velocity exponent $k=1.59$. Erosion of Borofloat glass by $25 \mu \mathrm{m}$ aluminum oxide particles blasted at $d=30 \mathrm{~mm}$ for $60 \mathrm{~s}$ at a pressure of $300 \mathrm{kPa}$ and a flow rate of $3.10 \mathrm{~g} / \mathrm{min}$ using $0.76 \mathrm{~mm}$ round nozzle.

\begin{tabular}{|c|c|c|c|c|c|}
\hline$\alpha($ deg.) & $\sin (\alpha)$ & $\begin{array}{c}\text { Mass Loss of } \\
\text { Substrate/min } \\
(\mathrm{g} / \mathrm{min})\end{array}$ & $\begin{array}{c}\text { Mass Loss of } \\
\text { Substrate/min } \\
(\mathrm{g} / \mathrm{min}) \\
\text { (repeat) }\end{array}$ & $\begin{array}{c}\text { Standard } \\
\text { Deviation } \\
(\text { Mass loss of } \\
\text { Substrate/min }) \\
(\mathrm{g} / \mathrm{min})\end{array}$ & $\begin{array}{c}\text { Avg. Mass } \\
\text { Loss of } \\
\text { Substrate/min }) \\
(\mathrm{g} / \mathrm{min})\end{array}$ \\
\hline 90 & 1 & 0.0269 & 0.0247 & 0.0011 & 0.0258 \\
\hline 72.5 & 0.9537 & 0.0237 & 0.0231 & 0.0003 & 0.0234 \\
\hline 60 & 0.8660 & 0.0179 & 0.0186 & 0.00035 & 0.01825 \\
\hline 45 & 0.7071 & 0.0141 & 0.0124 & 0.00085 & 0.01325 \\
\hline 30 & 0.5 & 0.0097 & 0.0072 & 0.00125 & 0.00845 \\
\hline
\end{tabular}


Table B-9b...Continued.

\begin{tabular}{|c|c|}
\hline $\begin{array}{c}E \text { (using flow } \\
\text { rate from } \\
\text { Figure 3.12) }\end{array}$ & $\begin{array}{c}E^{*} \\
\text { (normalized } \\
\text { with respect } \\
\text { to } E \text { at } \alpha=90^{\circ} \text { ) }\end{array}$ \\
\hline 0.008323 & 1 \\
\hline 0.007548 & 0.9070 \\
\hline 0.005887 & 0.7074 \\
\hline 0.004274 & 0.5136 \\
\hline 0.002726 & 0.3275 \\
\hline
\end{tabular}

Table B-10: Measured $\delta\left(r^{*}\right) / \delta(0)$ (erosion time $=150$ s) and calculated $v^{*}\left(r^{*}\right)$ vs. $r^{*}$ (corresponding to Figure 3.13 of Section 3.3, analysis of Section 4.2, and Figure 4.14 of Section 4.3) using $k=1.6$ and $k=2.7$ for $50 \mu \mathrm{m}$ silica glass beads blasted at $d=20 \mathrm{~mm}$ at a pressure of $300 \mathrm{kPa}$ and a flow rate of $1.34 \mathrm{~g} / \mathrm{min}$ using $0.76 \mathrm{~mm}$ round nozzle.

\begin{tabular}{|c|c|c|c|c|c|}
\hline$r(\mathrm{~mm})$ & $r^{*}$ & $\begin{array}{c}\text { Avg. } \\
M\left(R^{*}\right) / M(0)\end{array}$ & $\delta(r)(\mathrm{mm})$ & $\delta\left(r^{*}\right) / \delta(0)$ & $\begin{array}{c}\delta(r)(\mathrm{mm}) \\
\text { (repeat) }\end{array}$ \\
\hline 0 & 0 & 1 & -0.0479 & 1 & -0.111 \\
\hline 0.3 & 0.015 & 1.0516 & -0.0366 & 0.7641 & -0.0952 \\
\hline 0.6 & 0.03 & 0.8793 & -0.0291 & 0.6075 & -0.0667 \\
\hline 1 & 0.05 & 0.6312 & -0.0183 & 0.3820 & -0.0343 \\
\hline 1.5 & 0.075 & 0.4374 & -0.00619 & 0.1292 & -0.011 \\
\hline 2 & 0.1 & 0.2323 & -0.00274 & 0.0572 & -0.00369 \\
\hline 2.5 & 0.125 & 0.1436 & -0.000269 & 0.005616 & -0.000329 \\
\hline 3 & 0.15 & 0.04392 & -0.000068 & 0.00142 & -0.0000621 \\
\hline
\end{tabular}

Table B-10...Continued.

\begin{tabular}{|c|c|c|c|c|}
\hline $\begin{array}{c}\delta\left(r^{*}\right) / \delta(0) \\
(\text { repeat })\end{array}$ & $\begin{array}{c}\text { Avg. } \\
\delta\left(r^{*}\right) / \delta(0)\end{array}$ & $\begin{array}{c}\text { Standard } \\
\text { Deviation } \\
\left(\delta\left(r^{*}\right) / \delta(0)\right)\end{array}$ & $\begin{array}{c}v^{*}\left(r^{*}\right) \\
(k=1.6)\end{array}$ & $\begin{array}{c}v^{*}\left(r^{*}\right) \\
(k=2.7)\end{array}$ \\
\hline 1 & 1 & 0 & 1 & 1 \\
\hline 0.8577 & 0.8109 & 0.04678 & 0.85 & 0.9082 \\
\hline 0.6009 & 0.6042 & 0.003307 & 0.791 & 0.8703 \\
\hline 0.309 & 0.3455 & 0.03652 & 0.6862 & 0.8 \\
\hline 0.0991 & 0.1142 & 0.01506 & 0.4319 & 0.608 \\
\hline 0.03324 & 0.04522 & 0.01198 & 0.3596 & 0.5455 \\
\hline 0.002964 & 0.00429 & 0.001326 & 0.1114 & 0.2725 \\
\hline 0.0005595 & 0.0009895 & 0.0004301 & 0.09343 & 0.2454 \\
\hline
\end{tabular}


Table B-11: Measured $\delta\left(r^{*}\right) / \delta(0)$ (erosion time $=240$ s) and calculated $v^{*}\left(r^{*}\right)$ vs. $r^{*}$ (corresponding to Figure 3.6 of Section 3.3, analysis of Section 4.2, and Figure 4.15 of Section 4.3) using $k=1.6$ and $k=2.7$ for $50 \mu \mathrm{m}$ silica glass beads blasted at $d=20 \mathrm{~mm}$ at a pressure of $200 \mathrm{kPa}$ and a flow rate of $0.59 \mathrm{~g} / \mathrm{min}$ using $0.76 \mathrm{~mm}$ round nozzle.

\begin{tabular}{|c|c|c|c|c|c|}
\hline$r(\mathrm{~mm})$ & $r^{*}$ & $\begin{array}{c}\text { Avg. } \\
M\left(R^{*}\right) / M(0)\end{array}$ & $\delta(r)(\mathrm{mm})$ & $\delta\left(r^{*}\right) / \delta(0)$ & $\begin{array}{c}\delta(r)(\mathrm{mm}) \\
(\text { repeat })\end{array}$ \\
\hline 0 & 0 & 1 & -0.0438 & 1 & -0.0222 \\
\hline 0.3 & 0.015 & 0.8675 & -0.0335 & 0.7648 & -0.0179 \\
\hline 0.6 & 0.03 & 0.7632 & -0.0207 & 0.4726 & -0.012 \\
\hline 1 & 0.05 & 0.4226 & -0.00993 & 0.2267 & -0.00565 \\
\hline 1.5 & 0.075 & 0.2908 & -0.00475 & 0.1084 & -0.00227 \\
\hline 2 & 0.1 & 0.1476 & -0.00111 & 0.02534 & -0.000586 \\
\hline 2.5 & 0.125 & 0.07416 & -0.000467 & 0.01066 & -0.00012 \\
\hline 3 & 0.15 & 0.02378 & -0.0000475 & 0.001084 & -0.00000653 \\
\hline
\end{tabular}

Table B-11...Continued.

\begin{tabular}{|c|c|c|c|c|}
\hline $\begin{array}{c}\delta\left(r^{*}\right) / \delta(0) \\
(\text { repeat })\end{array}$ & $\begin{array}{c}\text { Avg. } \\
\delta\left(r^{*}\right) / \delta(0)\end{array}$ & $\begin{array}{c}\text { Standard } \\
\text { Deviation } \\
\left(\delta\left(r^{*}\right) / \delta(0)\right)\end{array}$ & $\begin{array}{c}v^{*}\left(r^{*}\right) \\
(k=1.6)\end{array}$ & $\begin{array}{c}v^{*}\left(r^{*}\right) \\
(k=2.7)\end{array}$ \\
\hline 1 & 1 & 0 & 1 & 1 \\
\hline 0.8063 & 0.7856 & 0.02073 & 0.9399 & 0.964 \\
\hline 0.5405 & 0.5066 & 0.03397 & 0.774 & 0.8592 \\
\hline 0.2545 & 0.2406 & 0.0139 & 0.7033 & 0.8118 \\
\hline 0.1023 & 0.1053 & 0.003098 & 0.5302 & 0.6866 \\
\hline 0.0264 & 0.02587 & 0.000527 & 0.3368 & 0.5247 \\
\hline 0.005405 & 0.008034 & 0.002628 & 0.2493 & 0.439 \\
\hline 0.0002941 & 0.0006893 & 0.0003952 & 0.1094 & 0.2694 \\
\hline
\end{tabular}




\section{References}

[1] P.H. Shipway and I.M. Hutchings, A method for optimizing the particle flux in erosion testing with a gas-blast apparatus, Wear 174 (1994) 169-175.

[2] P. Chevallier and A.B. Vannes, Effects on a sheet surface of an erosive particle jet upon impact, Wear 184 (1995) 87-91.

[3] F.H. in't Veld and P.J. Slikkerveer, Towards prediction of flux effects in powder blasting nozzles, Wear 215 (1998) 131-136.

[4] Y.I. Oka, M. Nishimura, K. Nagahashi and M. Matsumura, Control and evaluation of particle impact conditions in a sand erosion test facility, Wear 250 (2001) 736-743.

[5] M. Liebhard, and A. Levy, The effect of erodent particle characteristics on the erosion of metals, Wear 151 (1991) 381-390.

[6] P.R. Krishnamoorthy, S. Seetharamu and P. Sampathkumaran, Influence of the mass flux and impact angle of the abrasive on the erosion resistance of materials used in pulverized fuel bends and other components in thermal power stations, Wear 165 (1993) 151-157.

[7] Y.I. Oka, H. Ohnogi, T. Hosokawa and M. Matsumura, The impact angle dependence of erosion damage caused by solid particle impact, Wear 203-204 (1997) 573-579.

[8] D.R. Andrews and N. Horsfield, Particle collisions in the vicinity of an eroding surface. J. Phys. D: Appl. Phys., 16 (1983) 525-538.

[9] K. Anand, S.K. Hovis, H. Conrad and R.O. Scattergood, Flux effects in solid particle erosion, Wear 118 (1987) 243-257.

[10] D. Ciampini, J.K. Spelt and M. Papini, Simulation of interference effects in particle streams following impact with a flat surface, Part I: Theory and analysis, Wear 254 (2003) 237-249.

[11] R. M. Brach, Impact dynamics with applications to solid particle erosion, Int. J. Impact Engng., 7, No.1 (1988) 37-53.

[12] D. Ciampini, J.K. Spelt and M. Papini, Simulation of interference effects in particle streams following impact with a flat surface, Part II: Parametric study and implications for erosion testing and blast cleaning, Wear 254 (2003) 250-264.

[13] M. Papini, D. Ciampini, T. Krajac and J.K. Spelt, Computer modeling of interference effects in erosion testing: effect of plume shape, Wear 255 (2003) 85-97.

[14] C. Gomes-Ferreira, D. Ciampini and M. Papini, The effect of inter-particle collisions in erosive streams on the distribution of energy incident to a flat surface, Tribology International, 37 (2004) 791-807.

[15] P.H. Shipway and I.M. Hutchings, Influence of nozzle roughness on conditions in a gasblast erosion rig, Wear, 162-164 (1993) 148-158.

[16] A.N.J. Stevenson and I.M. Hutchings, The influence of nozzle length on the divergence of the erodent particle stream in a gas-blast erosion rig, Wear, 189 (1995) 66-69.

[17] P.H. Shipway, The effect of plume divergence on the spatial distribution and magnitude of wear in gas-blast erosion, Wear, 205 (1997) 169-177.

[18] R. Balasubramaniam, J. Krishnan and N. Ramakrishnan, A study on the shape of surface generated by abrasive jet machining, Journal of Materials Processing Technology, 121 (2002) 102-106.

[19] M. Achtsnick, P.F. Geelhoed, A.M. Hoogstrate and B. Karpuschewski, Modeling and evaluation of micro abrasive blasting process, Wear, 259 (2005) 84-94. 
[20] P.J. Slikkerveer and F.H. in't Veld, Model for patterned erosion, Wear, 233-235 (1999) 377-386.

[21] J.H.M. Ten Thije Boonkkamp and J.K.M. Jansen, An analytical solution for mechanical etching of glass by powder blasting, Journal of Engineering Mathematics, 43 (2002) 385-399.

[22] P.J. Slikkerveer, P.C.P. Bouten, F.H. in't Veld and H. Scholten, Erosion and damage by sharp particles, Wear 217 (1998) 237-250.

[23] Y. Ballout, J.A. Mathis and J.E. Talia, Solid particle erosion mechanisms in glass, Wear 196 (1996) 263-269.

[24] T. Burzynski and M. Papini, Analytical models of the interference between incident and rebounding particles within an abrasive jet: Comparison with computer simulation, Wear (WOM 2007 Special Issue), accepted, December, 2006.

[25] A. Ghobeity, T. Krajac, T. Burzynski, M. Papini and J.K. Spelt, Surface evolution models in abrasive jet micromachining, Wear, accepted, February, 2007.

[26] T. Burzynski and M. Papini, A Novel Approach to Measure the Particle Distribution within a Particle Laden Jet, Proceedings of the CANCAM Forum (CD ROM), Toronto, Ontario, Canada, June 3-7, 2007.

[27] T. Burzynski, Design of a Device to Obtain a Particle Probability Distribution, Undergraduate Thesis, Department of Industrial and Mechanical Engineering, Ryerson University, Toronto, Canada (2005).

[28] J.T. Davies, Turbulence Phenomena, Academic Press (1972) 66.

[29] Z. Feng and A. Ball, The erosion of four materials using seven erodents - towards an understanding, Wear 233-235 (1999) 674-684.

[30] H. Uuemois and I. Kleis, A critical analysis of erosion problems which have been little studied, Wear 31 (1975) 359-371.

[31] W.D. Bachalo and M.J. Houser, Phase Doppler spray analyzer for simultaneous measurements of drop size and velocity distributions, Optical Eng. 23 (1984) 583-590.

[32] A.P. Verma and G.K. Lai, A theoretical study of erosion phenomenon in abrasive jet machining, Journal of Manufacturing Science and Engineering 118 (1996) 564-570.

[33] A.A. Mostafa, V.G. McDonell, H.C. Mongia and G.S. Samuelsen, An experimental and numerical study of particle-laden coaxial jet flows, Int. J. Heat and Fluid Flow, 11, No. 2
(1990) 90-97.

[34] Y. Hardalupas, A.M.K.P. Taylor and J.H Whitelaw, Velocity and particle-flux characteristics of turbulent particle-laden jets, Proceedings of the Royal Society of London, Series A, Mathematical and Physical Sciences, 426, No. 1870 (1989) 31-78. 